\title{
Pathways of toxicity after inhalation of nanoparticles
}

The influence of physicochemical properties on the biodistribution, molecular pathways and toxicity

\section{Susan Dekkers}





\section{Pathways of toxicity after inhalation of nanoparticles}

The influence of physicochemical properties on the biodistribution, molecular pathways and toxicity. 
(C) 2021 Susan Dekkers

Thesis Utrecht University

ISBN: 978-90-393-7364-4

Layout by: Dennis Hendriks / ProefschriftMaken.nl

Cover design by: Susan Dekkers

Print: ProefschriftMaken, The Netherlands 


\title{
Pathways of toxicity after inhalation of nanoparticles
}

\author{
The influence of physicochemical properties on the \\ biodistribution, molecular pathways and toxicity.
}

\section{Toxicologische werkingsmechanismen van nanodeeltjes na inhalatie De invloed van fysisch-chemische eigenschappen op de biodistributie, moleculaire mechanismen en toxiciteit (met een samenvatting in het Nederlands)}

\author{
Proefschrift \\ ter verkrijging van de graad van doctor aan de \\ Universiteit Utrecht \\ op gezag van de \\ rector magnificus, prof.dr. H.R.B.M. Kummeling, \\ ingevolge het besluit van het college voor promoties \\ in het openbaar te verdedigen op \\ donderdag 18 maart 2021 des middags te 12.45 uur
}

door

\section{Susan Dekkers}

geboren op 5 mei 1975

te Utrecht 


\section{Promotoren:}

Prof. dr. F.R. Cassee

Prof. dr. ir. B. Brunekreef

\section{Copromotoren:}

Dr. R.J. Vandebriel

Dr. W.H. de Jong

This research was financially supported by the European Union's Seventh Framework Programme for research, technology development and demonstration under grant agreement $n^{\circ} 310451$ (NanoMILE) and the Netherlands Food and Consumer Product Safety Authority (V090016) (NVWA). 


\section{Contents}

$\begin{array}{lll}\text { Chapter } 1 \text { General introduction } & 7\end{array}$

Chapter 2 The effect of zirconium doping of cerium dioxide nanoparticles on $\quad 19$ pulmonary and cardiovascular toxicity and biodistribution in mice after inhalation (Dekkers et al., 2017)

Chapter 3 Differences in the toxicity of cerium dioxide nanomaterials after 57 inhalation can be explained by lung deposition, animal species and nanoforms (Dekkers et al., 2018a)

Chapter 4 Multi-omics approaches confirm metal ions mediate the main toxicological pathways of metal-bearing nanoparticles in lung epithelial A549 cells (Dekkers et al., 2018b)

Chapter 5 Role of chemical composition and redox modification of poorly soluble nanomaterials on their ability to enhance allergic airway sensitisation in mice (Dekkers et al., 2019)

Chapter 6 The influence of chemical composition and redox activity of nanomaterials on in vitro inflammasome activation and dendritic cell maturation

Chapter 7 General discussion

Summary

Samenvatting

Curriculum Vitae 


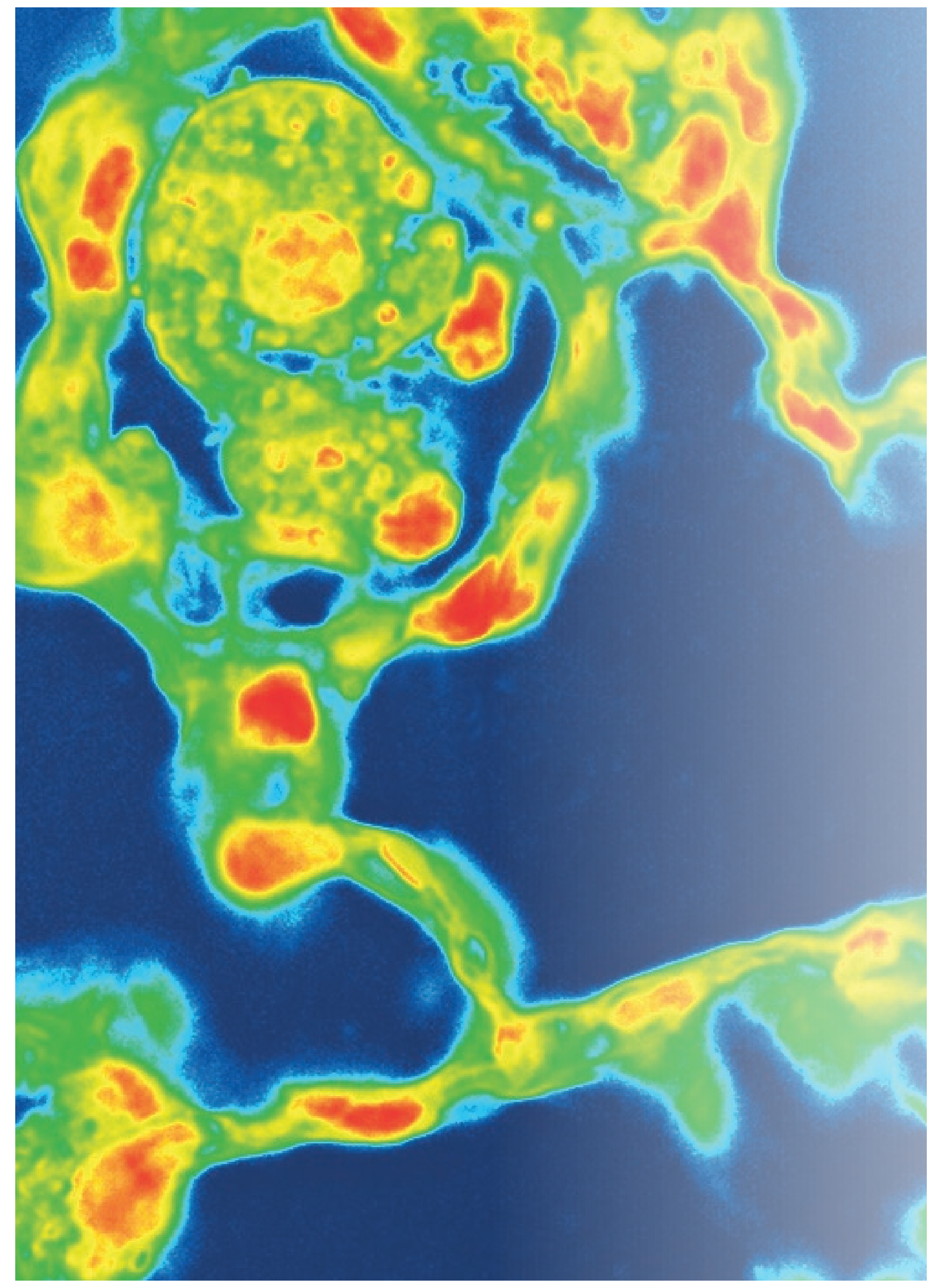


Chapter 1

General Introduction 


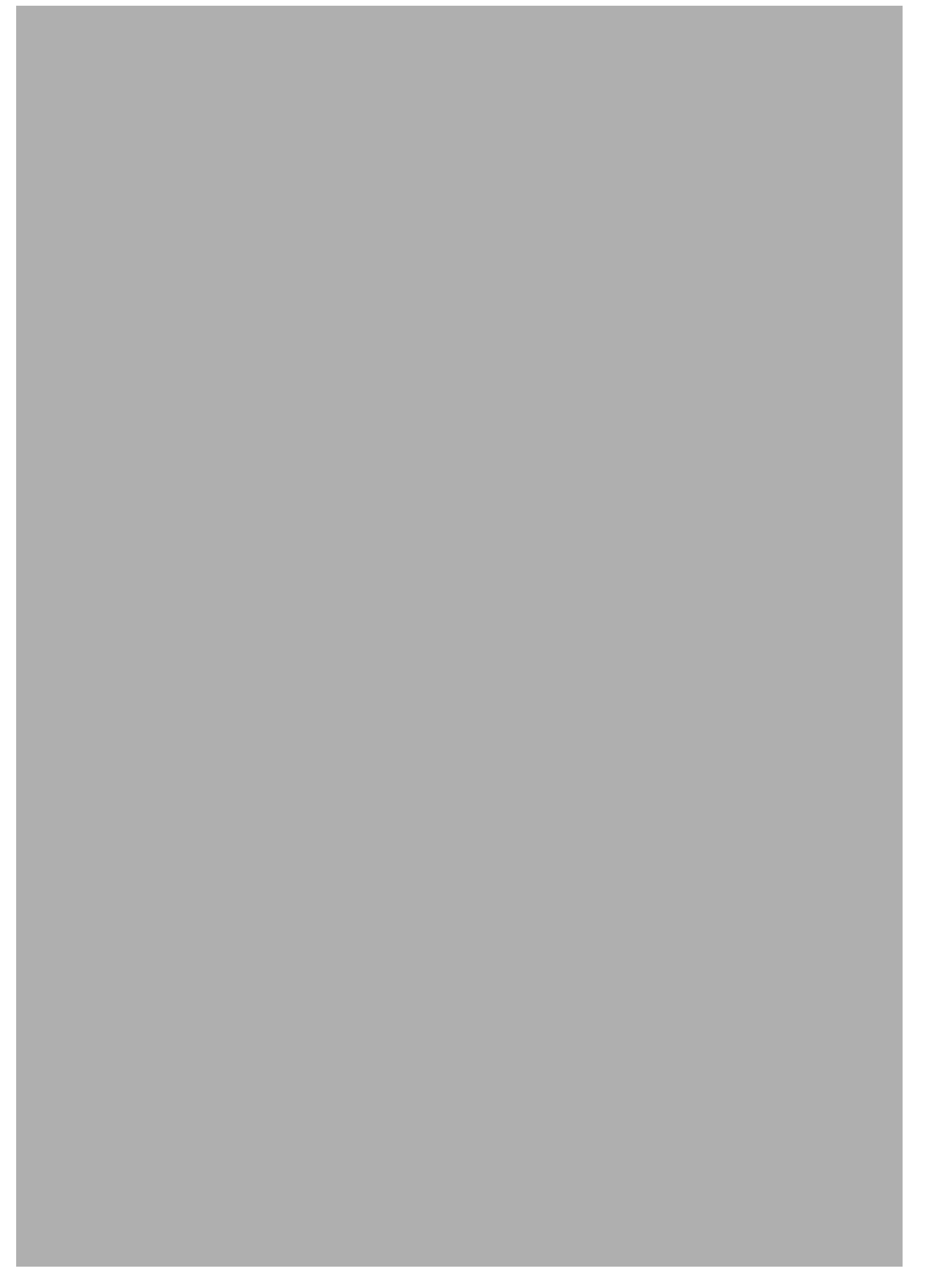




\section{General Introduction}

\section{The influence of physicochemical properties of nanomaterials}

The most appealing feature of nanomaterials is that their properties can be manipulated in such a way they can exhibit the most amazing functionalities. At the same time, this is also the most alarming aspect of nanomaterials, since we don't know exactly yet how manipulating their properties might affect their ability to cause adverse human health effects. Knowing how the different properties of nanomaterials influence their ability to cause human health effects may enable us to design nanomaterials with amazing functionalities without increasing human health risks.

The aim of the research described in this thesis is to investigate how several physicochemical properties of nanoparticles (NPs) influence their biodistribution, molecular pathways and toxicity after inhalation. The focus is on inhalation, as this route of exposure is currently regarded as the main route of concern for human health effects (Kuhlbusch et al., 2018). In this thesis the results of several in vivo and in vitro toxicity studies on the influence of several physicochemical properties on various biological effects are described. The studies examined the following physicochemical properties: redox activity, dissolution, chemical composition and size. The investigated biological effects included in vivo lung deposition, biodistribution and inflammation, in vitro multi-omics responses in alveolar epithelial cells, in vivo adjuvant activity, in vitro inflammasome activation and in vitro dendritic cell maturation.

\section{Towards predictive toxicology of nanoparticles}

That particles may cause severe human health effects after inhalation has been known for centuries. The history and current knowledge on the toxicity of inhaled particles has been reviewed in several publications (Donaldson and Seaton, 2012; Riediker et al., 2019). Adverse health effects of inhaled particles were first observed among workers exposed to asbestos, crystalline silica and coal dust. The scientific attention for the toxicology of inhaled particles further increased when adverse health effects were also observed in the general population that were associated with poor air quality such as during the 'great smog' episode of 1952 in London (Bell et al., 2004). The publication of the report of the Royal Academy of Engineering and the Royal Society on the opportunities and uncertainties of nanoscience and nanotechnologies in 2004 (Royal Society and Royal Academy of Engineering, 2004) broadened the scientific attention to also include the potential adverse health effects of manufactured NPs.

Nanotechnology makes it possible to manufacture NPs with an unlimited amount of variations in physicochemical properties, such as size, shape and surface modifications. This endless amount of variations makes it practically impossible to test each individual variation or nanoform of each type of NP (Dekkers et al., 2016). Therefore, knowledge on the relation 
between certain physicochemical properties of NPs and their ability to cause adverse effects becomes increasingly important. This knowledge will improve the ability to predict the toxicity of new nanomaterials without extensive toxicity testing of each individual nanoform. Over the last 15 years a number of papers have been published dedicated to predictive toxicity, grouping, and read-across (Arts et al., 2015; Banares et al., 2017; Singh et al., 2019; Stone et al., 2020; Winkler, 2016). Several reviews have appeared in which various mechanisms of toxicity of (nano)particles and their connection to various physicochemical properties are described (Bakand et al., 2012; Bierkandt et al., 2018; Nel et al., 2006). Some mechanisms of toxicity, such as the release of ions or the ability to induce oxidative stress, are quite generic and relevant for multiple adverse effects and routes of exposure. Other mechanisms of toxicity, such as frustrated phagocytosis after translocation to the pleural cavity, are more specific for one adverse effects and one route of exposure, in this case mesothelioma after inhalation of asbestos-like nanomaterials. In reviews on the toxicity of NPs after inhalation, generally a distinction is made between mechanisms and physicochemical properties that determine the biokinetics, including airway and lung deposition, uptake and translocation to secondary organs and mechanisms and physicochemical properties that determine or influence the biological effects of NPs, such as redox activity and the capacity to generate of reactive oxygen species (ROS), the release of ions, cationic toxicity due to mitochondrial perturbation, inflammasome activation, photoactivation, protein and DNA damage, altered cell cycle regulation, phagocytosis impairment, endothelial dysfunction and adjuvant effects(Bakand et al., 2012; Bakand et al., 2005; Braakhuis et al., 2014; Nel et al., 2013).

\section{Linking physicochemical properties to mechanisms of toxicity}

A detailed description and explanation on all the different mechanisms and physicochemical properties is beyond the scope of this thesis. The following paragraph provides a brief review of the mechanisms and properties investigated in this thesis. The focus is on two relations between physicochemical properties and mechanisms of toxicity: 1) redox activity and the capacity of generating ROS and 2) dissolution and the release of ions. Another important mechanisms by which NPs can induce toxicity is via the interaction with the immune system. The immune system is prone to react to foreign intrusions, including particles. Some of these reactions are normal immunological responses to facilitate clearance of the NPs from the body and recovery of the homeostasis, while others may lead to undesired adverse effects (Boraschi et al., 2017). Therefore, also several immunological mechanisms, including the adjuvant activity of NPs, inflammasome activation and dendritic cell maturation have been investigated.

\section{Redox activity and the generation of reactive oxygen species}

One of the most investigated and well established mechanisms by which NPs can induce toxicity is via the generation of reactive oxygen species, which may lead to oxidative stress and subsequent cellular injury and pro-inflammatory signaling (Hsieh et al., 2013; Nel et al., 
2013). The ability of NPs to generate ROS is influenced by several physiochemical properties, including their size or rather their relatively large surface area which makes them more reactive, their shape and their surface characteristics, such as the surface charge, surfacetreatment and redox activity (Fu et al., 2014). Also changes of the NPs due to interaction with their surrounding environment, such as aggregation, UV light activation, dissolution and the release of metal ions can affect their ability to generate ROS. Metal oxides, in particular in the nanosized range, are known to facilitate the formation of ROS by depleting electrons from cellular redox species (cellular components able to release electrons) or by serving as catalysts in ROS production through Fenton reactions or Haber-Weiss cycle reactions (Fu et al., 2014). Additionally, NPs can stimulate free radical generation from cellular enzymes. Metal oxides with a conduction band energy (Ec) level that overlaps with the cellular redox potential $(-4.12$ to $-4.84 \mathrm{eV})$ have been shown to have the ability to induce oxygen radicals, oxidative stress, and acute pulmonary inflammation (Zhang et al., 2012). The generation of ROS does not always cause cellular injury, because biological fluids (e.g. epithelial lining fluid) and cells also contain antioxidants and numerous compensatory mechanisms. Only an overproduction of ROS can lead to failure of cells to maintain their normal physiological redox-regulated functions, resulting in oxidative stress, cellular injury and inflammatory responses (Fu et al., 2014).

\section{Dissolution and the release of ions}

Another well-known mechanism of toxicity by which NPs can induce toxicity, is by the release of ions (ion shedding) (Gebel et al., 2014; Nel et al., 2013). The toxicity of for example Ag, $\mathrm{Cu}$ and $\mathrm{ZnO}$ NPs is in part due to a delayed dissolution and subsequent release of ions which often takes place after cellular uptake of the NPs. The similarity and differences of ion versus solid particle toxicity has been investigated in several studies (Cronholm et al., 2013; Liu et al., 2016). However, the different molecular responses within the various pathways leading to toxicity are not yet fully understood. Physicochemical properties that influence NP dissolution and release of ions include chemical composition, size, surface area and surface chemistry. Next to these intrinsic properties, also environmental conditions, such as $\mathrm{pH}$, temperature and the presence of proteins influence the dissolution of NPs (Shi et al., 2017; Sohal et al., 2018).

\section{Adjuvant activity}

Other important mechanisms by which NPs can induce toxicity is via the interaction with the immune system. Particles are known to be able to induce and to enhance lung inflammation and allergic responses after inhalation (Meldrum et al., 2017). Nano-sized silica and titanium dioxide particles have been shown to enhance allergic airways sensitization in mice (Brandenberger et al., 2013; Vandebriel et al., 2018). There is limited understanding of the different physicochemical properties of NPs which influence their adjuvant properties. Due to the variabilities in the models used and the often limited extend of NM characterization 
in the different studies, no reliable comparison could be made between adverse effects of NMs with different characteristics across different studies (Meldrum et al., 2017). Comparisons within studies indicate that NP composition, size, agglomeration size, surface area, shape, solubility, surface charge and surface coating are all potentially important factors determining the immune responses (Meldrum et al., 2017). However to what extent these properties influence which mechanistic pathways remains to be determined.

\section{Inflammasome activation and dendritic cell maturation}

The first types of immune cells that recognize and respond to inhaled NPs are often macrophages and dendritic cells (DCs) (Boraschi et al., 2017). However, the response of macrophages and DCs to NPs is different. Macrophages usually produce inflammatory chemokines and cytokines to recruit neutrophils and monocytes, while DCs usually respond by maturation and migration to the lymph nodes, the production cytokines and initiation of T-cell proliferation and differentiation. One of the mechanisms by which inflammatory responses of macrophages and DCs can be initiated is inflammasome activation.

Inflammasomes are intracellular protein complexes that upon sensing danger signals can initiate inflammatory responses, including the production of IL-1 $\beta$ and IL-18 (Vandebriel et al., 2016). Inflammasomes can respond to a wide range of danger signals, including pathogen-associated molecular patterns (PAMPs), such as lipopolysaccharide, damageassociated molecular patterns (DAMPs), such as extracellular ATP, UV radiation, chemical sensitizers, asbestos and NPs. Secretion of IL-1 $\beta$ and IL-18 leads to an influx of other immune cells, including neutrophils and dendritic cells to the affected tissues to facilitate clearance of nanomaterials (Sun et al., 2013b). The mechanisms of inflammasome activation induced by NPs are not fully understood. Several studies are suggesting that the physicochemical properties of NPs including the ability to generate ROS, aspect ratio, dispersion state, size and surface chemistry could play major roles in NLRP3 inflammasome activation (Sun et al., 2013b; Vandebriel et al., 2016). Inflammasome activation has been suggested to be one of the mechanisms involved in the adjuvant activity of NPs (Meldrum et al., 2017; Sun et al., 2013a; Vandebriel et al., 2016).

DC are cells of the innate immune system that link the innate to the adaptive immunity. Immature DCs can engulf foreign materials, including NPs, but also apoptotic or necrotic cells. DC maturation is a process in which immature DC become active antigen presenting cells by acquire several properties fundamental properties, including antigen processing and presentation, migration and ability to activate antigen-specific naïve T-cells in secondary lymphoid organs (Feray et al., 2020). Depending on the chemical composition, surface chemistry, shape and dose, NPs may promote DCs' capability to induce an IL-12 induced Th1 response, an IL-4 induced Th2 response and/or an IL-6 induced Th17 response (Ihrie and Bonner, 2018; Jia et al., 2018). The DC maturation assay has been suggested as a promising 
in vitro screen predictive for adjuvant activity of NPs in vivo (Feray et al., 2020; Vandebriel et al., 2018).

\section{Aim, objectives and outline of this thesis}

Although there are several mechanisms of toxicity identified by which NMs may cause adverse effects after inhalation, there are still a lot of knowledge gaps on the precise molecular mechanisms and the influence of the various physicochemical properties of NMs on these mechanisms within the different pathways (gaps). In this thesis research on the influence of several physicochemical properties on some mechanisms of toxicity is further described. Combining the knowledge on the influence and interrelationships between the different physicochemical properties and mechanisms of toxicity will enable us to better predict the toxicity of newly developed NPs or NPs for which limited toxicity data is available. This will facilitate implementation of Safe-by-Design and risk assessment of NPs using predictive toxicity, grouping, and read-across.

The research described in this thesis was performed within the European NanoMILE project ${ }^{1}$. The NanoMILE project included investigations on the biological mechanisms and pathways underlying toxic effects of nanomaterials related to their physicochemical properties. The overarching objective of NanoMile was to formulate an intelligent and powerful paradigm for the mode(s) of interaction between manufactured nanomaterials and organisms or the environment to allow the development of a single framework for the categorization of nanomaterial safety and to create a universally applicable framework for nanosafety.

The main aim of the research described in this thesis is to investigate the influence of physicochemical properties of nanomaterials on assumed pathways of toxicity after inhalation. This was done using a carefully selected range of in vivo and in vitro toxicity assays in combination with a carefully selected set NPs with specific properties that were synthesized within this NanoMILE project, translating into the following research objectives:

1. Assess the influence of size and redox activity of undoped and $\mathrm{Zr}$-doped $\mathrm{CeO}_{2}$ NPs on their deposition, biodistribution and pulmonary (and cardiovascular) effects in mice and rats following inhalation. (chapter $2+3$ )

2. Investigate the influence of dissolution and redox activity of $\mathrm{Ag}, \mathrm{ZnO}$ and undoped and $\mathrm{Zr}$-doped $\mathrm{CeO}_{2} \mathrm{NPs}$ on the molecular pathways by which these NPs induce toxicity in A549 lung cell in vitro (chapter 4).

3. Evaluate the influence of chemical composition and redox activity of undoped and Zrdoped $\mathrm{CeO}_{2}$ and undoped and Fe-doped $\mathrm{Co}_{3} \mathrm{O}_{4}$ NPs on their adjuvant properties in an in vivo model for airway allergy (chapter 5 ).

1 Grand agreement number 310451 (NanoMILE) of the European Union Seventh Framework Programme for research, technology development and demonstration. 
4. Study the influence of chemical composition and redox activity of undoped and Zrdoped $\mathrm{CeO}_{2}$ and undoped and Fe-doped $\mathrm{Co}_{3} \mathrm{O}_{4}$ NPs on in vitro inflammasome activation and dendritic cell maturation (chapter 6).

We hypothesized that redox modification through $\mathrm{Zr}$-doping of $\mathrm{CeO}_{2}$ and Fe-doping of $\mathrm{Co}_{3} \mathrm{O}_{4}$ NPs would lead to a decreased in the in vitro and in vivo toxicity and adjuvant activity of the NPs.

More knowledge on the influence of the physicochemical properties of nanomaterials on the pathways of toxicity is needed to predict the toxicity of nanomaterials and to enable safety assessment of new nanomaterials without extensive toxicity testing of each individual nanoform. The influence of various physicochemical properties of several NPs on the biodistribution, molecular pathways and toxicity after inhalation will be discussed in the following chapters. In chapter 2 and 3 of this thesis, the results of several studies on the influence of the size and redox activity on the deposition, biodistribution and toxicity of $\mathrm{CeO}_{2}$ NPs after inhalation are described. Chapter 4 contains the results of a study on the influence of dissolution and redox activity of $\mathrm{Ag}, \mathrm{ZnO}$ and $\mathrm{CeO}_{2} \mathrm{NPs}$ on the molecular mechanisms causing in vitro toxicity in lung epithelial cells. In chapter 5 the influence of the chemical composition and redox modification of $\mathrm{CeO}_{2}$ and $\mathrm{Co}_{3} \mathrm{O}_{4}$ NPs on their ability to enhance allergic airway sensitization in vivo is discussed, while chapter 6 concerns the investigation on ability of the same NPs to induce in vitro inflammasome activation and dendritic cell maturation and compares the in vitro results with the immune responses observed in the in vivo study. A general discussion reflection on the key findings, implications and future perspectives can be found in chapter 7 . 


\section{References}

Arts JH, Hadi M, Irfan MA, Keene AM, Kreiling R, Lyon D, Maier M, Michel K, Petry T, Sauer UG, Warheit D, Wiench K, Wohlleben W and Landsiedel R (2015) A decision-making framework for the grouping and testing of nanomaterials (DF4nanoGrouping). Regul Toxicol Pharmacol 71:S1-27.

Bakand S, Hayes A and Dechsakulthorn F (2012) Nanoparticles: a review of particle toxicology following inhalation exposure. Inhal Toxicol 24:125-135.

Bakand S, Winder C, Khalil C and Hayes A (2005) Toxicity assessment of industrial chemicals and airborne contaminants: transition from in vivo to in vitro test methods: a review. Inhal Toxicol 17.

Banares MA, Haase A, Tran L, Lobaskin V, Oberdorster G, Rallo R, Leszczynski J, Hoet P, Korenstein R, Hardy B and Puzyn T (2017) CompNanoTox2015: novel perspectives from a European conference on computational nanotoxicology on predictive nanotoxicology. Nanotoxicology 11:839-845.

Bell ML, Davis DL and Fletcher T (2004) A retrospective assessment of mortality from the London smog episode of 1952: the role of influenza and pollution. Environ Health Perspect 112:6-8.

Bierkandt FS, Leibrock L, Wagener S, Laux P and Luch A (2018) The impact of nanomaterial characteristics on inhalation toxicity. Toxicol Res (Camb) 7:321-346.

Boraschi D, Italiani P, Palomba R, Decuzzi P, Duschl A, Fadeel B and Moghimi SM (2017) Nanoparticles and innate immunity: new perspectives on host defence. Semin Immunol 34:33-51.

Braakhuis HM, Park MV, Gosens I, De Jong WH and Cassee FR (2014) Physicochemical characteristics of nanomaterials that affect pulmonary inflammation. Part Fibre Toxicol 11:18.

Brandenberger C, Rowley NL, Jackson-Humbles DN, Zhang Q, Bramble LA, Lewandowski RP, Wagner JG, Chen W, Kaplan BL, Kaminski NE, Baker GL, Worden RM and Harkema JR (2013) Engineered silica nanoparticles act as adjuvants to enhance allergic airway disease in mice. Part Fibre Toxicol 10:26.

Cronholm P, Karlsson HL, Hedberg J, Lowe TA, Winnberg L, Elihn K, Wallinder IO and Möller L (2013) Intracellular uptake and toxicity of $\mathrm{Ag}$ and $\mathrm{CuO}$ nanoparticles: a comparison between nanoparticles and their corresponding metal ions. Small 9:970-982.

Dekkers S, Ma-Hock L, Lynch I, Russ M, Miller MR, Schins RPF, Keller J, Romer I, Kuttler K, Strauss V, De Jong WH, Landsiedel R and Cassee FR (2018a) Differences in the toxicity of cerium dioxide nanomaterials after inhalation can be explained by lung deposition, animal species and nanoforms. Inhal Toxicol:1-14.

Dekkers S, Miller MR, Schins RPF, Romer I, Russ M, Vandebriel RJ, Lynch I, Belinga-Desaunay MF, Valsami-Jones E, Connell SP, Smith IP, Duffin R, Boere JAF, Heusinkveld HJ, Albrecht C, de Jong WH and Cassee FR (2017) The effect of zirconium doping of cerium dioxide nanoparticles on pulmonary and cardiovascular toxicity and biodistribution in mice after inhalation. Nanotoxicology 11:794-808.

Dekkers S, Oomen AG, Bleeker EA, Vandebriel RJ, Micheletti C, Cabellos J, Janer G, Fuentes N, Vazquez-Campos S, Borges T, Silva MJ, Prina-Mello A, Movia D, Nesslany F, Ribeiro AR, Leite PE, Groenewold M, Cassee FR, Sips AJ, Dijkzeul A, van Teunenbroek T and Wijnhoven SW (2016) Towards a nanospecific approach for risk assessment. Regul Toxicol Pharmacol 80:46-59. 
Dekkers S, Wagner JG, Vandebriel RJ, Eldridge EA, Tang SVY, Miller MR, Römer I, de Jong WH, Harkema JR and Cassee FR (2019) Role of chemical composition and redox modification of poorly soluble nanomaterials on their ability to enhance allergic airway sensitisation in mice. Particle and Fibre Toxicology 16:39.

Dekkers S, Williams TD, Zhang J, Zhou J, Vandebriel RJ, De La Fonteyne LJJ, Gremmer ER, He S, Guggenheim EJ, Lynch I, Cassee FR, De Jong WH and Viant MR (2018b) Multi-omics approaches confirm metal ions mediate the main toxicological pathways of metal-bearing nanoparticles in lung epithelial A549 cells. Environmental Science: Nano 5:1506-1517.

Donaldson K and Seaton A (2012) A short history of the toxicology of inhaled particles. Part Fibre Toxicol 9:13.

Feray A, Szely N, Guillet E, Hullo M, Legrand FX, Brun E, Pallardy M and Biola-Vidamment A (2020) How to Address the Adjuvant Effects of Nanoparticles on the Immune System. Nanomaterials (Basel) 10.

Fu PP, Xia Q, Hwang HM, Ray PC and Yu H (2014) Mechanisms of nanotoxicity: generation of reactive oxygen species. J Food Drug Anal 22:64-75.

Gebel T, Foth H, Damm G, Freyberger A, Kramer PJ, Lilienblum W, Rohl C, Schupp T, Weiss C, Wollin KM and Hengstler JG (2014) Manufactured nanomaterials: categorization and approaches to hazard assessment. Arch Toxicol 88:2191-2211.

Hsieh SF, Bello D, Schmidt DF, Pal AK, Stella A, Isaacs JA and Rogers EJ (2013) Mapping the biological oxidative damage of engineered nanomaterials. Small 9:1853-1865.

Ihrie MD and Bonner JC (2018) The Toxicology of Engineered Nanomaterials in Asthma. Curr Environ Health Rep 5:100-109.

Jia J, Zhang Y, Xin Y, Jiang C, Yan B and Zhai S (2018) Interactions Between Nanoparticles and Dendritic Cells: From the Perspective of Cancer Immunotherapy. Front Oncol 8:404.

Kuhlbusch TAJ, Wijnhoven SWP and Haase A (2018) Nanomaterial exposures for worker, consumer and the general public. Nanolmpact 10:11-25.

Liu J, Feng X, Wei L, Chen L, Song B and Shao L (2016) The toxicology of ion-shedding zinc oxide nanoparticles. Crit Rev Toxicol 46:348-384.

Meldrum K, Guo C, Marczylo EL, Gant TW, Smith R and Leonard MO (2017) Mechanistic insight into the impact of nanomaterials on asthma and allergic airway disease. Part Fibre Toxicol 14:45.

Nel A, Xia T, Madler L and Li N (2006) Toxic potential of materials at the nanolevel. Science 311:622627.

Nel AE, Xia T, Meng H, Wang X, Lin S, Ji Z and Zhang H (2013) Nanomaterial toxicity testing in the 21st century: use of a predictive toxicological approach and highthroughput screening. Acc Chem Res 46.

Riediker M, Zink D, Kreyling W, Oberdorster G, Elder A, Graham U, Lynch I, Duschl A, Ichihara G, Ichihara S, Kobayashi T, Hisanaga N, Umezawa M, Cheng TJ, Handy R, Gulumian M, Tinkle S and Cassee F (2019) Particle toxicology and health - where are we? Part Fibre Toxicol 16:19. 
Royal Society and Royal Academy of Engineering (2004) Nanoscience and Nanotechnologies: Opportunities and Uncertainties. , in RS policy document, Royal Society and Royal Academy of Engineering, London.

Shi M, de Mesy Bentley KL, Palui G, Mattoussi H, Elder A and Yang H (2017) The roles of surface chemistry, dissolution rate, and delivered dose in the cytotoxicity of copper nanoparticles. Nanoscale 9:4739-4750.

Singh AV, Laux P, Luch A, Sudrik C, Wiehr S, Wild AM, Santomauro G, Bill I and Sitti M (2019) Review of emerging concepts in nanotoxicology: opportunities and challenges for safer nanomaterial design. Toxicol Mech Methods 29:378-387.

Sohal IS, O'Fallon KS, Gaines P, Demokritou P and Bello D (2018) Ingested engineered nanomaterials: state of science in nanotoxicity testing and future research needs. Part Fibre Toxicol 15:29.

Stone V, Gottardo S, Bleeker EAJ, Braakhuis H, Dekkers S, Fernandes T, Haase A, Hunt N, Hristozov D, Jantunen $\mathrm{P}$, Jeliazkova $\mathrm{N}$, Johnston $\mathrm{H}$, Lamon L, Murphy F, Rasmussen $\mathrm{K}$, Rauscher $\mathrm{H}$, Jiménez AS, Svendsen C, Spurgeon D, Vázquez-Campos S, Wohlleben W and Oomen AG (2020) A framework for grouping and read-across of nanomaterials- supporting innovation and risk assessment. Nano Today 35:100941.

Sun B, Ji Z, Liao YP, Wang M, Wang X, Dong J, Chang CH, Li R, Zhang H, Nel AE and Xia T (2013a) Engineering an effective immune adjuvant by designed control of shape and crystallinity of aluminum oxyhydroxide nanoparticles. ACS Nano 7:10834-10849.

Sun B, Wang X, Ji Z, Li R and Xia T (2013b) NLRP3 inflammasome activation induced by engineered nanomaterials. Small 9:1595-1607.

Vandebriel RJ, Dekkers S, de Jong WH and Cassee FR (2016) An Update on NLRP3 Inflammasome Activation by Engineered Nanomaterials. Current Bionanotechnology 2:40-46.

Vandebriel RJ, Vermeulen JP, van Engelen LB, de Jong B, Verhagen LM, de la Fonteyne-Blankestijn LJ, Hoonakker ME and de Jong WH (2018) The crystal structure of titanium dioxide nanoparticles influences immune activity in vitro and in vivo. Part Fibre Toxicol 15:9.

Winkler DA (2016) Recent advances, and unresolved issues, in the application of computational modelling to the prediction of the biological effects of nanomaterials. Toxicology and Applied Pharmacology 299:96-100.

Zhang H, Ji Z, Xia T, Meng H, Low-Kam C, Liu R, Pokhrel S, Lin S, Wang X, Liao YP, Wang M, Li L, Rallo R, Damoiseaux R, Telesca D, Madler L, Cohen Y, Zink JI and Nel AE (2012) Use of metal oxide nanoparticle band gap to develop a predictive paradigm for oxidative stress and acute pulmonary inflammation. ACS Nano 6:4349-4368. 


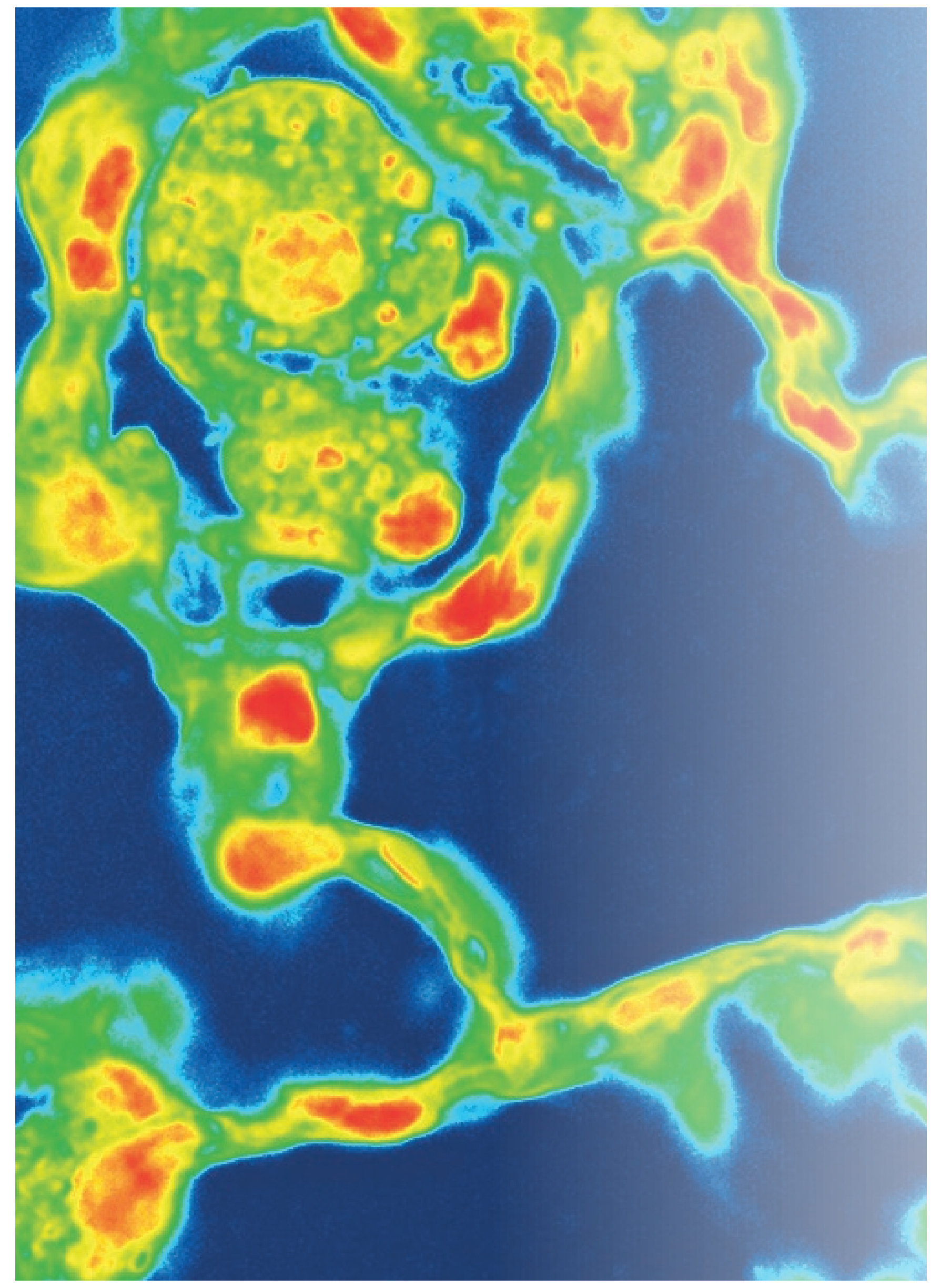




\section{Chapter 2}

\section{The effect of zirconium doping of cerium dioxide nanoparticles on pulmonary and cardiovascular toxicity and biodistribution in mice after inhalation}

Susan Dekkers' ${ }^{1}$ Mark R. Miller ${ }^{2}$, Roel P.F. Schins ${ }^{3}$, Isabella Römer ${ }^{4}$, Mike Russ ${ }^{5}$, Rob J. Vandebriel', Iseult Lynch4, Marie-France Belinga-Desaunay ${ }^{4}$, Eugenia Valsami-Jones ${ }^{4}$, Shea P. Connell', Ian P. Smith², Rodger Duffin ${ }^{2}$, John A.F. Boere', Harm J. Heusinkveld ${ }^{1,3}$, Catrin Albrecht ${ }^{3}$, Wim H. de Jong ${ }^{1}$, Flemming R. Cassee ${ }^{1,6}$

Nanotoxicology 1 1(6):794-808 (2017)

1 National Institute for Public Health and the Environment, Bilthoven, the Netherlands,

${ }^{2}$ Centre for Cardiovascular Science \& Centre for Inflammation Research, University of Edinburgh, Edinburgh, United Kingdom,

3 IUF- Leibniz Research Institute for Environmental Medicine, Düsseldorf, Germany,

${ }^{4}$ School of Geography, Earth and Environmental Sciences, University of Birmingham, Birmingham, United Kingdom,

${ }^{5}$ Promethean Particles Ltd, Nottingham, United Kingdom,

${ }^{6}$ Institute for Risk Assessment Sciences, Utrecht University, Utrecht, the Netherlands 


\section{Abstract}

Development and manufacture of nanomaterials is growing at an exponential rate, despite an incomplete understanding of how their physicochemical characteristics affect their potential toxicity. Redox activity has been suggested to be an important physicochemical property of nanomaterials to predict their biological activity. This study assessed the influence of redox activity by modification of cerium dioxide nanoparticles $\left(\mathrm{CeO}_{2} \mathrm{NPS}\right)$ via zirconium ( $\mathrm{Zr}$ ) doping on the biodistribution, pulmonary and cardiovascular effects in mice following inhalation.

Healthy mice (C57BL/6J), mice prone to cardiovascular disease (ApoE $\%$, western-diet fed) and a mouse model of neurological disease (5xFAD) were exposed via nose-only inhalation to $\mathrm{CeO}_{2}$ NPs with varying amounts of $\mathrm{Zr}$-doping $(0 \%, 27 \%$ or $78 \% \mathrm{Zr}$ ), or clean air, over a 4-week period ( $4 \mathrm{mg} / \mathrm{m}^{3}$ for $3 \mathrm{~h} /$ day, 5 days/week). Effects were assessed 4 weeks post exposure.

In all three mouse models $\mathrm{CeO}_{2} \mathrm{NP}$ exposure had no major toxicological effects apart from some modest inflammatory histopathology in the lung, which was not related to the amount of $\mathrm{Zr}$-doping. In $\mathrm{ApoE}^{-/}$mice $\mathrm{CeO}_{2}$ did not change the size of atherosclerotic plaques, but there was a trend towards increased inflammatory cell content in relation to the $\mathrm{Zr}$ content of the $\mathrm{CeO}_{2}$ NPs.

These findings show that subacute inhalation of $\mathrm{CeO}_{2}$ NPs causes minimal pulmonary and cardiovascular effects 4 weeks post exposure and that $\mathrm{Zr}$-doping of $\mathrm{CeO}_{2} \mathrm{NPs}$ has limited effect on these responses. Further studies with nanomaterials with a higher inherent toxicity or a broader range of redox activities are needed to fully assess the influence of redox activity on the toxicity of nanomaterials. 


\section{Introduction}

The amount and variety of nanomaterials developed, manufactured and used in different applications and commercial products is growing rapidly. Nanomaterials are of great interest because their physicochemical properties can be optimized to enhance their functionality in many different applications. However, the physicochemical properties that make nanomaterials so interesting may also lead to unexpected behavior and possible harmful effects in the environment and humans. To be able to better assess the potential risk of nanomaterials, more knowledge is needed on the possible toxicological consequences of their different (physicochemical) properties and their mechanisms of toxicity.

One of the key mechanisms of toxicity for nanomaterials is believed to be their ability to generate reactive oxygen species (ROS) (Unfried et al., 2007, Miller et al., 2012). After saturation of compensatory mechanisms, excessive cellular ROS can lead to oxidative stress, resulting in detrimental effects on cell function. In addition, amplification of inflammatory responses may occur that can exacerbate a number of diseases, such as cancer, cardiovascular diseases, neurodegenerative diseases and diabetes (Pisoschi and Pop, 2015). Nano-sized metal oxides are known to facilitate the formation of ROS by depleting electrons from cellular redox species (cellular components able to release electrons) or by serving as catalysts in ROS production through the Fenton reactions, Fenton-like reactions, or the Haber-Weiss cycle reaction (Fu et al., 2014).

While there are clear associations between ROS generation and the toxicity of nanomaterials, comparisons between nanomaterials are complicated by the use of different core chemistries that will manifest as differences in other physicochemical properties in addition to redox activity. In the current study we applied chemical doping (intentional substitution of one element by another while maintaining the lattice structure and arrangement) of a single type of nanoparticle to specifically investigate the influence of redox activity on ROS formation, and associated induction of oxidative stress responses in mice in vivo after inhalation exposure. $\mathrm{CeO}_{2}$ was selected as a nanomaterial that could be chemically modified appropriately and for which acute and subacute toxicity data from inhalation exposure was available (Srinivas et al., 2011, Demokritou et al., 2013, Aalapati et al., 2014, Gosens et al., 2014, Keller et al., 2014, Morimoto et al., 2015).

$\mathrm{CeO}_{2}$ NPs are used in variety of applications, such as electrochemical sensors, fuel cells, reforming catalysts, as well as a diesel fuel additive used to increase engine efficiency and reduce particulate exhaust emissions (Cassee et al., 2011, Cassee et al., 2012, Dunnick et al., 2016). Previous in vitro studies using pure $\mathrm{CeO}_{2} \mathrm{NPs}$ have shown contradicting results in terms of the oxidative stress impacts of $\mathrm{CeO}_{2}$ NPs (Dunnick et al., 2015, Leung et al., 2015, Pesic et al., 2015). Some studies have found $\mathrm{CeO}_{2} \mathrm{NPs}$ to induce ROS and oxidative stress 
(Lin et al., 2006, Park et al., 2008, Eom and Choi, 2009), while others show protective effects against oxidant-induced apoptosis (Xia et al., 2008, Celardo et al., 2011). Differences in the $\mathrm{CeO}_{2}$ NPs valence state in terms of the $3^{+}: 4^{+}$ratio, or the proportion of the valence state at the NP surface, could account for some of these inconsistencies. Ce has the ability to shift between a $3^{+}$and $4^{+}$valence state. The antioxidant properties of $\mathrm{CeO}_{2} \mathrm{NPs}$ are due to its ability to scavenge free radicals, which is accomplished through its ability to switch from the $3^{+}$to the $4^{+}$valence state (Hirst et al., 2009). The antioxidant efficacy of $\mathrm{CeO}_{2} \mathrm{NPs}$ can be enhanced by incorporation of $\mathrm{Zr}$ in the $\mathrm{CeO}_{2}$ lattice (Tsai et al., 2008).

In this study different quantities of Zr were incorporated into the crystalline structure of the $\mathrm{CeO}_{2} \mathrm{NPs}$ in an attempt to generate $\mathrm{CeO}_{2} \mathrm{NPs}$ with different antioxidant potentials. The Zr-doped $\mathrm{CeO}_{2}$ NPs were produced through an industrial collaboration and therefore had a real-life application. Furthermore, the $\mathrm{CeO}_{2}$ structure could tolerate $\mathrm{Zr}$ substitution at a range of concentrations, allowing for a precise control of the particle redox state. The aim of our study was to investigate if the modification of the redox activity of $\mathrm{CeO}_{2} \mathrm{NPs}$ by $\mathrm{Zr}$ doping influences their pulmonary and cardiovascular effects in mice following subacute inhalation exposure. We hypothesized that the adverse biological effects of $\mathrm{CeO}_{2} \mathrm{NPs}$ can be diminished by Zr-doping, due to an increased antioxidant potential of the doped nanoparticles.

The study was conducted in three different mouse models to explore the (patho)physiological effects of nanoparticle exposure on multiple organ systems. This paper describes the biodistribution, pulmonary and cardiovascular findings whereas the neurological data will be published in a separate paper.

\section{Materials and methods}

\section{Study design}

To explore the (patho)physiological effects of nanoparticle on multiple organ systems, three different mouse models were exposed.

Atherosclerosis-prone apolipoprotein E-deficient $\left(\mathrm{ApoE}^{-/-}\right)$mice are a well-established model for the study of the vascular disease atherosclerosis (Coleman et al., 2006), a disease characterized by the build-up of lipid- and inflammatory cell-rich plaques within arteries, which underlies the majority of cardiovascular diseases. The 4-week exposure protocol was integrated into an 8-week high-fat feeding regime that has been shown to generate complex atherosclerotic plaques with many of the hallmarks of the human disease in specific arterial locations (Cassee et al., 2012, Miller et al., 2013). ApoE ${ }^{-/-}$mice were used to study hematology, pulmonary and cardiovascular effects. 
The 5xFAD mice are an Alzheimer's disease mouse model. Although these mice were included to study neurological effects that will be published in a separate paper, the hematology and pulmonary effects were also studied within the same animals and reported in this paper.

C57BL/6J mice were used as the background (non-genetically modified) strain of the disease mouse models and used to study biodistribution, hematology and pulmonary effects.

Hematology, pulmonary and cardiovascular effects were assessed 4 weeks post exposure. This period was included, firstly, to provide the extra four weeks necessary for mice to develop sufficient plaque formation in arteries and secondly, to investigate the persistency of the pulmonary and cardiovascular effects.

Three groups of each mouse model were exposed to $\mathrm{CeO}_{2} \mathrm{NPs}$ with different amounts of $\mathrm{Zr}$-doping and one control group to clean air. The number of animals per group was different for each of the mouse models, to provide sufficient statistical power to detect differences between exposed and control animals in the most important effect parameter of each mouse model. Based on previous experiments, $8 \mathrm{ApoE}^{-/}$mice per group were expected to be sufficient to detect statistically significant differences in atherosclerotic plaque size and $5 \mathrm{C} 57 \mathrm{BL} / 6 \mathrm{~J}$ mice per group were expected to be sufficient to detect statistically significant differences in the number of neutrophils in the bronchoalveolar lavage fluid (BALF). However, $10 \mathrm{C} 57 \mathrm{BL} / 6 \mathrm{~J}$ mice per group were included, since $10 \mathrm{C} 57 \mathrm{BL} / 6 \mathrm{~J}$ mice per group and 16 5xFAD mice per group were needed to provide sufficient statistical power in the neurological study. Mice were exposed nose-only for four weeks to $4 \mathrm{mg} / \mathrm{m}^{3} \mathrm{NP}$ for 5 days/ week for 3 hours/day. This dose was primarily based on another 4-week inhalation study in which inflammatory responses in the lungs of CD-1 mice were observed after exposed to $2 \mathrm{mg} / \mathrm{m}^{3} \mathrm{CeO}_{2} \mathrm{NPs}$ for 5 days/week for 6 hours/day (Aalapati et al., 2014). Effects were assessed 4 weeks post the final exposure ( 56 days after the initial exposure). A schematic overview of the experimental design is presented in Figure 1.

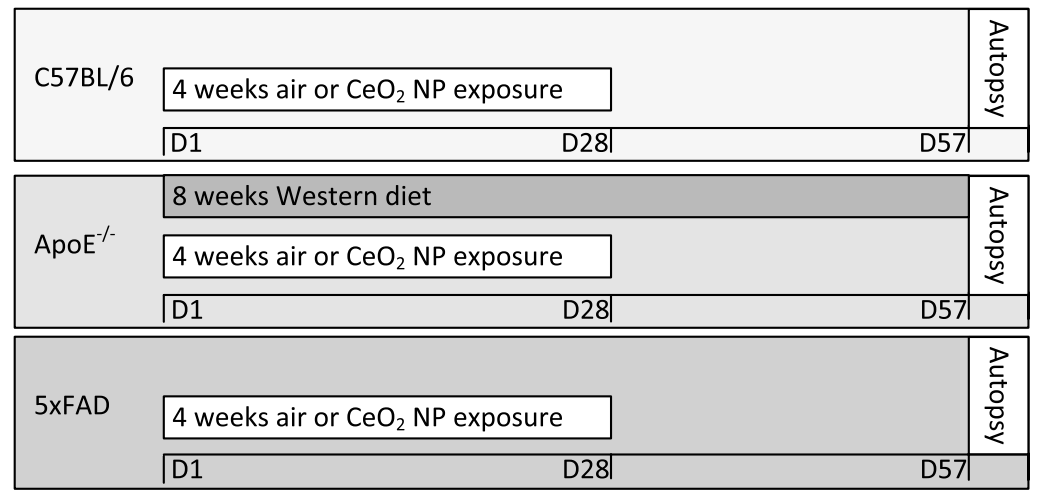

Figure 1: Schematic overview of the study design. $D=$ day. D1-D28 = exposure period and D29-D57 = recovery period. 


\section{Nanomaterial production and characterization}

To modify the redox activity, $\mathrm{CeO}_{2}$ NPs were doped with different amounts of $\mathrm{Zr}$ (as per Table 1, Zr contents in the doped NPs were $27 \mathrm{~mol} \%$ and $78 \mathrm{~mol} \%$ ). Addition of Zr into the crystalline structure of $\mathrm{CeO}_{2} \mathrm{NPs}$ is expected to enhance the antioxidant efficacy of $\mathrm{CeO}_{2} \mathrm{NPs}$ (Tsai et al., 2008).

$\mathrm{CeO}_{2} \mathrm{NPs}$ and $\mathrm{Zr}$-doped $\mathrm{CeO}_{2}$ NPs were produced using supercritical water hydrothermal synthesis (Cabanas et al., 2000). Briefly, $\mathrm{H}_{2} \mathrm{O}$ was pumped through a pre-heating coil, heated to approximately $400{ }^{\circ} \mathrm{C}$, brought into contact with a concurrently flowing solution of metal salts at room temperature (RT), while maintaining the flow rates, temperature and pressure constant. The mixture was cooled immediately after the mixing point and passed through a 7- $\mu \mathrm{m}$ filter to remove large aggregates. The solids in the aqueous suspensions were recovered by allowing the suspension to settle and drying at approximately $100^{\circ} \mathrm{C}$. The NPs were tested for endotoxin contamination using a Limulous Amoebocyte Lysate (LAL) test. No endotoxin was detected in any of the NP suspensions.

Table 1: Physicochemical characteristics of the nanoparticles.

\begin{tabular}{|c|c|c|c|c|}
\hline \multirow[t]{2}{*}{ Characteristic } & \multirow[t]{2}{*}{ Method } & \multicolumn{3}{|c|}{ Nanoparticle } \\
\hline & & $\mathrm{CeO}_{2}$ & $\begin{array}{l}27 \% \mathrm{Zr}^{\mathrm{Z}-\text { doped }} \\
\mathrm{CeO}_{2}\end{array}$ & $\begin{array}{c}78 \% \text { Zr-doped } \\
\mathrm{CeO}_{2}\end{array}$ \\
\hline $\begin{array}{l}\text { Primary particle size } \\
\text { (Mean } \pm \text { SD) }(\mathrm{nm})\end{array}$ & $\begin{array}{l}\text { STEM- } \\
\text { EELS }\end{array}$ & $4.7+1.4$ & $4.6+1.4$ & $4.7+1.4$ \\
\hline $\mathrm{Ce}^{3+} / \mathrm{Ce}^{4+}$ ratio & UV-vis & 0.87 & 0.98 & 1.4 \\
\hline $\begin{array}{l}\text { Scavenging capacity ROS (Mean } \pm \text { SD) (\% reducti- } \\
\text { on compared to signal without NPs) }\end{array}$ & ESR & $46 \pm 26$ & $44 \pm 21$ & $56 \pm 23$ \\
\hline $\begin{array}{l}\text { Median particle diameter at } 128 \mu \mathrm{g} / \mathrm{ml} \text { in water } \\
\text { (Mean } \pm \text { SD) }(\mathrm{nm})\end{array}$ & DCS & 39 & 40 & 41 \\
\hline $\begin{array}{l}\text { Median particle diameter at } 20 \mu \mathrm{g} / \mathrm{ml} \text { in water } \\
(\text { Mean } \pm \mathrm{SD})(\mathrm{nm})\end{array}$ & DLS & $54 \pm 9$ & $45 \pm 5$ & $52 \pm 6$ \\
\hline $\begin{array}{l}\text { Median particle diameter at } 1 \mathrm{mg} / \mathrm{ml} \text { in water } \\
(\text { Mean } \pm \text { SD) }(\mathrm{nm})\end{array}$ & DLS & $\begin{array}{c}172 \pm 2 \\
\text { PDI: } 0.27 \pm \\
0.01\end{array}$ & $\begin{array}{c}297 \pm 4 \\
\text { PDI: } 0.47 \pm \\
0.01\end{array}$ & $\begin{array}{c}358 \pm 6 \\
\text { PDI: } 0.49 \pm \\
0.03\end{array}$ \\
\hline zeta potential at $1 \mathrm{mg} / \mathrm{ml}$ in water $(\mathrm{mV} \pm \mathrm{SD})$ & DLS & $50.3 \pm 0.7$ & $45.9 \pm 3$ & $44.1 \pm 2$ \\
\hline $\mathrm{EC}_{20}$ cytotoxicity in $\mathrm{A} 549($ Mean $\pm 90 \% \mathrm{Cl})\left(\mu \mathrm{g} / \mathrm{cm}^{2}\right)$ & WST-1 & $0.2(0.0-23)$ & $9(0.0-38)$ & $33(0.1-68)$ \\
\hline
\end{tabular}

STEM-EELS: Scanning Transmission Electron Microscope (STEM) Electron Energy Loss Spectroscopy (EELS) analysis; UV-vis: Ultraviolet-Visible spectrophotometry; ESR: Electron-Spin Resonance; DCS: Differential Centrifugal Sedimentation; DLS: Dynamic Light Scattering; WST-1: Water-Soluble Tetrazolium-1; EC ${ }_{20}$ : Effective Concentration resulting in $20 \%$ cytotoxicity.

To determine the $\mathrm{Ce}^{3+} / \mathrm{Ce}^{4+}$ ratio of the different NPs, ultraviolet-visible spectrophotometry (UV-vis) analysis was performed on $200 \mathrm{mg} / \mathrm{L}$ particle solutions, diluted in MilliQ water, with a 6800 Jenway double beam UV-Vis spectrophotometer, with long path UV-Vis cell cuvettes. The UV-Vis absorption spectra were collected over a wavelength range of $200-700 \mathrm{~nm}$. Both valence states of Ce strongly absorb ultraviolet light and each one has a characteristic 
spectrophotometric absorbance peak, with $\mathrm{Ce}^{3+}$ absorbing in the 230 to $260 \mathrm{~nm}$ range and $\mathrm{Ce}^{4+}$ absorbing in the 300 to $400 \mathrm{~nm}$ range.

Zr-doping of $\mathrm{CeO}_{2}$ NPs might also cause differences in other characteristics than redox activity which can also influence the toxicity of the NPs, such as particle size distribution, aggregation, agglomeration and corona formation. Therefore the hydrodynamic size distributions and zeta potential were assessed using differential centrifugal sedimentation (DCS) and dynamic light scattering (DLS) analyzes. In addition, the effect of Zr-doping on the scavenging capacity of the $\mathrm{CeO}_{2} \mathrm{NPs}$ was investigated with Electron-spin resonance (ESR). Before testing the NPs in vivo, the effect $\mathrm{Zr}$-doping of $\mathrm{CeO}_{2} \mathrm{NPs}$ on the in vitro cytotoxicity was investigated in lung epithelial cells.

The scavenging capacity of ROS was investigated with ESR analysis using a cell free system with a 5,5-dimethyl-1-pyrroline N-oxide (DMPO) spin trap (He et al., 2014) in combination with hydrogen peroxide $\left(\mathrm{H}_{2} \mathrm{O}_{2}\right)$ and copper sulfate $\left(\mathrm{CuSO}_{4}\right)$. The nanoparticle suspensions were thoroughly vortexed and sonicated for 5 minutes at RT in a sonication bath to redisperse any agglomerates. A sample was made of $12.5 \mu \mathrm{L}$ nanoparticle suspension (1.28 $\mathrm{mg} / \mathrm{mL}), 12.5 \mu \mathrm{LCuSO}_{4}(20 \mu \mathrm{M}), 25 \mu \mathrm{L} \mathrm{H}_{2} \mathrm{O}_{2}(0.5 \mathrm{M})$ and $50 \mu \mathrm{L}$ DMPO $(0.05 \mathrm{M})$. This sample was incubated in a shaker water bath at $37^{\circ} \mathrm{C}$ for 15 minutes at $100 \mathrm{rpm}$, vortexed and taken up in a capillary tube which was then sealed at the bottom with hematocrit and measured with the ESR Spectroscope (Miniscope MS 400, MT MagnetTech GmbH).

The effect of Zr-doping on in vitro cytotoxicity was tested in human lung epithelial carcinoma cells (A549) cells obtained from ATCC (VA, USA). A549 cells were cultured in tissue culture flasks in RPMI 1640 medium with Glutamax (Gibco, ThermoFisher Scientific Inc., Landsmeer, the Netherlands) supplemented with 10\% Fetal Bovine Serum (FBS, Greiner BioOne BV, Alphen aan de Rijn, the Netherlands) and $1 \%$ penicillin/streptomycin (Gibco). Cells were cultured at $37^{\circ} \mathrm{C}$ in a humidified atmosphere of $5 \% \mathrm{CO}_{2}$ in air. Twenty-four hours before exposure, the cells were harvested by a short incubation with $0.5 \%$ ethylenediaminetetraacetic acid (EDTA) trypsin in Ca/Mg free Dulbecco's Phosphate Buffered Saline (Gibco) and counted. Fifty thousand $\left(5 \times 10^{4}\right)$ cells were seeded in 96 -well plates in $200 \mu \mathrm{L}$ cell culture medium per well. After $24 \mathrm{~h}$ of incubation a semi confluent monolayer of cells was obtained and the cells were exposed to $0,1.252 .5,5,10,20,40$ or $80 \mu \mathrm{g} / \mathrm{cm}^{2}$ of the various NPs in cell culture medium. Additional wells with the same concentrations of NPs in cell culture medium, but no A549 cells were included to measure possible interference of the materials with the viability assay. Cell viability (i.e. cytotoxicity) was determined after $24 \mathrm{~h}$ of exposure by a colorimetric assay using cell proliferation reagent WST-1 (water-soluble tetrazolium-1) (Roche, Sigma-Aldrich Chemie). Dose-response modelling and derivations of the $\mathrm{EC}_{20}$ (effective concentration resulting in $20 \%$ cytotoxicity) were performed using PROAST software (Slob, 2002) version 63.5 . 


\section{Animals}

Specific pathogen free (SPF) female ApoE ${ }^{-/-}$mice ( $\mathrm{N}=32$; Taconic, Denmark) were obtained at age 10-12 weeks at the beginning of the study. The 5xFAD and C57BL/6J mice originated from Jackson Laboratories. For this study, female 5xFAD mice $(\mathrm{N}=64)$ and female wild type (WT) cross bread C57BL/6J littermates $(N=40)$ were used at the age of 8-11 weeks. The mice were barrier maintained and housed in a single room in macrolon cages. The temperature and the relative humidity were controlled at $22 \pm 2^{\circ} \mathrm{C}$ and at $40-70 \%$, respectively. Lighting was artificial with a sequence of 12 hours light and 12 hours dark (at night). Feed and drinking water was provided ad libitum from the arrival of the mice until the end of the study, except during exposure. The ApoE $\mathrm{E}^{-/}$mice were fed a commercially available rodent Western (high fat) diet (Purified Diet Western 4021.06, ABdiets, Woerden, The Netherlands), starting at the first day of the exposure period until the end of the experiment. The other mice were fed a standard commercially available rodent diet (SMR-A, ABdiets, Woerden, The Netherlands). Animals were monitored by cage-side observations and, if necessary, handled to detect signs of compromised health. The body weight of each animal was recorded one day before the start of exposure (day -1), prior to exposure on the first day and weekly thereafter. Experiments were conducted at Intravacc (Bilthoven, The Netherlands) under a protocol approved by the Ethics Committee for Animal Experiments of the RIVM and performed according to applicable national and EU regulations.

\section{Inhalation exposure}

Approximately one week before the 4-week exposure period, 20 samples of each nanoparticle (one for each day) with a concentration of $1 \mathrm{mg} / \mathrm{mL}$ were prepared from the stock dispersions (20, 20 or $29 \mathrm{mg} / \mathrm{mL}$ for $0 \%, 27 \%$ and $78 \% \mathrm{Zr}$-doped $\mathrm{CeO}_{2}$ NPs, respectively) by diluting with ultrapure water to the desired concentration. Stock and sample dispersions were sonicated for 5 minutes in an ultrasonic bath (Branson CPX2800, $40 \mathrm{kHz}, 110 \mathrm{~W}$ ) before use to redisperse any possible agglomerates. Freshly generated aerosols of NPs were generated using a spray nozzle technique, diluted with pressurized clean particle-free air, and heated to 24 $25^{\circ} \mathrm{C}$. Exposure was controlled based on stable particle number counts, mass concentrations, temperature and relative humidity, measured continuously using a condensation particle counter (CPC 3022A from TSI Inc., St. Paul, MN, USA), a tempered element oscillating microbalance (TEOM series1400A from Rupprecht \& Patashnick, NY, USA) and M-170 Measurement Indicator (Vaisala M170, Vaisala Oyj, Helsinki, Sweden), respectively, during each exposure period. In addition, the test atmosphere is characterized at least twice during each exposure session using an optical particle sizer (OPS 3330, TSI Inc., St. Paul, MN, USA) and a scanning mobility particle sizer (SMPS 3936 from TSI Inc., St. Paul, MN, USA). The total mass concentration generated over the 3-hour exposure period was determined by gravimetric analysis of pre-weighed and post-weighed polytetrafluoroethylene (PTFE) filters (Teflo R2PJO47, Pall corporation, Port Washington, New York, USA) using a micro-balance (Mettler MC or ME-5 microbalance, Mettler-Toledo LLC, Columbus, OH, USA). The Count 
Median Diameter (CMD) and the Mass Median Diameter (MMD) were estimated using the Aerosol Instrument Manager Software (Release Version 9.0.0.0, 15:32:53, Nov 112010 from TSI Inc., St Pauls, MN, USA), assuming spherical aggregation around primary particles of $4.7 \pm 1.4 \mathrm{~nm}$. In addition, aerosols were collected on polycarbonate filters for scanning electron microscopy (SEM) analysis. The SEM samples were prepared by placing a small piece of the filter on the SEM stub and coating it with platinum, and visualizing with an XL30 Environmental SEM-FEG microscope (Philips XL30 ESEM-FEG). During the 3 hour exposure periods to the different nanoparticles, the control groups were exposed to filtered air under the same conditions (nose-only tubes) for the same amount of time.

\section{Estimated deposited dose in lungs}

To estimate the deposited dose in the lungs, the multiple path particle dosimetry model (MPPD v3.04 @ 2016 by Applied Research Associates, Inc., Albuquerque, NM, USA) was used. Default parameters for the B6C3F1 mouse were applied (forced respiratory capacity: $0.3 \mathrm{~mL}$, upper respiratory tract volume: $0.0322 \mathrm{~mL}$, nasal breathing, breathing frequency: $353 / \mathrm{min}$, tidal volume: $0.20 \mathrm{~mL}$, inspiration fraction: 0.5 , no pause fraction). Calculations were performed using the count median diameter, geometric standard deviation and mass concentration of the exposure characterization and a density of $7.215 \mathrm{~g} / \mathrm{cm}^{3}$ for $\mathrm{CeO}_{2}, 6.801$ $\mathrm{g} / \mathrm{cm}^{3}$ for $27 \% \mathrm{Zr}$-doped $\mathrm{CeO}_{2}$ and $6.018 \mathrm{~g} / \mathrm{cm}^{3}$ for $78 \% \mathrm{Zr}$-doped $\mathrm{CeO}_{2} \mathrm{NPs}$.

\section{Quantification of Ce and Zr in tissues}

During necropsy, organs from half of the C57BL/6J mice per group were obtained to evaluate the distribution of the NPs throughout the body. Liver, spleen, kidneys, heart and right (exposed mice) or left (control mice) lung, were weighed and immediately frozen in liquid nitrogen for determination of the $\mathrm{Ce}$ and $\mathrm{Zr}$ concentrations. To allow measurement of multiple parameters within the same animal, different parts of the lungs were selected for the exposed compared to the control animals. From the exposed groups the right lung was used for quantification of $\mathrm{Ce}$ and $\mathrm{Zr}$, because the left lung was needed for histopathological examination. From the control group the left lung was used for quantification of $\mathrm{Ce}$ and $\mathrm{Zr}$, because the right lung was needed for bronchoalveolar lavage. The organs were digested by acidification of each sample with $2 \mathrm{~mL}$ nitric acid for $12 \mathrm{~h}$. Hydrogen fluoride $(0.2 \mathrm{~mL})$ was added, followed by microwave heating for $45 \mathrm{~min}$ up to $185^{\circ} \mathrm{C}$, and maintained for a further $20 \mathrm{~min}$. Boric acid $(2 \mathrm{~mL}$ ) was added to neutralize the hydrogen fluoride, and the samples were re-heated for $20 \mathrm{~min}$ to $160^{\circ} \mathrm{C}$, and maintained for $10 \mathrm{~min}$. Once cooled, samples were filtered with a $450 \mathrm{~nm}$ syringe filter, diluted with $10 \mathrm{~mL}$ deionized water and stored at room temperature (RT). The presence of Ce and $\mathrm{Zr}$ in the lungs, liver, spleen, kidneys and heart was determined by inductively coupled plasma mass spectrometry (ICP-MS) using a Perkin Elmer NexION 300X instrument operated in standard mode for Ce and $\mathrm{Zr}$. The isotopes measured were ${ }^{90} \mathrm{Zr}$ and ${ }^{140} \mathrm{Ce}$ using ${ }^{115} \mathrm{In}$ and ${ }^{159} \mathrm{~Tb}$ as internal standards. Calibration standards $(0-100$ 
$\mu \mathrm{g} / \mathrm{L}$ ) were prepared from VWR $1000 \mathrm{mg} / \mathrm{L}$ stock solutions. Quantities are expressed as $\mu \mathrm{g} / \mathrm{g}$ organ tissue.

\section{Necropsy \\ Hematology}

Animals were anaesthetized with a mixture of ketamine and xylazine. Two blood samples were taken by eye extraction. The first sample was collected in a K3-EDTA tube (Minicollect K3EDTA, $1 \mathrm{~mL}, 450474$ Greiner Bio-One) for hematological parameters as determined in a blood auto analyzer (ADVIA 2120 Hematology System, Siemens Healthineers) within 3 hours after collection. The second sample was collected in a serum tube, which was kept for at least 30 minutes at RT before centrifugation at $2000 \mathrm{~g}$ for $10 \mathrm{~min}$ at $20^{\circ} \mathrm{C}$. Serum was divided into separate aliquots of $50 \mu \mathrm{L}$ and stored at $-20^{\circ} \mathrm{C}$ for further analysis.

\section{Bronchoalveolar lavage}

Lung lavage was performed at necropsy. A cannula was placed in the trachea and the diaphragm opened to decrease the amount of air inside the lungs. For the control animals and all $\mathrm{ApoE}^{-/-}$animals, the right lung half was rinsed twice with approximately $0.5 \mathrm{~mL}(26.7$ $\mathrm{mL}$ per kg body weight) of physiological saline solution, after ligation of the left lung. The injected volume was inserted and recovered 3 times, after which the lavage liquid was collected and stored on ice for less than 2 hours. To allow necropsy of the planned number of animals within one day, both lungs were lavaged for 10 of the 16 exposed 5xFAD mice and 5 of the 10 exposed $\mathrm{C57BL} / 6 \mathrm{~J}$ mice, using the same procedure, but approximately 0.8 $\mathrm{mL}$ (40 $\mathrm{mL}$ per kg body weight) of physiological saline solution. BALF was centrifuged at $400 \mathrm{~g}$ for 10 minutes at $4^{\circ} \mathrm{C}$. The supernatant was divided into two separate aliquots of 125 $\mu \mathrm{L}$ for total protein (TP; an indicator for acute lung injury), lactate dehydrogenase (LDH; an indicator of cytotoxicity), gamma-glutamyl transferase (GGT; an indicator of lung cell damage) and alkaline phosphatase (ALP; an indicator of type II cell damage) measurements using an autoanalyser (LX20-Pro, Beckman-Coulter, Woerden, the Netherlands). The cell pellet was scored for the presence of erythrocytes, re-suspended in $500 \mu \mathrm{L}$ phosphatebuffered saline (PBS) and kept on ice. Cell counts were determined in the re-suspended pellet using a Coulter counter (Beckman-Coulter, Live Sciences, Woerden, the Netherlands). Cell concentrations were determined using a single sample using at least $150 \mu \mathrm{L}$ of the resuspended cells. Cytospins (Cytospin 3, Thermo-Shandon, Runcorn, UK) were prepared and stained using May-Grunwald and Giemsa stain, and cell differentiation was performed by counting 400 cells per slide. 


\section{Histopathology}

Lung tissue. For the majority of the animals, the right lung was removed after the collection of the BALF and immediately frozen in liquid nitrogen and stored at $-80^{\circ} \mathrm{C}$ for further analysis. The left lung was removed and, after weighing, cannulated and infused with formaldehyde for 1 hour at a pressure of $20 \mathrm{~cm} \mathrm{H}_{2} \mathrm{O}$. Lungs were processed for histopathology; embedded in paraffin wax, sectioned at a thickness of 2-4 $\mu \mathrm{m}$ and stained with hematoxylin and eosin for histopathological examination. Histopathological changes were described according to distribution, severity and morphological characteristics. The morphological characteristics of chronic inflammation include for example the presence of lymphocytes and macrophages in the lung tissue, while acute inflammation is characterized by the presence of polymorphoneclear neutrophils (PMNs). Severity scores were assigned as follows: Grade 1 minimal/very few/very small; Grade 2 slight/few/small; Grade 3 moderate/ moderate number/moderate size; Grade 4 marked/many/large; Grade 5 massive/extensive number/extensive size.

To allow necropsy of the planned number of animals within one day, both lungs were lavaged for 10 of the 16 exposed 5xFAD mice and 5 of the 10 exposed C57BL/6J mice. For the remaining six of the 16 exposed 5xFAD mice both lungs were infused with formaldehyde and embedded in paraffin wax for histopathological examination. For the remaining five of the 10 exposed C57BL/6J mice the right lung was weighed and immediately frozen in liquid nitrogen for determination of the $\mathrm{Ce}$ and $\mathrm{Zr}$ concentrations and the left lung was infused with formaldehyde and embedded in paraffin wax for histopathological examination. For five of the 10 control $\mathrm{C} 57 \mathrm{BL} / 6 \mathrm{~J}$ mice the right lung was lavaged and stored at $-80^{\circ} \mathrm{C}$ and the left lung was weighed and immediately frozen in liquid nitrogen for determination of the $\mathrm{Ce}$ and $\mathrm{Zr}$ concentrations.

Assessment of atherosclerosis. Arteries (brachiocephalic, aortic arch, thoracic aorta) were isolated from ApoE ${ }^{-/-}$mice. Atherosclerosis was quantified, as previously published (Miller et al., 2013). Briefly, brachiocephalic arteries were fixed in formalin and histological sections were taken in triplicate at $100 \mu \mathrm{m}$ intervals, beginning at the first section of the artery with a fully intact media. Sections were stained with Masson's Trichrome. The cross-sectional area of the plaque was measured and standardized to the medial area. A single mean value for atherosclerotic burden for each animal was calculated from the plaque size from each complete serial section throughout the brachiocephalic artery. A single section from each artery (the section exhibiting the largest plaque in cross-section) was chosen for mac- 2 immunohistochemistry for macrophage-derived cells. A rat anti-mouse primary antibody was used (1/12000; CL8942AP, VH Bio, Gateshead, UK) with rat IgG (1/12000; I-400, Vector Labs, Peterborough, UK) as a negative control, followed by a goat anti-rat IgG biotinylated secondary antibody (BA-9400, Vector Labs). The area of positive staining was expressed as a proportion of the total plaque area. 
Other organs. The spleen, liver, heart and kidneys were removed, weighed and stored in $4 \%$ formaldehyde for pathological analysis if required based on macroscopic findings.

\section{Statistical analyses}

Statistical analyses were performed using GraphPad Prism v7.00 (GraphPad Software, San Diego, California, USA). Ordinary one-way analysis of variance (ANOVA) analyses including all experimental groups were performed followed by a Tukey's post-hoc multiple comparisons test. A p-value $\leq 0.05$ was considered statistically significant. If the group means indicated an increase or decrease with increasing amounts of Zr-doping, a linear trend analysis (alpha 0.1 ) was performed between the groups exposed to $0 \%, 27 \%$ and $78 \% \mathrm{Zr}$-doped $\mathrm{CeO}_{2} \mathrm{NPs}$, to investigate if observed effects could be related to the amount of $\mathrm{Zr}$ doping of the particles .

\section{Results}

\section{Nanoparticle characterization}

UV-vis analysis showed a clear trend of increasing $\mathrm{Ce}^{3+} / \mathrm{Ce}^{4+}$ ratio with increasing $\mathrm{Zr}$ content (see Figure 2 and Table 1). The DCS and DLS analysis both showed no significant differences in hydrodynamic size distributions (data not shown) or median particles size (see Table 1) of the different NPs, indicating the Zr-doping did not cause major differences in particle size distribution, aggregation or agglomeration. As expected, the zeta potential showed a small decrease with increasing amounts of Zr-doping.

\section{$\mathrm{Ce}^{3+} / \mathrm{Ce}^{4+}$ ratio $\mathrm{CeO}_{2} \mathrm{NPs}$ as measured with UV-vis}

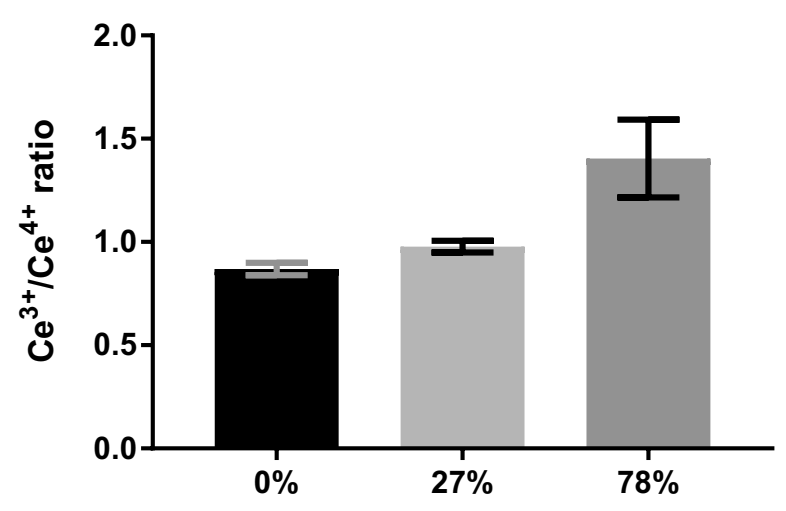

$\% \mathrm{Zr}$-doping of $\mathrm{CeO}_{2} \mathrm{NPs}$

Figure 2: $\mathrm{Ce}^{3+} / \mathrm{Ce}^{4+}$ ratio measured with UV-vis of undoped $\mathrm{CeO}_{2}, 27 \% \mathrm{Zr}$-doped $\mathrm{CeO}_{2}$ and $78 \% \mathrm{Zr}$-doped $\mathrm{CeO}_{2} \mathrm{NPs}_{\text {, }}$ showing an increasing $\mathrm{Ce}^{3+} / \mathrm{Ce}^{4+}$ ratio with increasing $\mathrm{Zr}$-content of $\mathrm{CeO}_{2} \mathrm{NPs}$. 
ESR analysis showed a slight, but not statistically significant increased scavenging capacity of the $\mathrm{CeO}_{2} \mathrm{NPs}$ with the highest \% of Zr-doping compared to the undoped $\mathrm{CeO}_{2} \mathrm{NPs}$ (see Figure 3 and Table 1).

In A549 cells, a decrease in cytotoxicity with increasing amounts of Zr-doping was shown. Dose response modelling resulted in increasing $\mathrm{EC}_{20}$ values with increasing amounts of $\mathrm{Zr}$ doping (see Figure 4 and Table 1).

\section{ESR measurements of the scavenging capacity of $\mathrm{CeO}_{2}$ and $\mathrm{Zr}$-doped $\mathrm{CeO}_{2} \mathrm{NPs}$}

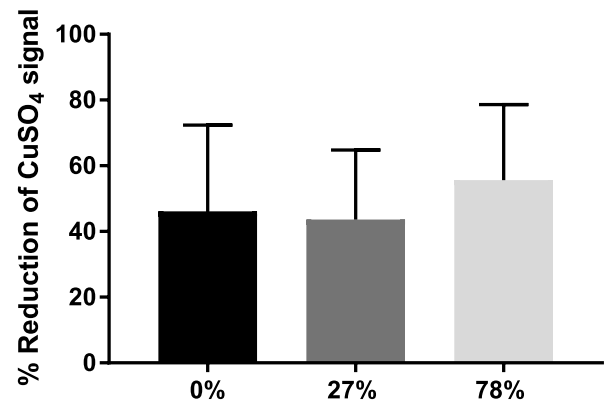

$\%$ Zr-doping of $\mathrm{CeO}_{2}$ NPs

Figure 3: Percentage reduction of the Electron-spin resonance (ESR) signal of $\mathrm{CuSO}_{4}$ and $\mathrm{CeO}_{2} \mathrm{NPs}_{\text {compared to }}$ $\mathrm{CuSO}_{4}$ alone using a cell free system with a 5,5-dimethyl-1-pyrroline $\mathrm{N}$-oxide (DMPO) spin trap in combination with $\mathrm{H}_{2} \mathrm{O}_{2}$. The slightly larger \% reduction of the $78 \% \mathrm{Zr}$-doped NPs indicates a slight but not statistically significant increase in scavenging capacity of reactive oxygen species (ROS) of $78 \% \mathrm{Zr}$-doped compared to the undoped and $27 \% \mathrm{Zr}$-doped $\mathrm{CeO}_{2}$ NPs.

a Cell viability A549 after $24 \mathrm{~h}$ exposure

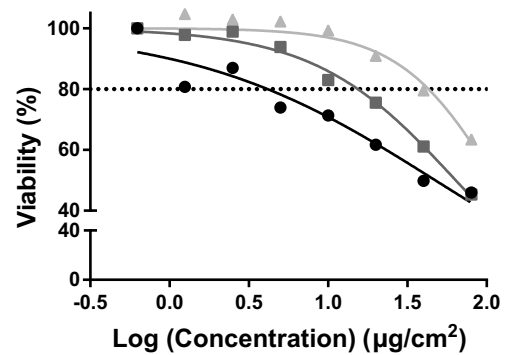

b EC $_{20}$ A549 after $24 \mathrm{~h}$ exposure

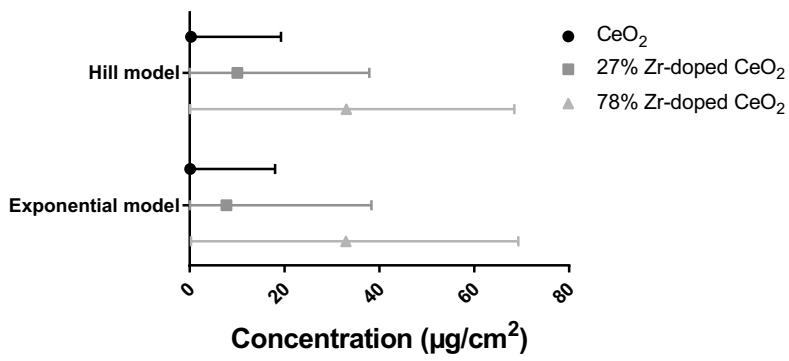

Figure 4: Percentage reduction in viability of A549 cells after exposure to $0,1.252 .5,5,10,20,40$ or $80 \mu \mathrm{g} / \mathrm{cm}^{2}$

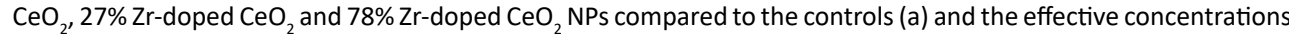
resulting in $20 \%$ cytotoxicity $\left(\mathrm{EC}_{20}\right)$ mean $\pm 90 \%$ confidence interval (b). 


\section{Exposure characterization}

The combined SMPS-OPS particle size measurements showed that almost the entire particle size distribution was within the size range measurable with the SMPS $(2.5-1000 \mathrm{~nm})$, confirming that the SMPS data can be used to estimate the CMD and MMD. A representative SMPS and OPS size distribution of each of the different $\mathrm{CeO}_{2} \mathrm{NPs}$ are shown in Figure 5.
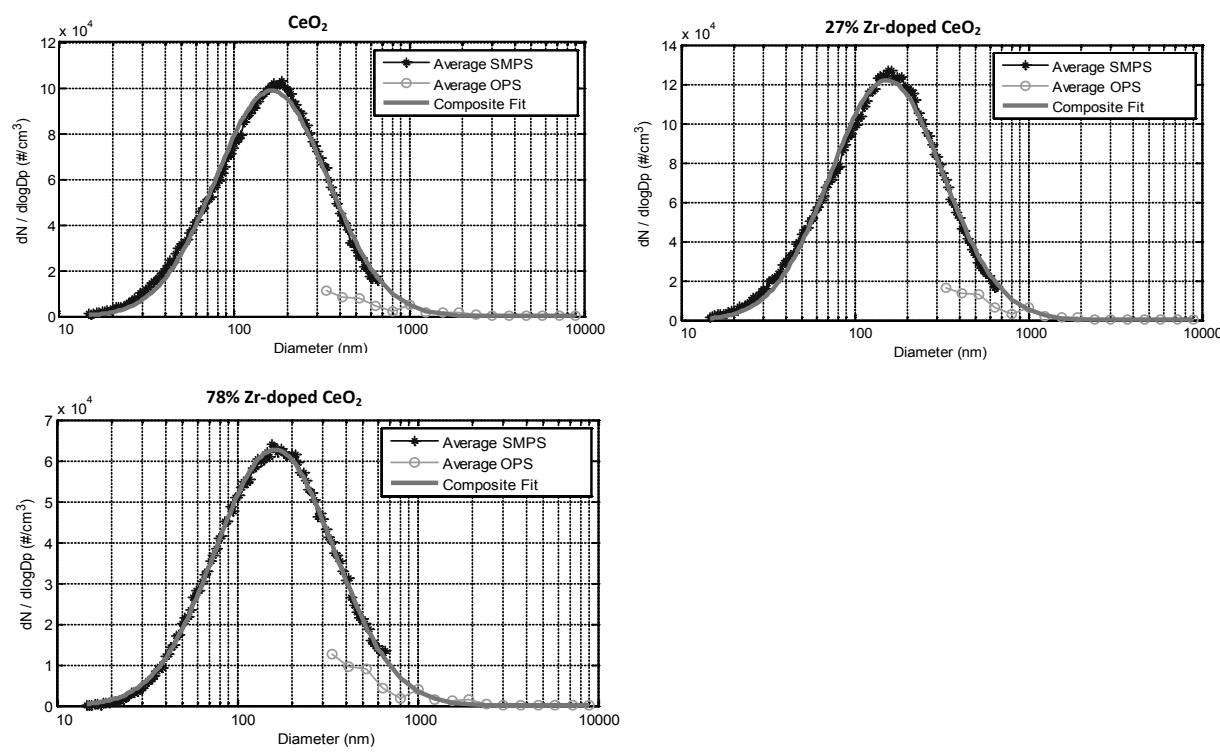

Figure 5: Combined size distributions measured with the scanning mobility particle sizer (SMPS) and the optical particle sizer (OPS) for representative samples of $\mathrm{CeO}_{2}, 27 \% \mathrm{Zr}$-doped $\mathrm{CeO}_{2}$ and $78 \% \mathrm{Zr}$-doped $\mathrm{CeO}_{2} \mathrm{NPs}$. The OPS distributions overlap with the composited fit of the SMPS distributions for the largest particles of the distributions, indicating that the SMPS measurements can be used to determine the count median diameter (CMD) and mass median diameter (MMD).

The physical characteristics of the different $\mathrm{CeO}_{2} \mathrm{NP}$ aerosols are summarized in Table 2 . The primary particle size of the different $\mathrm{CeO}_{2} \mathrm{NPs}$ was $\sim 4.7 \pm 1.4 \mathrm{~nm}$. CMD and MMD, as measured by SMPS, were slightly larger for the $78 \% \mathrm{Zr}$-doped $\mathrm{CeO}_{2}$ particles, compared to the $\mathrm{CeO}_{2}$ and $27 \% \mathrm{Zr}$-doped $\mathrm{CeO}_{2}$ particles. Mass concentration measurements determined the exposure concentration, thus, the particle number concentrations of the $78 \% \mathrm{Zr}$-doped $\mathrm{CeO}_{2}$ exposure were lower than the particle number concentrations of the $\mathrm{CeO}_{2}$ and $27 \%$ $\mathrm{Zr}$-doped $\mathrm{CeO}_{2}$ exposures.

SEM images of the NPs from aerosols collected on polycarbonate filters showed the presence of particles and agglomerates, when collected on the filter (see Figure 6). Most particles were around 300 to $500 \mathrm{~nm}$, but there were also infrequent very large particles (around 1000 to $2000 \mathrm{~nm}$ ). 
Table 2: Average particle number concentration, size distribution and mass concentration of the $\mathrm{CeO}_{2}, 27 \% \mathrm{Zr}$ doped $\mathrm{CeO}_{2}$ and $78 \% \mathrm{Zr}$-doped $\mathrm{CeO}_{2} \mathrm{NP}$ exposure $(\mathrm{n}=20)$.

\begin{tabular}{|c|c|c|c|c|c|}
\hline & & & $\mathrm{CeO}_{2}$ & $27 \% \mathrm{Zr}$-doped $\mathrm{CeO}_{2}$ & $78 \% \mathrm{Zr}$-doped $\mathrm{CeO}_{2}$ \\
\hline STEM & Primary particle size* & $\mathrm{nm}$ & $4.7+1.4$ & $4.6+1.4$ & $4.7+1.4$ \\
\hline $\mathrm{CPC}$ & Number concentration & $\# / \mathrm{cm}^{3}$ & $14624 \pm 3709$ & $16962 \pm 5145$ & $10785 \pm 894$ \\
\hline \multirow[t]{5}{*}{ SMPS } & Count Median Diameter (CMD) & $\mathrm{nm}$ & $182 \pm 10$ & $183 \pm 9$ & $202 \pm 7$ \\
\hline & Geometric Standard Deviation & & 1.88 & 1.88 & 1.86 \\
\hline & Mass Median Diameter (MMD)** & $\mathrm{nm}$ & $280 \pm 12$ & $288 \pm 9$ & $316 \pm 14$ \\
\hline & Geometric Standard Deviation & & 1.55 & 1.56 & 1.57 \\
\hline & Number concentration & $\# / \mathrm{cm}^{3}$ & $75620 \pm 28184$ & $70828 \pm 46924$ & $58716 \pm 20693$ \\
\hline Filter & Gravimetric mass concentration & $\mathrm{mg} / \mathrm{m}^{3}$ & $3.98 \pm 0.23$ & $4.04 \pm 0.30$ & $4.09 \pm 0.22$ \\
\hline
\end{tabular}

*Primary particle size was determined by Scanning Transmission Electron Microscope (STEM) Electron Energy Loss Spectroscopy (EELS) analysis.

**MMD was estimated based on the particle size distributions of the SMPS measurements, assuming spherical aggregation around primary particles of $4.7 \mathrm{~nm}$.

CPC: Condensation Particle Counter; SMSP: Scanning Mobility Particle Sizer.
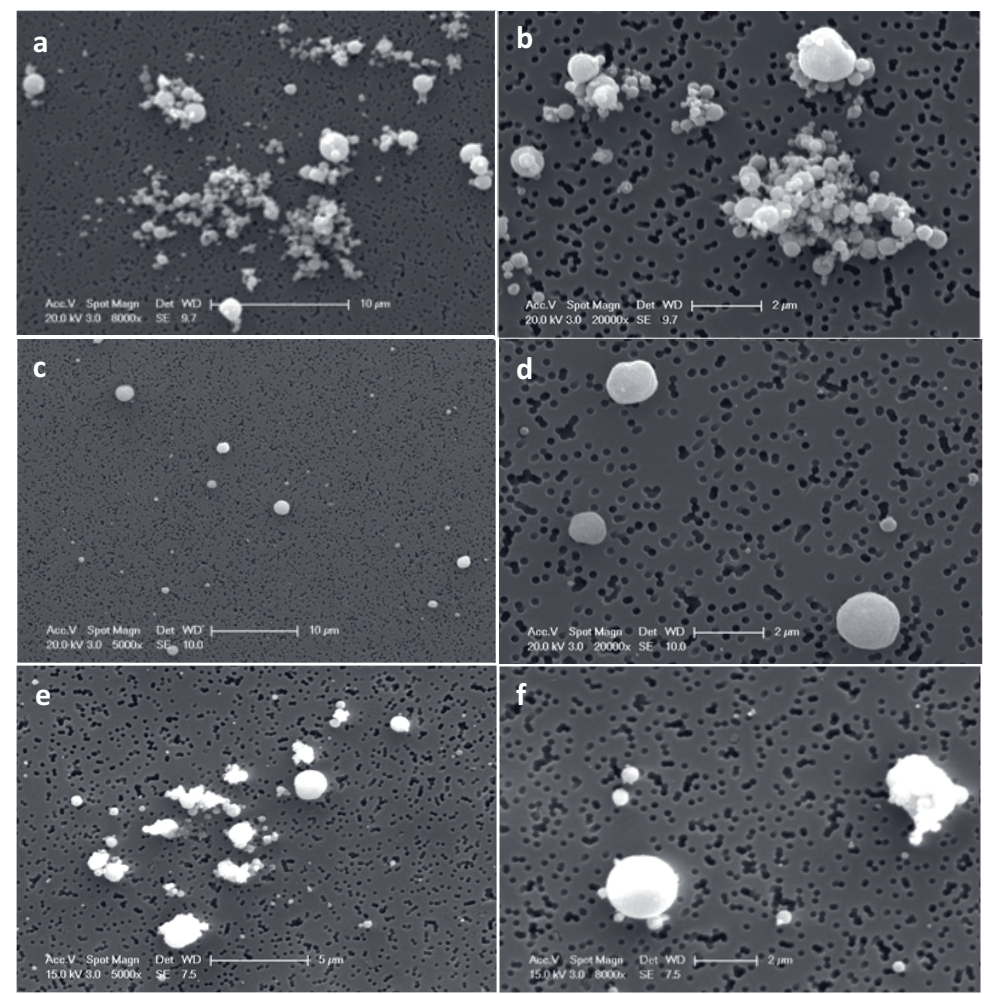

Figure 6: Scanning Electron Microscopy (SEM) image of $\mathrm{CeO}_{2}$ particles from aerosols collected on polycarbonate filters. Undoped $\mathrm{CeO}_{2} \mathrm{NPs}$ ( $\mathrm{a}$ and $\mathrm{b}$ ) showed the presence of agglomerates mostly around 300 to $400 \mathrm{~nm}$, but also some infrequent very large particles around $2000 \mathrm{~nm}$. 27\% Zr-doped $\mathrm{CeO}_{2} \mathrm{NPs}$ (c and d) showed no agglomerates and particles were mostly around $500 \mathrm{~nm}$. 78\% $\mathrm{Zr}$-doped $\mathrm{CeO}_{2} \mathrm{NPs}$ showed the presence of agglomerates, mostly around $400 \mathrm{~nm}$, but also some very large particles around $1000 \mathrm{~nm}$ and a significant amount of smaller particles of approximately $200 \mathrm{~nm}$ or smaller (e and f). 


\section{Estimated deposited dose in lungs}

The estimated deposited dose in the lung and head was similar for $\mathrm{CeO}_{2}, 27 \% \mathrm{Zr}$-doped $\mathrm{CeO}_{2}$ and $78 \% \mathrm{Zr}$-doped $\mathrm{CeO}_{2} \mathrm{NPs}$, ranging from 618 to $639 \mathrm{ng}$ (approximately $60 \%$ of the inhaled dose). The deposition pattern in the different regions of the lungs was also similar for the different types of $\mathrm{CeO}_{2} \mathrm{NPs}$, with approximately $8 \%$ of the dose reaching the alveoli (see Table 3).

Table 3: Estimated deposition of inhaled $\mathrm{CeO}_{2}, 27 \% \mathrm{Zr}$-doped $\mathrm{CeO}_{2}$ and $78 \% \mathrm{Zr}$-doped $\mathrm{CeO}_{2} \mathrm{NPs}$ in different regions of the lungs.

\begin{tabular}{|c|c|c|c|c|c|c|}
\hline & \multirow{2}{*}{$\begin{array}{l}\text { Total } \\
\text { inhaled } \\
\text { dose }(\mu \mathrm{g})^{*}\end{array}$} & \multirow{2}{*}{$\begin{array}{l}\text { Total } \\
\text { deposited } \\
\text { dose }(\mu g)^{* *}\end{array}$} & \multicolumn{3}{|c|}{$\begin{array}{l}\text { Deposited fraction per region } \\
\text { (\% of inhaled dose) } * *\end{array}$} & \multirow{2}{*}{$\begin{array}{l}\text { Retained } \\
\text { dose in lung } \\
(\mu \mathrm{g})^{* * *}\end{array}$} \\
\hline & & & $\begin{array}{c}\text { Head } \\
(\%)\end{array}$ & $\begin{array}{l}\text { Tracheobronchial } \\
\text { region (\%) }\end{array}$ & $\begin{array}{l}\text { Alveolar } \\
\text { region (\%) }\end{array}$ & \\
\hline $\mathrm{CeO}_{2} \mathrm{NPs}$ & 1027 & 618 & 48.8 & 3.5 & 8.0 & 16.2 \\
\hline $27 \% \mathrm{Zr}$-doped $\mathrm{CeO}_{2} \mathrm{NPs}$ & 1043 & 625 & 48.8 & 3.4 & 7.9 & 16.2 \\
\hline $75 \%$ Zr-doped $\mathrm{CeO}_{2} \mathrm{NPs}$ & 1056 & 639 & 49.8 & 3.3 & 7.5 & 15.6 \\
\hline
\end{tabular}

* Total amount inhaled $=$ estimated as tidal volume $(0.203 \mathrm{~mL}) \times$ breathing frequency $(353 \mathrm{~min}-1) \times$ exposure concentration $\left(\approx 4 \times 10^{-3} \mathrm{\mu g} / \mathrm{mL}\right.$ ) $\times$ exposure duration (3600 $\left.\mathrm{min}\right)$.

** $\quad$ Estimated using the MPPD model

*** Retained dose in the tracheobronchial and alveolar region 4 weeks post-exposure, estimated using the MPPD model.

\section{Quantification of Ce and Zr in tissues}

The highest concentrations of $\mathrm{Ce}$ and $\mathrm{Zr}$ were found in the lung, followed by much lower concentrations in the heart, spleen, kidneys and liver, respectively (Figure 7). As would be expected, significantly higher levels of $\mathrm{Ce}$ and $\mathrm{Zr}$ were observed in the lungs of exposed mice compared to the controls. Increasing amounts of Zr-doping in the NP exposure did not result in decreasing Ce concentrations or increasing $\mathrm{Zr}$ concentrations in the lung. In most of the other organs, the $\mathrm{Ce}$ and $\mathrm{Zr}$ concentrations were not statistically significantly different from the background concentrations measured in the controls. A few exceptions were observed, for example, the Ce concentration in the hearts of mice exposed to $78 \% \mathrm{Zr}$-doped $\mathrm{CeO}_{2}$.

\section{Hematology}

ApoE ${ }^{-1}$ mice had more neutrophils compared to exposed and control C57BL/6J and 5xFAD mice ( $p<0.05$ in Tukey's post-hoc test following one-way ANOVA). No statistically significant differences were observed in the total white blood cell counts (data not shown) or differential white blood cell counts (Figure 8) of the exposed groups compared to the controls in blood from all strains of mice, except for a small decrease in neutrophils in $\mathrm{ApoE}^{-\%}$ mice exposed to 78\%-doped $\mathrm{CeO}_{2}$ NPs (Tukey's post-hoc test following one-way ANOVA with $p=0.02$ ). In addition, there was a decrease (post-hoc test for linear trend following one-way ANOVA with $\mathrm{p}=0.002$ ) in neutrophils in $\mathrm{ApoE}^{-/}$mice with increasing amounts of $\mathrm{Zr}$-doping. 


\section{Ce and $\mathrm{Zr}$ concentrations in different organs}

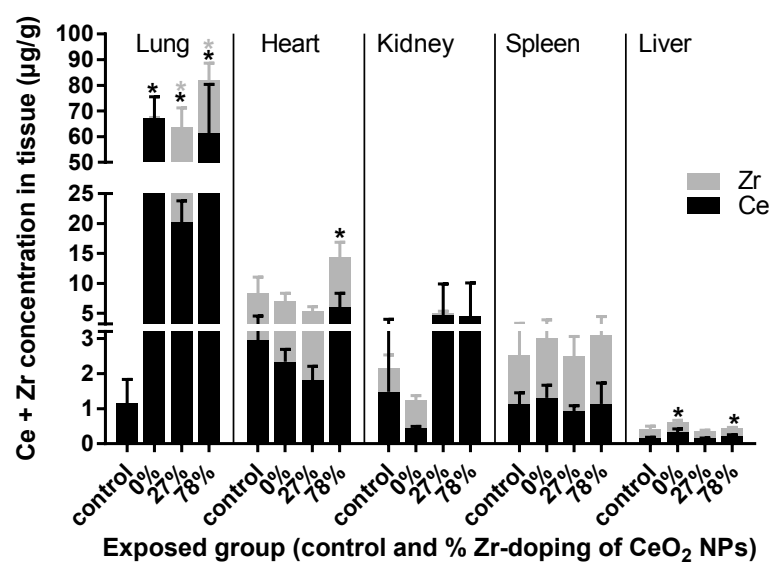

Figure7: Ce and $\mathrm{Zr}$ concentrations in organs 4 weeks after exposure to clean air (control) or $0 \%, 27 \%$ or $78 \% \mathrm{Zr}$ doped $\mathrm{CeO}_{2} \mathrm{NPs}$ (exposed). Mean $\pm \mathrm{SD}, \mathrm{n}=4-6,{ }^{*}=\mathrm{Ce}$ or $\mathrm{Zr}$ concentration is statistical significantly different from the control in Tukey's post-hoc test following one-way ANOVA with $p<0.05$. The bar graphs are stacked and represent the sum of the Ce concentration (bottom black colored part of each bar) and Zr concentration (upper gray colored part of each bar) in the different organs. With a few exceptions (e.g. the lung), the Ce and Zr concentrations of the exposed animals were not statistical significantly different from the control animals in most organs. The $\mathrm{Zr}$ concentration in the lungs of the control group and in the kidneys of the group exposed to $78 \% \mathrm{Zr}$-doped $\mathrm{CeO}_{2} \mathrm{NPs}$ was below the detection limit.

\section{Differential white blood cell count}

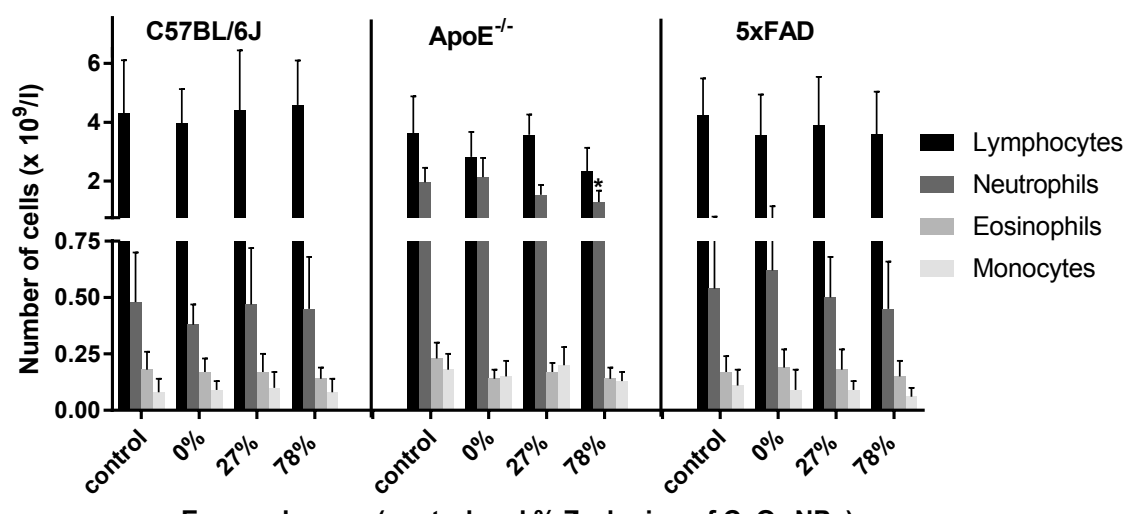

Exposed group (control and \% $\mathrm{Zr}$-doping of $\mathrm{CeO}_{2} \mathrm{NPs}$ )

Figure 8: Differential white blood cell count 4 weeks after exposure to clean air (control) or $0 \%, 27 \%$ or $78 \% \mathrm{Zr}$ doped $\mathrm{CeO}_{2} \mathrm{NPs}$ (exposed). Mean $\pm \mathrm{SD}, \mathrm{n}=8-16,{ }^{*}=$ statistical significantly different from the control in Tukey's posthoc test following one-way ANOVA with $p<0.05$. No statistically significant differences between the different types of white blood cells were observed between control and exposed animals, except for the neutrophils in ApoE ${ }^{-1}$ mice exposed to $78 \% \mathrm{Zr}$-doped $\mathrm{CeO}_{2} \mathrm{NPs}$. The number of basophils and large unstained cells were very small and are thus not depicted. 


\section{Bronchoalveolar lavage}

The number of lymphocytes in the control C57BL/6J mice was relatively high (7.7\%), but this was due to an outlier and did not represent an inflammatory response. No statistically significant differences were observed in the total cell counts (data not shown) or differential cell counts in BALF of the exposed groups compared to the controls for any strain of mouse (Figure 9), except for the total cell count in 5xFAD mice exposed to $27 \% \mathrm{Zr}$-doped $\mathrm{CeO}_{2} \mathrm{NPs}$, which was lower than that of the control 5xFAD mice. There was a decrease $(p<0.1$; post-hoc test for linear trend following one-way ANOVA) in total cell counts ( $p=0.075), \%$ macrophages $(p=0.014)$, and \% neutrophils $(p=0.05)$ in $\mathrm{ApoE}^{-/-}$mice with increasing amounts of $\mathrm{Zr}$-doping. However, no such trends were observed in C57BL/6J or 5xFAD mice. Similarly, no statistically significant differences were observed for LDH, ALP or GGT protein levels between the exposed and control groups (Figure 10). No constitutive differences in differential cell counts or protein levels were observed between the different mice strains.

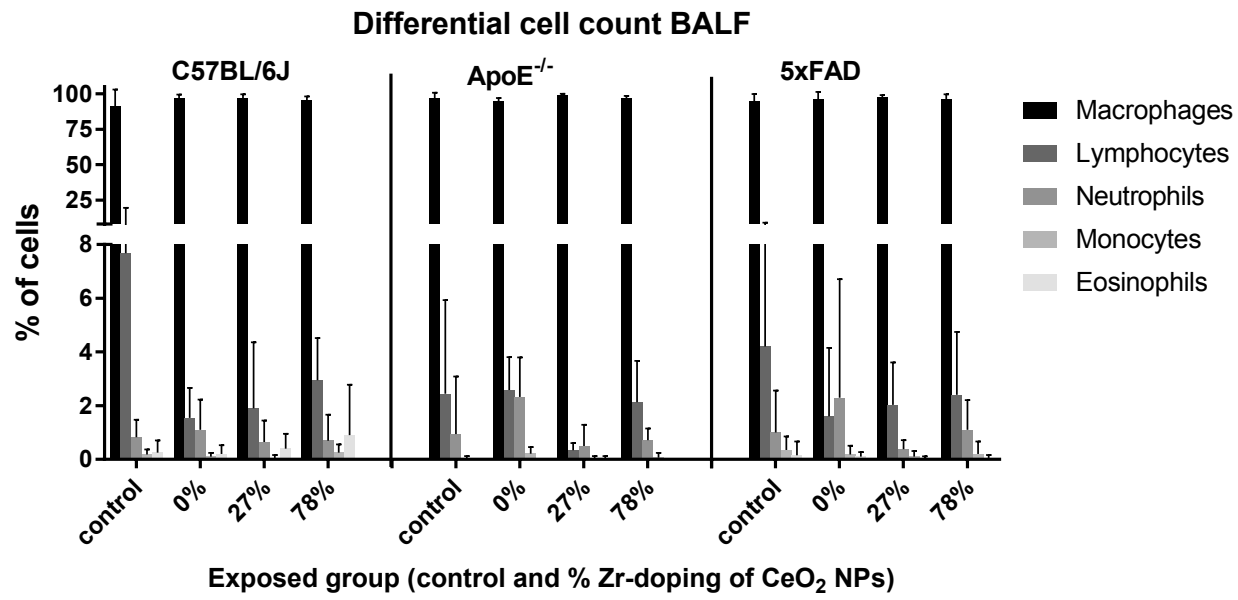

Figure 9: Differential cell count in bronchoalveolar lavage fluid (BALF) 4 weeks after exposure to clean air (control) or $\mathrm{CeO}_{2}, 27 \% \mathrm{Zr}$-doped $\mathrm{CeO}_{2}$ or $78 \% \mathrm{Zr}$-doped $\mathrm{CeO}_{2} \mathrm{NPs}$ (exposed). Mean $\pm \mathrm{SD}, \mathrm{n}=5-16$, * =statistical significantly different from the control in Tukey's post-hoc test following one-way ANOVA with $p<0.05$. No statistically significant differences in the different types of cells were observed between control and exposed animals, except for the number of macrophages in 5xFAD mice exposed to $27 \% \mathrm{Zr}$-doped $\mathrm{CeO}_{2} \mathrm{NPs}$. 


\section{Proteins in BALF}

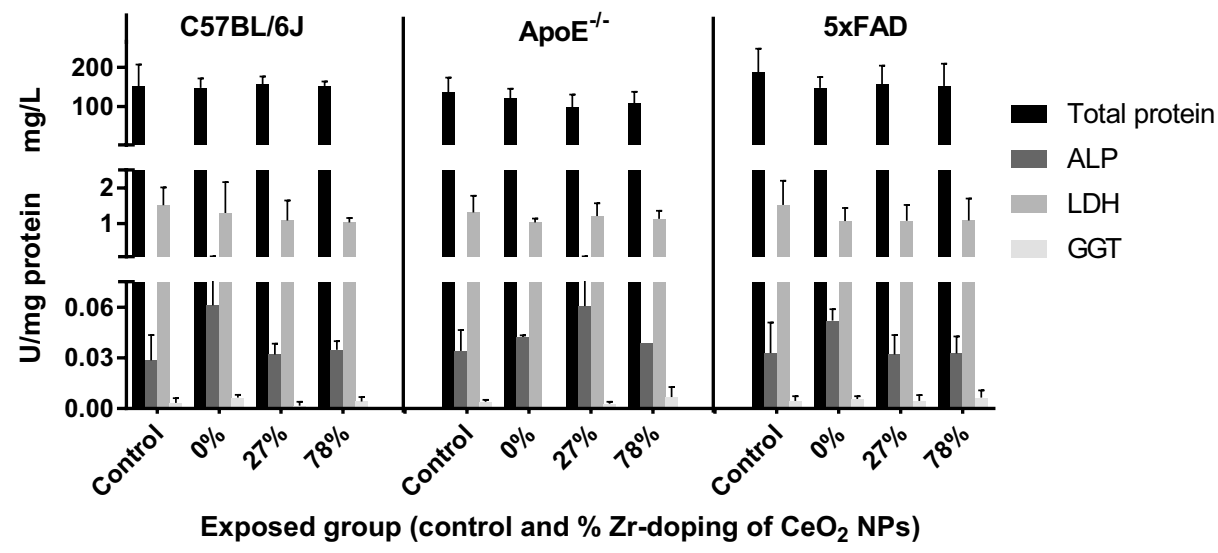

Figure 10: Total protein, Lactate Dehydrogenase (LDH), Alkaline Phosphatase (ALP) and Gamma-Glutamyl Transpeptidase (GGT) levels in bronchoalveolar lavage fluid (BALF) 4 weeks after exposure to clean air (control) or $\mathrm{CeO}_{2}$ and $27 \% \mathrm{Zr}$-doped $\mathrm{CeO}_{2}$ or $78 \% \mathrm{Zr}$-doped $\mathrm{CeO}_{2} \mathrm{NPs}$ (exposed). Mean $\pm \mathrm{SD}, \mathrm{n}=5-16$. No statistically significant differences in the total protein, LDH, ALP or GGT were observed between control and exposed animals (Tukey's post-hoc test following one-way ANOVA with $\mathrm{p}<0.05$ ). 


\section{Histopathology}

\section{Lung}

While modest, an increased incidence in minimal chronic bronchoalveolar or alveolar inflammation was observed in the exposed animals compared to the control mice (see Figure $11 a, b$ and $c$ ). The increase did not appear to be related to the percentage $\mathrm{Zr}$-doping (Table 4).

Table 4: Histopathological findings in lung of $\mathrm{C} 57 \mathrm{BL} / 6 \mathrm{~J}, \mathrm{ApoE}^{-/}$and $5 \times \mathrm{FAD}$ mice.

\begin{tabular}{|c|c|c|c|c|c|c|}
\hline \multicolumn{2}{|c|}{ Histopathological finding $\rightarrow$} & \multicolumn{2}{|c|}{$\begin{array}{l}\text { Chronic bronchoalveolar or } \\
\text { alveolar inflammation }\end{array}$} & \multirow{2}{*}{$\begin{array}{l}\begin{array}{l}\text { Alveolar } \\
\text { macrophages }\end{array} \\
\text { Minimal }\end{array}$} & \multicolumn{2}{|c|}{$\begin{array}{l}\text { Particle loaded } \\
\text { alveolar macrophages }\end{array}$} \\
\hline Strain $\downarrow$ & Treatment $\downarrow$ & Minimal & Slight & & Minimal & Slight \\
\hline \multirow[t]{5}{*}{ C57BL/6J } & Control & $60 \%(3 / 5)^{a}$ & - & - & - & - \\
\hline & Total exposed & $67 \%(10 / 15)$ & - & $13 \%(2 / 15)$ & - & - \\
\hline & $\mathrm{CeO}_{2}$ & $80 \%(4 / 5)$ & - & $20 \%(1 / 5)$ & - & - \\
\hline & $27 \% \mathrm{Zr}$-doped $\mathrm{CeO}_{2}$ & $40 \%(2 / 5)$ & - & $20 \%(1 / 5)$ & - & - \\
\hline & $78 \% \mathrm{Zr}$-doped $\mathrm{CeO}_{2}$ & $80 \%(4 / 5)$ & - & - & - & - \\
\hline \multirow[t]{5}{*}{$\mathrm{ApoE}^{-/}$} & Control & $25 \%(2 / 8)$ & - & - & - & - \\
\hline & Total exposed & $42 \%(10 / 24)$ & - & - & $4 \%(1 / 24)$ & $25 \%(6 / 24)$ \\
\hline & $\mathrm{CeO}_{2}$ & $38 \%(3 / 8)$ & - & - & $13 \%(1 / 8)$ & $75 \%(6 / 8)$ \\
\hline & $27 \% \mathrm{Zr}$-doped $\mathrm{CeO}_{2}$ & $38 \%(3 / 8)$ & $13 \%(1 / 8)$ & - & - & - \\
\hline & $78 \% \mathrm{Zr}$-doped $\mathrm{CeO}_{2}$ & $50 \%(4 / 8)$ & - & - & - & - \\
\hline \multirow[t]{5}{*}{$5 x F A D$} & Control & $69 \%(11 / 16)$ & - & $6 \%(1 / 16)$ & - & - \\
\hline & Total exposed & $72 \%(13 / 18)$ & - & $17 \%(3 / 18)$ & - & - \\
\hline & $\mathrm{CeO}_{2}$ & $83 \%(5 / 6)$ & - & $17 \%(1 / 6)$ & - & - \\
\hline & $27 \% \mathrm{Zr}$-doped $\mathrm{CeO}_{2}$ & $67 \%(4 / 6)$ & - & - & - & - \\
\hline & $78 \% \mathrm{Zr}$-doped $\mathrm{CeO}_{2}$ & $67 \%(4 / 6)$ & - & $33 \%(2 / 6)$ & - & - \\
\hline
\end{tabular}

a Percentage of animals affected. Within brackets the number of animals with histopathological findings versus number of animals evaluated. 


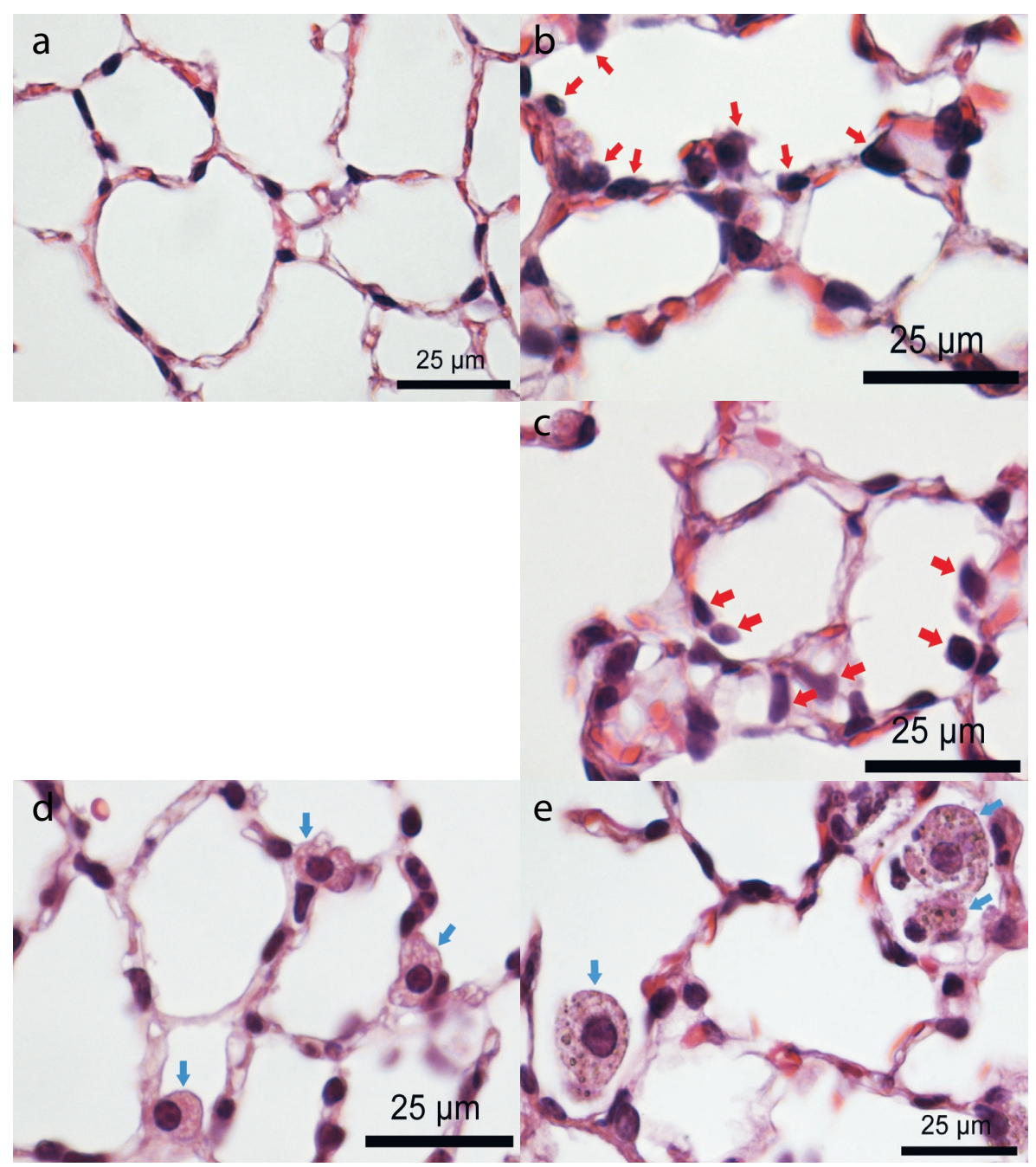

Figure 11: Lung sections showing minimal chronic bronchoalveoloar inflammation, alveolar inflammation and particle loaded alveolar macrophages in mice exposed to doped and undoped $\mathrm{CeO}_{2} \mathrm{NP}$ exposure. Lung sections of C57BL/6J mice exposed to clean air (a) and 78\% Zr-doped $\mathrm{CeO}_{2} \mathrm{NPs}$ (b and c) and of ApoE-/- mice exposed to 27\% $\mathrm{Zr}$-doped $\mathrm{CeO}_{2} \mathrm{NPs}(\mathrm{d})$ and undoped $\mathrm{CeO}_{2} \mathrm{NPs}(\mathrm{e})$. Red arrows indicate mononuclear inflammatory cells on the septa of the alveoli, indicating minimal chronic bronchoalveolar inflammation (b) or inside the alveoli, indicating minimal alveolar inflammation (c). Lung sections of mice with chronic and alveolar inflammation were similar across the different mouse models and experimental groups. Blue arrows indicate empty (d) or particle loaded (e) alveolar macrophages.

Particle loaded alveolar macrophages (Figure $11 \mathrm{e}$ ) were observed in seven of the eight ApoE $/$ mice exposed to undoped $\mathrm{CeO}_{2}$ NPs. This effect was not observed in ApoE $\mathrm{F}^{-}$mice exposed to $27 \%$ or $78 \% \mathrm{Zr}$-doped $\mathrm{CeO}_{2} \mathrm{NPs}$ or any other exposure or the control group of the other mouse models (Table 4), indicating Zr-doping may influence particle loading of alveolar macrophages in ApoE-/- mice. In the bronchoalveolar lavage fluid particle loaded macrophages were seen in all the NP exposed animals but not in the control groups. 


\section{Cardiovascular Effects}

ApoE ${ }^{-/}$mice exhibited regions of dense plaques in the aortic arch and branch points of large arteries. Plaques were composed of fibroblastic matrix, smooth muscle cells, lipid cavities and cholesterol crystals (Figure 12a). Atherosclerotic burden was quantified in the brachiocephalic artery, with control (air-exposed) mice having a mean plaque size of $94 \pm$ $9 \%$ (standardized to the area of the vascular media). Exposure to undoped or $\mathrm{Zr}$-doped $\mathrm{CeO}_{2}$ NPs did not have a significant effect on the atherosclerotic burden (mean plaque size) of these arteries ( $p=0.62$; One-way ANOVA; Figure 12b). However, there was an increase (posthoc test for linear trend following one-way ANOVA with $p=0.09$ ) in the proportion of plaque staining positive for mac-2 (i.e. macrophage-derived foam cells; Figure 12c) with increasing Zr content of the $\mathrm{CeO}_{2}$ NP exposure (Figure 12d).

$\mathrm{a}$

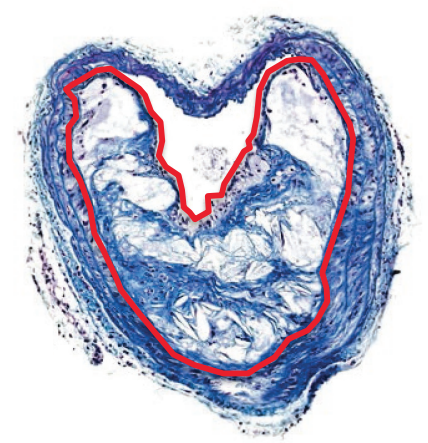

C

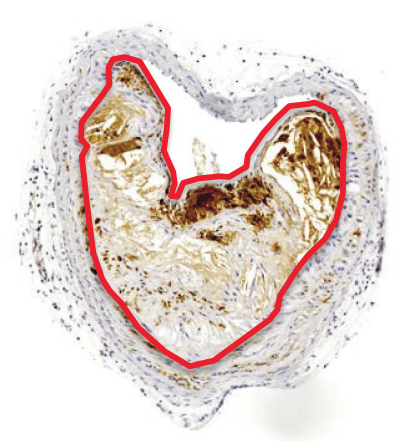

b

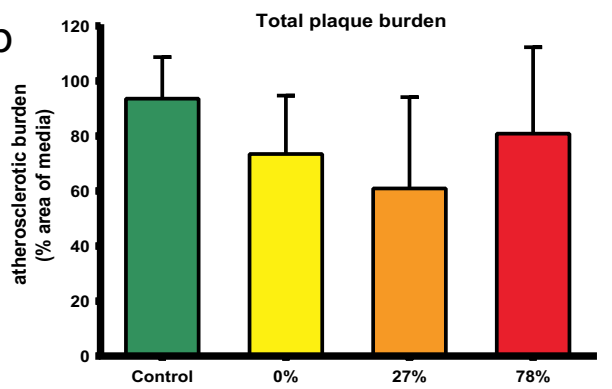

d

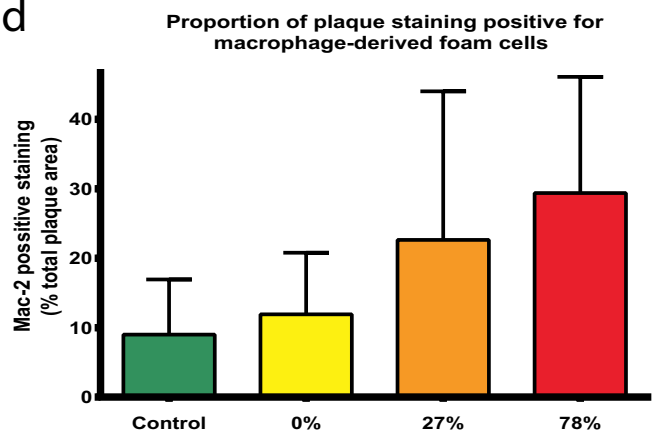

Figure 12: Effect of $\mathrm{Zr}$-doped $\mathrm{CeO}_{2} \mathrm{NPs}$ on atherosclerosis in the brachiocephalic artery of ApoE ${ }^{-1}$ mice after inhalation. (a) Representative section of brachiocephalic artery showing a large plaque on the intimal surface of the artery. The red outline highlights the area of the plaque. (b) Quantification of atherosclerotic burden throughout the brachiocephalic artery. Mean \pm SEM ( $n=3-7)$. Undoped ( $0 \%)$ or $\mathrm{Zr}$-doped ( $27 \%$ and $78 \%$ ) $\mathrm{CeO}_{2} \mathrm{NP}$ exposure did not significantly affect plaque size compared to clean air exposure (controls). (c) Immunohistochemical staining for mac-2 (brown stain, showing macrophage-derived foam cells). The red outline highlights the area of the plaque. (d) Proportion of plaque staining positive for mac-2 following exposure to clean air (control), undoped $(0 \%)$ and Zr-doped (27\% and 78\%) $\mathrm{CeO}_{2}$ NPs. Mean \pm SEM $(n=4-7)$. No statistically significant differences were observed between control and exposed animals (Tukey's post-hoc test, following one-way ANOVA with $p<0.05$ ). 


\section{Other organs}

No treatment related effects on organ weights or macroscopic findings (data not shown) were observed and thus no histopathological analysis was performed on the other organs.

\section{Discussion}

In this subacute inhalation study, only modest biological effects were observed after exposure to $\mathrm{CeO}_{2} \mathrm{NPs}$ or $\mathrm{Zr}$-doped $\mathrm{CeO}_{2} \mathrm{NPs}$ in healthy mice and mouse models of atherosclerosis and Alzheimer's disease. Accordingly, altering the redox activity of nanomaterials by Zr-doping had a limited impact on the bioactivity, in the absence of any overt toxicity of the $\mathrm{CeO}_{2}$ parent material.

\section{Zr-doping of $\mathrm{CeO}_{2} \mathrm{NPS}$}

Zr-doping of $\mathrm{CeO}_{2} \mathrm{NPs}$ was expected to increase the antioxidant potential of $\mathrm{CeO}_{2} \mathrm{NPS}$ by shifting the Ce valence state towards $3^{+}$, increasing its capacity to shift from $3^{+}$to $4^{+}$ and thus increasing its antioxidant potential and thereby reducing adverse pulmonary and cardiovascular effects. Our UV-vis analyses confirmed that Zr-doping does shift the valence state towards $3^{+}$(see Figure 2 ). Furthermore, ESR analysis using a DMPO spin trap in combination with $\mathrm{H}_{2} \mathrm{O}_{2}$ showed that high concentrations of $\mathrm{Zr}$-doping increased the scavenging capacity for ROS by $\mathrm{CeO}_{2} \mathrm{NPs}$ in a cell-free system (see Figure 3). In addition, in vitro tests indicated that $\mathrm{Zr}$-doping decreased the cytotoxicity of $\mathrm{CeO}_{2} \mathrm{NPs}$ in human alveolar cells (A549) (see Figure 4). However, in vivo Zr-doping had a limited impact on the pulmonary and cardiovascular effects.

Previous studies investigated the effect of samarium (Sm) or gadolinium (Gd) doping of $\mathrm{CeO}_{2}$ NPs (Celardo et al., 2011, Dunnick et al., 2015, Dunnick et al., 2016). Sm-doping decreased the $\mathrm{Ce}^{3+} / \mathrm{Ce}^{4+}$ ratio and thereby the ability to shift from $3^{+}$to $4^{+}$, decreasing the antioxidant properties of $\mathrm{CeO}_{2}$ NPs in leukocyte (U937 and Jurkat) cell lines (Celardo et al., 2011). Gddoping increased the $\mathrm{Ce}^{3+} / \mathrm{Ce}^{4+}$ ratio, but also decreased the ability to shift from $3^{+}$to $4^{+}$and the antioxidant properties, suggesting that differences in reactivity of $\mathrm{CeO}_{2} \mathrm{NPs}$ are due to the ability of $\mathrm{Ce}$ to transition between the two valence states rather than dependent on a specific valence state (Dunnick et al., 2015, Dunnick et al., 2016). Although Zr-doping increased the $\mathrm{Ce}^{3+} / \mathrm{Ce}^{4+}$ ratio of the $\mathrm{CeO}_{2} \mathrm{NPs}$, the ability to shift from $3^{+}$to $4^{+}$may be reduced instead of enhanced due to $\mathrm{Zr}$-doping, because the $\mathrm{Zr}$-atoms occupy some of the $4^{+}$sites.

In addition, the antioxidant activity of $\mathrm{CeO}_{2} \mathrm{NPs}$ can also be affected by the external environment, especially the anions (Xue et al., 2012). Xue et al. (2012) showed that $\mathrm{CeO}_{2}$ NPs scavenge hydroxyl radicals $(\cdot \mathrm{OH})$ in Tris hydrochloride (Tris- $\mathrm{HCl}$ ) and sulfate systems, but lose their scavenging capacity in PBS. Their explanation for this is that the antioxidant activity of $\mathrm{CeO}_{2} \mathrm{NPs}$ is not only determined by its ability to convert $\mathrm{Ce}^{3+}$ into $\mathrm{Ce}^{4+}$, but also 
by regeneration of $\mathrm{Ce}^{3+}$ by reduction of $\mathrm{Ce}^{4+}$ on the surface of the nanoparticles. According to Xue et al. (2012) in PBS cerium phosphate is formed on the surface of the NPs, which prevents the regeneration of $\mathrm{Ce}^{3+}$ from $\mathrm{Ce}^{4+}$ during the redox cycling preventing the $\mathrm{CeO}_{2}$ $\mathrm{NPs}$ to scavenge $\cdot \mathrm{OH}$. Although the aerosols of the $\mathrm{Zr}$-doped $\mathrm{CeO}_{2} \mathrm{NPs}$ were prepared using ultrapure water instead of PBS, the antioxidant potential of the $\mathrm{CeO}_{2} \mathrm{NPs}$ may be similarly altered once deposited in the lungs of the mice.

\section{Particle deposition and distribution to other organs}

The deposition along different regions of the respiratory tract of mice, calculated with the MPPD model (v3.04), was similar for the different types of undoped and $\mathrm{Zr}$-doped $\mathrm{CeO}_{2} \mathrm{NPS}_{\text {. }}$ In all cases, the estimated fraction of particle deposition in the lung (tracheobronchial and alveolar region) was approximately $11 \%$ of the inhaled mass dose.

As expected, the highest cerium ( $\mathrm{Ce}$ ) and zirconium ( $\mathrm{Zr}$ ) concentrations, as measured by ICP-MS analysis of tissues, were found in the lung. The MPPD model can be used to predict the Ce dose in the lung 4 weeks post-exposure if it is assumed there is an even distribution of the $\mathrm{CeO}_{2}$ NPs in the lungs. Based on the estimated retained dose of $\mathrm{CeO}_{2} \mathrm{NPs}$ (with $0 \% \mathrm{Zr}$-doping) in the lung (16.2 $\mu \mathrm{g}$, see Table 3) and the average lung weight (158 $\mathrm{mg})$, the expected Ce concentration in the lung is approximately $103 \mu \mathrm{g} / \mathrm{g}$. The measured Ce concentration in the right lungs of the undoped $\mathrm{CeO}_{2} \mathrm{NP}$ exposed mice was slightly lower $(67 \mu \mathrm{g} / \mathrm{g})$ but in the same order of magnitude. The difference between the predicted and measured concentrations may reflect the lung clearance rate used by the MPPD model compared to the actual lung clearance rate in vivo.

Background levels of $\mathrm{Ce}$ and $\mathrm{Zr}$ were detected in various organs of animals exposed to clean air (controls). This might be caused by $\mathrm{Ce}$ and $\mathrm{Zr}$ contamination of the drinking water, food and/or bedding of the animals. Although $\mathrm{Ce}$ and $\mathrm{Zr}$ concentrations are not routinely measured in drinking water, food or bedding, Ce was detected in rat chow in a concentration of $0.22 \mathrm{mg} \mathrm{Ce} / \mathrm{kg}$ food (Yokel et al., 2012) and in tap water in concentrations of $\sim 0.0009$ $\mathrm{ug} / \mathrm{L}$ in the US (Donovan et al., 2016) and $0.018-0.084 \mathrm{\mu g} / \mathrm{L}$ in Croatia (Fiket et al., 2015). In addition, Ce has been detected in various organs of control animals in previous in vivo studies (Yokel et al., 2012, Yokel et al., 2013).

The $\mathrm{Ce}$ and $\mathrm{Zr}$ concentrations in the lung did not reflect the different percentages of $\mathrm{Zr}$ doping of the NPs, as would have been expected. Increasing amounts of Zr-doping did not lead to higher Zr concentrations or lower Ce concentrations. Moreover, surprisingly low Ce concentrations were found in the lungs of mice exposed to $27 \% \mathrm{Zr}$-doped $\mathrm{CeO}_{2} \mathrm{NPS}$ compared to mice exposed to $78 \% \mathrm{Zr}$-doped $\mathrm{CeO}_{2}$. These findings are unlikely to be due to the differences in exposure or deposition of the NPs, since the gravimetric mass concentrations and size distribution of the aerosols were almost identical (Table 2). These unexpected 
findings might reflect differences in clearance, de-agglomeration or dissolution of the different $\mathrm{CeO}_{2}$ NPs types in the lung, based on, for example, differences in agglomeration size and surface specific uptake by macrophages. We speculate that $\mathrm{Zr}$-doping may increase the clearance of the NPs, since a previous study showed that the decrease in lung burden during the post-exposure period of 21 days was markedly higher for $\mathrm{ZrO}_{2}$ NPs $(42-75 \%$ decrease) compared to $\mathrm{CeO}_{2}$ NPs (5-7\% decrease) (Landsiedel et al., 2014). Alternatively, it is possible that the particles may have had different effective densities that might have affected the distribution pattern.

The concentrations of $\mathrm{Ce}$ and $\mathrm{Zr}$ in heart, spleen, kidneys and liver were much lower than what was measured in the lung. Similar distribution patterns have been found in other studies after inhalation of $\mathrm{CeO}_{2}$ NPs (Geraets et al., 2012, Aalapati et al., 2014, Keller et al., 2014).

\section{Pulmonary effects}

$\mathrm{CeO}_{2}$ NP exposure had no statistically significant effects on total and differential cell counts, LDH, ALP, GGT or total protein levels in the BALF of any of the tested mouse strains 4 weeks post-exposure, irrespective the $\mathrm{Zr}$ content. Only modest inflammatory lesions were found by histopathological analysis of lung sections of $\mathrm{CeO}_{2} \mathrm{NPs}$ exposed mice. As these findings were not related to the amount of Zr-doping, the observed pulmonary effects are likely to be caused by the physical interaction between the alveolar surface and the large reactive surface area of the particles, an observation not uncommon with poorly-soluble nanoparticles (Greim and Ziegler-Skylakakis, 2007). One exception to these findings were the particle-loaded alveolar macrophages in histological lung sections of $\mathrm{ApoE}^{-/}$mice that were only observed after exposure to $\mathrm{CeO}_{2} \mathrm{NPs}$ without Zr-doping , indicating Zr-doping may influence the occurrence of particle loaded alveolar macrophages. Interestingly, in the BALF, particle-loaded macrophages were seen in all the exposed groups (all mouse models and all $\mathrm{CeO}_{2} \mathrm{NP}$ types). This observation might reflect differences in the dynamics of macrophages of the $\mathrm{ApoE}^{-/-}$mice; for example the inherently higher number of macrophages in the lungs of this strain of mouse (Grainger et al., 2004), which can be further increased by a high fat diet (Naura et al., 2009). Differences in the ability of the undoped and the $\mathrm{Zr}$-doped $\mathrm{CeO}_{2}$ NP types to influence the NP uptake, damage and turnover of macrophages in the lung may result in differences in the release of the NPs from macrophages in the lung, resulting in the observed $\mathrm{Zr}$-doping related differences in the occurrence of particle-loaded alveolar macrophages in the histological lung sections 4 weeks post-exposure.

Other inhalation studies have reported more prominent effects of $\mathrm{CeO}_{2} \mathrm{NPs}$ than those observed in our study, including increased neutrophils and lymphocytes in BALF, increased concentrations of biochemical markers of inflammation in BALF (LDH, ALP, GGT, N-acetyl beta-d-glucosaminidase (NAG) and cytokine-induced neutrophil chemoattractant-1 
(CINC-1)), increased lung weight, inflammatory lung lesions (e.g. severe alveolitis, particle loaded macrophages and granulomas, lung fibrosis) and accumulation of particle-loaded macrophages in lymph nodes and liver (Srinivas et al., 2011, Demokritou et al., 2013, Aalapati et al., 2014, Gosens et al., 2014, Keller et al., 2014) (See Tables S1). It is notable that most of these studies were performed in rats, which tend to be more susceptible to pulmonary inflammation induced by poorly soluble particles than mice (Borm et al., 2015). Some of the effects observed in previous studies only occurred at higher exposure concentrations (e.g. $5,10,25$ or $50 \mathrm{mg} / \mathrm{m}^{3}$ for $6 \mathrm{~h} /$ day, $5 \mathrm{~d} / \mathrm{w}$ for 4 weeks), compared to the exposure of $4 \mathrm{mg} / \mathrm{m}^{3}$ for $3 \mathrm{~h} / \mathrm{day}, 5 \mathrm{~d} / \mathrm{w}$ for 4 weeks we used in our study. Additionally, our study focused on the presence of persistent toxicity 4 weeks post-exposure, when inflammation induced directly after exposure will have gradually diminished to a level that does not manifest in the BALF or histopathology. The prolonged recovery time in our study provides an opportunity to assess whether the biological effects lead to persistent adverse outcomes. Nevertheless, we acknowledge that several prior studies have demonstrated long-lasting pulmonary effects, including raised neutrophils and lymphocytes in BALF and accumulation of particle-loaded macrophages and granulomas in the lung (Aalapati et al., 2014, Keller et al., 2014, Morimoto et al., 2015). A 4-week inhalation exposure to similar levels of $\mathrm{CeO}_{2} \mathrm{NPs}$ in CD-1 mice caused inflammation-induced responses in the lung characterized by necrosis, fibrosis and granulomas 4 weeks post- exposure (Aalapati et al., 2014). Differences in the $\mathrm{CeO}_{2} \mathrm{NPs}$ and exposure characteristics could explain these diverging results. In our study, $\mathrm{CeO}_{2} \mathrm{NPs}$ were produced by hydrothermal synthesis, with primary particle size of $\sim 4.7 \mathrm{~nm}$, and aerosols with a mass median diameter (MMD) of $280 \mathrm{~nm}$ with a geometric standard deviation (GSD) of 1.56. Aalapati et al. (2014) used $\mathrm{CeO}_{2}$ NPs produced via the sol-gel method, with a primary diameter of 15-30 nm and aerosols with a mass median aerodynamic diameter (MMAD) of $1.4 \mu \mathrm{m}$ (GSD 2.4). Both the cumulative external dose, (i.e. concentration $x$ exposure duration) and the dose rate were very similar for both studies, however, differences in particle size distribution will have led to different biological effective pulmonary doses. The measured Ce concentration in the lung 4 weeks post-exposure reported by Aalipati et al. was much higher (approximately $500 \mu \mathrm{g} / \mathrm{g}$ tissue compared to $67 \mu \mathrm{g} / \mathrm{g}$ in our study). The higher Ce concentration is likely to have contributed to the greater pulmonary toxicity in the Aalapati study. Other possible explanations are that $\mathrm{CeO}_{2} \mathrm{NPs}$ generated via sol-gel method have a different redox activity, surface structure or other properties that make them more toxic than $\mathrm{CeO}_{2}$ NPs generated by hydrothermal synthesis. Furthermore, the strain (CD-1 mice), gender (male) and/or age (7-8 weeks) of the mice used by Aalapati et al. might be more sensitive to the effects of particle inhalation than the strain ( $\mathrm{C} 57 \mathrm{BL} / 6 \mathrm{~J}$ mice), gender (female) and/or age (9 weeks) of the mice used in our study.

The modest inflammatory lung lesions observed in this study were not related to the amount of $\mathrm{Zr}$-doping of the $\mathrm{CeO}_{2} \mathrm{NPs}$, with the exception of the particle-loaded alveolar macrophages in histological lung sections of $\mathrm{ApoE}^{-/-}$mice that were only observed after 
exposure to $\mathrm{CeO}_{2} \mathrm{NPs}$ without Zr-doping. Recently, another in vivo study (Dunnick et al., 2016) investigated the influence of $\mathrm{Gd}$-doping of $\mathrm{CeO}_{2} \mathrm{NPs}$ after inhalation exposure. In this study, Sprague Dawley rats were exposed to 0.5 or $1.0 \mathrm{mg} / \mathrm{kg}$ pure $\mathrm{CeO}_{2} \mathrm{NPs}$ and 10 or $20 \%$ Gd-doped $\mathrm{CeO}_{2} \mathrm{NPs}$ via intratracheal instillation. Gd-doping was expected to increase the pulmonary toxicity, but no major effect of doping on the pulmonary toxicity was observed 1,7 and 84 days after exposure of $\mathrm{CeO}_{2}$ NPs in rats. Our study used a post-exposure period of 4 weeks and an exposure concentration that caused pronounced pulmonary toxicity in a previous study (Aalapati et al., 2014). However, because only minimal pulmonary effects were observed after exposure to the $\mathrm{CeO}_{2} \mathrm{NPs}$ without $\mathrm{Zr}$-doping in our study, most of the hypothesized ameliorative effects resulting from the $\mathrm{Zr}$-doping may not have been visible under the present conditions.

\section{Cardiovascular effects}

There was no significant effect of $\mathrm{CeO}_{2} \mathrm{NP}$ exposure, or of the $\mathrm{Zr}$-doped variants, on atherosclerotic burden. Previous studies using this mouse model showed that pulmonary exposure to nano-sized carbon black (Niwa et al., 2007), carbon nanotubes (Li et al., 2007, Cao et al., 2014), nickel nanoparticles (Kang et al., 2011) and nano-titanium dioxide (Mikkelsen et al., 2011, Chen et al., 2013) promoted the development of atherosclerosis. There is also a large body of literature showing that inhalation of ultrafine particles in air pollution promotes atherosclerosis in man (Kunzli et al., 2011) and animal models (Moller et al., 2011, Miller et al., 2013). Environmental nanoparticles have a very varied composition, however, the ability of chemicals and metals on the particle surface to generate reactive oxygen species is believed to be a key determinant of their ability to induce cardiovascular effects (Miller et al., 2012). Thus, we envisioned that inhalation of nanomaterials with different amounts of $\mathrm{Zr}$-doping would also induce varying degrees of cardiovascular dysfunction. The lack of effect of $\mathrm{Zr}$-doping of $\mathrm{CeO}_{2} \mathrm{NPs}$ may reflect the low reactivity of the parent compound, or potentially the small degree of deposition in the alveoli, leading to limited lung inflammation or particle translocation to the bloodstream. Interestingly, though, the composition of atherosclerotic plaques tended to be altered by subacute exposure to $\mathrm{CeO}_{2}$ NPs with increasing Zr content. In particular, there was an increase in the plaque content of macrophage-derived foam cells with an increased proportion of $\mathrm{Zr}$ in the $\mathrm{CeO}_{2} \mathrm{NPs}$. In man, plaques with a greater degree of inflammation are more instable and prone to rupture; the result of which can lead to thrombotic occlusion of the vessel causing a cardiovascular event e.g. heart attack or stroke (Williams et al., 2002). These findings suggest that alterations in chemical composition of $\mathrm{CeO}_{2}$ may have the potential to increase the susceptibility of plaques to rupture without necessarily increasing overall plaque size. At present, given the low biological activity of the parent material and the direction of the association between plaque macrophages and Ce:Zr ratio, it is not possible to state with any confidence that this is due to differences in redox activity. Nevertheless, this observation merits further investigation with other types of redox-modified nanomaterials in experiments with larger groups of $\mathrm{ApoE}^{-/-}$mice or extended periods of exposure. 


\section{Future directions}

The use of a systematic series of NPs of a single compound is desirable; however, further studies with more inherently toxic nanomaterials are required to fully address the role of redox activity. Because of the minimal pulmonary and cardiovascular effects observed with the parent material ( $\mathrm{CeO}_{2} \mathrm{NPs}$ without $\mathrm{Zr}$-doping), alteration of the redox activity of these $\mathrm{CeO}_{2} \mathrm{NPs}$ may have a limited capacity to subsequently alter toxicity. Metals and metal oxides that have a conduction band energy (Ec) level that overlaps with the cellular redox potential $(-4.12$ to $-4.84 \mathrm{eV})$ were shown to have the ability to induce oxygen radicals, oxidative stress, and inflammation in vitro and in vivo (Zhang et al., 2012). Ongoing work in our laboratories will investigate this hypothesis, using a newly synthesized series of metal doped nanoparticles with a conduction band energy that overlaps the cellular redox potential.

\section{Conclusions}

This study investigated the pulmonary and cardiovascular effects of redox modification of metal oxide nanoparticles using varying levels of metal doping of a structurally reproducible material. $\mathrm{CeO}_{2}$ NPs had minimal pulmonary and cardiovascular effects following subacute inhalation at 4 weeks post-exposure. $\mathrm{Zr}$-doping of $\mathrm{CeO}_{2} \mathrm{NPs}$ had limited effects on these responses, although indications that $\mathrm{Zr}$-doping could potentially alter particle loading of alveolar macrophages and increase the inflammatory cell content in atherosclerosis plaques merit further investigation. Future studies with other types of redox-modified nanomaterials of greater inherent toxicity and a wider range of redox activities are required to fully assess the influence of redox-modification on the toxicity of nanomaterials.

\section{Acknowledgements}

The authors would like to thank Paul H.B. Fokkens, Daan L.A.C. Leseman, Liset J.J. de la Fonteyne, Piet K. Beekhof, Christine M.R. Soputan, Hans J.C. Strootman, Jolanda Rigters, Ron F. Vlug, Karin M.P. van den Hurk, Julia Kolling, Geert B. van der Horst, Rory Verhagen, Dirk J. Elberts, Pieter W. Dissel and Angéla Gomersbach-de Ridder for their help during the conduction of the animal experiment and valuable technical assistance. In addition, Geert B. van de Horst is acknowledged for his contribution to the generation of the cytotoxicity in A549 cells and Joost Lensen of Will Research for the histopathology.

\section{Funding}

The work leading to these results has received funding from the European Union's Seventh Framework Programme for research, technology development and demonstration under grant agreement $n^{\circ} 310451$ (NanoMILE) and the Netherlands Food and Consumer Product Safety Authority (NVWA). 


\section{References}

Aalapati, S., Ganapathy, S., Manapuram, S., Anumolu, G. \& Prakya, B.M., 2014. Toxicity and bioaccumulation of inhaled cerium oxide nanoparticles in CD1 mice. Nanotoxicology, 8, 786-98.

Borm, P., Cassee, F.R. \& Oberdorster, G., 2015. Lung particle overload: old school -new insights? Part Fibre Toxicol, 12, 10.

Cabanas, A., Darr, J.A., E., L. \& Poliakoff, M., 2000. A continous and clean one-step synthesis of nanoparticulate $\mathrm{Ce}_{1-\mathrm{x}} \mathrm{Zr}_{\mathrm{x}} \mathrm{O}_{2}$ solid solutions in near-critical water. Chem Comm, 2000, 901-902.

Cao, Y., Jacobsen, N.R., Danielsen, P.H., Lenz, A.G., Stoeger, T., Loft, S., Wallin, H., Roursgaard, M., Mikkelsen, L. \& Moller, P., 2014. Vascular effects of multiwalled carbon nanotubes in dyslipidemic ApoE-/- mice and cultured endothelial cells. Toxicol Sci, 138, 104-16.

Cassee, F.R., Campbell, A., Boere, A.J., Mclean, S.G., Duffin, R., Krystek, P., Gosens, I. \& Miller, M.R., 2012. The biological effects of subacute inhalation of diesel exhaust following addition of cerium oxide nanoparticles in atherosclerosis-prone mice. Environ Res, 115, 1-10.

Cassee, F.R., Van Balen, E.C., Singh, C., Green, D., Muijser, H., Weinstein, J. \& Dreher, K., 2011. Exposure, health and ecological effects review of engineered nanoscale cerium and cerium oxide associated with its use as a fuel additive. Crit Rev Toxicol, 41, 213-29.

Celardo, I., De Nicola, M., Mandoli, C., Pedersen, J.Z., Traversa, E. \& Ghibelli, L., 2011. Ce(3)+ ions determine redox-dependent anti-apoptotic effect of cerium oxide nanoparticles. ACS Nano, 5 , 4537-49.

Chen, T., Hu, J., Chen, C., Pu, J., Cui, X. \& Jia, G., 2013. Cardiovascular effects of pulmonary exposure to titanium dioxide nanoparticles in ApoE knockout mice. J Nanosci Nanotechnol, 13, 3214-22.

Coleman, R., Hayek, T., Keidar, S. \& Aviram, M., 2006. A mouse model for human atherosclerosis: long-term histopathological study of lesion development in the aortic arch of apolipoprotein E-deficient (EO) mice. Acta Histochem, 108, 415-24.

Demokritou, P., Gass, S., Pyrgiotakis, G., Cohen, J.M., Goldsmith, W., Mckinney, W., Frazer, D., Ma, J., Schwegler-Berry, D., Brain, J. \& Castranova, V., 2013. An in vivo and in vitro toxicological characterisation of realistic nanoscale $\mathrm{CeO}(2)$ inhalation exposures. Nanotoxicology, 7, 1338-50.

Donovan, A.R., Adams, C.D., Ma, Y., Stephan, C., Eichholz, T. \& Shi, H., 2016. Detection of zinc oxide and cerium dioxide nanoparticles during drinking water treatment by rapid single particle ICP-MS methods. Anal Bioanal Chem, 408, 5137-45.

Dunnick, K.M., Morris, A.M., Badding, M.A., Barger, M., Stefaniak, A.B., Sabolsky, E.M. \& Leonard, S.S., 2016. Evaluation of the effect of valence state on cerium oxide nanoparticle toxicity following intratracheal instillation in rats. Nanotoxicology, 10, 992-1000.

Dunnick, K.M., Pillai, R., Pisane, K.L., Stefaniak, A.B., Sabolsky, E.M. \& Leonard, S.S., 2015. The Effect of Cerium Oxide Nanoparticle Valence State on Reactive Oxygen Species and Toxicity. Biol Trace Elem Res, 166, 96-107.

Eom, H.J. \& Choi, J., 2009. Oxidative stress of $\mathrm{CeO} 2$ nanoparticles via p38-Nrf-2 signaling pathway in human bronchial epithelial cell, Beas-2B. Toxicol Lett, 187, 77-83. 
Fiket, Z., Rozmaric, M., Krmpotic, M. \& Benedik, L., 2015. Levels of major and trace elements, including rare earth elements, and (2)(3)(8)U in Croatian tap waters. Environ Sci Pollut Res Int, 22, 6789-99.

Fu, P.P., Xia, Q., Hwang, H.M., Ray, P.C. \& Yu, H., 2014. Mechanisms of nanotoxicity: generation of reactive oxygen species. J Food Drug Anal, 22, 64-75.

Geraets, L., Oomen, A.G., Schroeter, J.D., Coleman, V.A. \& Cassee, F.R., 2012. Tissue distribution of inhaled micro- and nano-sized cerium oxide particles in rats: results from a 28-day exposure study. Toxicol Sci, 127, 463-73.

Gosens, I., Mathijssen, L.E., Bokkers, B.G., Muijser, H. \& Cassee, F.R., 2014. Comparative hazard identification of nano- and micro-sized cerium oxide particles based on 28-day inhalation studies in rats. Nanotoxicology, 8, 643-53.

Grainger, D.J., Reckless, J. \& Mckilligin, E., 2004. Apolipoprotein E modulates clearance of apoptotic bodies in vitro and in vivo, resulting in a systemic proinflammatory state in apolipoprotein E-deficient mice. J Immunol, 173, 6366-75.

Greim, H. \& Ziegler-Skylakakis, K., 2007. Risk assessment for biopersistent granular particles. Inhal Toxicol, 19 Suppl 1, 199-204.

He, W., Liu, Y., Wamer, W.G. \& Yin, J.J., 2014. Electron spin resonance spectroscopy for the study of nanomaterial-mediated generation of reactive oxygen species. J Food Drug Anal, 22, 49-63.

Hirst, S.M., Karakoti, A.S., Tyler, R.D., Sriranganathan, N., Seal, S. \& Reilly, C.M., 2009. Anti-inflammatory properties of cerium oxide nanoparticles. Small, 5, 2848-56.

Kang, G.S., Gillespie, P.A., Gunnison, A., Moreira, A.L., Tchou-Wong, K.M. \& Chen, L.C., 2011. Longterm inhalation exposure to nickel nanoparticles exacerbated atherosclerosis in a susceptible mouse model. Environ Health Perspect, 119, 176-81.

Keller, J., Wohlleben, W., Ma-Hock, L., Strauss, V., Groters, S., Kuttler, K., Wiench, K., Herden, C., Oberdorster, G., Van Ravenzwaay, B. \& Landsiedel, R., 2014. Time course of lung retention and toxicity of inhaled particles: short-term exposure to nano-Ceria. Arch Toxicol, 88, 2033-59.

Kunzli, N., Perez, L., Von Klot, S., Baldassarre, D., Bauer, M., Basagana, X., Breton, C., Dratva, J., Elosua, R., De Faire, U., Fuks, K., De Groot, E., Marrugat, J., Penell, J., Seissler, J., Peters, A. \& Hoffmann, B., 2011. Investigating air pollution and atherosclerosis in humans: concepts and outlook. Prog Cardiovasc Dis, 53, 334-43.

Landsiedel, R., Sauer, U.G., Ma-Hock, L., Schnekenburger, J. \& Wiemann, M., 2014. Pulmonary toxicity of nanomaterials: a critical comparison of published in vitro assays and in vivo inhalation or instillation studies. Nanomedicine (Lond), 9, 2557-85.

Leung, Y.H., Yung, M.M., Ng, A.M., Ma, A.P., Wong, S.W., Chan, C.M., Ng, Y.H., Djurisic, A.B., Guo, M., Wong, M.T., Leung, F.C., Chan, W.K., Leung, K.M. \& Lee, H.K., 2015. Toxicity of CeO2 nanoparticles - the effect of nanoparticle properties. J Photochem Photobiol B, 145, 48-59.

Li, Z., Hulderman, T., Salmen, R., Chapman, R., Leonard, S.S., Young, S.H., Shvedova, A., Luster, M.I. \& Simeonova, P.P., 2007. Cardiovascular effects of pulmonary exposure to single-wall carbon nanotubes. Environ Health Perspect, 115, 377-82.

Lin, W., Huang, Y.W., Zhou, X.D. \& Ma, Y., 2006. Toxicity of cerium oxide nanoparticles in human lung cancer cells. Int J Toxicol, 25, 451-7. 
Mikkelsen, L., Sheykhzade, M., Jensen, K.A., Saber, A.T., Jacobsen, N.R., Vogel, U., Wallin, H., Loft, S. \& Moller, P., 2011. Modest effect on plaque progression and vasodilatory function in atherosclerosisprone mice exposed to nanosized TiO(2). Part Fibre Toxicol, 8, 32.

Miller, M.R., Mclean, S.G., Duffin, R., Lawal, A.O., Araujo, J.A., Shaw, C.A., Mills, N.L., Donaldson, K., Newby, D.E. \& Hadoke, P.W., 2013. Diesel exhaust particulate increases the size and complexity of lesions in atherosclerotic mice. Part Fibre Toxicol, 10, 61.

Miller, M.R., Shaw, C.A. \& Langrish, J.P., 2012. From particles to patients: oxidative stress and the cardiovascular effects of air pollution. Future Cardiol, 8, 577-602.

Moller, P., Mikkelsen, L., Vesterdal, L.K., Folkmann, J.K., Forchhammer, L., Roursgaard, M., Danielsen, P.H. \& Loft, S., 2011. Hazard identification of particulate matter on vasomotor dysfunction and progression of atherosclerosis. Crit Rev Toxicol, 41, 339-68.

Morimoto, Y., Izumi, H., Yoshiura, Y., Tomonaga, T., Oyabu, T., Myojo, T., Kawai, K., Yatera, K., Shimada, M., Kubo, M., Yamamoto, K., Kitajima, S., Kuroda, E., Kawaguchi, K. \& Sasaki, T., 2015. Pulmonary toxicity of well-dispersed cerium oxide nanoparticles following intratracheal instillation and inhalation. J Nanopart Res, 17, 442.

Naura, A.S., Hans, C.P., Zerfaoui, M., Errami, Y., Ju, J., Kim, H., Matrougui, K., Kim, J.G. \& Boulares, A.H., 2009. High-fat diet induces lung remodeling in ApoE-deficient mice: an association with an increase in circulatory and lung inflammatory factors. Lab Invest, 89, 1243-51.

Niwa, Y., Hiura, Y., Murayama, T., Yokode, M. \& Iwai, N., 2007. Nano-sized carbon black exposure exacerbates atherosclerosis in LDL-receptor knockout mice. Circ J, 71, 1157-61.

Park, E.J., Choi, J., Park, Y.K. \& Park, K., 2008. Oxidative stress induced by cerium oxide nanoparticles in cultured BEAS-2B cells. Toxicology, 245, 90-100.

Pesic, M., Podolski-Renic, A., Stojkovic, S., Matovic, B., Zmejkoski, D., Kojic, V., Bogdanovic, G., Pavicevic, A., Mojovic, M., Savic, A., Milenkovic, I., Kalauzi, A. \& Radotic, K., 2015. Anti-cancer effects of cerium oxide nanoparticles and its intracellular redox activity. Chem Biol Interact, 232, 85-93.

Pisoschi, A.M. \& Pop, A., 2015. The role of antioxidants in the chemistry of oxidative stress: A review. Eur J Med Chem, 97, 55-74.

Slob, W., 2002. Dose-response modeling of continuous endpoints. Toxicol Sci, 66, 298-312.

Srinivas, A., Rao, P.J., Selvam, G., Murthy, P.B. \& Reddy, P.N., 2011. Acute inhalation toxicity of cerium oxide nanoparticles in rats. Toxicol Lett, 205, 105-15.

Tsai, Y.Y., Oca-Cossio, J., Lin, S.M., Woan, K., Yu, P.C. \& Sigmund, W., 2008. Reactive oxygen species scavenging properties of $\mathrm{ZrO} 2-\mathrm{CeO} 2$ solid solution nanoparticles. Nanomedicine (Lond), 3, 637 45.

Unfried, K.A., Catrin; Klotz, Lars-Oliver; Von Mikecz, Anna; Grether-Beck, Susanne; Schins, Roel P.F. , 2007. Cellular responses to nanoparticles: Target structures and mechanisms. Nanotoxicology, 1, 52-71.

Williams, H., Johnson, J.L., Carson, K.G. \& Jackson, C.L., 2002. Characteristics of intact and ruptured atherosclerotic plaques in brachiocephalic arteries of apolipoprotein $\mathrm{E}$ knockout mice. Arterioscler Thromb Vasc Biol, 22, 788-92. 
Xia, T., Kovochich, M., Liong, M., Madler, L., Gilbert, B., Shi, H., Yeh, J.I., Zink, J.I. \& Nel, A.E., 2008. Comparison of the mechanism of toxicity of zinc oxide and cerium oxide nanoparticles based on dissolution and oxidative stress properties. ACS Nano, 2, 2121-34.

Xue, Y., Zhai, Y., Zhou, K., Wang, L., Tan, H., Luan, Q. \& Yao, X., 2012. The vital role of buffer anions in the antioxidant activity of $\mathrm{CeO} 2$ nanoparticles. Chemistry, 18, 11115-22.

Yokel, R.A., Au, T.C., Macphail, R., Hardas, S.S., Butterfield, D.A., Sultana, R., Goodman, M., Tseng, M.T., Dan, M., Haghnazar, H., Unrine, J.M., Graham, U.M., Wu, P. \& Grulke, E.A., 2012. Distribution, elimination, and biopersistence to 90 days of a systemically introduced $30 \mathrm{~nm}$ ceria-engineered nanomaterial in rats. Toxicol Sci, 127, 256-68.

Yokel, R.A., Tseng, M.T., Dan, M., Unrine, J.M., Graham, U.M., Wu, P. \& Grulke, E.A., 2013. Biodistribution and biopersistence of ceria engineered nanomaterials: size dependence. Nanomedicine, 9, 398407.

Zhang, H., Ji, Z., Xia, T., Meng, H., Low-Kam, C., Liu, R., Pokhrel, S., Lin, S., Wang, X., Liao, Y.P., Wang, M., Li, L., Rallo, R., Damoiseaux, R., Telesca, D., Madler, L., Cohen, Y., Zink, J.I. \& Nel, A.E., 2012. Use of metal oxide nanoparticle band gap to develop a predictive paradigm for oxidative stress and acute pulmonary inflammation. ACS Nano, 6, 4349-68. 


\section{Supplementary information}

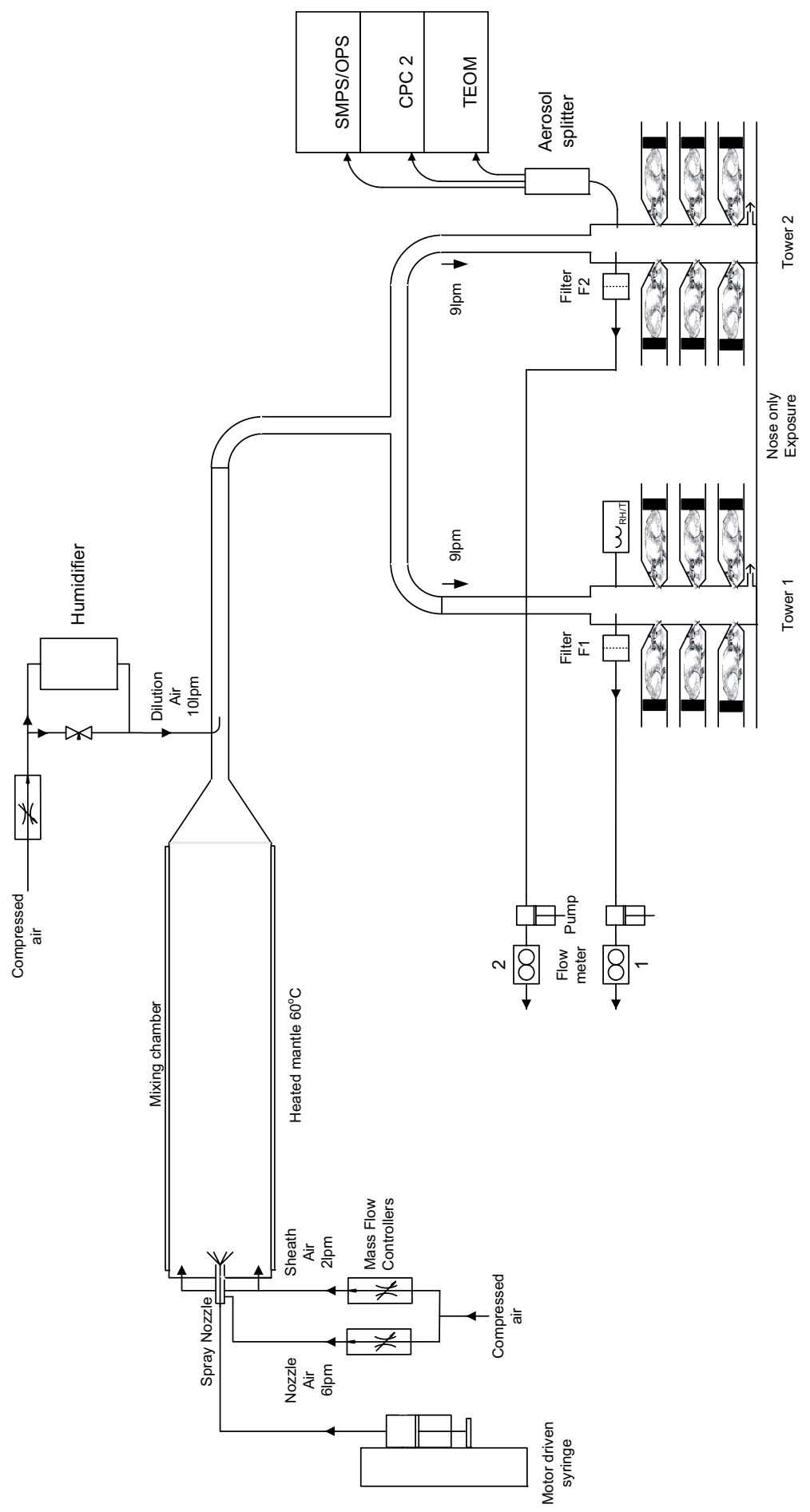

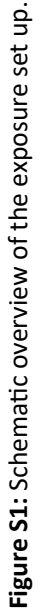




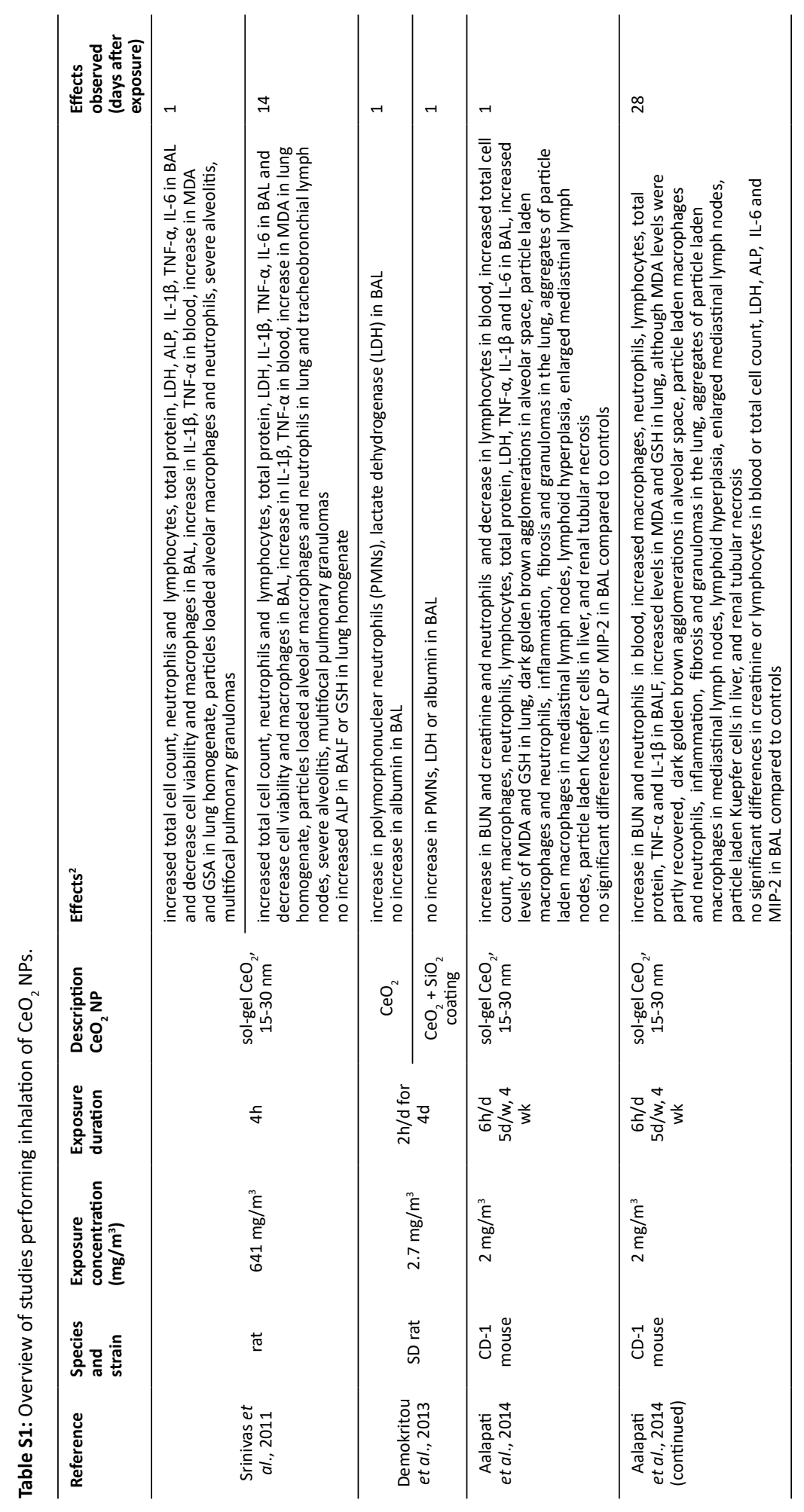




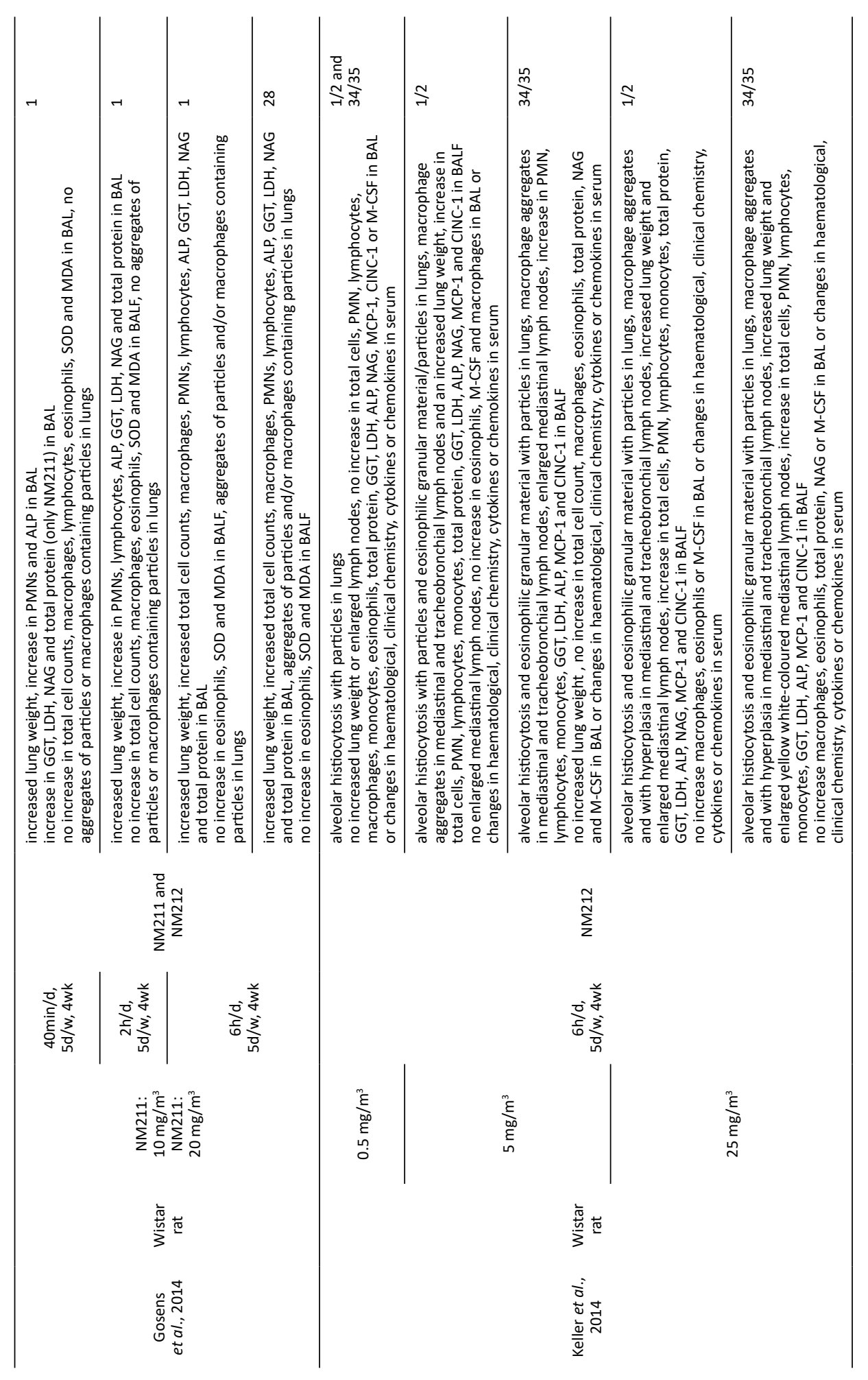




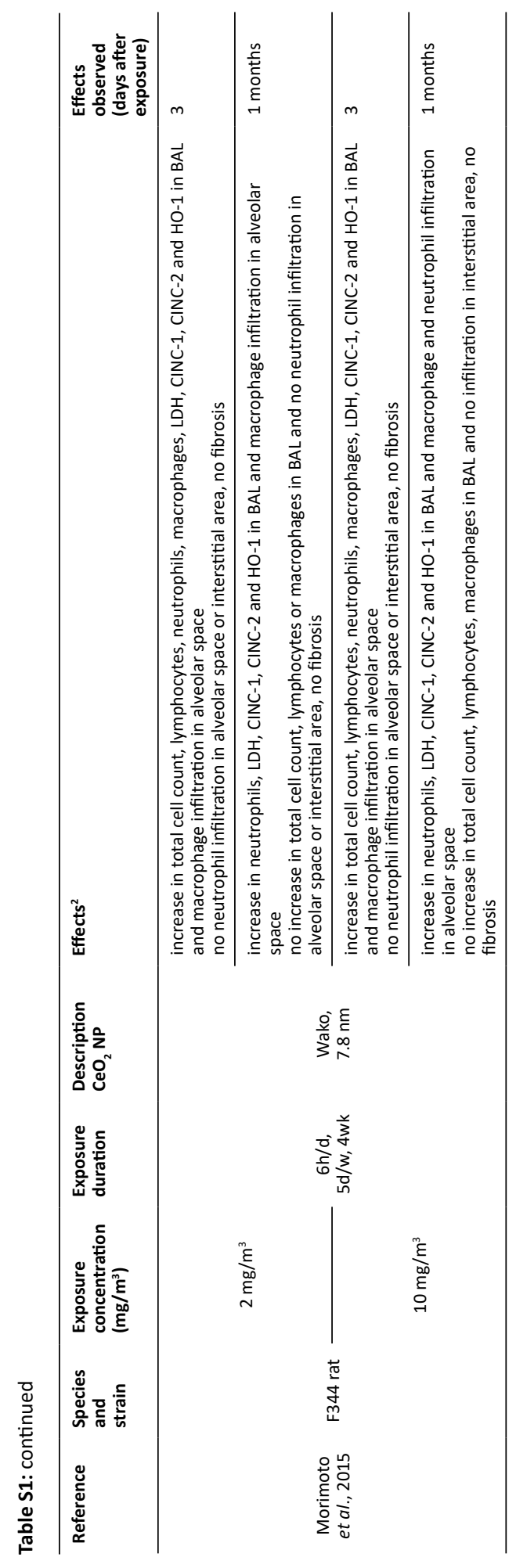




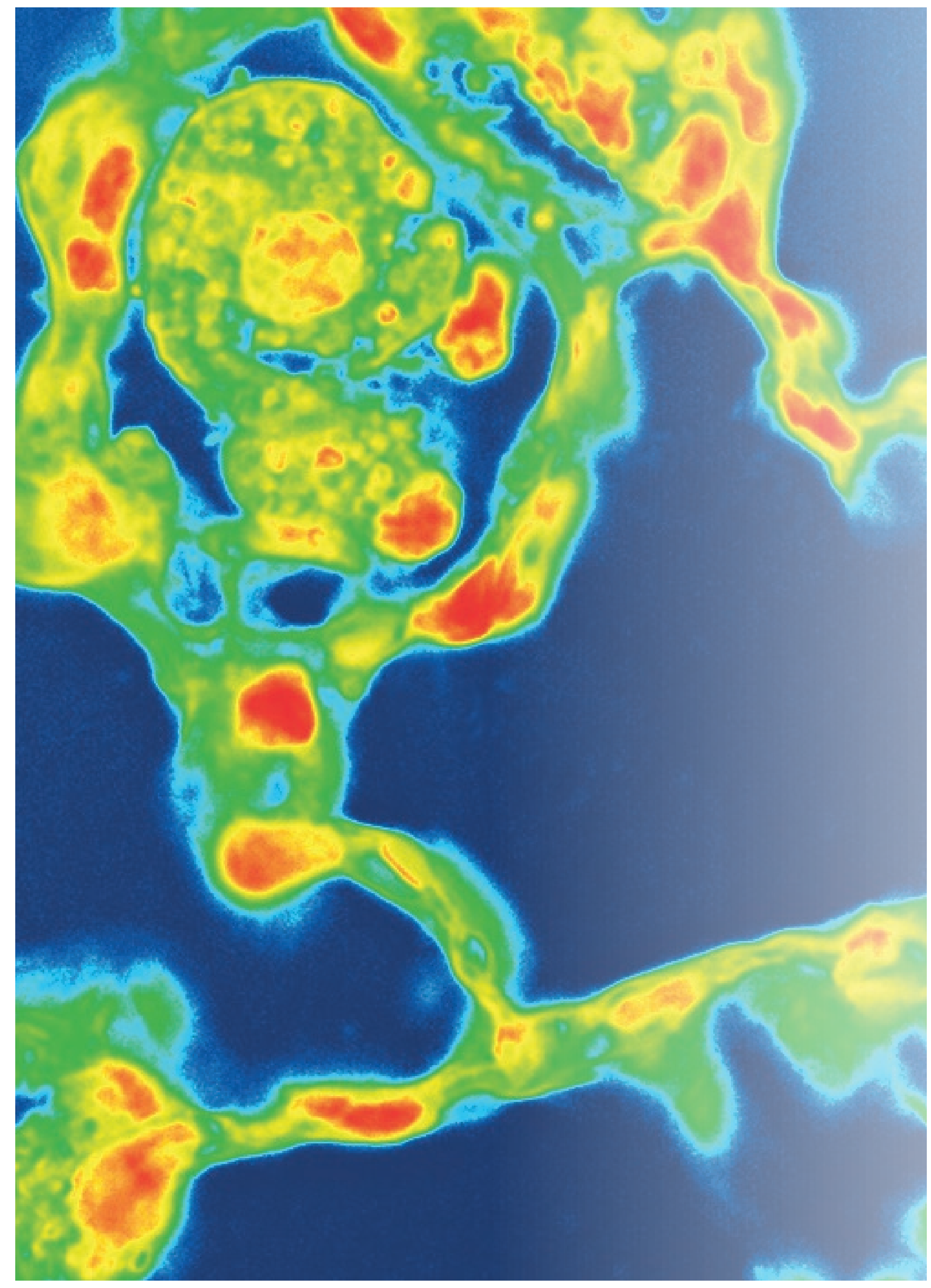




\section{Chapter 3}

\section{Differences in the toxicity of}

cerium dioxide nanomaterials after inhalation can be explained by lung deposition, animal species and nanoforms

Susan Dekkers' ${ }^{1}$ Lan Ma-Hock ${ }^{2}$, Iseult Lynch ${ }^{3}$, Mike Russ ${ }^{4}$, Mark R. Miller ${ }^{5}$, Roel P.F. Schins ${ }^{6}$, Jana Keller ${ }^{2}$, Isabella Römer ${ }^{3}$, Karin Küttler ${ }^{2}$, Volker Strauss ${ }^{2}$, Wim H. De Jong', Robert Landsiedel' ${ }^{2}$, Flemming R. Cassee ${ }^{1,7}$.

Inhalation Toxicology 30(7-8):273-286 (2018)

${ }^{1}$ National Institute for Public Health and the Environment, Bilthoven, The Netherlands,

2 Experimental Toxicology and Ecology, BASF SE, Ludwigshafen, Germany,

${ }^{3}$ School of Geography, Earth and Environmental Sciences, University of Birmingham, Edgbaston, UK,

${ }^{4}$ Promethean Particles Ltd., Nottingham, UK,

${ }_{5}^{5}$ Centre for Cardiovascular Science, University of Edinburgh, Edinburgh, UK,

6 IUF - Leibniz Research Institute for Environmental Medicine, Düsseldorf, Germany,

${ }^{7}$ Institute for Risk Assessment Sciences, Utrecht University, Utrecht, The Netherlands 


\begin{abstract}
Considerable differences in pulmonary responses have been observed in animals exposed to cerium dioxide nanoparticles via inhalation. These differences in pulmonary toxicity might be explained by differences in lung deposition, species susceptibility or physicochemical characteristics of the tested cerium dioxide nanoforms (i.e. same chemical substance, different size, shape, surface area or surface chemistry). In order to distinguish the relative importance of these different influencing factors, we performed a detailed analysis of the data from several inhalation studies with different exposure durations, species and nanoforms, namely published data on NM211 and NM212 (JRC repository), NanoAmor (commercially available) and our published and unpublished data on PROM (industry provided). Data were analyzed by comparing the observed pulmonary responses at similar external and internal dose levels. Our analyses confirm that rats are more sensitive to developing pulmonary inflammation compared to mice. The observed differences in responses do not result purely from differences in the delivered and retained doses (expressed in particle mass as well as surface area). In addition, the different nanoforms assessed showed differences in toxic potency likely due to differences in their physicochemical parameters. Primary particle and aggregate/agglomerate size distributions have a substantial impact on the deposited dose and consequently on the pulmonary response. However, in our evaluation size could not fully explain the difference observed in the analyzed studies indicating that the pulmonary response also depends on other physicochemical characteristics of the particles. It remains to be determined to what extent these findings can be generalized to other poorly soluble nanomaterials.
\end{abstract}




\section{Introduction}

The number and variety of nanomaterials is expected to continue to increase because of the adaptability of their physicochemical properties to enhance their functionality for many different applications. However, manipulating the physicochemical properties of nanomaterials may also lead to undesirable behavior and, possibly, harmful effects in those exposed. A challenge for health risk assessment is the rapidly growing variety of nanomaterials and nanoforms (nanomaterials of the same chemical substance, but with different physicochemical characteristics such as size, shape, surface area and surface chemistry (EC 2017; ECHA 2017)). Thus, in recent years there is an increasing interest in strategies to compare assessments across different nanoforms, including (Quantitative) Structure Activity Relationships, grouping and read-across (Zhang et al. 2012; Stone et al. 2014; Arts et al. 2015; Oomen et al. 2015). A fuller understanding of the influence of basic (physicochemical) properties of nanomaterials on their potential toxicity is needed for further development, validation and specifically implementation of new or revised approaches in health risk assessment (Dekkers et al. 2016).

Recently, we have investigated the influence of zirconium doping of cerium dioxide nanoparticles $\left(\mathrm{CeO}_{2} \mathrm{NPs}\right)$ on their biodistribution, pulmonary and cardiovascular effects in mice following inhalation (Dekkers et al. 2017). Sub-acute (4 weeks) inhalation of the undoped $\mathrm{CeO}_{2} \mathrm{NPs}\left(4 \mathrm{mg} / \mathrm{m}^{3}, 3 \mathrm{~h} / \mathrm{d}, 5 \mathrm{~d} / \mathrm{w}\right)$ resulted in minimal pulmonary and cardiovascular effects in mice 4 weeks post-exposure. Other toxicity studies showed more pronounced effects after exposure to $\mathrm{CeO}_{2}$ NPs under similar exposure conditions in rats (Geraets et al. 2012; Gosens et al. 2014; Keller et al. 2014; Arts et al. 2016) and mice (Aalapati et al. 2014). Possible explanations for the observed differences in toxicity of $\mathrm{CeO}_{2} \mathrm{NPs}$ after inhalation are: a) differences in kinetics (deposition and clearance) of the $\mathrm{CeO}_{2} \mathrm{NPs}$, b) differences in the susceptibility of mice compared to rats or c) differences in the toxicity of the different nanoforms of $\mathrm{CeO}_{2}$, due to differences in certain physicochemical characteristics such as size, shape and surface chemistry. The objective of the analysis described in this paper is to investigate which of these possible explanations could explain the relatively mild toxicity of the undoped $\mathrm{CeO}_{2}$ NPs observed in Dekkers et al. (2017) compared to the more pronounced toxicity observed in other inhalation studies.

The importance of toxicokinetics in interpreting pulmonary responses to inhaled NPs has been highlighted by several researchers (Kreyling et al. 2013; Kuempel et al. 2015; Pauluhn 2017). Previous studies, such as Braakhuis et al. (2016), indicate that the internal dose is more predictive of the toxicity of inhaled NPs than the external dose, suggesting that the deposition pattern (i.e. the regions of the respiratory tract in which NPs deposit) determines its interaction with the lung (e.g. lung lining fluid, cellular membranes and fluids), and the subsequent lung clearance and toxicity. 
Differences in the susceptibility of different species have been observed in several studies, showing rats to be more susceptible than mice to inhaled $\mathrm{TiO}_{2} \mathrm{NPs}$ and carbon black (Bermudez et al. 2004; Elder et al. 2005; Warheit et al. 2016). This difference has been attributed to differences in the deposition as well as the pulmonary response, especially at concentrations where macrophage-mediated clearance is impaired (pulmonary overload conditions) (Bermudez et al. 2004; Warheit et al. 2016). Since the occurrence of lung tumors under pulmonary overload conditions in rats may not be representative for humans, the use of rats for particle toxicology has been criticized (Warheit et al. 2016). Although pulmonary overload generally occurs at higher exposure concentrations in mice and other experimental animals, there is no consensus as to the most appropriate species to predict particle induced lung toxicity in humans. One major advantage of using mice is the availability of many disease models and relative ease of genetic modification to provide mechanistic insight.

Toxicokinetics and toxicodynamics of inhaled NPs are greatly influenced by their physical characteristics, such as the size and density of the primary particles, aggregates or agglomerates, as well as by their chemical composition, dissolution rate, surface reactivity, shape, charge and hydrophobicity (Nel et al. 2006; Braakhuis et al. 2014; Bakand and Hayes 2016).

To investigate the possible explanation(s) for the discrepancies between the findings of different $\mathrm{CeO}_{2} \mathrm{NP}$ inhalation studies, we have compared the results of exposure to undoped $\mathrm{CeO}_{2}$ NPs (herein referred to as PROM) from our recent study (Dekkers et al., 2017) to the results of other 4 week inhalation studies with various nanoforms of $\mathrm{CeO}_{2}$ (referred to as NM212, NM211 and NanoAmor in the subsequent tables and figures) in rats and mice (Geraets et al. 2012; Aalapati et al. 2014; Gosens et al. 2014; Keller et al. 2014). To investigate species differences in the susceptibility to $\mathrm{CeO}_{2} \mathrm{NPs}$, two additional 5 day inhalation studies using the same undoped $\mathrm{CeO}_{2}$ NPs as used in Dekkers et al. (2017) (PROM) were performed; one in rats and one in mice to bridge the results from the various studies. These additional studies are not presently published in peer-reviewed journals, but were performed within the NanoMILE project (http://nanomile.eu-vri.eu/) under GLP according a short term inhalation study (STIS) protocol, and were included in Deliverable D7.2 (NanoMILE 2017) which is available upon request. The STIS has been specifically designed for the testing of toxicity and kinetics of nanomaterials and has previously been applied for hazard assessment of 13 metal oxide nanomaterials, including the $\mathrm{CeO}_{2}$ nanoforms NM211 and NM212 (Keller et al. 2014; Landsiedel et al. 2014).

To investigate the origin of the differences in toxicity of the different $\mathrm{CeO}_{2}$ nanoforms, the results of these 5-day inhalation studies with the $\mathrm{PROM} \mathrm{CeO} \mathrm{NPs}_{2}$ were compared to the results of previous 5 day inhalation studies with other nanoforms of $\mathrm{CeO}_{2}$ (NM212 and NM211) in rats (Keller et al. 2014). 
This paper describes the analysis of data from the aforementioned $\mathrm{CeO}_{2} \mathrm{NP}$ inhalation studies to investigate to what extent the observed differences in toxicity between various studies can be explained by differences in kinetics, species susceptibility and/or physicochemical characteristics of the nanoforms.

We hypothesized that a lower internal dose and higher clearance of the $\mathrm{CeO}_{2} \mathrm{NPs}$ might explain the relatively mild effects observed 4 weeks post-exposure in our recent study (Dekkers et al. 2017). We also hypothesized that, similar to other poorly soluble particles (such as $\mathrm{TiO}_{2} \mathrm{NPs}$ and carbon black), rats would be more susceptible to exposure to $\mathrm{CeO}_{2}$ NPs than mice. Lastly, based on the mild toxicity of $\mathrm{PROM} \mathrm{CeO}_{2}$ NPs observed in mice, we hypothesized that PROM CeO $\mathrm{CPs}_{2}$ would also be less toxic compared to other nanoforms of $\mathrm{CeO}_{2}$ NPs in rats.

\section{Materials and methods}

\section{Selection of the Studies}

The selected studies were limited to those where rats or mice were exposed via inhalation (either whole body or nose only) and in which the pulmonary toxicity as well as pulmonary dose (i.e. lung burden) were determined. Studies in which rats and mice were exposed for 4 weeks as well as for 5 days were selected because not all nanoforms of $\mathrm{CeO}_{2}$ were tested in 4 week inhalation studies in both species. An overview of the inhalation studies that were analyzed can be found in Table 1. A summary of the pulmonary findings of these inhalation studies can be found in Table S1 of the supplementary information, and further details on the 5-day inhalation studies with the PROM CeO ${ }_{2} \mathrm{NPs}$ can be found in Table 4 and Annex S2.

\section{From External Concentration to Internal Lung or Pulmonary Burden}

To investigate the role of kinetics in pulmonary toxicity of $\mathrm{CeO}_{2} \mathrm{NPs}$, toxicity data of the studies was compared using a) the external exposure concentration, b) the internal retained dose in the lung (i.e. the measured lung burden) and $c$ ) the predicted internal retained dose in the pulmonary or alveolar regions of the lung (i.e. the expected pulmonary burden). In all studies, the rats or mice were exposed to $\mathrm{CeO}_{2} \mathrm{NPs}$ at concentrations between 0.5 and 25 $\mathrm{mg} / \mathrm{m}^{3}$ with similar particle size distributions (mass median aerodynamic diameter (MMAD) between 0.9 to $2.2 \mu \mathrm{m}$ ).

The measured lung burden (LB) was calculated from the measured Ce concentrations in the lung tissue (typically determined by ICP-MS) and total lung weight. For those studies in which no lung and body weights were reported (Aalapati et al. 2014; Keller et al. 2014), an estimation of the lung weight was made based on the lung weights of animals of the same strain and similar ages. 
Table 1: Overview of the selected $\mathrm{CeO}_{2} \mathrm{NP}$ inhalation toxicity studies.

\begin{tabular}{|c|c|c|c|c|c|c|c|c|}
\hline Reference & $\begin{array}{l}\text { Strain, species and } \\
\text { sex of animals }\end{array}$ & $\begin{array}{l}\xi \xi \\
\frac{1}{0} \\
\frac{0}{0} \\
\frac{\pi}{\pi}\end{array}$ & 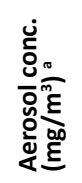 & 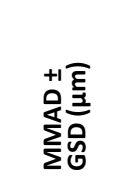 & 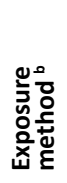 & 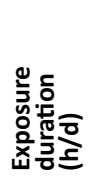 & 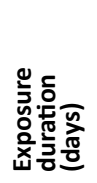 & 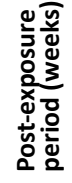 \\
\hline \multirow{2}{*}{$\begin{array}{l}\text { Keller } \\
\text { et al., } 2014\end{array}$} & \multirow{2}{*}{ Wistar rats, female } & \multirow{2}{*}{ NM211 } & 0.45 & $1.9 \pm 2.9$ & \multirow{2}{*}{ WB } & \multirow{2}{*}{6} & \multirow{2}{*}{5} & \multirow{2}{*}{3} \\
\hline & & & 25.8 & $2.2 \pm 2.4$ & & & & \\
\hline \multirow{3}{*}{$\begin{array}{l}\text { Keller } \\
\text { et al., } 2014\end{array}$} & \multirow{3}{*}{ Wistar rats, female } & \multirow{3}{*}{ NM212 } & 0.5 & $1.4 \pm 2.3$ & \multirow{3}{*}{ WB } & \multirow{3}{*}{6} & \multirow{3}{*}{5} & \multirow{3}{*}{3} \\
\hline & & & 5.3 & $1.2 \pm 2.1$ & & & & \\
\hline & & & 25.9 & $1.0 \pm 2.5$ & & & & \\
\hline \multirow{3}{*}{$\begin{array}{l}\text { NanoMILE, } \\
2017\end{array}$} & \multirow{3}{*}{ Wistar rats, male } & \multirow{3}{*}{ PROM } & 0.57 & $1.3 \pm 2.2$ & \multirow{3}{*}{ NO } & \multirow{3}{*}{6} & \multirow{3}{*}{5} & \multirow{3}{*}{3} \\
\hline & & & 2.04 & $1.2 \pm 2.4$ & & & & \\
\hline & & & 4.85 & $1.1 \pm 1.3$ & & & & \\
\hline \multirow{3}{*}{$\begin{array}{l}\text { Keller } \\
\text { et al., } 2014\end{array}$} & \multirow{3}{*}{ Wistar rats, male } & \multirow{3}{*}{ NM212 } & 0.48 & $1.6 \pm 2.1$ & \multirow{3}{*}{ WB } & \multirow{3}{*}{6} & \multirow{3}{*}{20} & \multirow{3}{*}{5} \\
\hline & & & 5.2 & $1.3 \pm 2.1$ & & & & \\
\hline & & & 25.6 & $0.9 \pm 2.5$ & & & & \\
\hline $\begin{array}{l}\text { Geraerts } \\
\text { et al., } 2012\end{array}$ & $\begin{array}{l}\text { Wistar rats, male + } \\
\text { female }\end{array}$ & NM211 & 10.8 & $1.0 \pm 1.8$ & NO & 6 & 20 & 4 \\
\hline $\begin{array}{l}\text { Geraerts } \\
\text { et al., } 2012\end{array}$ & $\begin{array}{l}\text { Wistar rats, male }+ \\
\text { female }\end{array}$ & NM212 & 19.95 & $1.2 \pm 2.1$ & NO & 6 & 20 & 4 \\
\hline \multirow{3}{*}{$\begin{array}{l}\text { NanoMILE, } \\
2017\end{array}$} & \multirow{3}{*}{$\begin{array}{l}\text { C57BL/6 J mice, } \\
\text { male }\end{array}$} & \multirow{3}{*}{ PROM } & 0.54 & $1.1 \pm 2.5$ & \multirow{3}{*}{ NO } & \multirow{3}{*}{6} & \multirow{3}{*}{5} & \multirow{3}{*}{3} \\
\hline & & & 2.04 & $1.1 \pm 2.4$ & & & & \\
\hline & & & 5.04 & $0.9 \pm 3.0$ & & & & \\
\hline $\begin{array}{l}\text { Dekkers } \\
\text { et al., } 2017\end{array}$ & $\begin{array}{l}\text { C57BL/6 J mice, } \\
\text { female }\end{array}$ & PROM & 3.98 & $0.3 \pm 1.6 c$ & NO & 3 & 20 & 4 \\
\hline $\begin{array}{l}\text { Aalipati } \\
\text { et al., } 2014\end{array}$ & CD-1 mice, male & $\begin{array}{l}\text { NanoA- } \\
\text { mor }\end{array}$ & 2.0 & $1.4 \pm 2.4$ & NO & 6 & 20 & $\begin{array}{c}2 \text { and } \\
4\end{array}$ \\
\hline
\end{tabular}

a These aerosol concentrations are rounded to $0.5,2,5,10,20$ and $25 \mathrm{mg} / \mathrm{m}^{3}$ in the main text.

${ }^{\mathrm{b}} \mathrm{WB}=$ whole body. $\mathrm{NO}=$ nose only.

'Mass median diameter (MMD) instead of MMAD, estimated based on the particle size distributions of the scanning mobility particle sizer (SMPS) measurements, assuming spherical aggregation around primary particles of $4.7 \mathrm{~nm}$. MMAD=mass median aerodynamic diameter. GSD=geometric standard deviation.

To verify if the different methods used to measure the LB resulted in comparable results, the anticipated LB was also estimated using the Multiple-Path Particle Dosimetry Model (MPPD V3.04, Applied Research Associates, Inc., https://www.ara.com/products/multiple-pathparticle-dosimetry-model-mppd-v-304). The chosen MPPD airway morphometry model and input data used to estimate the retained dose in the lung of the studies can be found in Table S3 of the supplementary information. For all studies the "deposition and clearance" option of the MPPD model was used to estimate the tracheobronchial retention (TBR) and the alveolar retention (AR). The density of the bulk material of $\mathrm{CeO}_{2}\left(7.215 \mathrm{~g} / \mathrm{m}^{3}\right)$ was used 
as input parameter as no information is available of the density of $\mathrm{CeO}_{2}$ nanoforms occurring in the aerosols. Default settings where used unless stated otherwise.

The estimated LB was calculated using the following formula:

Estimated $L B(\mu g / g)=\frac{T B R(\mu g)+A R(\mu g)}{\text { Lung weight }(g)}$

The expected pulmonary burden (PB) was expressed as the surface area of the NPs per surface area of the pulmonary region of the lung $\left(\mathrm{mm}^{2} \mathrm{NP} / \mathrm{cm}^{2}\right.$ lung) instead of $\mu \mathrm{g} \mathrm{NP} / \mathrm{g}$ lung, based on the findings of Braakhuis et al. (2016). PB was calculated using the measured LB, the fraction of deposition in the alveolar region of the lung $(A D)$, the total deposition in the lung (alveolar deposition + tracheobronchial deposition; AD+TBD) (as estimated with the MPPD model), the surface area of the NPs (SA ${ }_{\mathrm{NP}}$; estimated using the reported BET measurement or calculated based on primary particle size) and the surface area of the pulmonary region of the lung ( $\mathrm{SA}_{\mathrm{PR}}$; estimated with the MPPD model). The data used to calculate the PB of the selected studies can be found in Table S4 of the supplementary information. The PB was calculated using the following formula:

$P B\left(\mathrm{~mm}^{2} / \mathrm{nm}^{2}\right)=\frac{L B(\#) \times \frac{A D(\%)}{(A D(\%)+T B D(\%))} \times S A_{N P}\left(\mathrm{~m}^{2}\right)}{S A_{P R}\left(\mathrm{~cm}^{2}\right)}$

To investigate if differences in LB or PB might explain the differences in toxicity, bronchoalveolar lavage fluid (BALF) and histopathological analyses of the studies were quantitatively compared using the measured exposure concentration ( $\mathrm{mg} \mathrm{NP} / \mathrm{m}^{3}$ air), LB

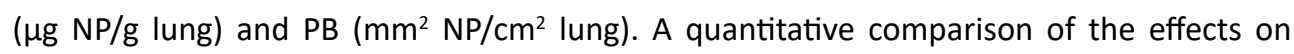
the BALF analyses among the studies was considered appropriate, since all studies used a similar method for lung lavage (2 lavages with a similar volume of saline solution). Alveolar histiocytosis (accumulation and infiltration of monocytes and macrophages in the pulmonary tissues) and increased neutrophil counts in BALF were also plotted against the exposure concentration, $\mathrm{LB}$ and $\mathrm{PB}$ to further examine dose response relationships, as these parameters were observed in most studies and are frequently identified as first evidence of adversity (Pauluhn 2017).

\section{Differences in Susceptibility}

To investigate species differences in susceptibility to the inflammatory effects induced by $\mathrm{CeO}_{2} \mathrm{NPs}$, and differences in toxicity between PROM CeO $\mathrm{NPs}_{2}$ and NM212, data from the two additional 5-day inhalation studies with the PROM CeO ${ }_{2}$ NPs in rats and mice (see Table 4 and Annex S2) were compared. 


\section{Statistical Analyses}

GraphPad Prism v7.00 (GraphPad Software, San Diego, California, USA) was used to visualize and to analyze the data using linear regression analysis of the measured versus the estimated LB and non-linear regression analysis (curve-fitting) of the dose response curves using the various dose metrics.

\section{Results and Discussion}

\section{Internal Lung and Pulmonary Burden}

An overview of the measured and estimated LB and expected PB from the selected $\mathrm{CeO}_{2}$ NP inhalation studies can be found in Table 2. Expressing the LB relative to the total lung weight and $\mathrm{PB}$ relative to the total surface area of the lung might lead to an underestimation of the LB and PB if exposure to the $\mathrm{CeO}_{2} \mathrm{NPs}$ also significantly increase the lung weight and/or the lung surface area. However, expressing the absolute LB and PB levels does not allow comparison of LB and PB levels of different studies, due to species, strain, age and bodyweight differences of the animals. Approximately 9 to $18 \%$ of the inhaled (mass) dose was predicted to deposit in the lungs, of which between 50 and $70 \%$ was expected to deposit in the pulmonary region of the lung (see Table $\mathrm{S} 4$ in the supplementary information). The measured LB was lower than the estimated LB at nearly all exposure concentrations. This overestimation of the LB by the MPPD models might be due to an underestimation of the clearance rates or an overestimation of the deposition. The clearance rates of the MPPD model are based on LB measurements from studies in which various strains of rats (F344) and mice (BALB/C and B6C3F1) were exposed to various micro- and nanosized particles $\left(\mathrm{TiO}_{2}, \mathrm{C} 60\right.$-fullerenes and $\left.\mathrm{Fe}_{x} \mathrm{O}_{y}\right)$ at various concentrations $\left(0.5,2,10\right.$ and $\left.20 \mathrm{mg} / \mathrm{m}^{3}\right)$, exposure durations ( $4 \mathrm{~h}$ and 13 weeks) and recovery periods ( 0 and $6 \mathrm{~h}, 1$ day, and 2, 4, 8, 13, 26 and 52 weeks). The animal strains (Wistar rats, C57BI/6 J and CD-1 mice), NPs $\left(\mathrm{CeO}_{2} \mathrm{NPs}\right)$, concentrations $\left(0.5,2,5,10,20\right.$ and $\left.25 \mathrm{mg} / \mathrm{m}^{3}\right)$, exposure duration ( 5 days and 4 weeks) and recovery periods ( 0 days and 3-5 weeks) of the selected studies only partly overlap with those on which the clearance rates of the MPPD model are based. Therefore, the high LBs estimated by the MPPD model indicate that the clearance rates might have been underestimated by the MPPD model, especially for the mice. Additionally, the deposition may have been overestimated. Next to differences in strains, concentrations and exposure durations between the selected studies and the studies on which the deposition in the MPPD model is based, also other assumptions may have led to an overestimation of deposition. For example, the model assumes spherical aerosols, while aerosols of NPs generally consist of non-spherical agglomerates of (spherical or nearly-spherical) primary particles, which tend to have a lower density than (solid) spherical particles of the same diameter. An overestimation of the density might lead to an overestimation of the amount deposited in the lungs. 


\section{Differences due to Deposition and Clearance}

To investigate to what extent the differences in toxicity may be explained by differences in pulmonary deposition and clearance of the $\mathrm{CeO}_{2} \mathrm{NPs}$, the LB and PB of several 4 week inhalation studies with various nanoforms of $\mathrm{CeO}_{2}$ (NM211, NM212, PROM and NanoAmor) in rats and mice are compared. A comparison of the measured and estimated LB in rats and mice after 4 weeks of exposure to $\mathrm{CeO}_{2}$ is presented in Figure 1.

Table 2: Measured and estimated lung burden and expected pulmonary burden of the selected inhalation studies.

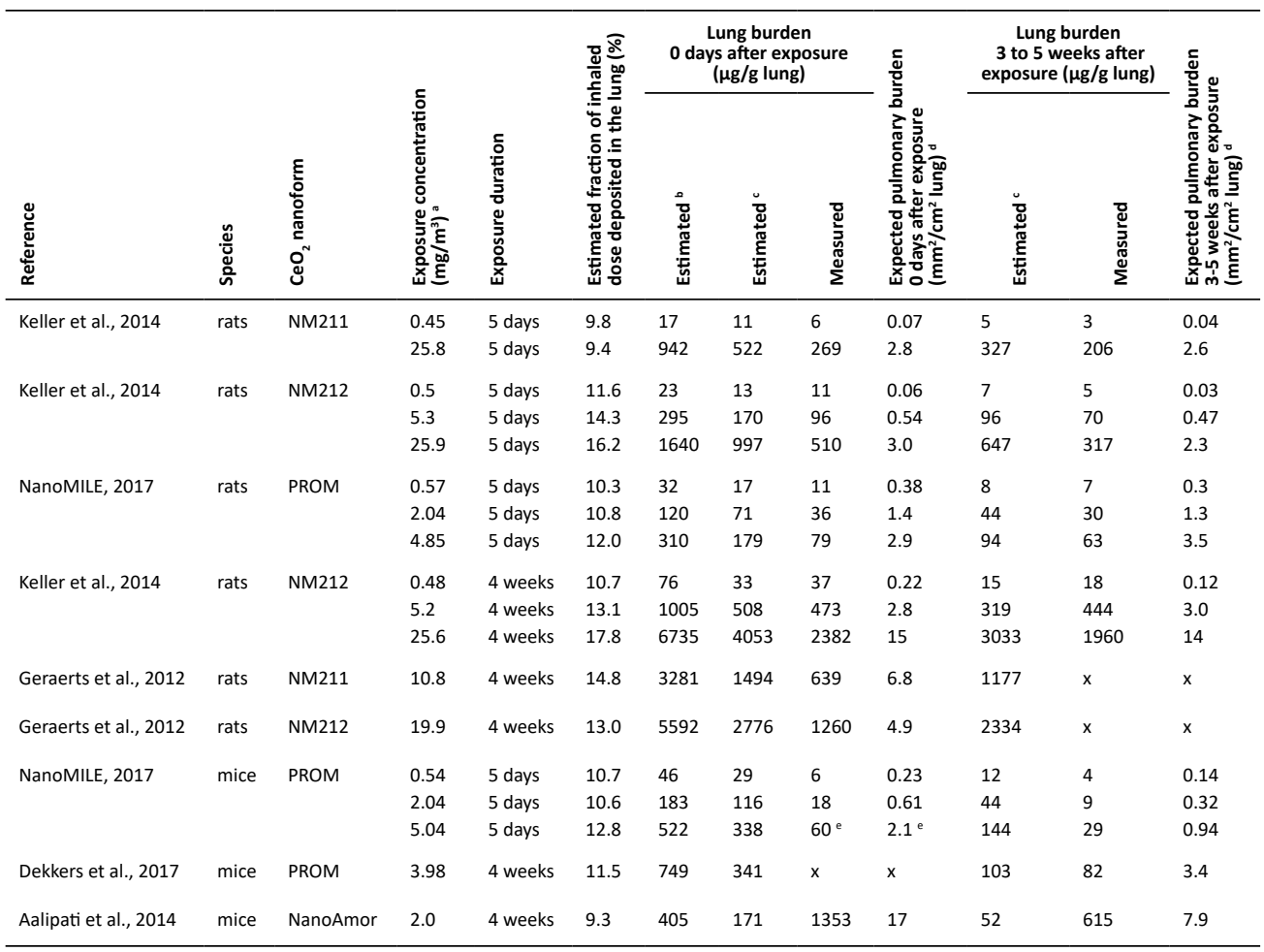

a These aerosol concentrations are rounded to $0.5,2,5,10,20$ and $25 \mathrm{mg} / \mathrm{m}^{3}$ in the main text.

${ }^{\mathrm{b}}$ Estimated with the MPPD model V3.04 (deposition only).

c Estimated with the MPPD model V3.04 (deposition and clearance).

${ }^{d}$ Calculated using the measured lung burden, estimated pulmonary deposition fractions and estimated surface area of the pulmonary region of the lung, as described in the materials and methods section.

e Estimated using the measured lung burden 21 days after exposure and the expected clearance $(\approx 50 \%)$. The measured lung burden 0 days after exposure (of $495 \mu \mathrm{g} / \mathrm{g}$ lung) was considered unreliable, and was probably a preparation artefact (e.g. big bronchus included in the sample). $x=$ no data available. 


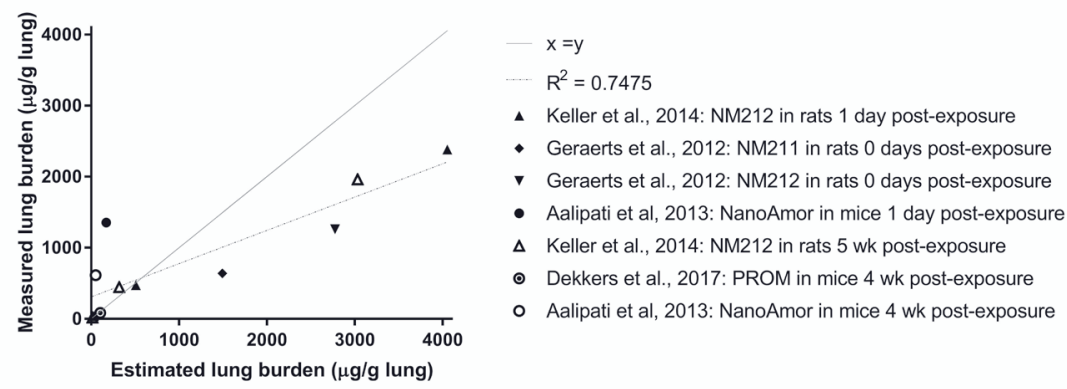

Figure 1: Measured lung burden plotted against the estimated lung burden in rats and mice after 4 weeks of exposure to various $\mathrm{CeO}_{2}$ NPs (NM211, NM212, PROM and NanoAmor). The estimated lung burden was assessed using the MPPD V3.04 model.

The measured LB correlated well $\left(R^{2}=0.75\right)$ with the estimated LB (Figure 1$)$, although this correlation was dominated by studies in rats. The measured LB was mostly lower or similar than the estimated LB, except for mice exposed to $2 \mathrm{mg} / \mathrm{mm}^{3}$ NanoAmor for which the measured LB was approximately 10 times higher than estimated (see black and white dot in Figure 1). There was no consistent relationship between the exposure concentration or particle diameter and the percentage of clearance $\left(R^{2}<0.5\right.$; data not shown).

Subsequently, it was investigated whether the differences in toxicity observed in the different 4 week inhalation studies with various nanoforms of $\mathrm{CeO}_{2}$ in rats and mice can be explained by differences in toxicokinetics (LB and PB). Therefore, a quantitative comparison was performed of the markers for toxicity (BALF and histopathological analyses) using external as well as internal concentrations expressed in two different dose metrics (Table 3 and Figure 2). All but one study found aggregates of particles and macrophages in the lungs, whereas only one study (in which rats were exposed to NM212) demonstrated an increase in the presence of eosinophilic material, interpreted to be indicative of cell damage. Granulomatous inflammation, indicative of macrophage mediated inflammation, was noted in only two studies (in which rats were exposed to NM212 and mice to NanoAmor). More importantly, neutrophilic inflammation was observed in all studies. The severity of the pulmonary toxicity (alveolar histiocytosis in $20 \%$ of the animals) observed in mice 4 weeks post-exposure to PROM CeO $\mathrm{NPs}_{2}$ (Dekkers et al. 2017) was remarkably less than the severity of the pulmonary toxicity (increase in neutrophils and lymphocytes in BALF, alveolar histiocytosis in $100 \%$ of the animals and the occurrence of eosinophilic granular material, macrophage aggregates with particles and granulomatous inflammation) observed in the other 4 week inhalation studies at similar or even lower external and internal concentrations (i.e. in rats exposed to NM211 and NM212 and mice exposed to NanoAmor). These findings indicate that differences in toxicity cannot be explained by differences in toxicokinetics alone.

When the toxicological effects were expressed as percentages of neutrophils in the BALF at each exposure concentration, non-linear curve fitting did not provide a suitable fit $\left(R^{2}=0.57\right.$ 
and 0.48; Figure 2a). Non-linear curve fitting was improved by plotting $\%$ neutrophils against internal dose, for the measured $L B\left(R^{2}=0.70\right.$ and 0.96 ; Figure $\left.2 c\right)$ but not for the $P B\left(R^{2}=0.55\right.$ and 0.56; Figure 2e). However, since the LB of the Aalapati study was approximately 10 times higher than the estimated LB (Figure 1 and Table 2), the LB and PB of this study could be considered as outliers. Without the data of the Aalapati study, the non-linear curve fitting led to better goodness of fit values for both the $L B\left(R^{2}=0.71\right.$ and 0.97$)$ and $P B\left(R^{2}=0.75\right.$ and $0.71)$ compared to the exposure concentration $\left(R^{2}=0.66\right.$ and 0.52$)$. Although these curve fitting analyses did not indicate whether LB ( $\mu \mathrm{g} / \mathrm{g}$ lung) or PB $\left(\mathrm{mm}^{2} / \mathrm{cm}^{2}\right)$ is a better predictor for the observed toxicity, they are in line with previous studies that indicated that the internal dose was more predictive of the toxicity of inhaled NPs than the external dose (Kreyling et al. 2013; Kuempel et al. 2015; Braakhuis et al. 2016; Pauluhn 2017). Nevertheless, the differences in toxicity may to some extent also be explained by differences in susceptibility between mice and rats exposed to similar internal dose levels and/or differences in toxicity between the various nanoforms of $\mathrm{CeO}_{2}$.
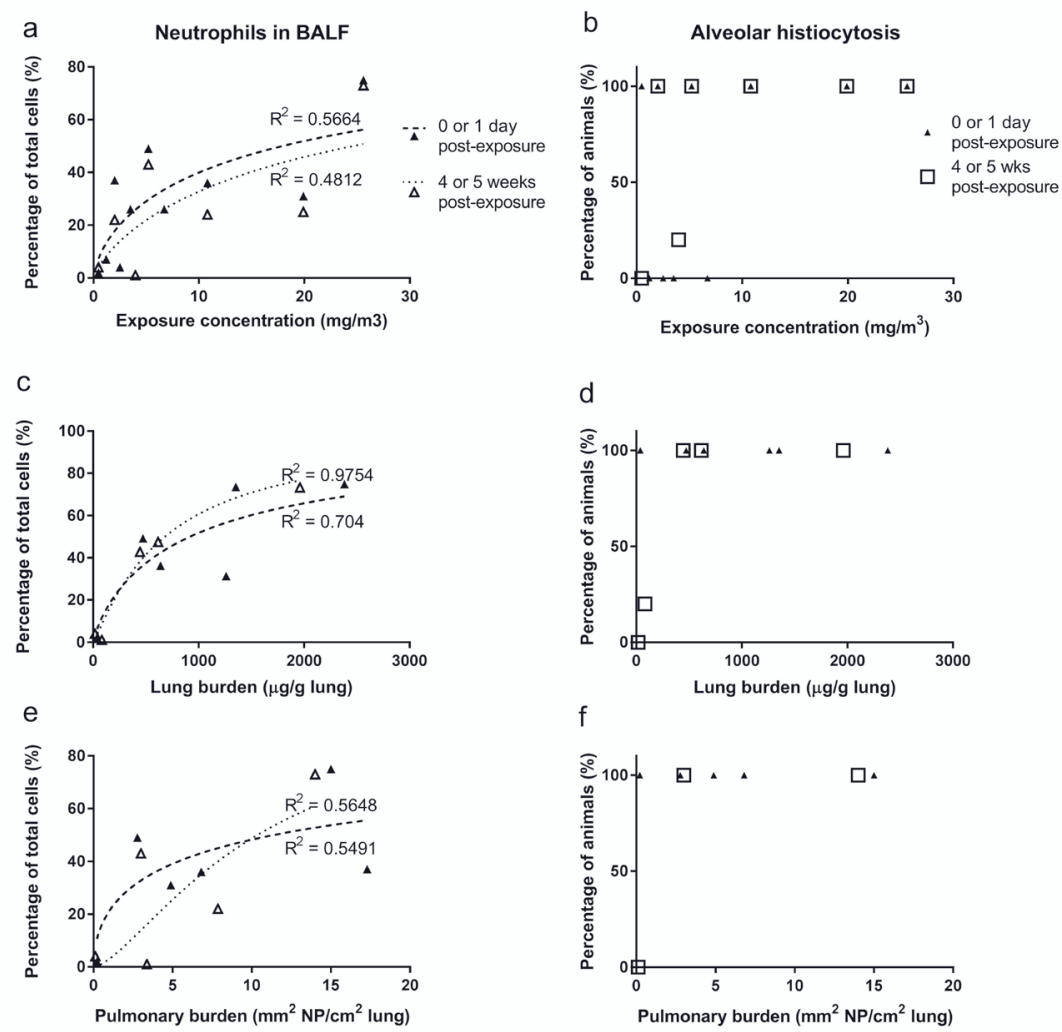

Figure 2: The percentage of neutrophils in the $\operatorname{BALF}(a, c$, and e) and the percentage of animals with alveolar histiocytosis ( $b, d$, and $f$ ) after 4 weeks of exposure to various $\mathrm{CeO}_{2} \mathrm{NPs}$ plotted against the measured exposure concentration ( $\mathrm{mg} \mathrm{NP} / \mathrm{m}^{3}$ air) ( $a$ and $b$ ), lung burden ( $\mu \mathrm{g} \mathrm{NP} / \mathrm{g}$ lung) (c and d) and expected pulmonary burden $\left(\mathrm{mm}^{2}\right.$ $\mathrm{NP} / \mathrm{cm}^{2}$ lung) (e and f). Data from all 4 week inhalation studies in rats and mice with various nanoforms of $\mathrm{CeO}_{2}$ (NM211, NM212, PROM and NanoAmor, as described in Table 3) were used. 


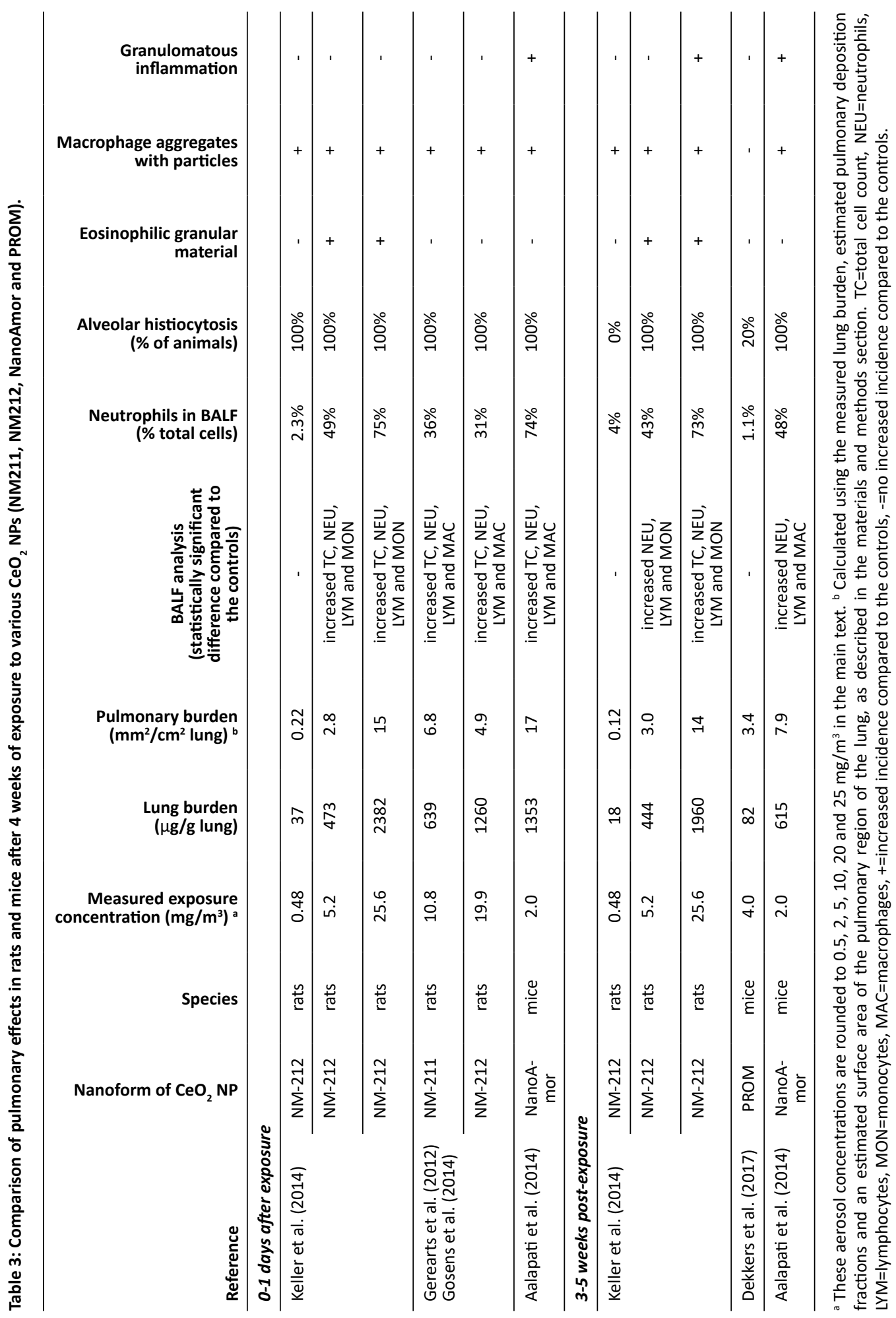




\section{Difference in Susceptibility between Mice and Rats}

To further investigate differences in the susceptibility of mice compared to rats, two additional 5 day inhalation studies with the same nanoform of $\mathrm{CeO}_{2}$ (PROM) were performed; one in mice and one in rats. These additional studies are not presently published in peerreviewed journals, but were performed within the NanoMILE project (http://nanomile.euvri.eu/) under GLP and were included in Deliverable D7.2 which is available upon request (NanoMILE 2017). The study design of these additional studies with $\mathrm{PROM} \mathrm{CeO}_{2} \mathrm{NPs}_{\text {follow }}$ the previously performed 5 day inhalation studies with NM212 (Keller et al. 2014), but used nose-only exposure instead of whole body exposure, to allow better comparison with the 4 week inhalation study with PROM $\mathrm{CeO}_{2}$ NPs in mice (Dekkers et al. 2017). Animals were exposed to similar concentrations of $\mathrm{CeO}_{2} \mathrm{NPs}\left(0.5,2\right.$ and $\left.5 \mathrm{mg} / \mathrm{m}^{3}\right)$ with similar particle size distributions (MMAD between 1.0 and $1.3 \mu \mathrm{m}$ for rats and between 0.9 and $1.2 \mu \mathrm{m}$ for mice). A detailed description of these studies can be found in Annex S2 of the supplementary information.

In male Wistar rats, exposure to $\mathrm{PROM} \mathrm{CeO}_{2}$ NPs led to changes of several BALF parameters, including increased neutrophils, in animals exposed to $2 \mathrm{mg} / \mathrm{m}^{3}$ and $5 \mathrm{mg} / \mathrm{m}^{3} \mathrm{CeO}_{2} \mathrm{NPs}$ (Table 4 ), the effects of which were still observed 3 weeks post-exposure. At these concentrations, the number of neutrophils was also increased in blood, as were blood monocyte counts. These findings indicate that inflammation in the lungs was persistent, and was accompanied by systemic inflammation. Histopathological findings after the recovery period were only observed at $5 \mathrm{mg} / \mathrm{m}^{3}$. The findings were consistent with a retarded lung clearance after 2 and $5 \mathrm{mg} / \mathrm{m}^{3} \mathrm{CeO}_{2} \mathrm{NP}$ exposure.

In mice, exposure to PROM $\mathrm{CeO}_{2} \mathrm{NPs}$ up to $5 \mathrm{mg} / \mathrm{m}^{3}$ did not lead to any significant change in clinical pathology parameters in blood and in BALF, or treatment-related adverse effects based on histopathology in mice. These findings were consistent with the higher clearance in mice (approximately 30 to $50 \%$ in 3 weeks) compared to rats (approximately 20 to $35 \%$ in 3 weeks).

The measured LB of the two 5 day inhalation studies was much lower than the estimated LB, but showed a high correlation with the estimated $L B\left(R^{2}=0.93\right.$ for rats and $R^{2}=0.91$ for mice; Figure 3). The high LBs estimated by the MPPD model indicate that some of the assumptions made by the MPPD model may not necessarily reflect the reality for the rats and mice in the selected studies and that the assumed clearance rate might have been underestimated by the MPPD model, especially in mice. 


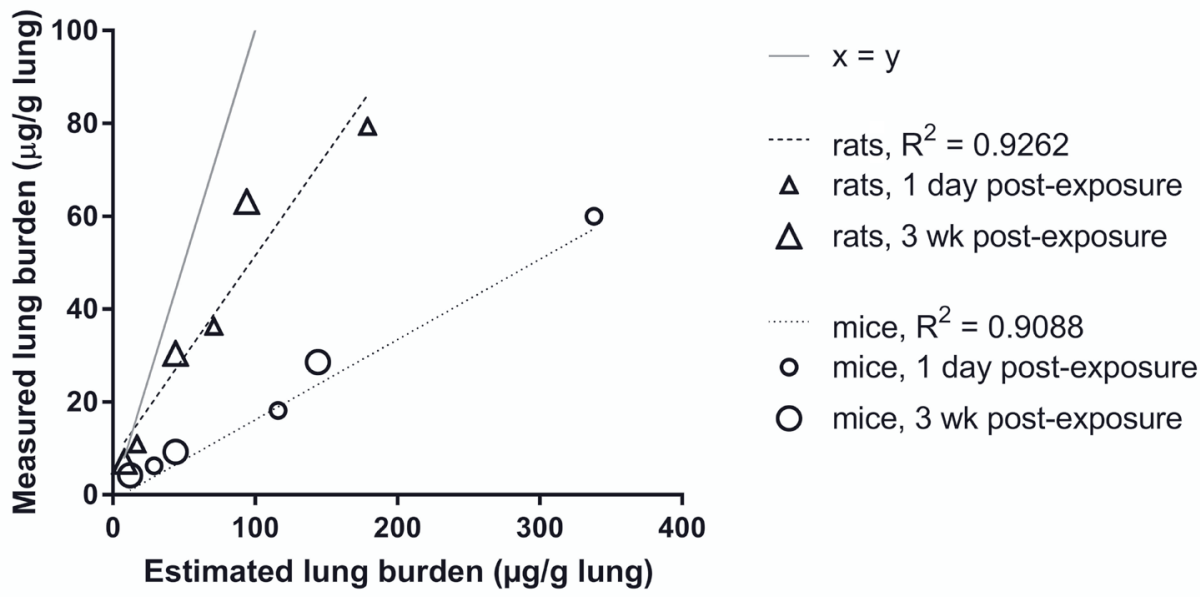

Figure 3: Measured lung burden plotted against the estimated lung burden in rats and mice after 5 days of exposure to PROM $\mathrm{CeO}_{2}$ NPs. The estimated lung burden was determined using the MPPD model.

The LB and PB was higher in rats compared to mice, especially 3 weeks post-exposure, an effect that is probably due to the higher clearance rates in mice compared to rats. To investigate if the modest toxicity of the PROM $\mathrm{CeO}_{2}$ NPs can be explained by the species difference in toxicokinetics, a quantitative comparison of pulmonary effect parameters was performed using external as well as internal concentrations (Table 4 and Figure 4). Similar to the curve fitting analyses of the 4 week inhalation studies (Figure $2 \mathrm{c}$ and e), the curve fitting analyses of the 5 day inhalation studies with PROM CeO $\mathrm{NPs}_{2}$ in rats and mice (Figure 4c and e) did not indicate whether LB ( $\mu \mathrm{g} / \mathrm{g}$ lung) or PB $\left(\mathrm{mm}^{2} / \mathrm{cm}^{2}\right)$ is a better predictor for the observed toxicity. The differences in internal concentration (LB and PB) cannot fully explain the differences in the severity of the toxicological effects, since changes in BALF and adverse histopathological effects were observed at lower LB and PB levels in rats compared to mice. Furthermore, the dose response curves are steeper in rats compared to mice, indicating PROM CeO $\mathrm{NPs}_{2}$ are more toxic to rats compared to mice (see Figure 4). This indicates that besides differences in kinetics (resulting in differences in LBs and PBs) between mice and rats, there are other differences in susceptibility between these species. This is in line with outcomes from previous studies in which rats tended to be more susceptible to pulmonary inflammation induced by poorly soluble particles (Bermudez et al. 2004; Borm et al. 2015; Warheit et al. 2016). There are no in vitro studies available that compare the toxicity of $\mathrm{CeO}_{2}$ NPs in mice and rat macrophages. However, the sensitivity of rat alveolar macrophages to various NPs has recently been tested in an in vitro assay to predict short-term inhalation of NPs. All four $\mathrm{CeO}_{2}$ NPs tested in this study (including NM211 and NM212) showed a dose dependent cytotoxicity and were identified as being biologically active in rat alveolar macrophages (Wiemann et al. 2016). 


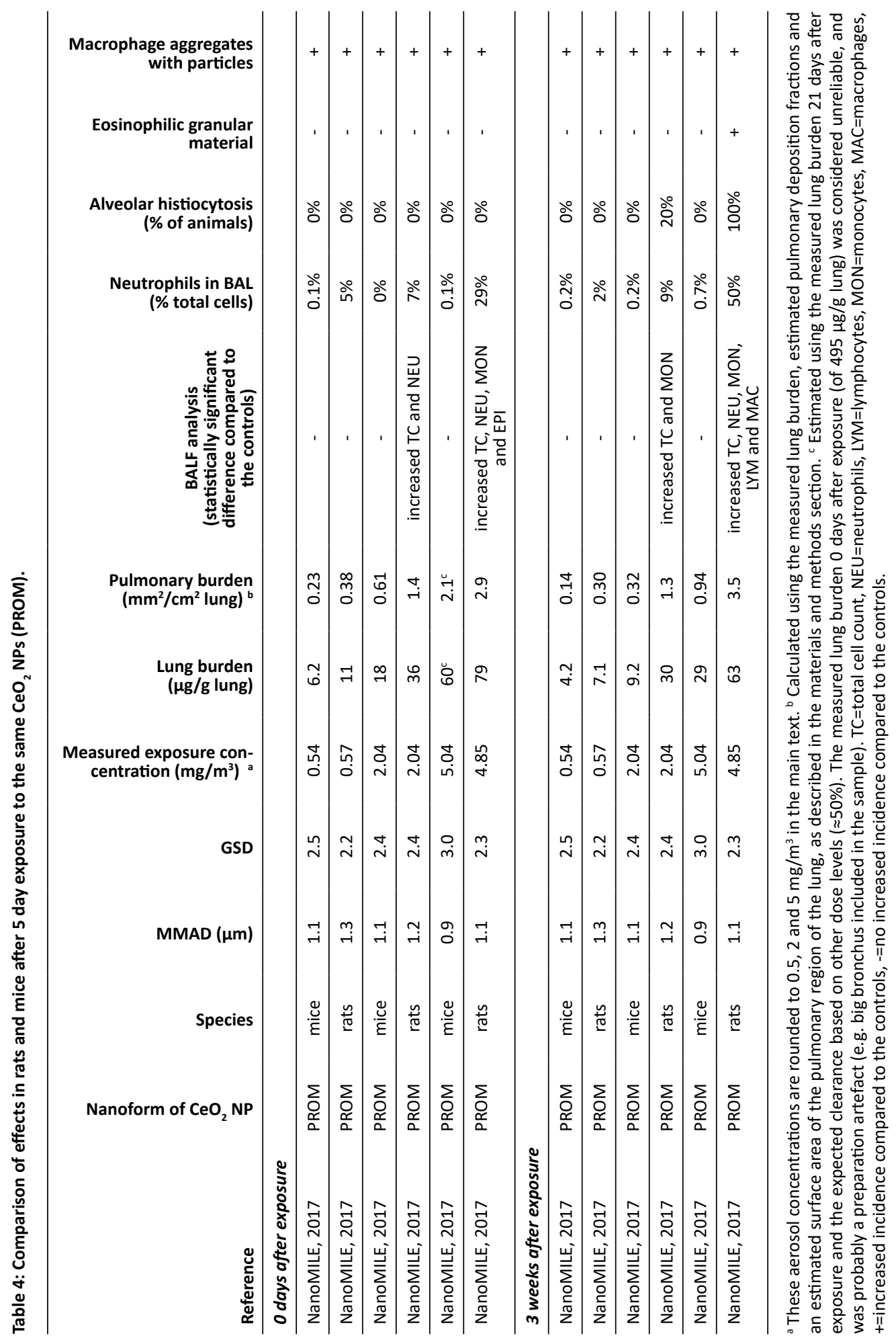




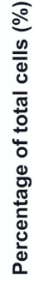

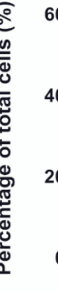

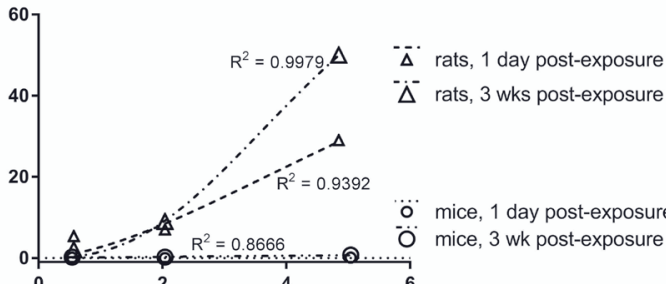

Measured exposure concentration $\left(\mathrm{mg} / \mathrm{m}^{3}\right)$

c
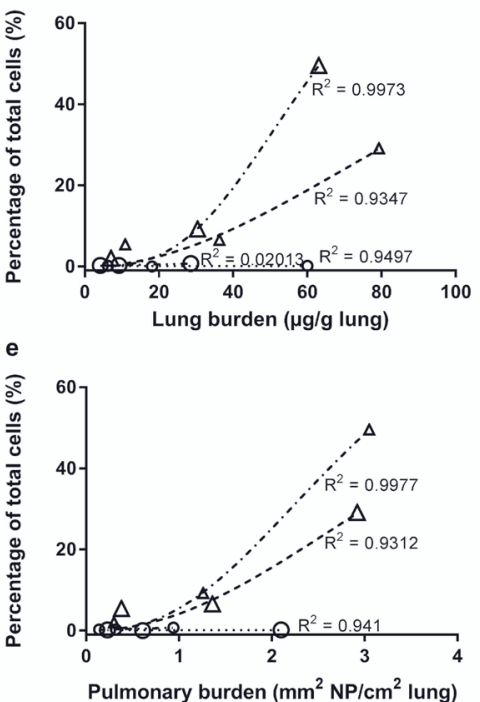

b

Alveolar histiocytosis

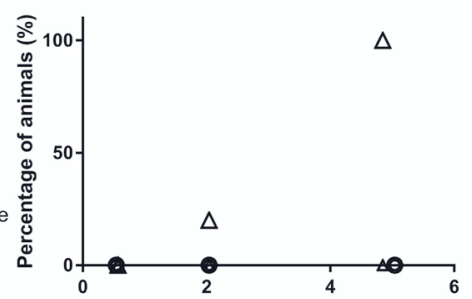

Measured exposure concentration $\left(\mathrm{mg} / \mathrm{m}^{3}\right)$

d

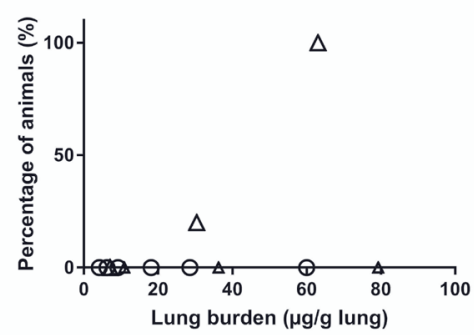

f

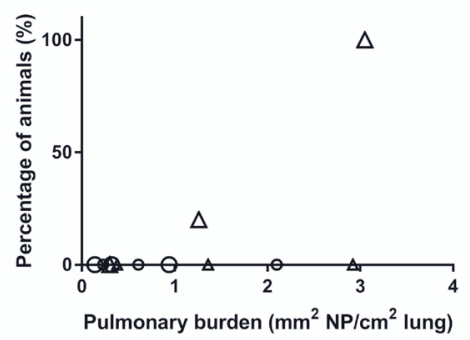

Figure 4: The percentage of neutrophils in the $\operatorname{BALF}(a, c$, and e) and the percentage of animals with alveolar

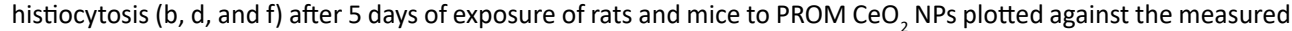
exposure concentration ( $\mathrm{mg} \mathrm{NP} / \mathrm{m}^{3}$ air) ( $\mathrm{a}$ and b), lung burden ( $\mu \mathrm{g} \mathrm{NP} / \mathrm{g}$ lung) ( $c$ and $\mathrm{d}$ ) and expected pulmonary burden ( $\mathrm{mm}^{2} \mathrm{NP} / \mathrm{cm}^{2}$ lung) (e and f).

\section{Differences due to Different Nanoforms}

To investigate differences in toxicity between the different $\mathrm{CeO}_{2}$ nanoforms, data from all 5 day inhalation studies in rats with NM211, NM212 and PROM were compared using exposure concentration, $\mathrm{LB}$ and $\mathrm{PB}$ and dose matrices. The measured LB was lower than the estimated LB but showed a strong correlation $\left(R^{2}=0.99\right.$, Figure 5$)$. 


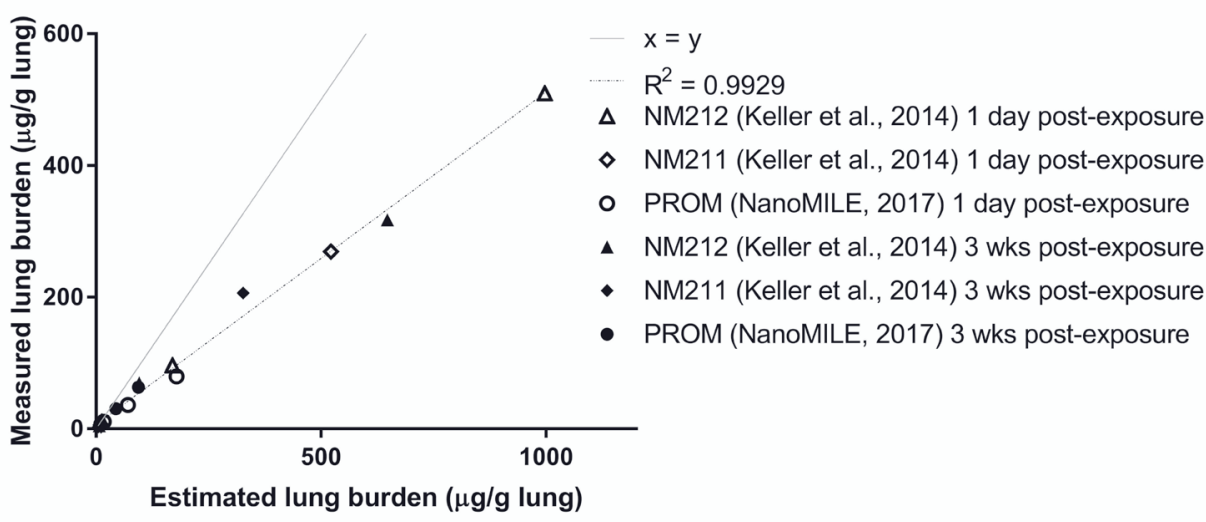

Figure 5: Measured lung burden plotted against the estimated lung burden in rats after 5 days of exposure to various $\mathrm{CeO}_{2} \mathrm{NPs}$ (NM211, NM212 and PROM). The estimated lung burden was determined using the MPPD model.

Exposure to PROM $\mathrm{CeO}_{2}$ NPs resulted in relatively high PB levels compared to NM211 and NM212 at similar exposure concentrations (Table 5 and Figure 6). This difference in PB in rats is probably caused by differences in clearance, since the measured LB levels and the estimated fractions of the inhaled dose deposited in the lung and pulmonary region of the lung were largely similar across the different $\mathrm{CeO}_{2}$ nanoforms (see Table S4). Clearance of particles deposited in the lungs is known to depend mainly on the dose, size, shape and dissolution rate of primary particles and their aggregates and agglomerates (Greim and Ziegler-Skylakakis 2007; Keller et al. 2014). Previous studies investigating the influence of particle size on the clearance have either found no influence of particle size on clearance (Semmler et al. 2004; Buckley et al. 2017) or a slower clearance of smaller particles (Geraets et al. 2012; Keller et al. 2014; Han et al. 2015). Indeed, the nanoforms with the smallest primary particle size (PROM: $4.7 \mathrm{~nm}$ ) seem to have a slower clearance than the larger nanoforms (NM211: $12.5 \mathrm{~nm}$ and NM212: $40 \mathrm{~nm}$; see Table S4 and S5 of supplementary information). However, the size of the aerosols has only a modest influence on the clearance rate, with the largest aerosols (NM211) showing a slightly higher clearance rate at all exposure concentrations compared to nanoforms with a smaller MMAD (NM212 and PROM) (see Table S4 and S5 of supplementary information).

To investigate if differences in toxicokinetics across the various nanoforms of $\mathrm{CeO}_{2}$ might explain the differences in toxicity, a quantitative comparison of parameters measured in BALF as well as histopathological analyses were performed using external as well as internal concentrations of NM211, NM212 and PROM (Table 5 and Figure 7). Similar to the curve fitting analyses of the 4 week inhalation studies (Figure $2 \mathrm{c}$ and e) and the 5 day inhalation studies with PROM CeO $\mathrm{NPs}_{2}$ in rats and mice (Figure $4 \mathrm{c}$ and e), curve fitting analyses of the 5 day inhalation studies in rats with the various $\mathrm{CeO}_{2}$ NPs (Figure $7 \mathrm{c}$ and e) did not indicate 
whether LB ( $\mu \mathrm{g} / \mathrm{g}$ lung) or PB $\left(\mathrm{mm}^{2} / \mathrm{cm}^{2}\right)$ is a better predictor for the observed toxicity. More data is needed to investigate the best dose metric.

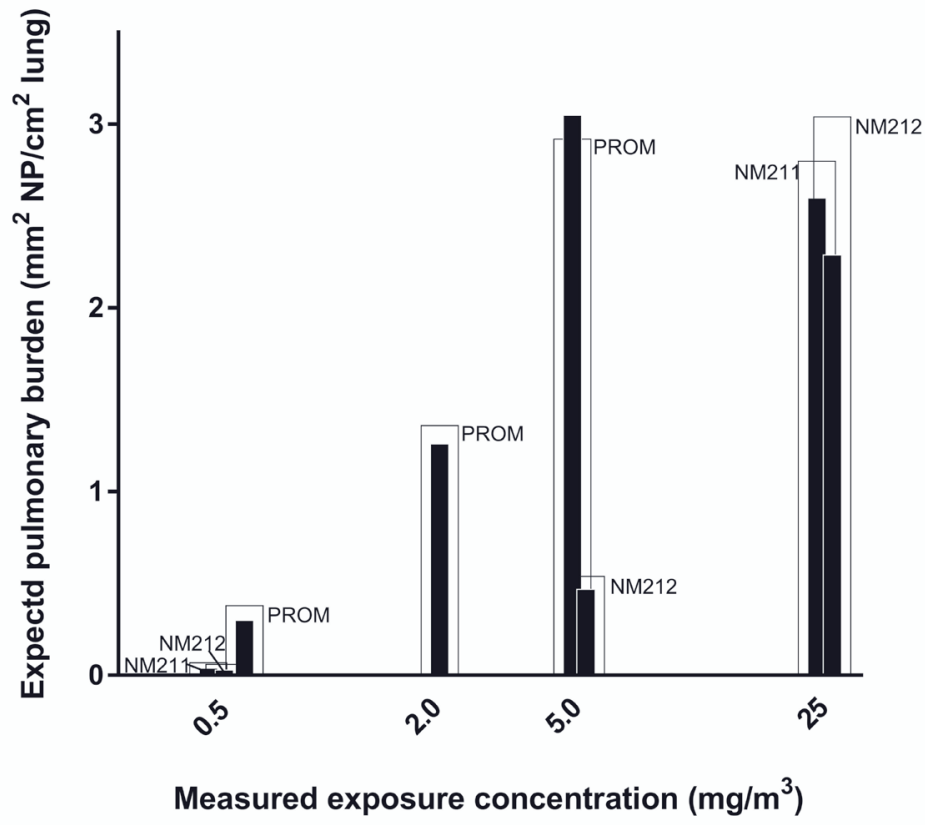

Figure 6: Expected pulmonary burden plotted against the measured exposure concentration in rats after 5 days of exposure to various $\mathrm{CeO}_{2}$ NPs. Each bar represents the expected pulmonary burden either 1 day (white bars) or 3 weeks (black bars) after exposure to a specific exposure concentration of NM211, NM212 or PROM CeO ${ }_{2} \mathrm{NPs}_{\text {. }}$

Besides differences in toxicokinetics of $\mathrm{CeO}_{2} \mathrm{NPs}$ (resulting in differences in LB and PB), differences in the toxicodynamics (i.e. toxic potency) of the different nanoforms of $\mathrm{CeO}_{2}$ may account for the observed differences in toxicity. Immediately after exposure (day 0) more severe changes in BALF parameters and lung histopathology were observed at lower exposure concentrations, LB and PB levels of NM211 and NM212 compared to PROM CeO NPs (Table 5). This indicates that PROM CeO ${ }_{2}$ NPs are less toxic than NM211 and NM212 when expressed as external concentration ( $\mathrm{mg} \mathrm{NP} / \mathrm{m}^{3}$ air), internal mass dose ( $\mu \mathrm{g} \mathrm{NP/g}$ lung), or internal surface area dose $\left(\mathrm{mm}^{2} \mathrm{NP} / \mathrm{cm}^{2}\right.$ lung). Three weeks after exposure, more severe changes in BALF parameters and lung histopathology were observed after exposure to PROM CeO $\mathrm{NPs}_{2}$ compared to $\mathrm{NM} 212$ at certain exposure concentrations (e.g. $5 \mathrm{mg}$ / $\mathrm{m}^{3}$ ) or LBs (60-70 $\mu \mathrm{g} \mathrm{NP/g} \mathrm{lung).} \mathrm{However,} \mathrm{similar} \mathrm{effects} \mathrm{on} \mathrm{BALF} \mathrm{parameters} \mathrm{and} \mathrm{lung}$ histopathology were observed at similar PB levels for all different nanoforms, indicating a similar toxic potency of all nanoforms when the dose is expressed as internal surface area ( $\mathrm{mm}^{2} \mathrm{NP} / \mathrm{cm}^{2}$ lung). 


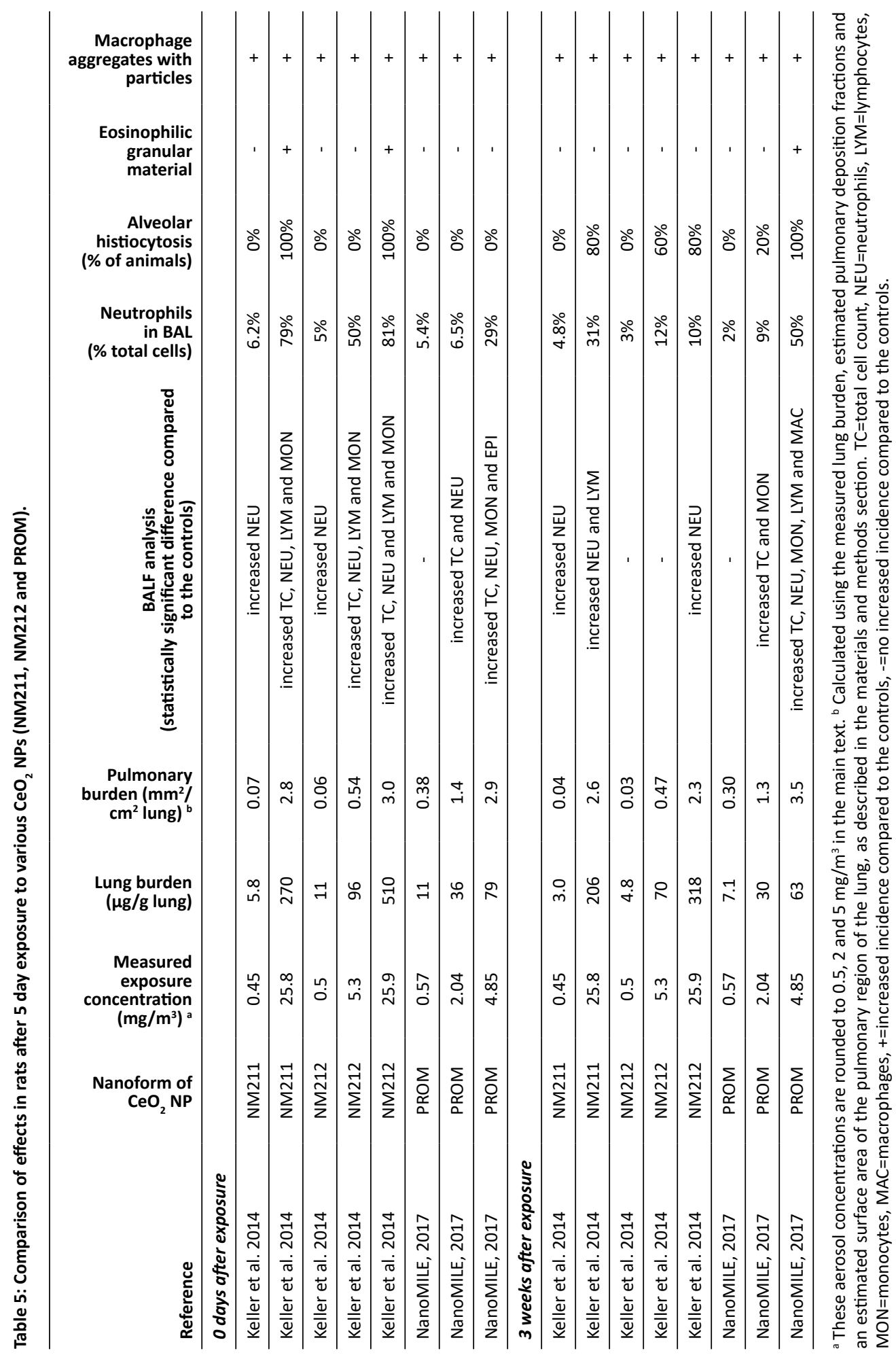



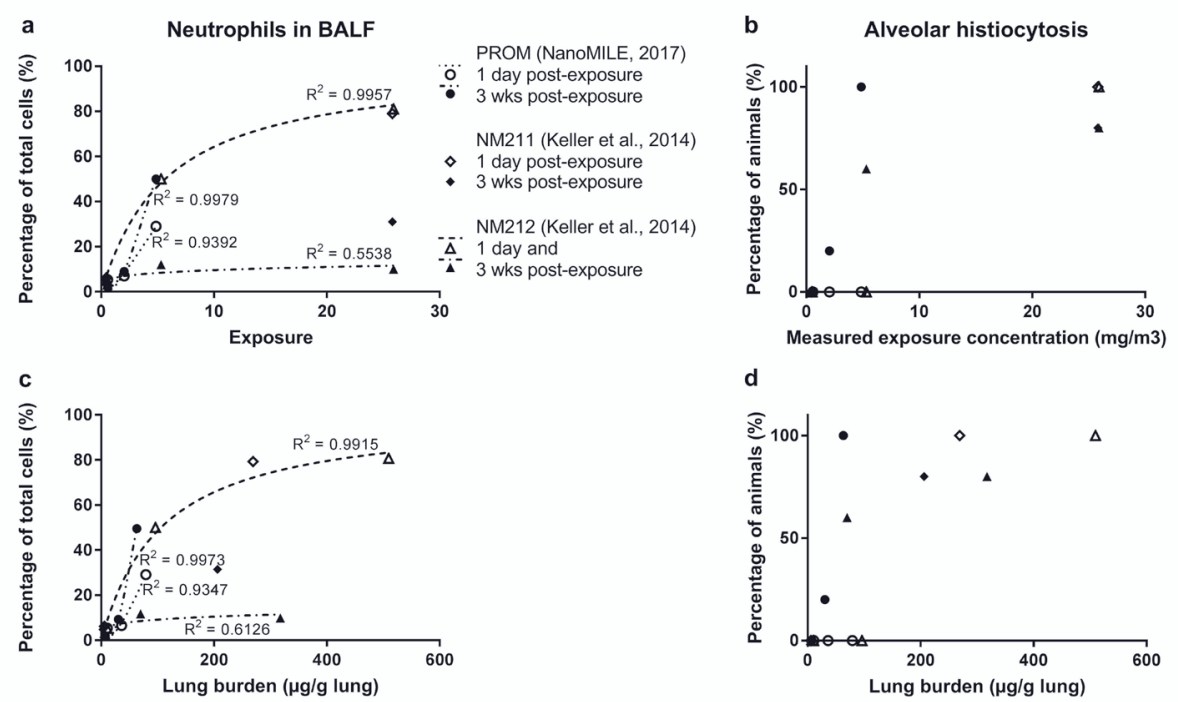

d
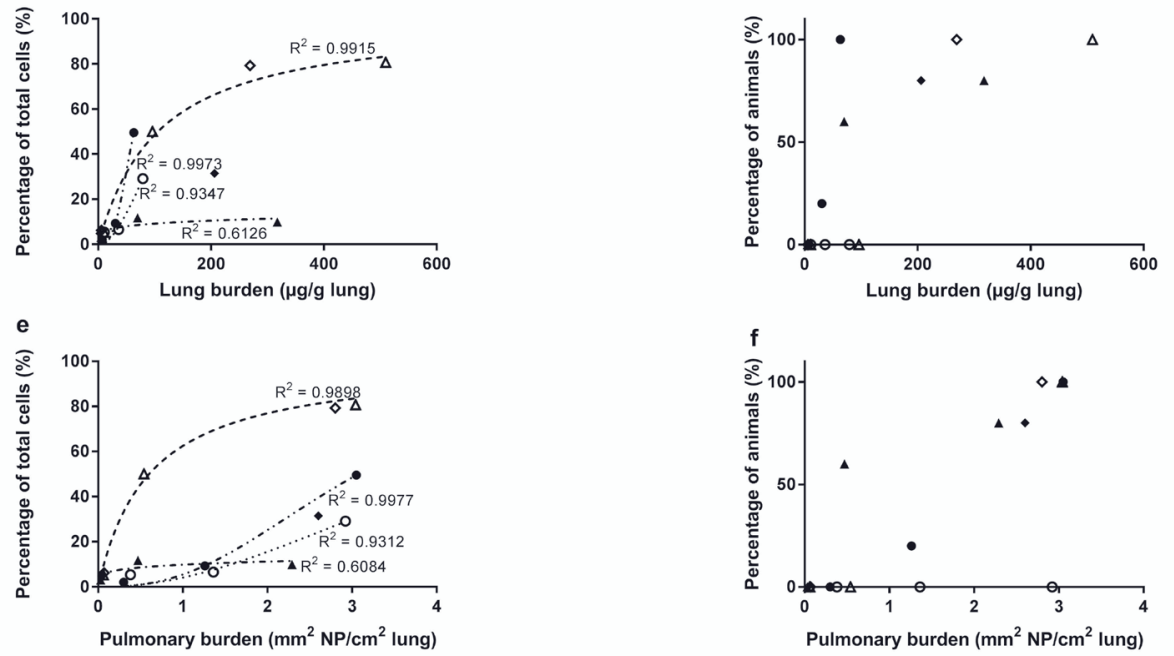

Figure 7: The percentage of neutrophils in the $\operatorname{BALF}(a, c$, and e) and the percentage of animals with alveolar histiocytosis (b, d, and f) after 5 days of exposure of rats to various $\mathrm{CeO}_{2}$ NPs (NM211, NM212 and PROM) plotted against the measured exposure concentration ( $\mathrm{mg} \mathrm{NP} / \mathrm{m}^{3}$ air) (a and b), lung burden ( $\mu \mathrm{g} \mathrm{NP/g} \mathrm{lung)} \mathrm{(c} \mathrm{and} \mathrm{d)} \mathrm{and}$ expected pulmonary burden ( $\mathrm{mm}^{2} \mathrm{NP} / \mathrm{cm}^{2}$ lung) (e and f).

When expressed as exposure concentration (mg NP/m $/ \mathrm{m}^{3}$ air) or LB ( $\mu \mathrm{g} \mathrm{NP/g}$ lung) the dose response curves of the percentage of neutrophils in the BALF are steeper for $\mathrm{PROM} \mathrm{CeO}_{2}$ NPs than for NM212 (see Figure 7a and c), indicating PROM CeO $\mathrm{NPs}_{2}$ are more toxic than NM212 when the dose is expressed as external concentration or internal mass dose. Conversely, when expressed as PB $\left(\mathrm{mm}^{2} \mathrm{NP} / \mathrm{cm}^{2}\right.$ lung) the dose response curves are steeper for NM212 compared to PROM CeO $\mathrm{NPs}_{2} 1$ day post-exposure (see Figure 7e), indicating $\mathrm{NM} 212$ is more acutely toxic than PROM CeO $\mathrm{NPs}_{2}$ when the dose is expressed as internal surface area. Non-linear curve fitting did not result in a good fit for NM212 at 3 weeks postexposure $\left(R^{2}=0.61\right)$, which makes the comparison between the different nanoforms of $\mathrm{CeO}_{2}$ based on dose response curves of the percentage of neutrophils in the BALF 3 weeks postexposure difficult.

The observed differences between the different nanoforms of $\mathrm{CeO}_{2}$ may reflect differences in physicochemical characteristics such as size, shape, surface chemistry (reactivity and charge), dissolution and hydrophobicity of the different nanoforms. Although there are no 
studies available in which the cytotoxicity of the NM212, NM211 and PROM are compared in the same in vitro assays, previous studies have shown that differences in physicochemical properties such as (primary) particles size, surface reactivity and shape may lead to differences in (cyto)toxicity.

Previous studies indicate that smaller particles, aggregates, agglomerates and aerosols result in higher deposition levels in the deep lung and slower clearance rates than larger particles (Braakhuis et al. 2014; Peng et al. 2014). In addition, smaller particles have a relatively large surface area (for an equivalent mass) available for interaction with the body, and potentially can more easily cross barriers or be taken up by macrophages (Nel et al. 2006; Geiser 2010; Arts et al. 2015; Bakand and Hayes 2016). These differences in toxicokinetics and toxicodynamics generally lead to smaller particles having a more potent pulmonary inflammation (acute and chronic) after inhalation. However, differences in dose response for smaller $\mathrm{TiO}_{2}$ and $\mathrm{Ag}$ NPs were found to disappear when the dose was expressed as surface area instead of mass (Oberdorster et al. 2005; Braakhuis et al. 2014). This is in contrast to the toxicity observed here immediately after exposure to $\mathrm{CeO}_{2} \mathrm{NPs}$, which suggests that the nanoforms of $\mathrm{CeO}_{2}$ with the smallest primary particle size (PROM) are less toxic compared to those with larger primary particle sizes and a similar or larger aggregate/ agglomerate size (NM211 and NM212). However, the toxicity observed here 3 weeks postexposure to $\mathrm{CeO}_{2} \mathrm{NPs}$ seems to confirm the findings of Oberdorster and Braakhuis, since the observed differences in toxicity between the nanoforms of $\mathrm{CeO}_{2} \mathrm{NP}$ with different primary particle and aggregate/agglomerate sizes seem to decrease when the dose is expressed as surface area ( $\mathrm{mm}^{2} \mathrm{NP} / \mathrm{cm}^{2}$ lung) instead of mass ( $\mathrm{mg} \mathrm{NP} / \mathrm{m}^{3}$ air or $\mu \mathrm{g} \mathrm{NP/g} \mathrm{lung).}$

Besides size, surface reactivity may also influence toxicity (Warheit et al. 2007; Braakhuis et al. 2014; Peng et al. 2014; Arts et al. 2015; Bakand and Hayes 2016). NPs with a higher surface reactivity are generally able to generate more reactive oxygen species (ROS) that may lead to oxidative stress and inflammatory responses. $\mathrm{CeO}_{2} \mathrm{NPs}$ have been shown to both induce ROS and oxidative stress (Lin et al. 2006; Park et al. 2008; Eom and Choi 2009) as well as protect against oxidant-induced effects (Xia et al. 2008; Celardo et al. 2011). Gandon et al. (2017) showed that NM212 and NM211 had similar reactivity in the Ferric Reduction Ability of Serum (FRAS) assay when the dose was expressed as NM surface area. This is in line with our findings showing a similar toxicity of NM212 and NM211 at similar external and internal concentrations. The reactivity PROM $\mathrm{CeO}_{2} \mathrm{NPs}$ is slightly higher than that of NM212 and NM211 (see Table S5). Although, this increasing reactivity with decreasing size is consistent with the increase of $\mathrm{Ce}^{3+}$ atoms on the surface of smaller particles, this higher reactivity is not in line with our findings showing that PROM $\mathrm{CeO}_{2} \mathrm{NPs}$ are less toxic compared to NM212 and NM211 at similar external and internal concentrations.

Previous studies show contradicting results with respect to the influence of particle shape on the toxicity of $\mathrm{CeO}_{2}$ NPs. Wang et al. (2015) showed that cube-like and octahedron-like 
$\mathrm{CeO}_{2} \mathrm{NPs}$ induced higher cytotoxicity and lower anti-oxidative properties compared to rodlike $\mathrm{CeO}_{2}$ NPs in HepG2 cells. Forest et al. (2017) on the other hand showed that rod-like $\mathrm{CeO}_{2}$ NPs produced significantly and dose-dependently enhanced pro-inflammatory and cytotoxicity responses in RAW264.7 cells, that were not observed after exposure to cubic/ octahedral NPs. Our findings cannot be compared to the results of these previous studies, as they didn't include cubic, octahedral or rod-like $\mathrm{CeO}_{2}$ NPs. TEM images of the nanoforms used in the studies discussed here showed that the nanoform with the least toxic potency right after exposure (PROM) was spherical (Dekkers et al. 2017). Near-spherical NM211 and polyhedral shaped NM212 NPs showed similar toxicity. Because these three nanoforms differ in more than one physicochemical characteristic (Table S5), it is difficult to determine the influence of each individual characteristic on the observed toxicity.

\section{Summary and Conclusion}

In the present study we have used data from several inhalation studies in which mice or rats were exposed to various nanoforms of $\mathrm{CeO}_{2}$ to investigate to what extent the observed differences in toxicity between various studies can be explained by differences in lung deposition, species susceptibility and/or physicochemical characteristics of the tested nanoforms. Considerable differences in pulmonary response were observed between mice and rats (Table 4) and between the various $\mathrm{CeO}_{2}$ nanoforms tested (Table 5). Our evaluations demonstrate that the external exposure concentration, and the NP size and chemical composition $\left(\mathrm{CeO}_{2}\right)$ alone cannot fully explain the observed differences in health effects. The level at which an adverse effect starts to develop is also dependent on the internal dose, animal species and the specific nanoform. We have shown that rats are more sensitive than mice based on both external and internal concentrations. These differences do not result purely from differences in the delivered and retained doses (expressed in particle mass as well as surface area). In addition, the different nanoforms showed differences in toxic potency. Particle size is highly important to this response, but also other physicochemical properties of the $\mathrm{CeO}_{2} \mathrm{NPs}$, such as surface reactivity and surface shape, may influence the toxicity of the nanoform. Based on these findings we conclude that the observed differences in toxicity of the $\mathrm{CeO}_{2} \mathrm{NPs}$ in various inhalation studies can be explained by differences in kinetics and susceptibility of rats compared to mice and differences in size as well as other physicochemical characteristics of the $\mathrm{CeO}_{2} \mathrm{NPs}$. It remains to be determined to what extent these findings can be generalized to other poorly soluble nanomaterials.

\section{Acknowledgements}

The authors would like to thank Dr. Wendel Wohlleben and Kai Werle for the reactivity analysis using the Ferric Reduction Ability of Serum (FRAS) assay. This work was supported by the European Union's Seventh Framework Programme for research, technology development and demonstration under grant agreement $n^{\circ} 310451$ (NanoMILE) and the Netherlands Food and Consumer Product Safety Authority (NVWA). 


\section{References}

Aalapati S, Ganapathy S, Manapuram S, Anumolu G, Prakya BM. 2014. Toxicity and bio-accumulation of inhaled cerium oxide nanoparticles in CD1 mice. Nanotoxicology. 8(7):786-798.

Arts JH, Hadi M, Irfan MA, Keene AM, Kreiling R, Lyon D, Maier M, Michel K, Petry T, Sauer UG et al. 2015. A decision-making framework for the grouping and testing of nanomaterials (DF4nanoGrouping). Regul Toxicol Pharmacol. 71(2 Suppl):S1-27.

Arts JH, Irfan MA, Keene AM, Kreiling R, Lyon D, Maier M, Michel K, Neubauer N, Petry T, Sauer UG et al. 2016. Case studies putting the decision-making framework for the grouping and testing of nanomaterials (DF4nanoGrouping) into practice. Regul Toxicol Pharmacol. 76:234-261.

Bakand S, Hayes A. 2016. Toxicological Considerations, Toxicity Assessment, and Risk Management of Inhaled Nanoparticles. Int J Mol Sci. 17(6).

Bermudez E, Mangum JB, Wong BA, Asgharian B, Hext PM, Warheit DB, Everitt JI. 2004. Pulmonary responses of mice, rats, and hamsters to subchronic inhalation of ultrafine titanium dioxide particles. Toxicol Sci. 77(2):347-357.

Borm P, Cassee FR, Oberdorster G. 2015. Lung particle overload: old school -new insights? Part Fibre Toxicol. 12:10.

Braakhuis HM, Cassee FR, Fokkens PH, de la Fonteyne LJ, Oomen AG, Krystek P, de Jong WH, van Loveren $\mathrm{H}$, Park MV. 2016. Identification of the appropriate dose metric for pulmonary inflammation of silver nanoparticles in an inhalation toxicity study. Nanotoxicology. 10(1):63-73.

Braakhuis HM, Park MV, Gosens I, De Jong WH, Cassee FR. 2014. Physicochemical characteristics of nanomaterials that affect pulmonary inflammation. Part Fibre Toxicol. 11:18.

Buckley A, Warren J, Hodgson A, Marczylo T, Ignatyev K, Guo C, Smith R. 2017. Slow lung clearance and limited translocation of four sizes of inhaled iridium nanoparticles. Part Fibre Toxicol. 14(1):5.

Celardo I, De Nicola M, Mandoli C, Pedersen JZ, Traversa E, Ghibelli L. 2011. Ce(3)+ ions determine redox-dependent anti-apoptotic effect of cerium oxide nanoparticles. ACS Nano. 5(6):4537-4549.

Dekkers S, Miller MR, Schins RPF, Romer I, Russ M, Vandebriel RJ, Lynch I, Belinga-Desaunay MF, ValsamiJones $\mathrm{E}$, Connell SP et al. 2017. The effect of zirconium doping of cerium dioxide nanoparticles on pulmonary and cardiovascular toxicity and biodistribution in mice after inhalation. Nanotoxicology 11:794-808.

Dekkers S, Oomen AG, Bleeker EA, Vandebriel RJ, Micheletti C, Cabellos J, Janer G, Fuentes N, VazquezCampos S, Borges $T$ et al. 2016. Towards a nanospecific approach for risk assessment. Regul Toxicol Pharmacol. 80:46-59.

EC. 2017. Draft regulation Amendments of the Annexes to REACH for registration of nanomatereials. COMMISSION REGULATION (EU) .../... amending Regulation (EC) No 1907/2006 of the European Parliament and of the Council on the Registration, Evaluation, Authorisation and Restriction of Chemicals (REACH) as regards Annexes I, III,VI, VII, VIII, IX, X, XI, and XII to address nanoforms of substances. In: Environment D-Gf, editor.

ECHA. 2017. How to prepare registration dossier that cover nanoforms: best practices. Helsinki, Finland: European Chemicals Agency. ECHA-17-G-13-EN. 
Elder A, Gelein R, Finkelstein JN, Driscoll KE, Harkema J, Oberdorster G. 2005. Effects of subchronically inhaled carbon black in three species. I. Retention kinetics, lung inflammation, and histopathology. Toxicol Sci. 88(2):614-629.

Eom HJ, Choi J. 2009. Oxidative stress of $\mathrm{CeO} 2$ nanoparticles via p38-Nrf-2 signaling pathway in human bronchial epithelial cell, Beas-2B. Toxicol Lett. 187(2):77-83.

Forest V, Leclerc L, Hochepied JF, Trouve A, Sarry G, Pourchez J. 2017. Impact of cerium oxide nanoparticles shape on their in vitro cellular toxicity. Toxicol In Vitro. 38:136-141.

Gandon A, Werle K, Neubauer N, Wohlleben W. 2017. Surface reactivity measurements as required for grouping and read-across: An advanced FRAS protocol. J Phys. Conf Ser 838.

Geiser M. 2010. Update on macrophage clearance of inhaled micro- and nanoparticles. J Aerosol Med Pulm Drug Deliv. 23(4):207-217.

Geraets L, Oomen AG, Schroeter JD, Coleman VA, Cassee FR. 2012. Tissue distribution of inhaled micro- and nano-sized cerium oxide particles in rats: results from a 28-day exposure study. Toxicol Sci. 127(2):463-473.

Gosens I, Mathijssen LE, Bokkers BG, Muijser H, Cassee FR. 2014. Comparative hazard identification of nano- and micro-sized cerium oxide particles based on 28-day inhalation studies in rats. Nanotoxicology. 8(6):643-653.

Greim H, Ziegler-Skylakakis K. 2007. Risk assessment for biopersistent granular particles. Inhal Toxicol. 19 Suppl 1:199-204.

Han SG, Lee JS, Ahn K, Kim YS, Kim JK, Lee JH, Shin JH, Jeon KS, Cho WS, Song NW et al. 2015. Sizedependent clearance of gold nanoparticles from lungs of Sprague-Dawley rats after short-term inhalation exposure. Arch Toxicol. 89(7):1083-1094.

Keller J, Wohlleben W, Ma-Hock L, Strauss V, Groters S, Kuttler K, Wiench K, Herden C, Oberdorster G, van Ravenzwaay B et al. 2014. Time course of lung retention and toxicity of inhaled particles: short-term exposure to nano-Ceria. Arch Toxicol. 88(11):2033-2059.

Kreyling WG, Semmler-Behnke M, Takenaka S, Moller W. 2013. Differences in the biokinetics of inhaled nano- versus micrometer-sized particles. Acc Chem Res. 46(3):714-722.

Kuempel ED, Sweeney LM, Morris JB, Jarabek AM. 2015. Advances in Inhalation Dosimetry Models and Methods for Occupational Risk Assessment and Exposure Limit Derivation. J Occup Environ Hyg. 12 Suppl 1:S18-40.

Landsiedel R, Ma-Hock L, Hofmann T, Wiemann M, Strauss V, Treumann S, Wohlleben W, Groters S, Wiench K, van Ravenzwaay B. 2014. Application of short-term inhalation studies to assess the inhalation toxicity of nanomaterials. Part Fibre Toxicol. 11:16.

Lin W, Huang YW, Zhou XD, Ma Y. 2006. Toxicity of cerium oxide nanoparticles in human lung cancer cells. Int J Toxicol. 25(6):451-457.

NanoMILE. 2017. D 7.2 Molecular mechanisms of toxicity of Manufactured Nanomaterials (MNMs) in vivo: Identification of molecular mechanisms of toxicity in vivo (e.g. inhalation toxicity, myography, blood thrombogenicity) with emphasis on effects of surface coating and charge.: NRCWE, RIVM, UEDIN, BASF SE and IUF. 
Nel A, Xia T, Madler L, Li N. 2006. Toxic potential of materials at the nanolevel. Science. 311(5761):622627.

Oberdorster G, Oberdorster E, Oberdorster J. 2005. Nanotoxicology: an emerging discipline evolving from studies of ultrafine particles. Environ Health Perspect. 113(7):823-839.

Oomen AG, Bleeker EA, Bos PM, van Broekhuizen F, Gottardo S, Groenewold M, Hristozov D, HundRinke K, Irfan MA, Marcomini A et al. 2015. Grouping and Read-Across Approaches for Risk Assessment of Nanomaterials. Int J Environ Res Public Health. 12(10):13415-13434.

Park EJ, Choi J, Park YK, Park K. 2008. Oxidative stress induced by cerium oxide nanoparticles in cultured BEAS-2B cells. Toxicology. 245(1-2):90-100.

Pauluhn J. 2017. Kinetic modeling of the retention and fate of inhaled cerium oxide nanoparticles in rats: The cumulative displacement volume of agglomerates determines the outcome. Regul Toxicol Pharmacol. 86:319-331.

Peng L, He X, Zhang P, Zhang J, Li Y, Zhang J, Ma Y, Ding Y, Wu Z, Chai Z et al. 2014. Comparative pulmonary toxicity of two ceria nanoparticles with the same primary size. Int J Mol Sci. 15(4):6072-6085.

Semmler M, Seitz J, Erbe F, Mayer P, Heyder J, Oberdorster G, Kreyling WG. 2004. Long-term clearance kinetics of inhaled ultrafine insoluble iridium particles from the rat lung, including transient translocation into secondary organs. Inhal Toxicol. 16(6-7):453-459.

Stone V, Pozzi-Mucelli S, Tran L, Aschberger K, Sabella S, Vogel U, Poland C, Balharry D, Fernandes T, Gottardo S et al. 2014. ITS-NANO--prioritising nanosafety research to develop a stakeholder driven intelligent testing strategy. Part Fibre Toxicol. 11:9.

Wang L, Ai W, Zhai Y, Li H, Zhou K, Chen H. 2015. Effects of Nano-CeO(2) with Different Nanocrystal Morphologies on Cytotoxicity in HepG2 Cells. Int J Environ Res Public Health. 12(9):10806-10819.

Warheit DB, Kreiling R, Levy LS. 2016. Relevance of the rat lung tumor response to particle overload for human risk assessment-Update and interpretation of new data since ILSI 2000. Toxicology. 374:42-59.

Warheit DB, Webb TR, Colvin VL, Reed KL, Sayes CM. 2007. Pulmonary bioassay studies with nanoscale and fine-quartz particles in rats: toxicity is not dependent upon particle size but on surface characteristics. Toxicol Sci. 95(1):270-280.

Wiemann M, Vennemann A, Sauer UG, Wiench K, Ma-Hock L, Landsiedel R. 2016. An in vitro alveolar macrophage assay for predicting the short-term inhalation toxicity of nanomaterials [journal article]. Journal of Nanobiotechnology. 14(1):16.

Xia T, Kovochich M, Liong M, Madler L, Gilbert B, Shi H, Yeh JI, Zink JI, Nel AE. 2008. Comparison of the mechanism of toxicity of zinc oxide and cerium oxide nanoparticles based on dissolution and oxidative stress properties. ACS Nano. 2(10):2121-2134.

Zhang H, Ji Z, Xia T, Meng H, Low-Kam C, Liu R, Pokhrel S, Lin S, Wang X, Liao YP et al. 2012. Use of metal oxide nanoparticle band gap to develop a predictive paradigm for oxidative stress and acute pulmonary inflammation. ACS Nano. 6(5):4349-4368. 


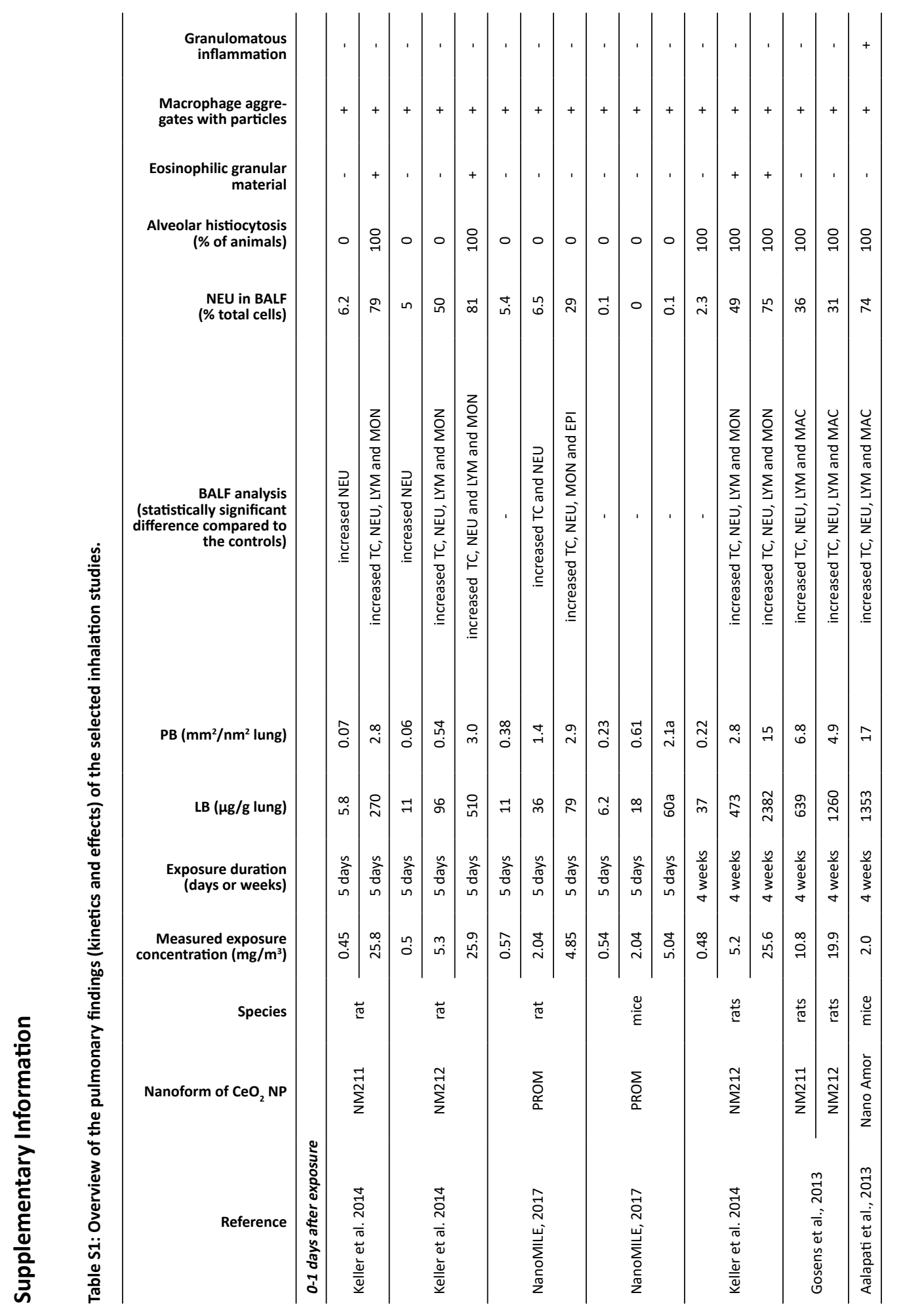




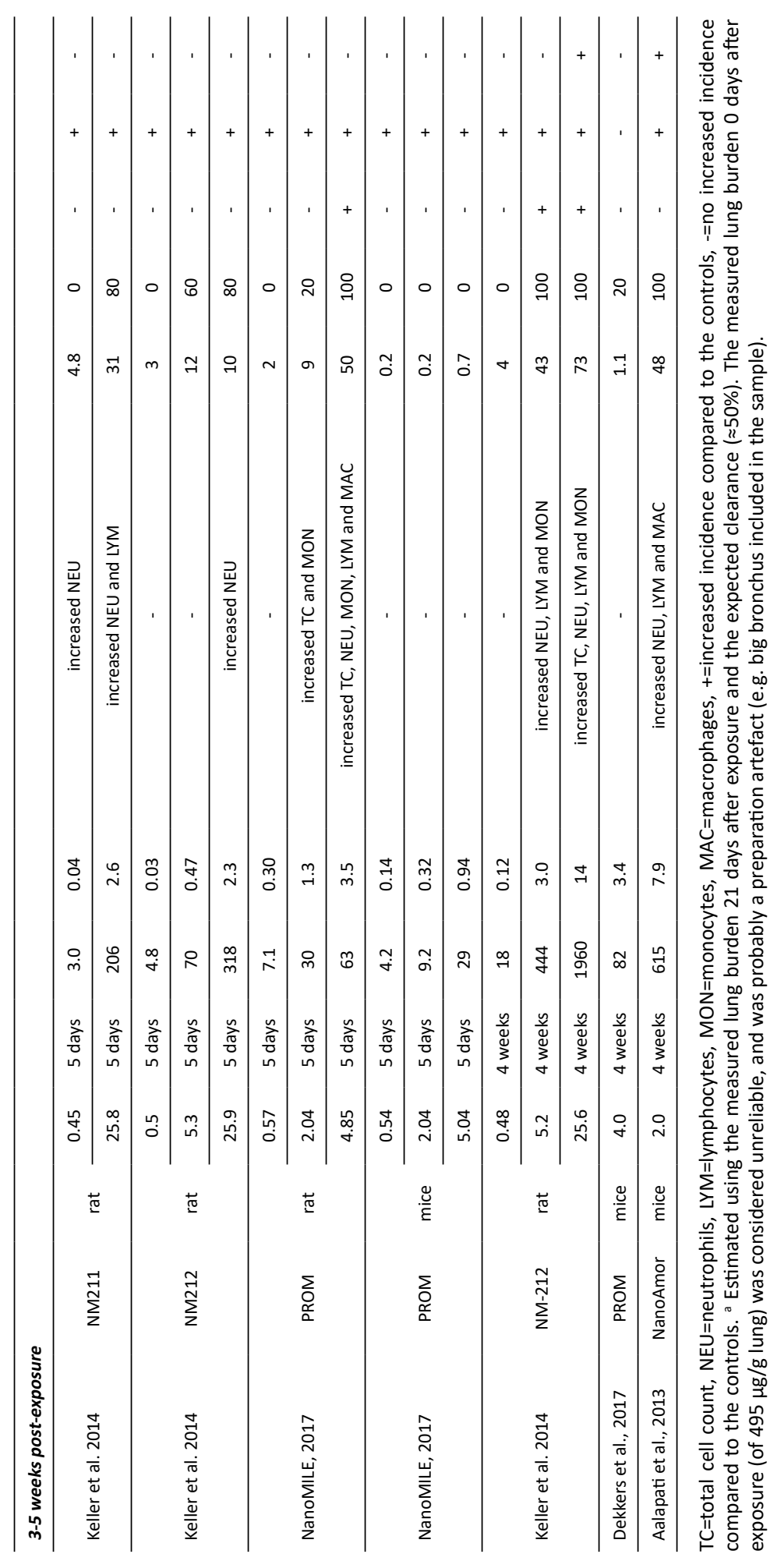




\section{Annex S2: Summary of the short term inhalation studies with PROM CeO $\mathrm{NPs}_{2}$ Study design and exposure}

The study design for these additional studies with PROM $\mathrm{CeO}_{2}$ NPs followed that of previously performed 5 day inhalation studies with NM212, but used nose-only exposure instead of whole body exposure, to allow better comparison with the 4 week inhalation study with PROM CeO ${ }_{2}$ NPs in mice as reported previously (Dekker et al., 2017). Briefly, male Wistar rats and male $\mathrm{C} 57 \mathrm{BL} / 6$ mice were nose-only exposed to target concentrations of 0.5 , 2 and $5 \mathrm{mg} / \mathrm{m}^{3} \mathrm{CeO}_{2} \mathrm{NPs}$ in respirable aerosols for 6 hours per day, on 5 consecutive days. The actual exposure concentrations were similar for rats $\left(0.57,2.04\right.$ and $\left.4.85 \mathrm{mg} / \mathrm{m}^{3}\right)$ and mice $\left(0.54,2.04\right.$ and $5.04 \mathrm{mg} / \mathrm{m}^{3}$ ) with similar particle size distributions (MMAD between 1.0 and $1.3 \mu \mathrm{m}$ for rats and between 0.9 and $1.2 \mu \mathrm{m}$ for mice; see Table S3). The concurrent control group was exposed to clean air only. During the study, the animals were monitored for mortality and clinical signs of toxicity. Body weights were determined twice weekly. Autopsy was performed and toxicity parameters were evaluated either 24 hours after the last exposure or after a post-exposure recovery period of 3 weeks (see Table S2). Blood was sampled, selected organs were weighed and a broad set of organs and tissues were preserved. The respiratory tract was examined histologically in 5 animals per group. Clinical chemistry parameters, hematology parameters and acute phase proteins were examined in blood of the same 5 animals per group for rats and 3 or 5 additional animals per group for mice. After blood sampling these animals underwent bronchoalveolar lavage of the left lung. Bronchoalveolar lavage fluid (BALF) was examined for cytological and biochemical parameters including selected antigens. Lung burdens were determined in 3 additional animals per group for both rats and mice.

Table S2: Overview of number of animals per autopsy group.

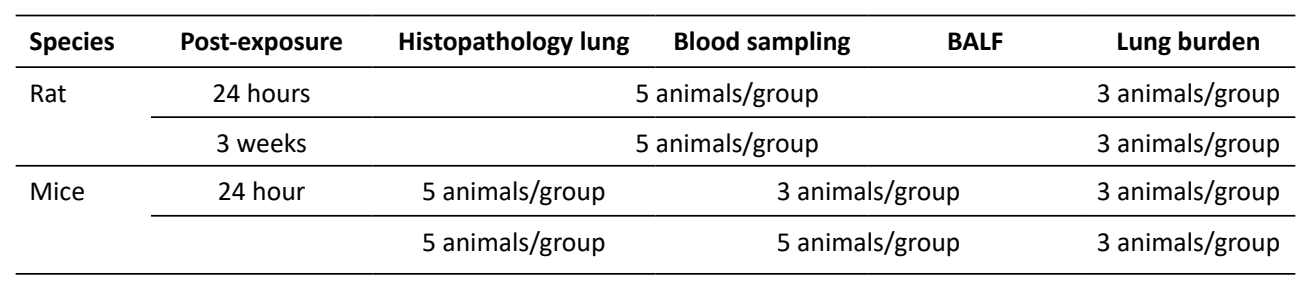




\section{Lung burden}

The results of lung burden measured 24 hours after the exposure and after a 3 week recovery period are shown in Figures S2.1 and S2.2, in rats and mice, respectively.

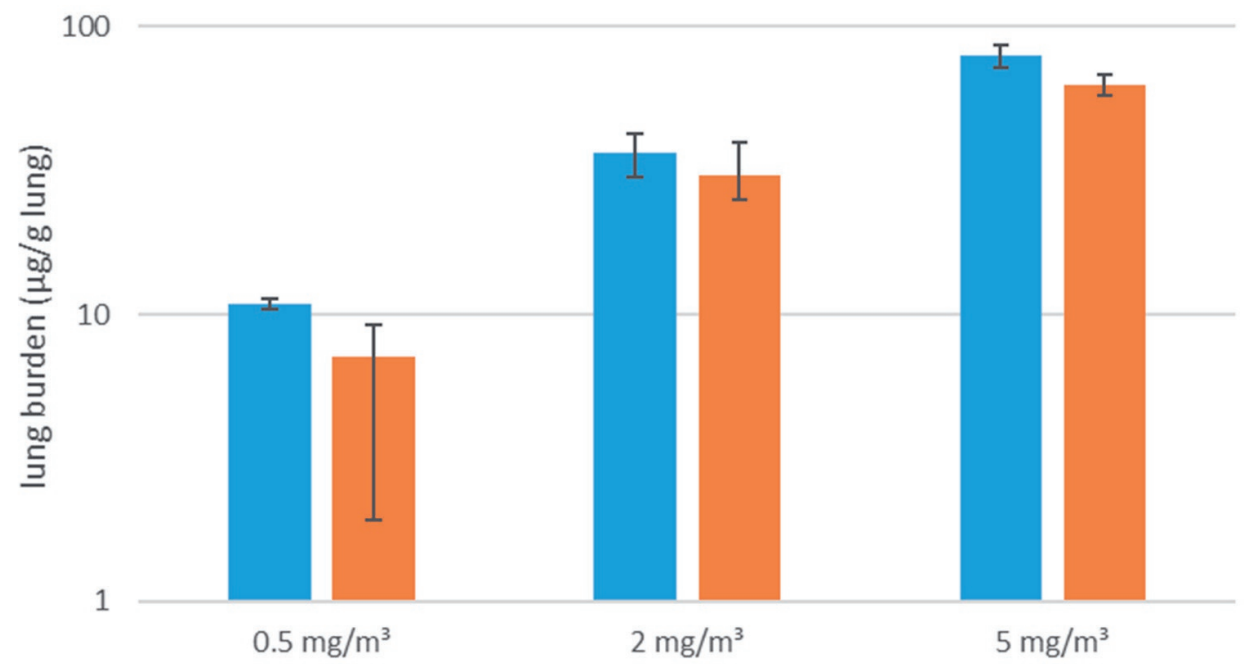

Figure S2.1: Lung burden after 5 days of exposure to $\mathrm{CeO}_{2} \mathrm{NPs} 24$ hours (blue bars) and 3 weeks (orange bars) post-exposure in rats.

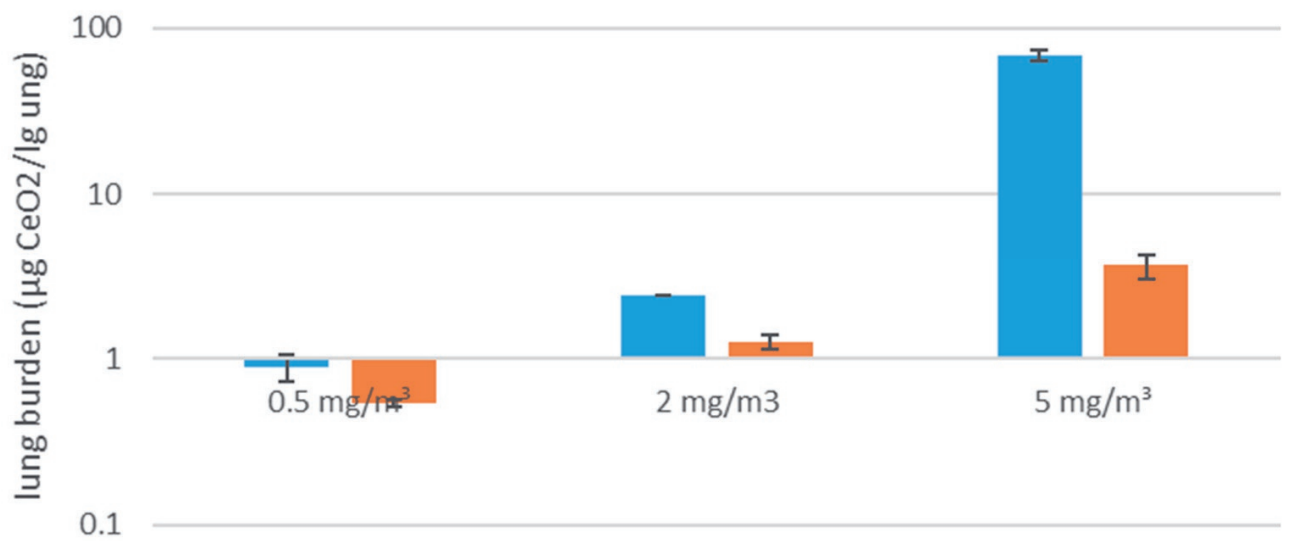

Figure S2.2: Lung burden after 5 days of exposure to $\mathrm{CeO}_{2} \mathrm{NPs} 24$ hours (blue bars) and 3 weeks (orange bar) postexposure in mice. 
Ce could not be detected in the lung of the control animals (detection limit: $0.3 \mu \mathrm{g}$ per sample). In rats a modest clearance was observed in animals exposed to $0.5 \mathrm{mg} / \mathrm{m}^{3} \mathrm{CeO}_{2}$ NPs the after 3 weeks recovery, whereas the clearance of 2 and $5 \mathrm{mg} / \mathrm{m}^{3}$ seemed minimal (Figure S2.1). In mice the lung burden was low (0.9 \& $2.4 \mu \mathrm{g}$ Ce/lung) in animals exposed to $0.5 \mathrm{mg} / \mathrm{m}^{3}$ and $2 \mathrm{mg} / \mathrm{m} \mathrm{CeO}_{2} \mathrm{NPs}$ after exposure, whereas the mean lung burden in mice exposed to $5 \mathrm{mg} / \mathrm{m}^{3} \mathrm{CeO}_{2}$ NPs was extraordinary high ( $\sim 9 \mathrm{\mu g} \mathrm{Ce} / \mathrm{lung}$; Figure S2.2). The high value (of $495 \mathrm{\mu g} / \mathrm{g}$ lung) of mice exposed to $5 \mathrm{mg} / \mathrm{m}^{3} \mathrm{CeO}_{2} \mathrm{NPs}$ on study day 5 (last blue bar) was out of range and probably a preparation artefact (e.g. big bronchus included in the sample). In the mice exposed to 0.5 and $2 \mathrm{mg} / \mathrm{m}^{3} \mathrm{CeO}_{2} \mathrm{NPs}$ a clearance of approximately $50 \%$ was observed within the 3 weeks recovery period. A similar clearance rate was assumed for mice exposed to $5 \mathrm{mg} / \mathrm{m}^{3} \mathrm{CeO}_{2} \mathrm{NPs}$.

\section{Hematology and clinical chemistry}

No treatment-related effects were observed for hematology or clinical chemistry parameters, except for a slight, but statistically significant increase in the absolute neutrophil counts in rats exposed to 2 and $5 \mathrm{mg} / \mathrm{m}^{3} \mathrm{CeO}_{2} \mathrm{NPs}$ after the three weeks recovery period (see NanoMILE Deliverable D7.2), indicating a mild systemic inflammation.

\section{Bronchoalveolar lavage fluid (BALF)}

A moderate increase in neutrophil counts was found in the BALF of rats exposed to 2 or 5 $\mathrm{mg} / \mathrm{m}^{3} \mathrm{CeO}_{2} \mathrm{NPs} 24 \mathrm{~h}$ after exposure (Figure S2.3). This was associated with higher alkaline phosphatase (ALP) activity and cytokine-induced chemoattractant-1 (CINC-1/IL8) levels. ALP is released by neutrophils and CINC- 1 is a cytokine which attracts neutrophils into the lung tissue. Epithelial cell counts in BALF were marginally increased in rats exposed to $5 \mathrm{mg} / \mathrm{m}^{3}$ $\mathrm{CeO}_{2}$ NPs, indicating an effect on the bronchioles. There was also in increased monocyte number and in the cytokines osteopontin and macrophage colony stimulating factor (M-CSF) in rats exposed to $5 \mathrm{mg} / \mathrm{m}^{3} \mathrm{CeO}_{2} \mathrm{NPs}$. Increased monocyte chemoattractant protein-1 (MCP1) in rats exposed to 2 and $5 \mathrm{mg} / \mathrm{m}^{3} \mathrm{CeO}_{2}$ NPs also indicates that more monocytes and macrophages were attracted into the lung. A higher permeability of the capillaries in the alveoli can be assumed because of slightly higher total protein levels in BALF of rats exposed to $5 \mathrm{mg} / \mathrm{m}^{3} \mathrm{CeO}_{2} \mathrm{NPs}$. The enzyme activities of lactate dehydrogenase (LDH) and $\gamma$-glutamyl transferase (GGT) in BALF of rats exposed to 2 and $5 \mathrm{mg} / \mathrm{m}^{3} \mathrm{CeO}_{2} \mathrm{NPs}$ were only marginally, but statistically significantly, increased. 


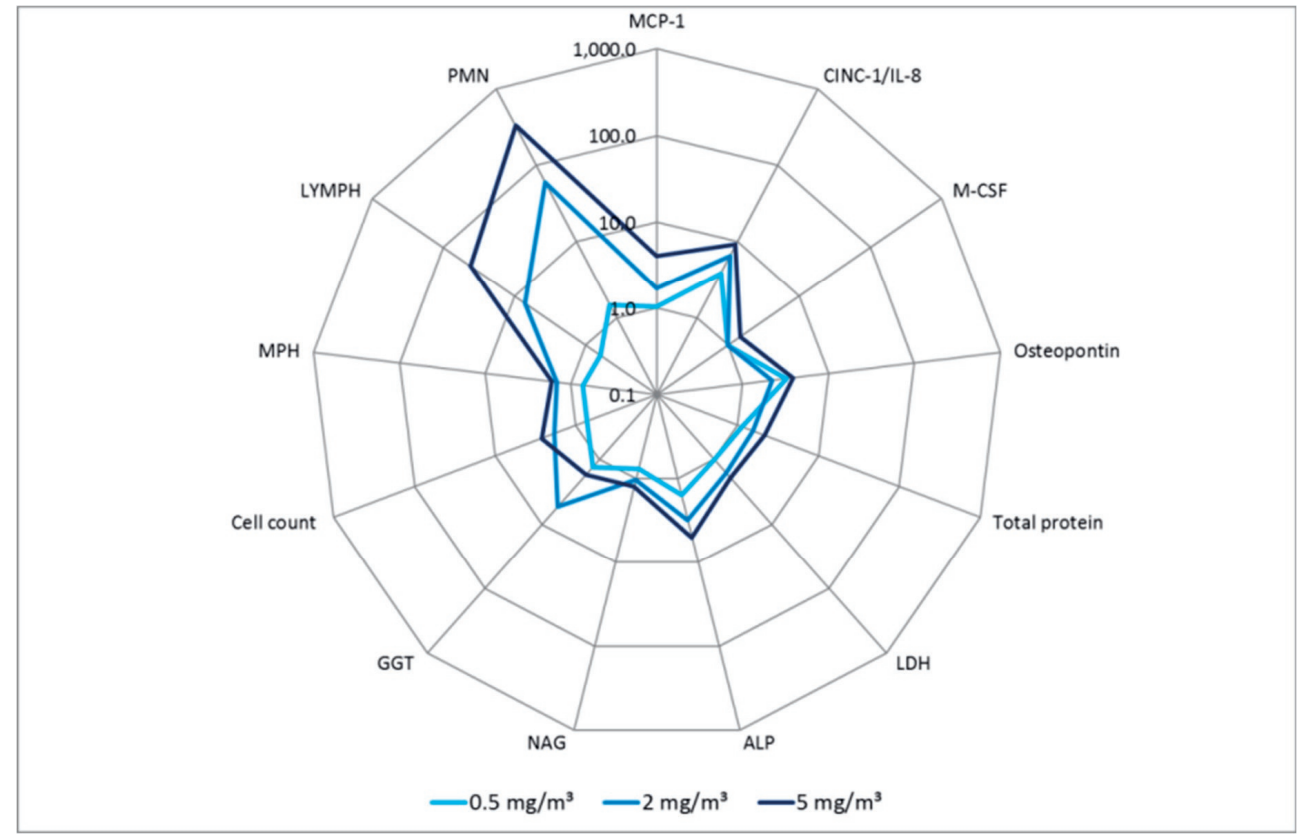

Figure S2.3: Changes in various BALF parameters in rats on study day 5 ( 24 hours after last exposure). Results are presented as $\mathrm{x}$-fold changes compared to concurrent controls. Cell count $=$ total cell count, $\mathrm{MPH}=\mathrm{alveolar}$ macrophage, $\mathrm{LYMPH}=$ lymphocyte, $\mathrm{PMN}=$ polymorphnuclear neutrophilic granulocyte, $\mathrm{GGT}=\mathrm{y}$-Glutamyltransferase; $\mathrm{LDH}=$ Lactate dehydrogenase; $A L P=$ Alkaline phosphatase; NAG = $\beta$-N-Acetyl glucosaminidase, GGT = y-Glutamyl-transferase; $\mathrm{LDH}=$ Lactate dehydrogenase; $\mathrm{ALP}=$ Alkaline phosphatase; $\mathrm{NAG}=\beta-\mathrm{N}-$ Acetyl glucosaminidase, CINC-1/IL-8 = cytokine-induced neutrophil chemoattractant-1; MCP-1 = monocyte chemoattractant protein-1; M-CSF = macrophage colony stimulating factor.

In the BALF, monocyte cell counts were increased in rats exposed to 2 and $5 \mathrm{mg} / \mathrm{m}^{3} \mathrm{CeO}_{2}$ NPs (Figure S2.4). Absolute neutrophil counts were higher in the BALF of rats exposed to 5 $\mathrm{mg} / \mathrm{m}^{3} \mathrm{CeO}_{2} \mathrm{NPs} 3$ weeks post-exposure compared to 24 hours post-exposure. Lymphocyte counts were also increased in rats exposed to $5 \mathrm{mg} / \mathrm{m}^{3} \mathrm{CeO}_{2} \mathrm{NPs}$, indicating an ongoing inflammatory process in the lungs. This was also reflected by high levels of MCP-1 in rats exposed to $5 \mathrm{mg} / \mathrm{m}^{3} \mathrm{CeO}_{2}$ NPs. CINC-1/IL-8 levels in rats exposed to $5 \mathrm{mg} / \mathrm{m}^{3} \mathrm{CeO}_{2}$ NPs were lower compared to 24 hours post-exposure, but were still raised above control levels. The total protein, LDH and GGT levels in BALF of rats exposed to $5 \mathrm{mg} / \mathrm{m}^{3} \mathrm{CeO}_{2} \mathrm{NPs} 3$ weeks postexposure were marginally increased compared to the controls, with levels similar to those 24 hours post-exposure. ALP activity was lower compared to the 24 hours post exposure, but still marginally above control levels. 


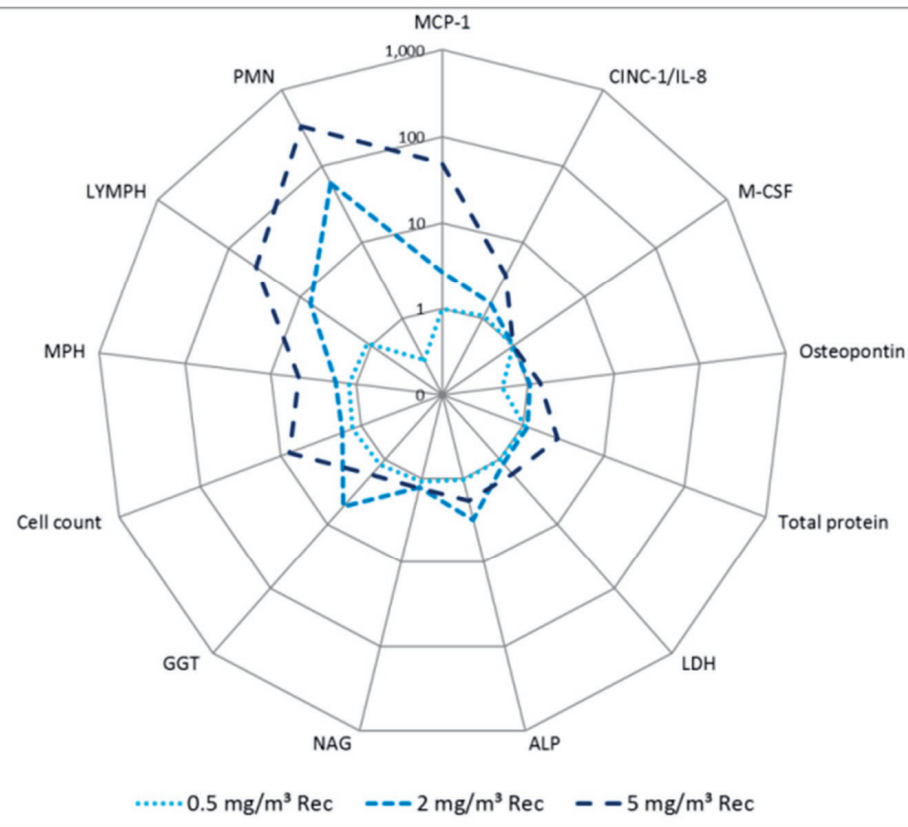

Figure S2.4: Changes in various BALF parameters in rats after a three weeks recovery period on study day 26. Results are presented as $x$-fold changes compared to concurrent controls. Cell count $=$ total cell count, $\mathrm{MPH}=$ alveolar macrophage, $\mathrm{LYMPH}=$ lymphocyte, $\mathrm{PMN}=$ polymorph nuclear neutrophilic granulocyte, GGT = y-Glutamyl-transferase; $L D H=$ Lactate dehydrogenase; $A L P=$ Alkaline phosphatase; NAG = $\beta$-N-Acetyl glucosaminidase, $\mathrm{GGT}=\mathrm{y}$-Glutamyl-transferase; $\mathrm{LDH}=$ Lactate dehydrogenase; $\mathrm{ALP}=$ Alkaline phosphatase; NAG = $\beta-\mathrm{N}-$-Acetyl glucosaminidase, $\mathrm{CINC}-1 / \mathrm{IL}-8$ = cytokine-induced neutrophil chemoattractant-1; MCP-1 = monocyte chemoattractant protein-1; M-CSF = macrophage colony stimulating factor.

No treatment-related changes in cytology parameters, enzyme activities or total protein levels in BALF were observed in mice.

\section{Pathology}

\section{Histopathological finding in the lungs}

In all exposed groups of both rats and mice, characteristic particles, either single or very few, were observed in the cytoplasm of alveolar macrophages (histiocytes) or free in the alveoli. These particles were considered to represent test substance. They occurred in all animals of all treatment groups, and were still present after the recovery period in all rats exposed to $0.5 \mathrm{mg} / \mathrm{m}^{3} \mathrm{CeO}_{2} \mathrm{NPs}$, in 4 rats exposed to $2 \mathrm{mg} / \mathrm{m}^{3} \mathrm{CeO}_{2} \mathrm{NPs}$ and all mice of all exposed groups. In rats, the particles were also seen in the bronchus-associated lymphoid tissue (BALT) or in macrophages in the BALT in two rats exposed to $0.5 \mathrm{mg} / \mathrm{m}^{3} \mathrm{CeO}_{2} \mathrm{NPs}$ and in all rats exposed to 2 and $5 \mathrm{mg} / \mathrm{m}^{3} \mathrm{CeO}_{2}$ NPs. Particles were also present in the BALT after the recovery period in 3 rats exposed to $0.5 \mathrm{mg} / \mathrm{m}^{3} \mathrm{CeO}_{2} \mathrm{NPs}$ and in all rats exposed to 2 and 5 $\mathrm{mg} / \mathrm{m}^{3} \mathrm{CeO}_{2}$ NPs. 
After the recovery period, a minimal alveolar histiocytosis with particles occurred in one rat exposed to $2 \mathrm{mg} / \mathrm{m}^{3} \mathrm{CeO}_{2} \mathrm{NPs}$ and in all rats exposed to $5 \mathrm{mg} / \mathrm{m}^{3} \mathrm{CeO}_{2} \mathrm{NPs}$. In addition, all rats exposed to $5 \mathrm{mg} / \mathrm{m}^{3} \mathrm{CeO}_{2} \mathrm{NPs}$ showed very occasional eosinophilic granular material, most probably debris of degraded macrophages, free in the alveoli.

The occurrence of alveolar histiocytosis with particles in the recovery group in combination with the occurrence of eosinophilic granular material in rats exposed to $5 \mathrm{mg} / \mathrm{m}^{3} \mathrm{CeO}_{2} \mathrm{NPs}$ was regarded to be an indicator of an adverse reaction, whereas the occurrence of particles in single histiocytes or in the BALT in the lungs was considered non-adverse, as there were no signs of any cytotoxicity.

Single or very few particles could be noted within single histiocytes in all exposed mice after the recovery period. The occurrence of these particles in the lungs was considered to be treatment-related, but because there were no signs of an inflammatory response, it was regarded to be non-adverse.

\section{Histopathological findings in the mediastinal and tracheobronchial lymph nodes}

In rats exposed to the $5 \mathrm{mg} / \mathrm{m}^{3} \mathrm{CeO}_{2} \mathrm{NPs}$, single or very few particles were observed in the mediastinal lymph node in one animal and in the tracheobronchial lymph nodes in 3 rats. After the recovery period single or very few particles were seen in the mediastinal lymph node of one rat exposed to $2 \mathrm{mg} / \mathrm{m}^{3} \mathrm{CeO}_{2} \mathrm{NPs}$ and in 4 rats exposed to $5 \mathrm{mg} / \mathrm{m}^{3} \mathrm{CeO}_{2} \mathrm{NPs}$, as well as in the tracheobronchial lymph nodes of 2 rats exposed to $2 \mathrm{mg} / \mathrm{m}^{3} \mathrm{CeO}_{2} \mathrm{NPs}$ and in all rats exposed to $5 \mathrm{mg} / \mathrm{m}^{3} \mathrm{CeO}_{2} \mathrm{NPs}$. In mice, particles were seen free in the lymphoid tissue of the tracheobronchial and mediastinal lymph nodes after the recovery period, and in one mouse exposed to $2 \mathrm{mg} / \mathrm{m}^{3} \mathrm{CeO}_{2} \mathrm{NPs}$ and in one mouse exposed to $5 \mathrm{mg} / \mathrm{m}^{3} \mathrm{CeO}_{2}$ NPs. Because there was no activation or aggregation of macrophages, the occurrence of these particles was regarded to be non-adverse. 


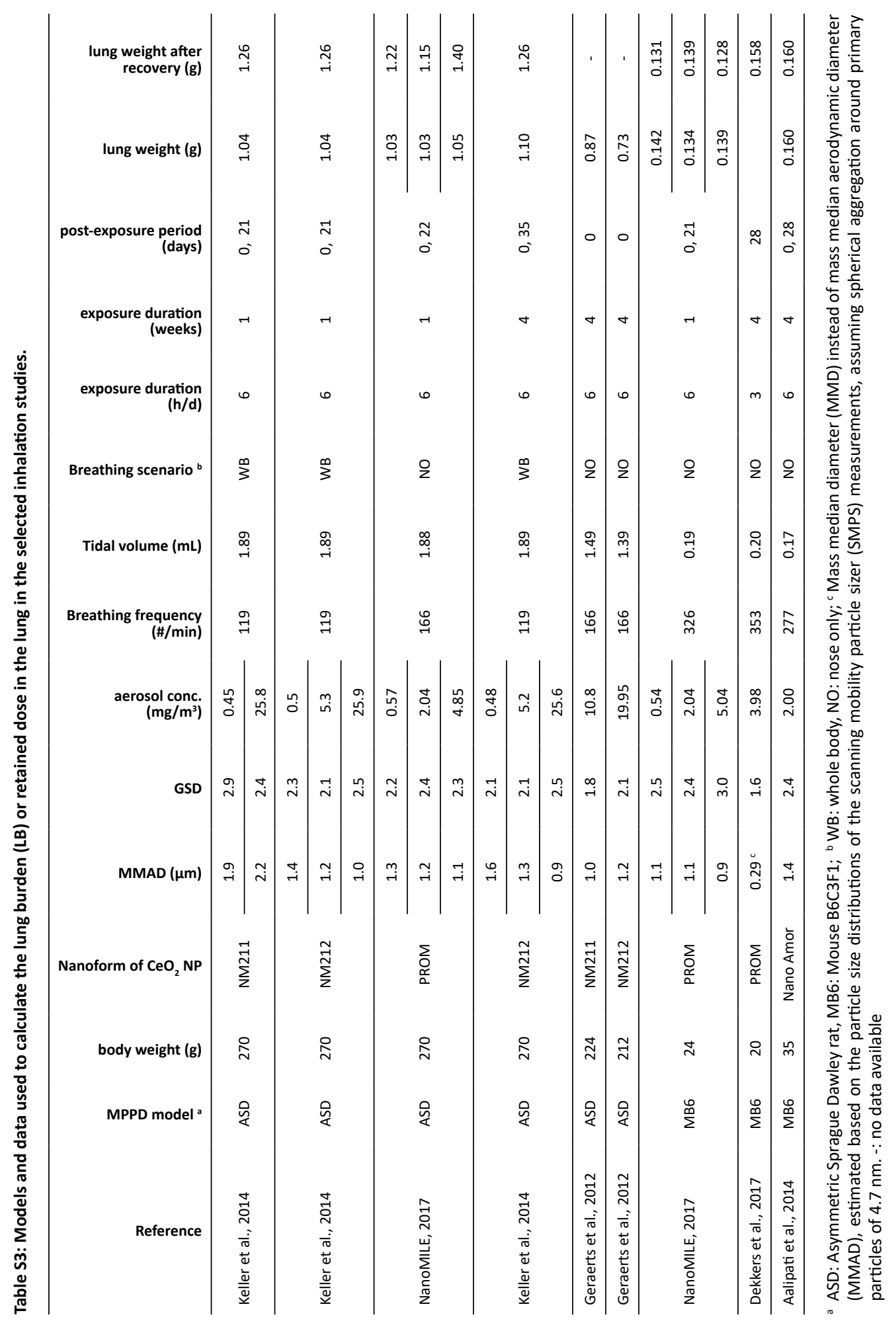




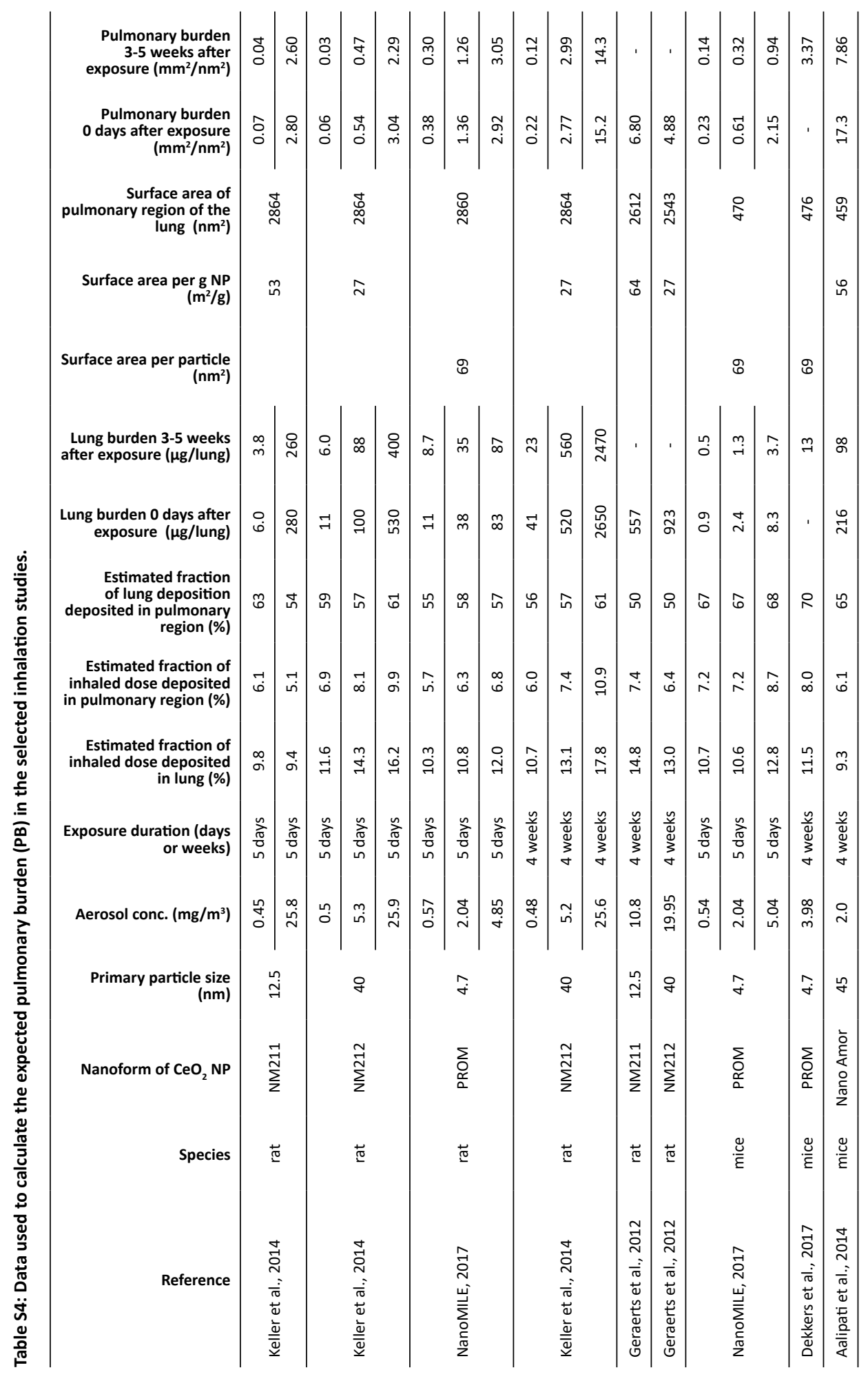


Table S5: Physicochemical characteristics of the $\mathrm{CeO}_{2}$ nanoparticles used in the 5-day inhalation studies in rats.

\begin{tabular}{|c|c|c|c|}
\hline Nanoform of $\mathrm{CeO}_{2} \mathrm{NP} \rightarrow$ & NM211 & NM212 & PROM \\
\hline Shape & near spherical & polyhedral & spherical \\
\hline Primary particle size (nm) & 12.5 & 40 & 4.7 \\
\hline Surface area (BET: $\left.\mathrm{m}^{2} / \mathrm{g}\right)$ & 64 & 27 & - \\
\hline Surface reactivity $\left(\mathrm{nmol} \mathrm{TEU} / \mathrm{m}^{2} \mathrm{NP}\right)^{\mathrm{a}}$ & $14 \pm 0.7$ & $13 \pm 0.6$ & $17 \pm 03$ \\
\hline Charge (mV) & 16 & 42 & 50 \\
\hline MMAD $(\mu \mathrm{m}) \pm$ GSD at an aerosol conc. of $0.5 \mathrm{mg} / \mathrm{m}^{3}$ & $1.9 \pm 2.9$ & $1.4 \pm 2.3$ & $1.3 \pm 2.2$ \\
\hline MMAD $(\mu \mathrm{m}) \pm$ GSD at an aerosol conc. of $2 \mathrm{mg} / \mathrm{m}^{3}$ & - & - & $1.2 \pm 2.4$ \\
\hline MMAD $(\mu \mathrm{m}) \pm$ GSD at an aerosol conc. of $5 \mathrm{mg} / \mathrm{m}^{3}$ & - & $1.2 \pm 2.1$ & $1.1 \pm 2.3$ \\
\hline MMAD $(\mu \mathrm{m}) \pm \mathrm{GSD}$ at an aerosol conc. of $25 \mathrm{mg} / \mathrm{m}^{3}$ & $2.2 \pm 2.4$ & $1.0 \pm 2.5$ & - \\
\hline
\end{tabular}

a The surface reactivity was measured with the ferric reduction ability of serum (FRAS) assay (Gandon et al., 2017). The read-out of the FRAS assay is calibrated by a concentration series of Trolox (6-hydroxy-2,5,7,8tetramethylchroman-2-carboxylic acid, a Vitamin E analogon) and the result expressed as nmol Trolox Equivalent Unites (TEU) $/ \mathrm{m}^{2} \mathrm{NP}$.

$\mathrm{BET}=$ Brunauer, Emmett and Teller method to measure surface area, MMAD=mass median aerodynamic diameter, GSD=geometric standard deviation, -: no data available 


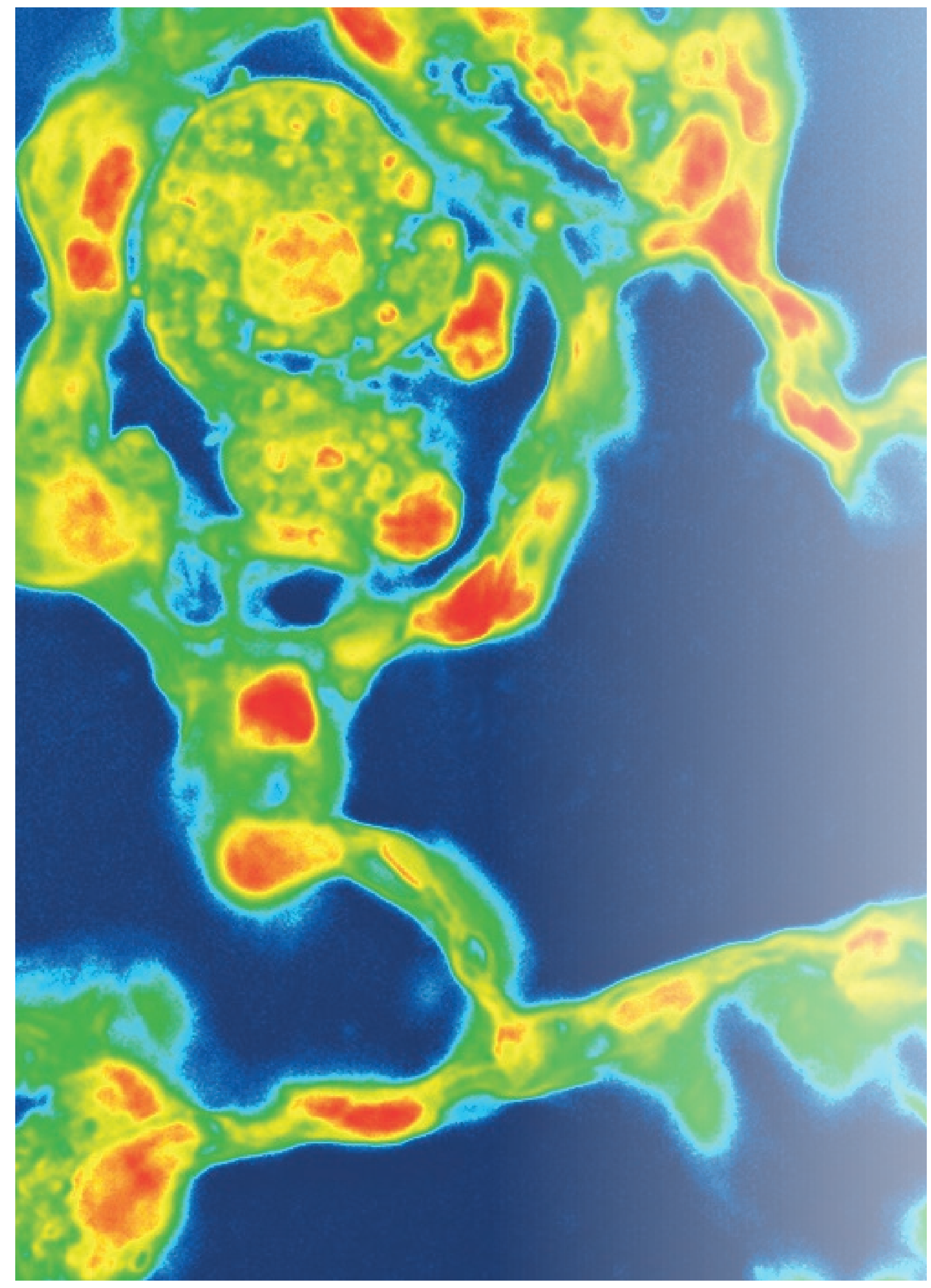




\section{Chapter 4}

\section{Multi-omics approaches confirm metal ions mediate the main toxicological pathways of metal- bearing nanoparticles in lung epithelial A549 cells}

Susan Dekkers*1, Tim D. Williams², Jinkang Zhang ${ }^{2}$, Jiarui (Albert) Zhou ${ }^{3}$, Rob J. Vandebriel', Liset J.J. De La Fonteyne', Eric R. Gremmer', Shan He³, Emily J. Guggenheim ${ }^{4}$, Iseult Lynch ${ }^{4}$, Flemming R. Cassee 1,5, Wim H. De Jong' ${ }^{\text {, Mark R. Viant }}{ }^{2}$

Environmental Science: Nano 5:1506-1517 (2018)

Reproduced by permission of The Royal Society of Chemistry

${ }^{1}$ National Institute for Public Health and the Environment (RIVM), Bilthoven, The Netherlands,

2 School of Biosciences, The University of Birmingham, Birmingham, B15 2TT, UK,

${ }^{3}$ Centre for Systems Biology, The University of Birmingham, Birmingham, B15 2TT, UK,

${ }^{4}$ School of Geography Earth and Environmental Sciences, The University of Birmingham, Birmingham, B15 2TT, UK,

${ }^{5}$ Institute of Risk Assessment Sciences, Utrecht University, Utrecht, The Netherlands,

* These authors contributed equally 


\begin{abstract}
The toxicity of silver ( $\mathrm{Ag}$ ) and zinc oxide ( $\mathrm{ZnO})$ nanoparticles (NPs) has been associated with their dissolution or ability to release metal ions while the toxicity of cerium dioxide $\left(\mathrm{CeO}_{2}\right)$ NPs has been related to their ability to induce or reduce oxidative stress dependent on their surface redox state. To examine the underlying biochemical mechanisms, multiple omics technologies were applied to characterise the responses at the molecular level in cells exposed to various metal-based particles and their corresponding metal ions. Human lung epithelial carcinoma cells (A549) were exposed to various $\mathrm{Ag}$, $\mathrm{ZnO}$, and $\mathrm{CeO}_{2} \mathrm{NPs}, \mathrm{Ag}$ and $\mathrm{ZnO}$ micro-sized particles (MPs), $\mathrm{Ag}$ ions $\left(\mathrm{Ag}^{+}\right)$and zinc ions $\left(\mathrm{Zn}^{2+}\right)$ over a $24 \mathrm{~h}$ time course. Molecular responses at exposure levels that caused $\sim 20 \%$ cytotoxicity were characterised by direct infusion mass spectrometry lipidomics and polar metabolomics and by RNAseq transcriptomics. All Ag, $\mathrm{Zn}$ and $\mathrm{ZnO}$ exposures resulted in significant metabolic and transcriptional responses and the great majority of these molecular changes were common to both ionic and NP exposures and characteristic of metal ion exposure. The low toxicity $\mathrm{CeO}_{2}$ NPs elicited few molecular changes, showing slight evidence of oxidative stress for only one of the four $\mathrm{CeO}_{2}$ NPs tested. The multiple omics analyses highlight the main pathways implicated in metal ions-mediated effects. These results can be used to establish adverse outcome pathways as well as strategies to group nanomaterials for risk assessment.
\end{abstract}




\section{Introduction}

Although nanomaterials are increasingly used in many different applications, detailed knowledge on the underlying biochemical mechanisms by which they may induce harmful effects on humans and the environment is lacking. Several possible mechanisms of action have been proposed ${ }^{1}$. One of the proposed mechanisms of action is related to the release of metal ions. For both silver ( $\mathrm{Ag}$ ) and zinc oxide ( $\mathrm{ZnO}$ ) nanoparticles (NPs), toxicity is often related to their dissolution or ability to release metal ions ${ }^{2,3}$. However, studies comparing the toxicity of these NPs with that of their ionic forms indicate that the toxicity of the NPs cannot always be fully explained by the release of metal ions ${ }^{4,5}$. Another proposed mechanism of action is via the induction of oxidative stress through the generation of reactive oxygen species (ROS). Cerium dioxide $\left(\mathrm{CeO}_{2}\right)$ NPs may have oxidative as well as anti-oxidative properties, depending on their redox surface state. The ability to shift valence states from $\mathrm{Ce}^{3+}$ to $\mathrm{Ce}^{4+}$ or from $\mathrm{Ce}^{4+}$ to $\mathrm{Ce}^{3+}$ at the surface of the NPs is suggested to influence the ability of the $\mathrm{CeO}_{2}$ NPs to either scavenge or generate reactive oxygen species (ROS), respectively, subsequently increasing or decreasing the ability to induce oxidative stress ${ }^{6,7}$. However, previous studies comparing $\mathrm{CeO}_{2} \mathrm{NPs}$ with different valence states indicate that the mechanisms by which the redox surface status of NPs influences the toxicity are not yet fully understood ${ }^{7}$.

Transcriptomics (gene transcriptional profiling), metabolomics (profiling of polar metabolites), proteomics (profiling of proteins) and lipidomics (profiling of lipids) are valuable non-hypothesis driven methods to gain insight into the mechanisms of actions or pathways leading to biological effects of NPs on living organisms, especially when these approaches are combined into a multi-omics approach to explore a larger molecular landscape ${ }^{8}$. Computational modelling can be used to search for molecular signatures that can contribute to the discovery of molecular key (initiating) events within adverse outcome pathways (AOPs), i.e. mechanistically based molecular changes that are related to both an (upstream) molecular initiating event and (downstream) key events leading to higher levels of phenotypic change ${ }^{9}$. These signatures can be used in the screening, ranking and risk assessment of nanomaterials. When designing a multi-omic study, it is important to generate time-resolved data to be able to follow the different molecular responses within a pathway leading to a biological response ${ }^{10}$. Multi-omics approaches have not yet been widely used within the field of nanotoxicology ${ }^{11,12}$.

In this study, toxicological, analytical and computational methods are combined to 1) identify the molecular mechanisms by which $\mathrm{Ag}, \mathrm{ZnO}$ and $\mathrm{CeO}_{2} \mathrm{NPs}$ induce toxicity and 2) investigate the influence of dissolution and redox surface state on the NP toxicity using transcriptomics, metabolomics and lipidomics. A549 lung epithelial cells were exposed to nano, micro and ionic forms of $\mathrm{Ag}, \mathrm{Zn}$ or $\mathrm{ZnO}$ and various $\mathrm{CeO}_{2} \mathrm{NPs}$ over a 24 hour time course. A549 cells 
were selected as they are lung epithelial cells and inhalation is considered an important route of exposure in occupational settings and for consumers using spray products ${ }^{13,14}$. To investigate the role of dissolution and ionic release on the pathways leading to adverse effects of metal (oxide) NPs, Ag and ZnO NPs and MPs as well as $\mathrm{Ag}^{+}$and $\mathrm{Zn}^{2+}$ were studied in parallel. In addition, $\mathrm{CeO}_{2} \mathrm{NPs}$ with different amounts of zirconium ( $\mathrm{Zr}$ )-doping were studied as a means to investigate the effect of the redox surface state on the biological response. $\mathrm{Zr}$-doping increases the $\mathrm{Ce}^{3+}: \mathrm{Ce}^{4+}$-ratio and is therefore expected to increase the antioxidant potential of the $\mathrm{CeO}_{2} \mathrm{NPs}^{6,7}$. Concentrations of NPs and their equivalent ions that induced approximately $20 \%$ cytotoxicity after $24 \mathrm{~h}$ exposure were chosen for this study, aiming to achieve a similar level of cellular damage in all cases. Importantly, temporal responses were investigated by sampling after different exposure times (1, 6 and $24 \mathrm{~h})$ to characterize the development of the toxicological responses over time.

\section{Materials and Methods}

\section{Nanomaterials: selection, dispersion and characterisation}

$\mathrm{Ag}, \mathrm{ZnO}$ and $\mathrm{CeO}_{2}$ NPs were selected because of their expected modes of action that involve either the release of ions or their ability to generate or scavenge ROS. An overview of the physicochemical characteristics of the selected materials is given in Table 1. When provided as powder the micro- and nano-sized particles were dispersed using the previously published protocol by Jensen et al. ${ }^{15}$. For a final stock concentration of $2.56 \mathrm{mg} / \mathrm{mL}$ the powder was pre-wetted with 0.5 vol\% ethanol and dispersed in water with $0.05 \% \mathrm{w} / \mathrm{v}$ bovine serum albumin from Sigma-Aldrich Chemie (Zwijndrecht, The Netherlands), and sonicated for 16 minutes on ice using a 400 Watt Branson Sonifier S-450D set at $10 \%$ amplitude with a $3 \mathrm{~mm}$ probe (Branson Ultrasonics Corp., Danbury, CT, USA). When provided as dispersions, the NPs were vortexed for $15 \mathrm{~s}$ and sonicated for 5 mins in an ultrasonic bath (Branson CPX2800, 40 $\mathrm{kHz}, 110 \mathrm{~W}$ ) to re-disperse any possible agglomerates.

\section{Cell culture and exposures}

A549 cells were obtained from ATCC (VA, USA). The cells were cultured in tissue culture flasks in RPMI 1640 medium with Glutamax (Gibco, ThermoFisher Scientific Inc., Landsmeer, the Netherlands) supplemented with 10\% Fetal Bovine Serum (FBS, Greiner BioOne BV, Alphen aan de Rijn, the Netherlands) and $1 \%$ penicillin/streptomycin (Gibco). Cells were cultured at $37^{\circ} \mathrm{C}$ in a humidified atmosphere of $5 \% \mathrm{CO}_{2}$ in air. The adherent cells were harvested by a short incubation with 0.5\% EDTA trypsin in $\mathrm{Ca} / \mathrm{Mg}$ free Dulbecco's Phosphate Buffered Saline (Gibco).

To determine the $\mathrm{EC}_{20}$ (effective concentration resulting in $20 \%$ cytotoxicity) dose-response studies were performed. A549 cells were harvested and counted $24 \mathrm{~h}$ before exposure. Twenty thousand $\left(2 \times 10^{4}\right)$ cells were seeded in wells of 96-well plates in $100 \mu \mathrm{L}$ supplemented 
RPMI 1640 medium. After $24 \mathrm{~h}$ incubation a semi-confluent monolayer of cells was obtained and the cells were exposed to the various materials. Cell survival (i.e. cytotoxicity) was determined after $24 \mathrm{~h}$ of exposure by a colorimetric assay using cell proliferation reagent WST-1 (Roche, Sigma-Aldrich Chemie). All exposures were performed in triplicate. Doseresponse modelling and derivations of the $\mathrm{EC}_{20}$ were performed using PROAST software ${ }^{16}$ version 60.1. For the four $\mathrm{CeO}_{2} \mathrm{NPs}$ and $\mathrm{Ag} \mathrm{MP} \mathrm{no} \mathrm{EC}_{20}$ was obtained, since the highest concentration tested $(128 \mu \mathrm{g} / \mathrm{mL})$ resulted in less than $20 \%$ cytotoxicity. An overview of the $\mathrm{EC}_{20}$ values and confidence intervals can be found in Electronic Supplementary Information (ESI) 1. Dissolution of nanoparticles and microparticles in cell culture medium was measured using ICP-OES (ESI 2). 


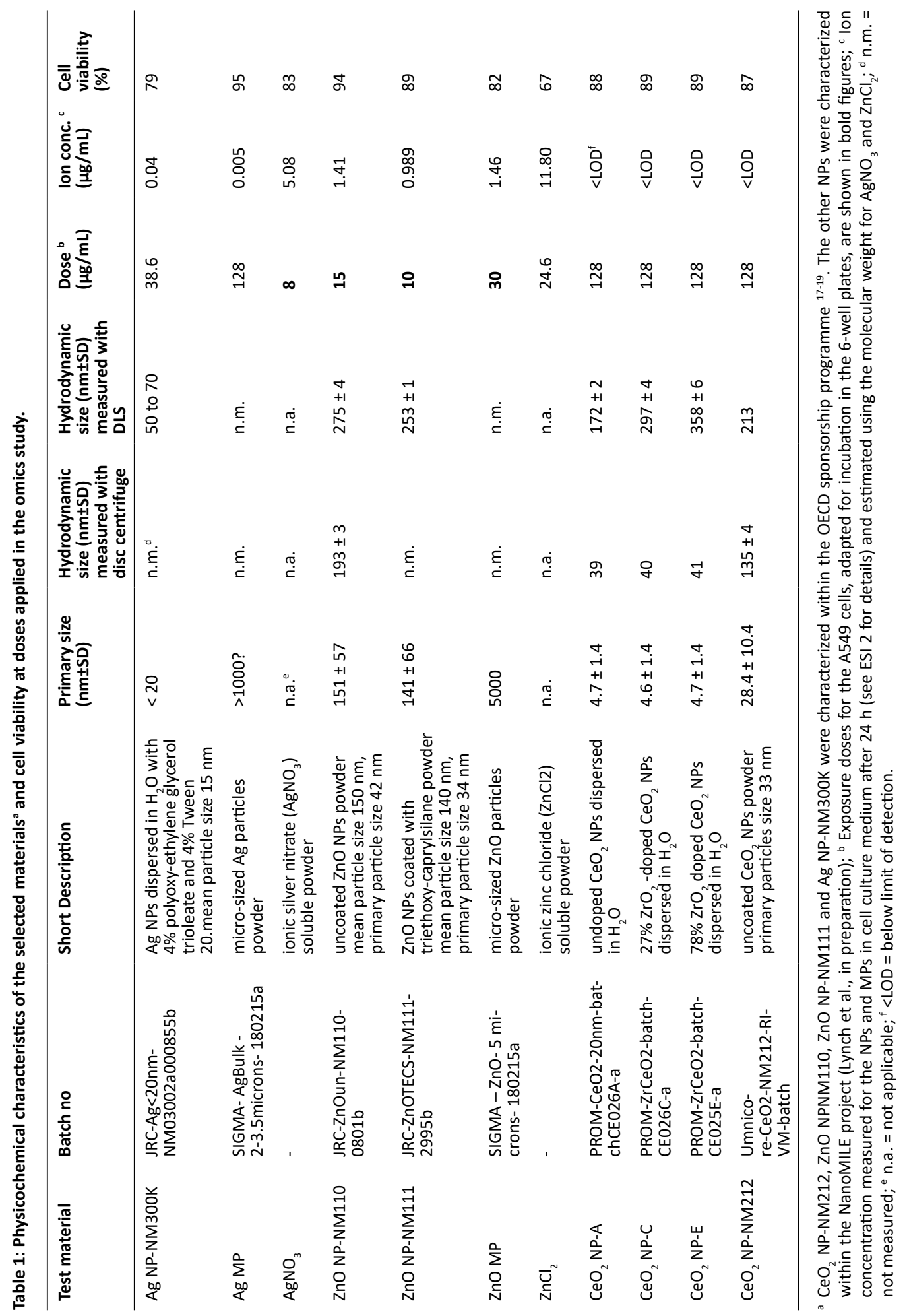


For the omics studies, $8 \times 10^{5}$ cells per well were seeded in 6-well plates and cultured for $18 \mathrm{~h}$, after which the cells were exposed to the determined $\mathrm{EC}_{20}$ concentrations or to 128 $\mu \mathrm{g} / \mathrm{mL}$ for the particles where $\mathrm{EC}_{20}$ was not reached. Because the cytotoxicity of the A549 cells was higher in the 6-well plates compared to the 96-well plates, several concentrations were adapted and applied as presented in Table 1. Omics analyses were carried out on independent replicates from 14 exposure and control groups. Biological replication was, for polar metabolomics $n=6$, lipidomics $n=6$ and transcriptomics $n=4$. Different cell plates were exposed for $t=1, t=6$ and $t=24 h$ to monitor changes in the molecular responses over time. At $t=24 \mathrm{~h}$, additional control wells were included to measure the actual cytotoxicity and possible interference of the materials with the viability assay. After exposure, the cells (approximately $2 \times 10^{6}$ per well) were quickly washed with PBS (phosphate buffered saline) twice at room temperature after which the 6-well plates were deep frozen by quenching on liquid nitrogen $\left(-196^{\circ} \mathrm{C}\right)$ and stored at $-80^{\circ} \mathrm{C}$ until extraction for omics evaluation.

\section{Omics Analyses}

Brief descriptions of the methods used for omics analysis are shown. Full methodological details are provided in ESI 3.

\section{Extraction of metabolites and lipids}

Cells were harvested then vortexed in methanol:chloroform:water ( $\mathrm{v} / \mathrm{v} / \mathrm{v}$ at 1:1:0.9) and the phases separated by centrifugation. The polar phase was dried in a speed vac concentrator (Thermo Savant, Holbrook, NY) for $4 \mathrm{~h}$. The non-polar phase was dried under a stream of nitrogen for $5 \mathrm{~min}$. All dried samples were then frozen at $-80^{\circ} \mathrm{C}$ until analysis.

\section{Direct infusion mass spectrometry (DIMS)}

The DIMS analysis method used was similar to previous studies ${ }^{20,21}$. Dried extracts were re-suspended in 80:20 (v/v) methanol:water with $0.25 \%$ formic acid (for positive ion mode analysis of polar extracts) or $80 \mu \mathrm{L}$ 2:1 methanol:chloroform with $5 \mathrm{mM}$ ammonium acetate (for negative ion mode analysis of lipids). Samples were analysed (in quadruplicate) using direct infusion mass spectrometry ( $Q$ Exactive, Thermo Fisher Scientific, Germany) in positive ion mode (for polar metabolomics) or negative ion mode (for lipidomics), utilising a Triversa nanoelectrospray ion source (Advion Biosciences, Ithaca, NY, USA).

\section{Metabolomics Data Processing}

Mass spectra were recorded using the selected ion monitoring (SIM) stitching approach from $\mathrm{m} / \mathrm{z}$ 50-620 (for polar metabolomics) or from m/z 50-1020 (for lipidomics) and then processed using custom-written Matlab scripts as previously reported ${ }^{22,23}$. The resulting matrices of peak intensities (termed "DIMS dataset") were probabilistic-quotient normalised (PQN) and intensity-drift corrected using a Quality Control-Robust Spline Correction (QC- 
$\mathrm{RSC}$ ) algorithm. Finally, the missing values were imputed using the k-nearest neighbours (KNN) algorithm. For multivariate analysis, generalized log (Glog) transformation of the DIMS dataset was performed.

\section{RNA seq gene expression profiling}

\section{Sequencing}

Total RNA was extracted from A549 cells using a micro RNeasy Kit (Qiagen, Crawley, UK). All RNA libraries were produced using the Biomek FxP (Beckman Coulter A31842) with Ultra Directional RNA Library Prep Kit (New England Biolabs E7420L) and NEBnext Multiplex Oligos for Illumina Dual Index Primers (New England Biolabs E7600S), using provided protocols and 500ng of total RNA. Multiplex library clustering and sequencing was performed upon the HiSeq2500 (Illumina) with HiSeq Rapid Cluster Kit v2 (Illumina GD-402-4002) at 12pM library concentration with 10\% PhiX Control v3 spiked in (Illumina FC-110-3001). The sequencing run was carried out using HiSeq Rapid SBS Kit v2 (Illumina FC-402-4021).

\section{RNA seq Data Processing}

The binary base call (BCL) files were converted to FASTQ format (containing a biological sequence and its corresponding quality scores) using Illumina bcl2fastq conversion software (v1.8.4). Sequences were then trimmed using Trimmomatic (v0.36). Five low quality samples were identified and removed accordingly. The FASTQ files were aligned to the GENCODE human transcript sequences (release 25, GRCh38.p7) using Bowtie2 (v2.3.0). The resulting Sequence Alignment Map (SAM) data were converted into Browser Extensible Data (BED) format using SAMtools (v1.3.1) and bamToBed (v2.19.1). Finally, the RNA read counts were extracted from the BED files with a Python script. To provide gene-level analysis, the RNA reads were collapsed to the counts of their coding genes. The gene annotation information was retrieved from the Ensembl database (release 87).

\section{Omics Data Analysis}

Putative metabolite annotations were added using MI-Pack ${ }^{24}$. ANOVA, t-tests and principal components analyses (PCA) were performed in Genespring (v7.3.1. Agilent) using multiple testing corrections ${ }^{25}$. DESeq $2^{26}$ was used for differential gene expression analysis with a $\mathrm{q}<0.05$ cut-off. Combined gene and metabolite pathway over-representation analyses were performed with IMPaLA ${ }^{27}$, using gene identifiers and Human Metabolite Database (HMDB) identifiers ${ }^{28}$ for each peak identified as significantly altered as input lists. Comparative pathway analyses were performed with Ingenuity Pathway Analysis (IPA; Qiagen) on combined sets of genes, lipids and polar metabolite identifiers. Raw transcriptomic data and experimental details are archived at ArrayExpress (accession number: E-MTAB-5734). 


\section{Results}

In total, 259 polar metabolomic, 250 lipidomic and 156 transcriptomic samples passed the quality control metrics. Four RNAseq samples were removed prior to further analysis due to anomalously low counts. The full results of univariate analyses comparing each exposure group with its time-matched control group are shown in ESI 4. Comparisons were made with time-matched controls since gene expression and metabolite profiles varied significantly with time between the control groups sampled at $1 \mathrm{~h}, 6 \mathrm{~h}$ and $24 \mathrm{~h}$. Figure 1 illustrates the overall numbers of significantly changing $(q<0.05)$ genes and metabolites in comparison with time-matched controls. The numbers of molecular (transcript and metabolic) changes at $24 \mathrm{~h}$ correlated significantly with cytotoxicity (Table 1) for all silver (Ag MP, Ag NP and $\left.\mathrm{Ag}^{+}\right)$exposures $\left(r^{2}=0.98 ; p<0.01\right)$ and all zinc $\left(\mathrm{ZnO} M P, \mathrm{ZnO} N P\right.$ and $\left.\mathrm{Zn}^{2+}\right)$ exposures $\left(\mathrm{r}^{2}=0.85\right.$; $\mathrm{p}<0.03$ ) but not for the $\mathrm{CeO}_{2} \mathrm{NP}$ exposures. All silver exposures resulted in significantly more changes than $\mathrm{CeO}_{2}$ exposures, and all zinc exposures led to the most numerous alterations. Silver exposures ranked from the largest to the smallest effect in the order $\mathrm{Ag}^{+}$, $\mathrm{Ag} \mathrm{NP}$ and then Ag MP, whereas zinc exposures ranked highest for $\mathrm{Zn}^{2+}$, followed by $\mathrm{ZnO}$ MP, ZnO NP-NM110 and ZnO NP-NM111. These rank orders matched the orders of ionic concentrations determined by dissolution analysis (Table 1). $\mathrm{CeO}_{2} \mathrm{NP}$ exposures resulted in few gene expression or metabolic changes, and ionic dissolution was below the limit of detection. Of these few changes, $\mathrm{CeO}_{2} \mathrm{NP}-\mathrm{A}$ (undoped $\mathrm{CeO}_{2} \mathrm{NPs}$ ) was the only $\mathrm{CeO}_{2} \mathrm{NP}$ that increased metabolites putatively identified as cysteine at $1 \mathrm{~h}(3.5$ fold; $\mathrm{q}<0.032)$ and y-glutamylcysteine at $6 \mathrm{~h}$ (1.5 fold; $\mathrm{q}<0.02)$.

PCA scores plots of transcriptomic and polar metabolomic data after $6 \mathrm{~h}$ of exposure are shown in Figure 2, with PCAs for all other timepoints, as well as for the lipidomics data, shown in ESI 5. For silver there was grouping of replicate samples and separation from the controls was apparent for all exposure groups. For zinc, replicate samples grouped tightly and clearly diverged from the control group along PC1, with the degree of divergence corresponding with the number of molecular changes outlined above (see also Figure 1). There was little or no apparent grouping of the $\mathrm{CeO}_{2}$ NP samples or divergence from the control group. 


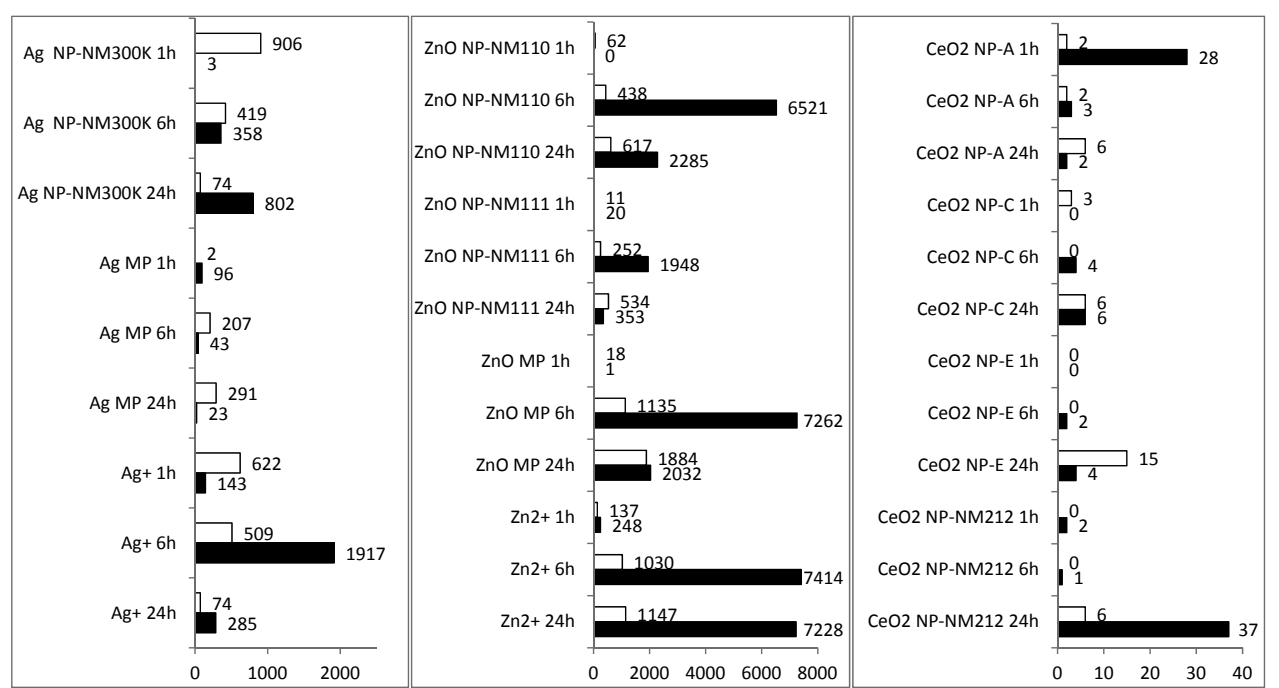

Figure 1: Number of genes significantly differentially expressed (black bars) and metabolite peaks significantly altered in concentration (clear bars) $(\mathrm{q}<0.05)$ in $\mathrm{A} 549$ cells after silver, zinc, or $\mathrm{CeO}_{2} \mathrm{NPs}, \mathrm{MPs}$ and/or ionic exposures for 1,6 or $24 \mathrm{~h}$.

Silver $6 \mathrm{~h}$
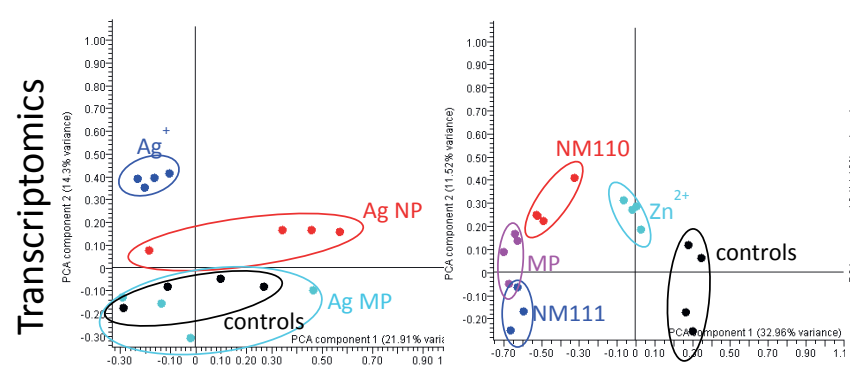

$\mathrm{CeO}_{2} 6 \mathrm{~h}$

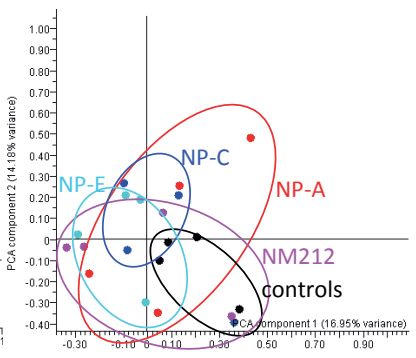

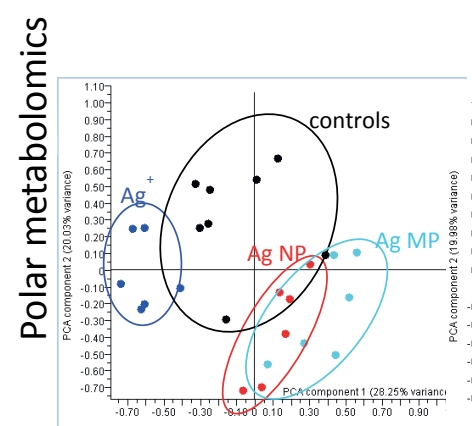
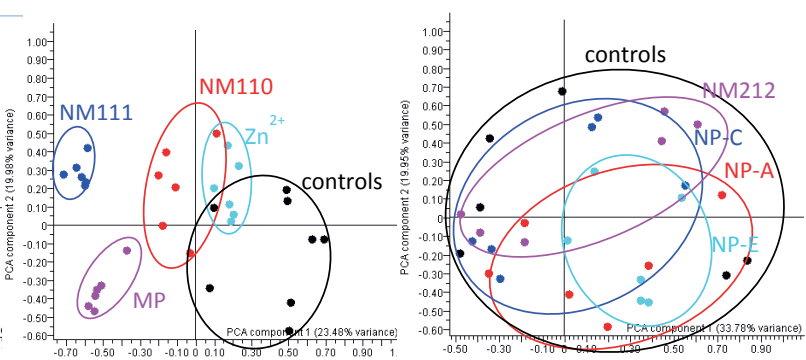

Figure 2: Principal components analysis scores plots of transcriptomics and metabolomics data from A549 cells exposed to silver, zinc, or $\mathrm{CeO}_{2}$ for $6 \mathrm{~h}$. For silver, control samples are shown in black, Ag NP treated in red, Ag MP in cyan, $\mathrm{Ag}^{+}$in blue. For zinc, control samples are shown in black, ZnO NP-NM110 in red, ZnO NP-NM111 in blue, Zn ${ }^{2+}$ in cyan and $\mathrm{ZnO} M P$ in pink. For $\mathrm{CeO}_{2}$, control samples are shown in black, $\mathrm{CeO}_{2} \mathrm{NP}-\mathrm{A}$ treated in red, $\mathrm{CeO}_{2} \mathrm{NP}-\mathrm{C}$ in blue, $\mathrm{CeO}_{2}$ NP-E in cyan and $\mathrm{CeO}_{2}$ NP-NM212 in pink. PCAs for all timepoints are shown in ESI 5. 
IMPaLA pathway over-representation analysis results are shown in full in ESI 6, while overrepresentation of selected pathways is illustrated in Figure 3. All silver and zinc exposures at $6 \mathrm{~h}$ and $24 \mathrm{~h}$ resulted in significant enrichment of the terms 'Response to metal ions' and 'Metallothioneins bind metals'. Terms relating to the heat shock response were enriched in the same groups at $6 \mathrm{~h}$ but not at $24 \mathrm{~h}$, except for the $\mathrm{Ag}^{+}$and $\mathrm{Zn}^{2+}$ exposures where they persisted. Other enriched terms, including 'Translation', 'Nonsense-Mediated Decay', 'Apoptosis' and 'Immune System' were highlighted with all zinc exposures and either $\mathrm{Ag}^{+}$ or Ag NP exposure at $6 \mathrm{~h}$ and sometimes also at $24 \mathrm{~h}$. $\mathrm{Zn}^{2+}, \mathrm{ZnO} \mathrm{MP}$ and $\mathrm{ZnO} \mathrm{NP}-\mathrm{NM} 110$ repressed molecules related to $\mathrm{DNA}$ repair. $\mathrm{CeO}_{2}$ exposures elicited few molecular changes and showed no enriched pathway annotations, apart from 'HIF-1 alpha transcription network' induced at $24 \mathrm{~h}$ with $\mathrm{CeO}_{2} \mathrm{NM} 212$ and 'ID signalling pathway' repressed at 6 and 24h with $\mathrm{CeO}_{2}$ NP-A,- $\mathrm{C}$ and $\mathrm{E}$.

Ingenuity Pathway Analysis (IPA) Comparison Analyses were used to compare molecular pathway responses to the various silver and zinc exposures. Figure 4 shows the top 20 canonical pathways and the top 20 'diseases and bio-functions' associated with the silver and zinc exposures, ordered by function or process from IPA's Pathway Activity Analysis function, representing predicted pathway activation or inhibition. Particularly prominent for silver was the Nrf2-mediated oxidative stress response pathway, predicted to be activated by $\mathrm{Ag} \mathrm{NP}$ at $6 \mathrm{~h}$ and by $\mathrm{Ag}^{+}$at all timepoints. The $\mathrm{Nrf2}$ pathway was also predicted to be activated by all $\mathrm{Zn}$ exposures at $6 \mathrm{~h}$, but repressed at $24 \mathrm{~h}$. The canonical pathway comparison of silver exposures was otherwise dominated by modulation of several molecular signalling pathways, particularly with $\mathrm{Ag}^{+}$at $6 \mathrm{~h}$. All $\mathrm{Zn}$ exposures resulted in very similar profiles of predicted pathway activation, highlighting co-ordinated induction of signalling pathways at $6 \mathrm{~h}$, followed by repression at $24 \mathrm{~h}$, except for $\mathrm{Zn}^{2+}$ for which these pathways were predicted to still be activated at $24 \mathrm{~h}$. Data from $\mathrm{CeO}_{2} \mathrm{NP}$ exposures were not used due to the low numbers of responsive molecules.

To identify candidate nano-specific responses, t-tests were performed comparing, for silver, the Ag NP group versus respectively the control, $\mathrm{Ag}^{+}$and $\mathrm{Ag} \mathrm{MP}$ groups. Molecules were only selected if statistically significantly $(F D R<0.05)$ changed in all comparisons. A similar procedure was followed for ZnO NP-NM110 and ZnO NP-NM111. The results of these comparisons and IMPaLA pathway analyses using these data are shown in Figure $\mathbf{5}$ and ESI 7. For Ag NPs, $17.6 \%$ of transcriptional and $22 \%$ of metabolic changes were assessed as candidate nano-specific. The induced molecules associated with several pathways, particularly those concerned with amino acid metabolism, while the decreasing molecules associated with glycolysis and galactose metabolism and reduced transcription relating to phase II xenobiotic metabolism. ZnO NP-NM110 elicited only 12 (0.15\%) candidate nanospecific changes and ZnO NP-NM111 elicited 22 (0.77\%), mostly reduction in metabolites associated with galactose metabolism. 


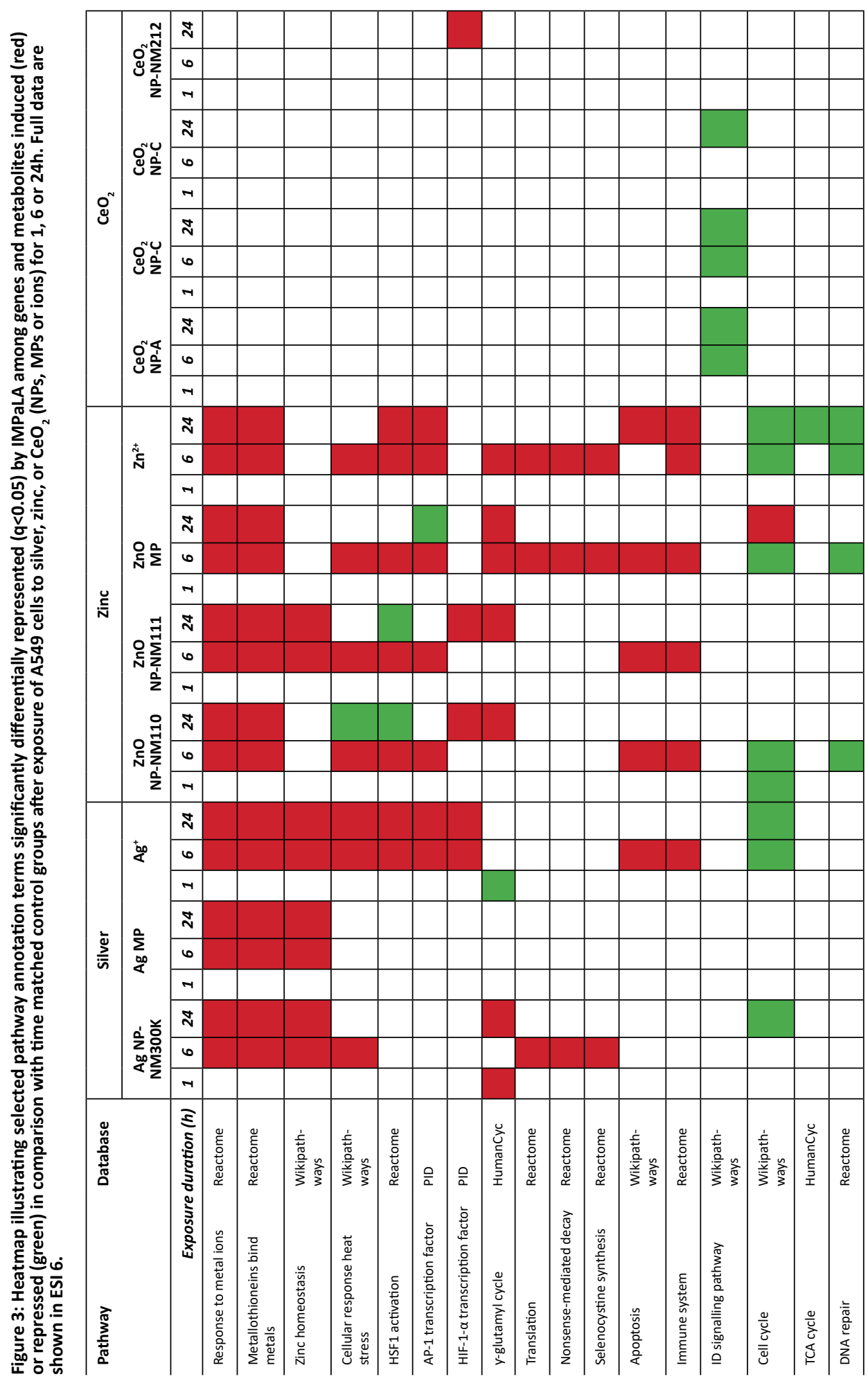




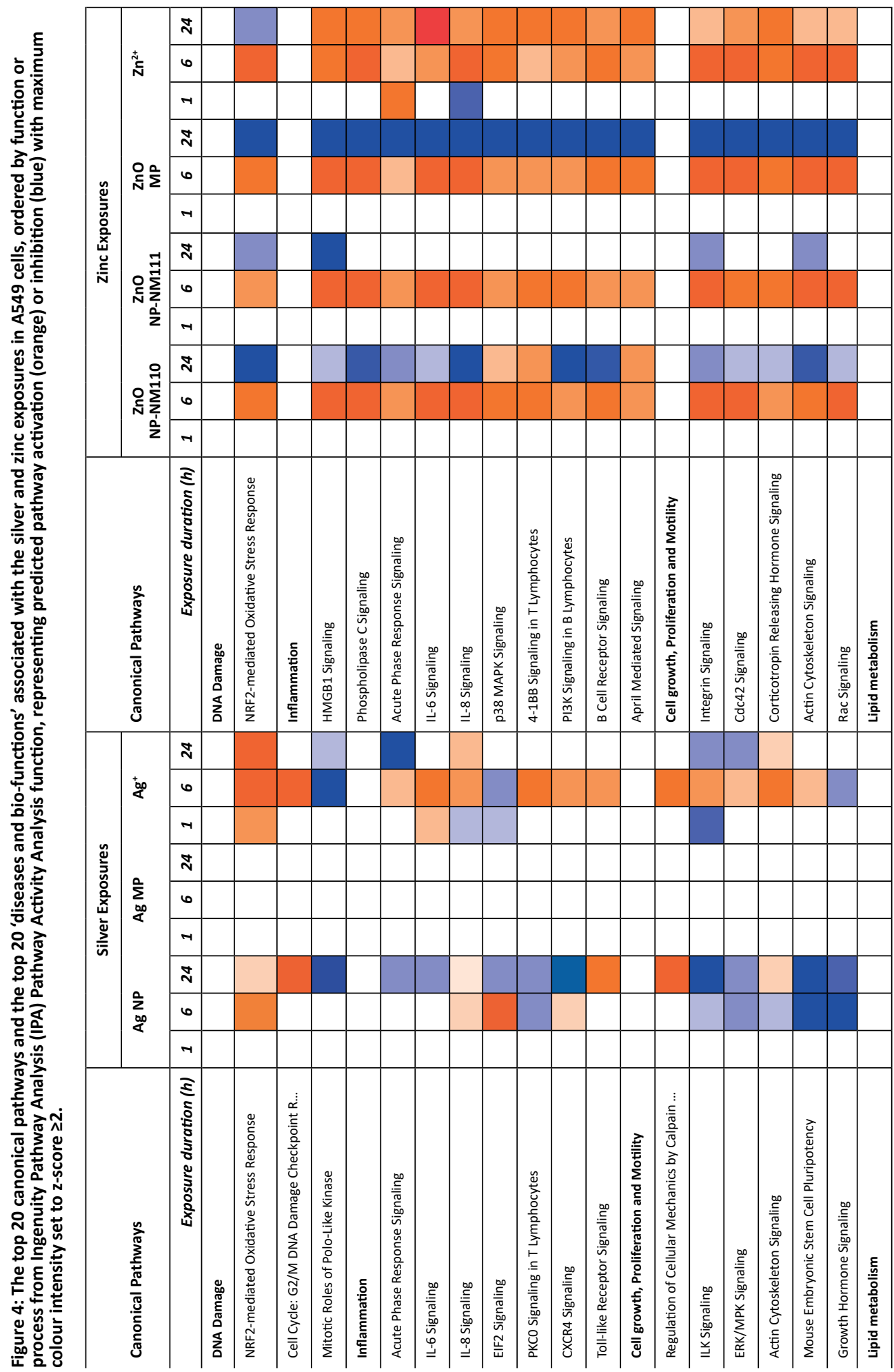




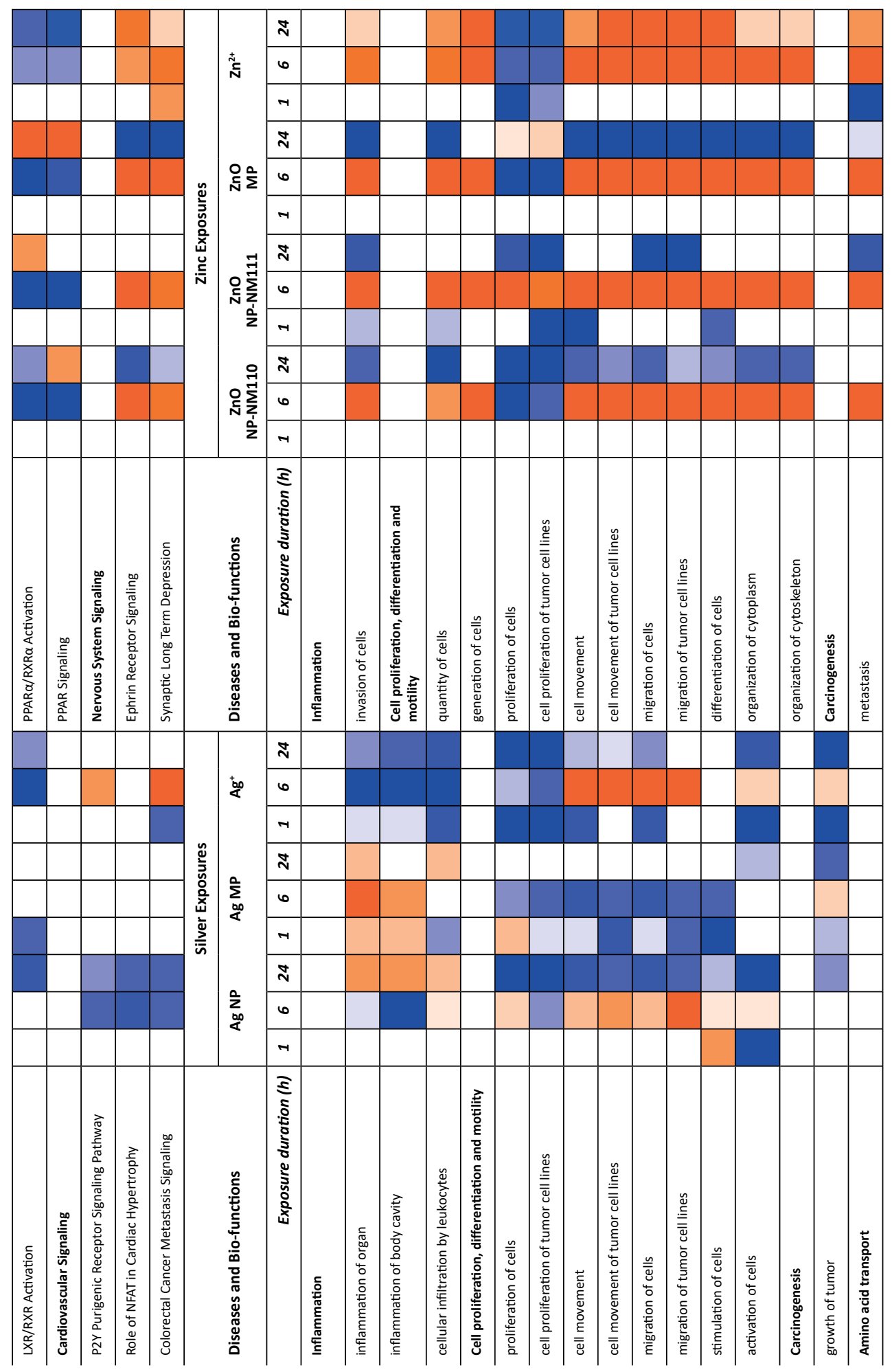




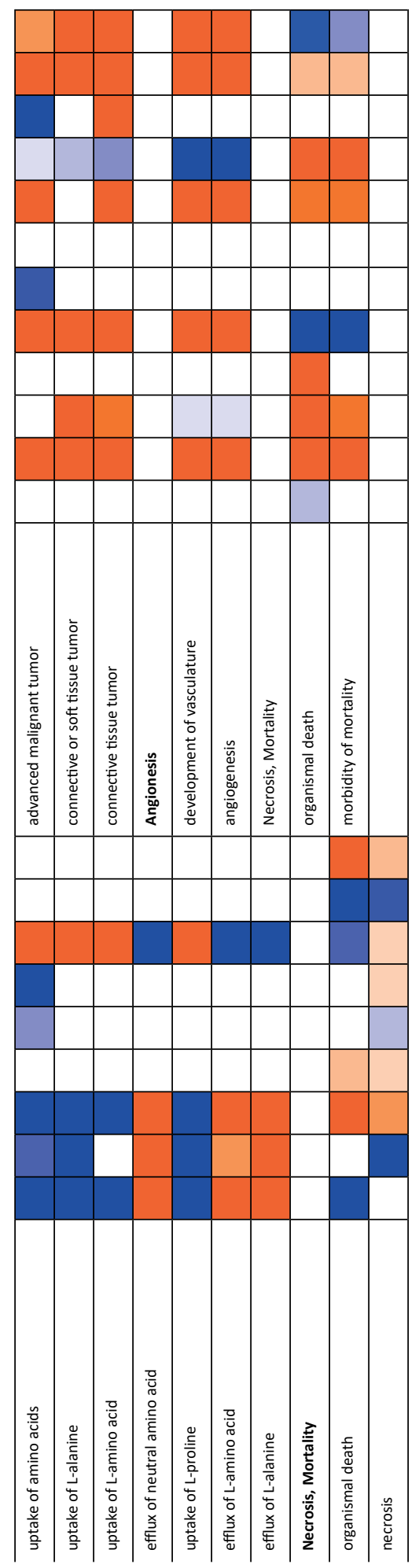


Figure 5: Heatmap illustrating selected pathway annotation terms significantly differentially represented $(q<0.05)$ by IMPaLA among candidate nano-specific transcripts and metabolites induced (red) or repressed (green) in comparison with control, microparticle and ionic exposures over all timepoints. Full data are shown in ESI 7.

\begin{tabular}{|c|c|c|c|c|}
\hline Pathway & Database & Ag NP & $\begin{array}{l}\text { ZnO NP- } \\
\text { NM110 }\end{array}$ & $\begin{array}{l}\text { ZnO NP- } \\
\text { NM111 }\end{array}$ \\
\hline Amino Acid interconversion & Wikipathways & & & \\
\hline Amino Acid metabolism & Wikipathways & & & \\
\hline SLC-mediated transmembrane transport & Reactome & & & \\
\hline Phase II conjugation & Wikipathways & & & \\
\hline Amino acid transport across the plasma membrane & Reactome & & & \\
\hline y-glutamyl cycle & HumanCyc & & & \\
\hline Glucuronidation & Wikipathways & & & \\
\hline Xenobiotics metabolism & EHMN & & & \\
\hline Glycolysis & SMPDB & & & \\
\hline Bile secretion - Homo sapiens (human) & KEGG & & & \\
\hline Galactose Metabolism & SMPDB & & & \\
\hline Amino sugar and nucleotide sugar metabolism & KEGG & & & \\
\hline
\end{tabular}




\section{Discussion}

In our study molecular changes were sought that were unique to the nanomaterial exposures, not appearing in response to the ionic or micro-sized particle exposures at any timepoint. These were termed 'candidate nano-specific responses' as only three timepoints were examined for each exposure, raising the possibility of these responses having occurred at an unexamined timepoint in the non-NP exposures. For silver there was evidence for candidate nano-specific changes supported by both the transcriptomics and metabolomics data (Figure 5 and ESI 7). These were related to increases in amino-acid transport, reduced glycolysis and galactose metabolism and reduced glucuronidation and xenobiotic metabolism. Potentially these changes could result in lower capacity to detoxify organic xenobiotics and it could be instructive to compare modulation of organic xenobiotic toxicity in co-exposures with Ag NP or $\mathrm{Ag}^{+}$. However, since no dispersant control (for Ag NP-NM300K) was included in the omics study, some of these nano-specific changes may be caused by the dispersant (water with $4 \%$ polyoxyethylene glycerol trioleate and $4 \%$ Tween 20 ), instead of the Ag NPs. Although previous studies with the same Ag NPs and its dispersant indicated that the dispersant was not cytotoxic to A549 cells up to $256 \mu \mathrm{g} / \mathrm{mL},{ }^{29,} 30$ DNA damage was observed in the absence of cytotoxicity ${ }^{31}$ in A549 cells. For zinc there was very little evidence of candidate nano-specific responses, limited to metabolomics changes related in pathway analyses to a reduction in galactose metabolism. Although candidate nano-specific responses were found for Ag NPs, by far the majority (>78\%) of responses to the Ag NPs were also seen with $\mathrm{Ag}^{+}$ and $\mathrm{Ag} \mathrm{MP}$, as was found by NMR metabolomics in $\mathrm{HaCaT}$ cells $\mathrm{s}^{32}$, implying that nano-specific toxicity is likely a minor component compared with that elicited by silver ions.

All silver and zinc exposures induced transcription of genes responsive to metal ions at $6 \mathrm{~h}$ and $24 \mathrm{~h}$. Metallothionein induction was particularly notable, with MT1A, MT1B, MT1F, MT1G, MT1X, and MT2A highly and significantly induced with all $\mathrm{Ag}$ and $\mathrm{Zn}$ exposures but $M T 1 H$ and MT1E induced only with $\mathrm{Zn}$. Several of these $M T$ transcript inductions exceeded 1000 -fold, including MT1B with Ag NPs and $\mathrm{Ag}^{+}$at $6 \mathrm{~h}$ and $\mathrm{MT1H}$ with $\mathrm{ZnO} \mathrm{NM}-110, \mathrm{Zn}^{2+}$ and $\mathrm{Zn} \mathrm{MP}$ at $6 \mathrm{~h}$ and $24 \mathrm{~h}$. Additionally the zinc transporter SLC3OA1 (ZnT-1), responsible for export of zinc ions, was induced by both $\mathrm{Zn}$ and $\mathrm{Ag}$ exposures. $\mathrm{Ag}$ ions have been shown to release $\mathrm{Zn}$ ions in fibroblasts ${ }^{33}$.

Metallothioneins have long been considered biomarkers for metal ion exposure and oxidative stress $^{34}$ and metal-based NP studies frequently report their induction ${ }^{35,36}$. Metallothionein induction may be viewed as an adaptive response enabling cells to bind and sequester metal ions and for Ag MP exposure this response appeared effective, resulting in only 5\% cytotoxicity (Table 1) and few significant alterations in other biological pathways (Figure 3; ESI 6). However with the other Ag and $\mathrm{Zn}$ exposures resulting in higher cytotoxicity, it was apparent that this capacity was exceeded, leading to stress responses and cellular damage. 
The heat shock response was activated in all $6 \mathrm{~h}$ and $24 \mathrm{~h} \mathrm{Zn}$ exposures and with $\mathrm{Ag}^{2+}$ and Ag NPs; transcripts encoding the molecular chaperones HSPA1A, HSPA1L, HSPA6, HSPA7, HSPB1, HSPH1, HSP90AA1, HSP90AB1, DNAJB1 and DNAJB6 were induced, implying a response to protein damage.

Oxidative stress is a well-established outcome following NP exposure, including $\mathrm{Ag}, \mathrm{ZnO}$ and $\mathrm{CeO}_{2}, \mathrm{NPs}^{37,38}$. IPA highlighted the $\mathrm{Nrf2}$ mediated oxidative stress response as activated by $\mathrm{Ag}^{+}$and all $6 \mathrm{~h} \mathrm{Zn}$ exposures. Nrf-2 (NFE2L2) transcript was significantly but not highly induced ( $<1.6$-fold) with $\mathrm{Zn}^{2+}$ and $\mathrm{Zn} \mathrm{MP}$ and several key antioxidant enzyme transcripts were either mildly but significantly induced (SOD1, TXN, GLRX, GCLM, GSS) at $6 \mathrm{~h}$ or mildly repressed (CAT, GCLC, GSR, PRDX1). Aldehyde oxidase AOX1 was induced over 2-fold, as were heme oxygenase (HMOX1), several chaperones mentioned above and transcription factors FOS, JUN and ATF4. Transcription of KEAP1, a repressor of Nrf-2 signalling, was significantly repressed with $\mathrm{ZnO} \mathrm{NM}-110, \mathrm{Zn}^{2+}$ and $\mathrm{Zn} \mathrm{MP}$ at $6 \mathrm{~h}$. Nrf2 pathway induction has previously been found for $\mathrm{Ag} \mathrm{NPs}^{39}$ and for ionic $\mathrm{Ag}$ and $\mathrm{Zn}^{40}$. Several polar metabolite peaks that were putatively annotated as glutathione (GSH) followed a similar profile of a significant increase with $\mathrm{Ag}^{+}$at $1 \mathrm{~h}$ but a significant decrease with all zinc exposures at $6 \mathrm{~h}$ and $24 \mathrm{~h}$. GSH is the major intracellular antioxidant and its depletion implies vulnerability to further oxidative damage. GSH has previously been found to decrease with ZnO NP treatment in mouse livers and kidneys ${ }^{41,42}$.

A metabolite peak putatively annotated as cysteine was particularly highly increased with all Ag exposures, and amino acid concentrations were significantly altered with most exposures. This may represent an adaptive reorganisation of amino acid synthesis and transport. Expression of transcripts involved in translation, including those encoding ribosomal subunits, was increased with $\mathrm{Ag} \mathrm{NP}, \mathrm{ZnO} \mathrm{MP}$ and $\mathrm{Zn}^{2+}$ exposure. Interestingly nonsense mediated decay pathway transcripts were induced in the same exposures, implying an increase in mRNAs with premature stop codons. Potentially this could be due to an increased rate of DNA damage, as transcription of DNA repair genes was reduced, including those of the base excision repair, mismatch repair, nucleotide excision repair and double-strand break repair pathways, particularly with Zn ${ }^{2+}, \mathrm{Zn}$ MP and ZnO NP-NM110. DNA damage has previously been described for $\mathrm{Ag}^{+}, \mathrm{Ag} \mathrm{NPs}^{43,44}, \mathrm{Zn}^{2+}$ and $\mathrm{Zn} \mathrm{NPs}{ }^{45}$. Transcription of the stress-inducible AP-1 transcription factor genes was increased by $\mathrm{Zn}^{2+}$ and $\mathrm{ZnO} \mathrm{NP}$ exposure, and by $\mathrm{Ag}^{+}$, including induction of FOS, FOSB, FOSL1, JUN and JUNB. Cell cycle gene transcription was significantly repressed with both $\mathrm{Ag}$ and $\mathrm{Zn}$ exposures. These effects have previously been seen with $\mathrm{Ag} \mathrm{NPs}{ }^{43,46,47}$. There was an induction of transcription associated with apoptosis and with immune signalling with $\mathrm{Ag}^{+}$and all $\mathrm{Zn}$ exposures at $6 \mathrm{~h}$, persisting to $24 \mathrm{~h}$ with $\mathrm{Zn}^{2+}$. Both ionic and NP Ag and $\mathrm{Zn}$ can increase apoptosis in A549 cells ${ }^{48,49}$.

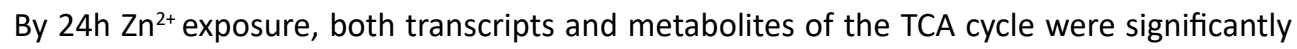


reduced, indicating major disruption of cellular respiration pathways, consistent with the bioenergetic disruption reported for ZnO NP exposure of A549 cells ${ }^{50}$.

These molecular alterations illustrate a progression from adaptive changes, such as metallothionein induction, to depletion of antioxidants, such as glutathione, repressed DNA repair and induction of apoptosis. Several of these molecular changes have been proposed as key events (KE) in the Adverse Outcome Pathway (AOP) paradigm ${ }^{51}$. Examples include increase in oxidative stress, activation of $\mathrm{Nrf}-2$, depletion of glutathione, repression of DNA repair and increased apoptosis. It is however apparent that many additional pathways, genes and metabolites were altered during the exposures to Ag and $\mathrm{Zn}$ (ESI 5 and 6) and that using a cell line model one can only examine those events leading up to cell death.

For $\mathrm{ZnO}$ NPs and MPs, responses indicative of damage tended to peak at $6 \mathrm{~h}$, with a reduction by $24 \mathrm{~h}$, for example acute phase signalling (Figure 5), while with $\mathrm{Zn}^{2+}$ these changes persisted to $24 \mathrm{~h}$, consistent with the greater cytotoxicity caused by this treatment. The responses to $\mathrm{Ag}$ and $\mathrm{Zn}$ clearly demonstrated the importance of measuring molecular responses over time in order to robustly assess molecular toxicity. At $1 \mathrm{~h}$ there were relatively few molecular pathway changes (Figure 3), at $6 \mathrm{~h}$ molecular responses indicative of toxicity had developed, but at $24 \mathrm{~h}$ for several exposures $\left(\mathrm{Ag}^{+}, \mathrm{Ag} \mathrm{MP}, \mathrm{ZnO}\right.$ NP-NM110, ZnO NP-NM111 and $\mathrm{Zn} \mathrm{MP)} \mathrm{the} \mathrm{responses} \mathrm{had} \mathrm{declined,} \mathrm{or} \mathrm{even} \mathrm{reversed} \mathrm{(Figure} \mathrm{4)} \mathrm{whereas} \mathrm{these} \mathrm{persisted}$ with the more cytotoxic $\mathrm{ZnCl}_{2}$ exposure. This time-dependence of molecular response can be explained by adaptive changes, such as induction of metallothioneins that ameliorate cellular damage by sequestering the metal ions, as illustrated by the Ag MP exposure. There may also be a time dependency in exposure to the metal ions, due to different uptake rates and dissolution kinetics of the different micro- and nano-sized particles resulting in different intracellular concentrations, or different intracellular compartmentalisation of the metal ions. In A549 cells and phagocytic murine macrophages, Ag NPs were associated with lysosomes ${ }^{52}$, whereas ionic $\mathrm{Ag}^{+}$was bound to metallothioneins ${ }^{53}$. ZnO NPs showed intracellular dissolution in lysosomes of macrophage ${ }^{54}$ and extracellular dissolution with only ions entering hepatocytes ${ }^{55}$. Additionally NP dissolution can also occur within the NP preparations ${ }^{56}$ and in cell culture medium (ESI 2). The dynamic molecular responses detected could therefore reflect changing intracellular doses of metal ions. For future studies it is therefore recommended to obtain additional supporting data to estimate the intracellular doses, including time-resolved data on the bioavailable ion concentrations after exposure to the salts and cellular uptake rates of MPs, NPs and ions.

$\mathrm{CeO}_{2} \mathrm{NPs}$ led to relatively few significant alterations of transcription or metabolism compared with silver and zinc, reflecting both a decrease in molecular alterations with increasing $\mathrm{EC}_{20}$ concentrations (effective concentrations resulting in $20 \%$ cytotoxicity) and their low solubility (Table 1). A similar mild metabolomic and transcriptomic response to $\mathrm{CeO}_{2} \mathrm{NPs}$ was found 
by Taylor et al. ${ }^{12}$ in algae. Among the few significant alterations induced by the $\mathrm{CeO}_{2} \mathrm{NPs}$, only $\mathrm{CeO}_{2} \mathrm{NP}-\mathrm{A}$ (undoped $\mathrm{CeO}_{2} \mathrm{NP}$ ) exposure significantly increased metabolites putatively identified as cysteine and y-glutamylcysteine, potentially representing an adaptive response to oxidative stress by increased uptake and synthesis of these glutathione precursors. Because these changes were not observed after exposure to $\mathrm{CeO}_{2} \mathrm{NP}-\mathrm{C}$ ( $27 \% \mathrm{Zr}$-doped $\mathrm{CeO}_{2}$ $\mathrm{NPs}$ ) or $\mathrm{CeO}_{2} \mathrm{NP}-\mathrm{E}$ (78\% Zr-doped $\mathrm{CeO}_{2} \mathrm{NPs}$ ), this finding might indicate that modification of the surface redox state by Zr-doping increases the ability to scavenge ROS, resulting in a decreased induction of oxidative stress of the $\mathrm{CeO}_{2} \mathrm{NPs}$. Since all $\mathrm{CeO}_{2} \mathrm{NPs}$ showed very low cytotoxicity and exposure to only one of the four $\mathrm{CeO}_{2} \mathrm{NPs}$ showed any slight evidence of molecular responses to oxidative stress, the actual occurrence of oxidative stress, ROS or damage related to ROS was not further investigated.

$\mathrm{CeO}_{2}$ NP-A, $-\mathrm{C}$ and $-\mathrm{E}$ exposures all resulted in repression of ID family gene expression (Figure 4). Verstraelen et al. ${ }^{57}$ similarly found repression of ID2 transcription in A549 cells treated with $\mathrm{CeO}_{2}$ NPs. The ID, or Inhibitor of DNA binding, genes are binding partners of bHLH transcription factors and are involved in regulation of a wide variety of biological processes, including metastasis and vascularisation ${ }^{58} \cdot \mathrm{CeO}_{2}$ NP-NM212 elicited a different molecular response from the other $\mathrm{CeO}_{2} \mathrm{NPs}$, activating genes of the HIF1-alpha transcription factor network (Figure 4 ) by $24 \mathrm{~h}$. This response was also shared by the $6 \mathrm{~h} \mathrm{Ag}$ and $\mathrm{Zn}$ exposures, with additional induction of heme oxygenase (HMOX1). HIF1-alpha responsive genes are also commonly induced by several metal ions and particles ${ }^{59}$ and in cancer cells by ROS $^{60}$, leading to angiogenesis via VEGF. This finding is consistent with $\mathrm{CeO}_{2}$ and $\mathrm{Ag} \mathrm{NPs}$ inducing angiogenesis ${ }^{61,62}$.

\section{Conclusions}

A time series experiment was used to determine the similarity of A549 cellular responses following exposure to NPs and ions, as focussing on a single timepoint would have led to erroneous conclusions in the absence of internal dose measurements. The majority of molecular responses of $A 549$ cells to the Ag and Zn NPs, such as metallothionein induction, depletion of antioxidants, repressed DNA repair and induction of apoptosis, are similar to their responses to $\mathrm{Ag}$ and $\mathrm{Zn}$ ions, respectively, confirming that the modes of action of these NPs are largely mediated by dissolved metal ions rather than by the physical aspects of the NPs. Low toxicity $\mathrm{CeO}_{2} \mathrm{NPs}$ elicited only minor molecular responses. Of the four $\mathrm{CeO}_{2} \mathrm{NPs}$ tested, only $\mathrm{CeO}_{2}$ NP-A elicited any molecular changes indicative of oxidative stress.

\section{Acknowledgements}

The authors would like to thank Stephen Kissane and Fay Hughes for their assistance with RNA sequencing and Dr. Ralf Weber for bioinformatics assistance. The study has received funding from the European Union's Seventh Framework Programme under grant agreement $n^{\circ} 310451$ (NanoMILE) and the Netherlands Food and Consumer Product Safety Authority (NVWA). 


\section{References}

1. A. Nel, T. Xia, H. Meng, X. Wang, S. Lin, Z. Ji and H. Zhang. Nanomaterial toxicity testing in the 21st century: use of a predictive toxicological approach and high-throughput screening. Acc Chem Res, 2013, 46, 607-621.

2. V. De Matteis, M. A. Malvindi, A. Galeone, V. Brunetti, E. De Luca, S. Kote, P. Kshirsagar, S. Sabella, G. Bardi and P. P. Pompa. Negligible particle-specific toxicity mechanism of silver nanoparticles: the role of $\mathrm{Ag}+$ ion release in the cytosol. Nanomedicine, 2015, 11, 731-739.

3. J. Liu, X. Feng, L. Wei, L. Chen, B. Song and L. Shao. The toxicology of ion-shedding zinc oxide nanoparticles. Crit Rev Toxicol, 2016, 46, 348-384.

4. A. R. Gliga, S. Skoglund, I. O. Wallinder, B. Fadeel and H. L. Karlsson. Size-dependent cytotoxicity of silver nanoparticles in human lung cells: the role of cellular uptake, agglomeration and $\mathrm{Ag}$ release. Part Fibre Toxicol, 2014, 11, 11.

5. A. Ivask, K. Juganson, O. Bondarenko, M. Mortimer, V. Aruoja, K. Kasemets, I. Blinova, M. Heinlaan, V. Slaveykova and A. Kahru. Mechanisms of toxic action of $\mathrm{Ag}, \mathrm{ZnO}$ and $\mathrm{CuO}$ nanoparticles to selected ecotoxicological test organisms and mammalian cells in vitro: a comparative review. Nanotoxicology, 2014, 8 Suppl 1, 57-71.

6. I. Celardo, M. De Nicola, C. Mandoli, J. Z. Pedersen, E. Traversa and L. Ghibelli. Ce(3)+ ions determine redox-dependent anti-apoptotic effect of cerium oxide nanoparticles. ACS Nano, 2011, 5, 4537-4549.

7. K. M. Dunnick, R. Pillai, K. L. Pisane, A. B. Stefaniak, E. M. Sabolsky and S. S. Leonard. The Effect of Cerium Oxide Nanoparticle Valence State on Reactive Oxygen Species and Toxicity. Biol Trace Elem Res, 2015, 166, 96-107.

8. P. M. Costa and B. Fadeel. Emerging systems biology approaches in nanotoxicology: Towards a mechanism-based understanding of nanomaterial hazard and risk. Toxicol Appl Pharmacol, 2016, 299, 101-111.

9. S. Labib, A. Williams, C. L. Yauk, J. K. Nikota, H. Wallin, U. Vogel and S. Halappanavar. Nanorisk Science: application of toxicogenomics in an adverse outcome pathway framework for risk assessment of multi-walled carbon nanotubes. Part Fibre Toxicol, 2016, 13, 15.

10. J. M. Buescher and E. M. Driggers. Integration of omics: more than the sum of its parts. Cancer Metab, 2016, 4, 4.

11. S. Halappanavar, U. Vogel, H. Wallin and C. L. Yauk. Promise and peril in nanomedicine: the challenges and needs for integrated systems biology approaches to define health risk. Wiley Interdiscip Rev Nanomed Nanobiotechnol, 2018, 10, DOI: 10.1002/wnan.1465.

12. N. S. Taylor, R. Merrifield, T. D. Williams, J. K. Chipman, J. R. Lead and M. R. Viant. Molecular toxicity of cerium oxide nanoparticles to the freshwater alga Chlamydomonas reinhardtii is associated with supra-environmental exposure concentrations. Nanotoxicology, 2016, 10, 32-41.

13. S. Losert, N. von Goetz, C. Bekker, W. Fransman, S. W. Wijnhoven, C. Delmaar, K. Hungerbuhler and A. Ulrich. Human exposure to conventional and nanoparticle--containing sprays-a critical review. Environ Sci Technol, 2014, 48, 5366-5378. 
14. A. D. Maynard and E. D. Kuempel. Airborne Nanostructured Particles and Occupational Health. Journal of Nanoparticle Research, 2005, 7, 587-614.

15. K. Jensen, Kembouche, Y, Christiansen, E, Jacobsen, NR, Wallin, H Final protocol for producing suitable manufactured nanomaterial exposure media. The generic NANOGENOTOX dispersion protocol, 2011.

16. W. Slob. Dose-response modeling of continuous endpoints. Toxicol Sci, 2002, 66, 298-312.

17. C. L. Klein, Comero, S., Stahlmecke, B., Romazanov, J., Kuhlbusch, T. A. J., Van Doren, E., De Temmerman, P-J., Mast, J., Wick, P., Krug,H., Locoro, G., Hund-Rinke, K., Kördel, W., Friedrichs, S., Maier, G., Werner, J., Linsinger Th., Gawlik, B. M. , NM-Series of Representative Manufactured Nanomaterials NM-300 Silver Characterisation, Stability, Homogeneity, European Commission Joint Research Centre, Luxembourg, 2011.

18. C. Singh, Friedrichs, S., Levin, M., Birkedal, R., Jensen, K. A., Pojana, G., Wohlleben, W., Schulte, S. , Wiench, K. , Turney, T., Koulaeva, O. , Marshall, D., Hund-Rinke, K., Kördel, W., Van Doren, E., De Temmerman, P-J., Abi Daoud Francisco, M., Mast, J., Gibson, N., Koeber ,R., Linsinger, T., Klein C. L., NM-Series of Representative Manufactured Nanomaterials Zinc Oxide NM-110, NM-111, NM112, NM-113 Characterisation and Test Item Preparation, European Commission Joint Research Centre, Luxembourg, 2011.

19. C. Singh, S. Friedrichs, G. Ceccone, N. Gibson, K. Alstrup Jensen, M. Levin, H. Goenaga Infante, D. Carlander, K. Rasmussen., Cerium Dioxide, NM-211, NM-212, NM-213. Characterisation and test item preparation, Luxembourg, 2014.

20. A. D. Southam, T. G. Payne, H. J. Cooper, T. N. Arvanitis and M. R. Viant. Dynamic range and mass accuracy of wide-scan direct infusion nanoelectrospray fourier transform ion cyclotron resonance mass spectrometry-based metabolomics increased by the spectral stitching method. Anal Chem, 2007, 79, 4595-4602.

21. J. Zhang, M. A. Abdallah, T. D. Williams, S. Harrad, J. K. Chipman and M. R. Viant. Gene expression and metabolic responses of HepG2/C3A cells exposed to flame retardants and dust extracts at concentrations relevant to indoor environmental exposures. Chemosphere, 2016, 144, 19962003.

22. J. A. Kirwan, R. J. Weber, D. I. Broadhurst and M. R. Viant. Direct infusion mass spectrometry metabolomics dataset: a benchmark for data processing and quality control. Sci Data, 2014, 1, 140012.

23. A. D. Southam, R. J. Weber, J. Engel, M. R. Jones and M. R. Viant. A complete workflow for high-resolution spectral-stitching nanoelectrospray direct-infusion mass-spectrometry-based metabolomics and lipidomics. Nat Protoc, 2016, 12, 310-328.

24. R. J. Weber, A. D. Southam, U. Sommer and M. R. Viant. Characterization of isotopic abundance measurements in high resolution FT-ICR and Orbitrap mass spectra for improved confidence of metabolite identification. Anal Chem, 2011, 83, 3737-3743.

25. Y. Benjamini and Y. Hochberg. Controlling the False Discovery Rate: a Practical and Powerful Approach to Multiple Testing. J.R.Statist.Soc.B, 1995, 57, 289-300. 
26. M. I. Love, W. Huber and S. Anders. Moderated estimation of fold change and dispersion for RNAseq data with DESeq2. Genome Biol, 2014, 15, 550.

27. A. Kamburov, R. Cavill, T. M. Ebbels, R. Herwig and H. C. Keun. Integrated pathway-level analysis of transcriptomics and metabolomics data with IMPaLA. Bioinformatics, 2011, 27, 2917-2918.

28. D. S. Wishart, T. Jewison, A. C. Guo, M. Wilson, C. Knox, Y. Liu, Y. Djoumbou, R. Mandal, F. Aziat, E. Dong, S. Bouatra, I. Sinelnikov, D. Arndt, J. Xia, P. Liu, F. Yallou, T. Bjorndahl, R. Perez-Pineiro, R. Eisner, F. Allen, V. Neveu, R. Greiner and A. Scalbert. HMDB 3.0--The Human Metabolome Database in 2013. Nucleic Acids Res, 2013, 41, D801-807.

29. B. K. Gaiser, S. Hirn, A. Kermanizadeh, N. Kanase, K. Fytianos, A. Wenk, N. Haberl, A. Brunelli, W. G. Kreyling and V. Stone. Effects of silver nanoparticles on the liver and hepatocytes in vitro. Toxicol Sci, 2013, 131, 537-547.

30. M. M. Sthijns, W. Thongkam, C. Albrecht, B. Hellack, A. Bast, G. R. Haenen and R. P. Schins. Silver nanoparticles induce hormesis in A549 human epithelial cells. Toxicol In Vitro, 2017, 40, 223-233.

31. W. Thongkam, K. Gerloff, D. van Berlo, C. Albrecht and R. P. Schins. Oxidant generation, DNA damage and cytotoxicity by a panel of engineered nanomaterials in three different human epithelial cell lines. Mutagenesis, 2017, 32, 105-115.

32. J. Carrola, V. Bastos, I. Jarak, R. Oliveira-Silva, E. Malheiro, A. L. Daniel-da-Silva, H. Oliveira, C. Santos, A. M. Gil and I. F. Duarte. Metabolomics of silver nanoparticles toxicity in HaCaT cells: structure-activity relationships and role of ionic silver and oxidative stress. Nanotoxicology, 2016, 10, 1105-1117.

33. M. M. Cortese-Krott, M. Munchow, E. Pirev, F. Hessner, A. Bozkurt, P. Uciechowski, N. Pallua, K. D. Kroncke and C. V. Suschek. Silver ions induce oxidative stress and intracellular zinc release in human skin fibroblasts. Free Radic Biol Med, 2009, 47, 1570-1577.

34. M. Murata, P. Gong, K. Suzuki and S. Koizumi. Differential metal response and regulation of human heavy metal-inducible genes. J Cell Physiol, 1999, 180, 105-113.

35. Y. Li, T. Qin, T. Ingle, J. Yan, W. He, J. J. Yin and T. Chen. Differential genotoxicity mechanisms of silver nanoparticles and silver ions. Arch Toxicol, 2017, 91, 509-519.

36. S. F. Lin, H. Wei, D. Maeder, R. B. Franklin and P. Feng. Profiling of zinc-altered gene expression in human prostate normal vs. cancer cells: a time course study. J Nutr Biochem, 2009, 20, 10001012.

37. M. Simko, S. Tischler and M. O. Mattsson. Pooling and Analysis of Published in Vitro Data: A Proof of Concept Study for the Grouping of Nanoparticles. Int J Mol Sci, 2015, 16, 26211-26236.

38. P. Chairuangkitti, S. Lawanprasert, S. Roytrakul, S. Aueviriyavit, D. Phummiratch, K. Kulthong, P. Chanvorachote and R. Maniratanachote. Silver nanoparticles induce toxicity in A549 cells via ROSdependent and ROS-independent pathways. Toxicol In Vitro, 2013, 27, 330-338.

39. S. Aueviriyavit, D. Phummiratch and R. Maniratanachote. Mechanistic study on the biological effects of silver and gold nanoparticles in Caco-2 cells--induction of the Nrf2/HO-1 pathway by high concentrations of silver nanoparticles. Toxicol Lett, 2014, 224, 73-83.

40. S. O. Simmons, C. Y. Fan, K. Yeoman, J. Wakefield and R. Ramabhadran. NRF2 Oxidative Stress Induced by Heavy Metals is Cell Type Dependent. Curr Chem Genomics, 2011, 5, 1-12. 
41. L. M. Faddah, N. A. Abdel Baky, N. M. Al-Rasheed, N. M. Al-Rasheed, A. J. Fatani and M. Atteya. Role of quercetin and arginine in ameliorating nano zinc oxide-induced nephrotoxicity in rats. BMC Complement Altern Med, 2012, 12, 60.

42. X. Yang, H. Shao, W. Liu, W. Gu, X. Shu, Y. Mo, X. Chen, Q. Zhang and M. Jiang. Endoplasmic reticulum stress and oxidative stress are involved in $\mathrm{ZnO}$ nanoparticle-induced hepatotoxicity. Toxicol Lett, 2015, 234, 40-49.

43. P. Dubey, I. Matai, S. U. Kumar, A. Sachdev, B. Bhushan and P. Gopinath. Perturbation of cellular mechanistic system by silver nanoparticle toxicity: Cytotoxic, genotoxic and epigenetic potentials. Adv Colloid Interface Sci, 2015, 221, 4-21.

44. R. Foldbjerg, D. A. Dang and H. Autrup. Cytotoxicity and genotoxicity of silver nanoparticles in the human lung cancer cell line, A549. Arch Toxicol, 2011, 85, 743-750.

45. J. Heim, E. Felder, M. N. Tahir, A. Kaltbeitzel, U. R. Heinrich, C. Brochhausen, V. Mailander, W. Tremel and J. Brieger. Genotoxic effects of zinc oxide nanoparticles. Nanoscale, 2015, 7, 89318938.

46. Y. S. Lee, D. W. Kim, Y. H. Lee, J. H. Oh, S. Yoon, M. S. Choi, S. K. Lee, J. W. Kim, K. Lee and C. W. Song. Silver nanoparticles induce apoptosis and G2/M arrest via PKCzeta-dependent signaling in A549 lung cells. Arch Toxicol, 2011, 85, 1529-1540.

47. R. Y. Prasad, J. K. McGee, M. G. Killius, D. A. Suarez, C. F. Blackman, D. M. DeMarini and S. O. Simmons. Investigating oxidative stress and inflammatory responses elicited by silver nanoparticles using high-throughput reporter genes in HepG2 cells: effect of size, surface coating, and intracellular uptake. Toxicol In Vitro, 2013, 27, 2013-2021.

48. H. Kocdor, H. Ates, S. Aydin, R. Cehreli, F. Soyarat, P. Kemanli, D. Harmanci, H. Cengiz and M. A. Kocdor. Zinc supplementation induces apoptosis and enhances antitumor efficacy of docetaxel in non-small-cell lung cancer. Drug Des Devel Ther, 2015, 9, 3899-3909.

49. P. J. Shiny, Mukherjee, A., Chandrasekaran, N. DNA damage and mitochondria-mediated apoptosis of A549 lung carcinoma cells induced by biosynthesised silver and platinum nanoparticles. RSC Advances, 2016, 6, 27775-27787.

50. X. Lai, Y. Wei, H. Zhao, S. Chen, X. Bu, F. Lu, D. Qu, L. Yao, J. Zheng and J. Zhang. The effect of $\mathrm{Fe} 2 \mathrm{O} 3$ and $\mathrm{ZnO}$ nanoparticles on cytotoxicity and glucose metabolism in lung epithelial cells. $J$ Appl Toxicol, 2015, 35, 651-664.

51. G. T. Ankley, R. S. Bennett, R. J. Erickson, D. J. Hoff, M. W. Hornung, R. D. Johnson, D. R. Mount, J. W. Nichols, C. L. Russom, P. K. Schmieder, J. A. Serrrano, J. E. Tietge and D. L. Villeneuve. Adverse outcome pathways: a conceptual framework to support ecotoxicology research and risk assessment. Environ Toxicol Chem, 2010, 29, 730-741.

52. T. Miyayama and M. Matsuoka. Involvement of Iysosomal dysfunction in silver nanoparticleinduced cellular damage in A549 human lung alveolar epithelial cells. J Occup Med Toxicol, 2016, $11,1$.

53. Y. Arai, T. Miyayama and S. Hirano. Difference in the toxicity mechanism between ion and nanoparticle forms of silver in the mouse lung and in macrophages. Toxicology, 2015, 328, 84-92. 
54. T. Xia, M. Kovochich, M. Liong, L. Madler, B. Gilbert, H. Shi, J. I. Yeh, J. I. Zink and A. E. Nel. Comparison of the mechanism of toxicity of zinc oxide and cerium oxide nanoparticles based on dissolution and oxidative stress properties. ACS Nano, 2008, 2, 2121-2134.

55. M. Chevallet, B. Gallet, A. Fuchs, P. H. Jouneau, K. Um, E. Mintz and I. Michaud-Soret. Metal homeostasis disruption and mitochondrial dysfunction in hepatocytes exposed to sub-toxic doses of zinc oxide nanoparticles. Nanoscale, 2016, 8, 18495-18506.

56. C. Beer, R. Foldbjerg, Y. Hayashi, D. S. Sutherland and H. Autrup. Toxicity of silver nanoparticles nanoparticle or silver ion? Toxicol Lett, 2012, 208, 286-292.

57. S. Verstraelen, S. Remy, E. Casals, P. De Boever, H. Witters, A. Gatti, V. Puntes and I. Nelissen. Gene expression profiles reveal distinct immunological responses of cobalt and cerium dioxide nanoparticles in two in vitro lung epithelial cell models. Toxicol Lett, 2014, 228, 157-169.

58. F. Ling, B. Kang and X. H. Sun. Id proteins: small molecules, mighty regulators. Curr Top Dev Biol, 2014, 110, 189-216.

59. R. M. Harris, T. D. Williams, R. H. Waring and N. J. Hodges. Molecular basis of carcinogenicity of tungsten alloy particles. Toxicol Appl Pharmacol, 2015, 283, 223-233.

60. L. Z. Liu, X. W. Hu, C. Xia, J. He, Q. Zhou, X. Shi, J. Fang and B. H. Jiang. Reactive oxygen species regulate epidermal growth factor-induced vascular endothelial growth factor and hypoxiainducible factor-1alpha expression through activation of AKT and P70S6K1 in human ovarian cancer cells. Free Radic Biol Med, 2006, 41, 1521-1533.

61. S. Das, S. Singh, J. M. Dowding, S. Oommen, A. Kumar, T. X. Sayle, S. Saraf, C. R. Patra, N. E. Vlahakis, D. C. Sayle, W. T. Self and S. Seal. The induction of angiogenesis by cerium oxide nanoparticles through the modulation of oxygen in intracellular environments. Biomaterials, 2012, 33, 7746 7755.

62. K. Kang, D. H. Lim, I. H. Choi, T. Kang, K. Lee, E. Y. Moon, Y. Yang, M. S. Lee and J. S. Lim. Vascular tube formation and angiogenesis induced by polyvinylpyrrolidone-coated silver nanoparticles. Toxicol Lett, 2011, 205, 227-234. 


\section{Supporting Information}

\section{ESI 1: Cytotoxicity of tested particles to A549 cells}

Table S1: Overview of $\mathrm{EC}_{20}$ and confidence interval for dose calculation.

\begin{tabular}{|c|c|c|c|c|c|}
\hline Test material & $\begin{array}{c}\mathrm{EC}_{20} \\
\text { Exponential } \\
\text { model } \mu \mathrm{g} / \mathrm{mL}\end{array}$ & $\begin{array}{c}\text { Confidence } \\
\text { interval } \mu \mathrm{g} / \mathrm{mL}\end{array}$ & $\begin{array}{c}\mathrm{EC}_{20} \\
\text { Hill model } \\
\mu \mathrm{g} / \mathrm{mL}\end{array}$ & $\begin{array}{c}\text { Confidence } \\
\text { interval } \mu \mathrm{g} / \mathrm{mL}\end{array}$ & $\begin{array}{c}\text { Selected Dose } \\
\mu \mathrm{g} / \mathrm{mL}\end{array}$ \\
\hline $\mathrm{CeO}_{2} \mathrm{NP}-\mathrm{A}$ & n.d. ${ }^{1}$ & & n.d. & & 128 \\
\hline $\mathrm{CeO}_{2} \mathrm{NP}-\mathrm{C}$ & n.d. & & n.d. & & 128 \\
\hline $\mathrm{CeO}_{2} \mathrm{NP}-\mathrm{E}$ & n.d. & & n.d. & & 128 \\
\hline $\mathrm{CeO}_{2}$ NP-NM212 & n.d. & & n.d. & & 128 \\
\hline ZnO NP-NM110 & 29.4 & $22.4-37.3$ & 33.9 & $29.2-38.9$ & 31.6 \\
\hline ZnO NP-NM111 & 23.3 & $16.9-30.6$ & 42.6 & $37.9-51.4$ & 32.9 \\
\hline ZnO MP & 57.6 & $54.9-61.6$ & 57 & $54.3-61.1$ & 57.3 \\
\hline $\mathrm{ZnCl}_{2}$ & 19.7 & $14.2-26.1$ & 29.4 & $27.7-31.4$ & 24.5 \\
\hline Ag NP-NM300K & 37.9 & $33.5-50.1$ & 39.3 & $34.0-49.7$ & 38.6 \\
\hline $\mathrm{Ag} \mathrm{MP}$ & n.d. & & n.d. & & 128 \\
\hline $\mathrm{AgNO}_{3}$ & 9.6 & $2.6-12.8$ & 10.1 & $5.2-11.3$ & 9.8 \\
\hline
\end{tabular}

${ }^{1}$ n.d. = dose could not be determined, highest dose (i.e. $128 \mu \mathrm{g} / \mathrm{mL}$ ) was used for the omics experiments.

2 Selected Dose $=$ the highest concentration tested or the average of the $\mathrm{EC}_{20} \mathrm{~s}$ of the Exponential and Hill model.

\section{ESI 2: Dissolution of NPs and MPs in cell culture medium using ICP-OES}

The NP and MP dispersions in cell culture medium ( $\mathrm{pH}$ 7.1) were prepared according to the same procedure as used for the preparation of the dispersions for the omics study. Dissolution of the NPs and MPs in cell culture medium was measured at various time intervals $(0,6$, and $24 \mathrm{~h})$. At each time interval, $10 \mathrm{~mL}$ of the dispersion was transferred to $3 \mathrm{kDa}$ Millipore centrifuge tubes (Satorius, UK) and subjected to centrifugal ultrafiltration at $5000 \mathrm{~g}$ for 50 minutes to separate the dissolved fraction. Nitric acid (ICP grade) was added to the filtrate samples to a final concentration of $2 \%$ and samples were subsequently analysed using a Perkin Elmer ICP-OES Optima 8000 platform in order to assess how much, if any, dissolution of the NMs or MPs had occurred. Each sample was prepared and analysed in triplicate. Prior to acquisition the torch was aligned (radially and axially) using the $\mathrm{Mn}$ wavelengths and calibration curves were constructed using standard solutions of each element prepared by serial dilutions of $1000 \mathrm{ppm}$ in $2 \%$ nitric acid. A calibration curve with $R^{2}$ value of $>0.999$ was deemed sufficient for analysis.

The concentrations of ions detected at each time point are presented in Table S2 below. Interestingly, the dissolved concentration from ZnO NPs determined at 24 hours in complete cell culture medium were higher than the dissolved concentrations determined at $\mathrm{pH} 7$ in DI water (Papadiamantis et al., manuscript in preparation), consistent with the known 
affinity of $\mathrm{Zn}^{2+}$ for biological components (protein, lysosomes etc.) $)^{1}$ and its known tendency to form complexes with organic components in the long term ${ }^{2-4}$. Clearly, this would also be the case for the $\mathrm{Zn}^{2+}$ ions released from the ionic control, which would be similarly bound to biomolecules.

Table S2: Ion concentrations in cell culture medium after 0,6 and 24 hours.

\begin{tabular}{|c|c|c|c|c|}
\hline \multirow[b]{2}{*}{$\begin{array}{l}\text { Incubation (hours) } \rightarrow \\
\text { Test material } \downarrow\end{array}$} & \multirow{2}{*}{$\begin{array}{c}\begin{array}{c}\text { Initial MP or } \\
\text { NM concentration } \\
(\mathrm{mg} / \mathrm{L})\end{array} \\
0\end{array}$} & \multicolumn{3}{|c|}{ Ion concentration $(\mu \mathrm{g} / \mathrm{mL})$} \\
\hline & & 0 & 6 & 24 \\
\hline Ag NP-NM300K & 38.6 & $0.075+/-0.01$ & $0.093+/-0.03$ & $0.040+/-0.003$ \\
\hline $\mathrm{Ag} \mathrm{MP}$ & 128 & $0.005+/-0.0002$ & $0.004+/-0.0001$ & $0.005+/-0.0004$ \\
\hline ZnO NP-NM110 & 15 & $1.401+/-0.06$ & $1.378+/-0.07$ & $1.411+/-0.01$ \\
\hline ZnO NP- NM 111 & 10 & $0.439+/-0.02$ & $0.899+/-0.07$ & $0.989+/-0.03$ \\
\hline ZnO MP & 30 & $1.323+/-0.22$ & $1.338+/-0.10$ & $1.461+/-0.03$ \\
\hline $\mathrm{CeO}_{2} \mathrm{NP}-\mathrm{A}$ & 128 & $<\mathrm{LOD}$ & $<\mathrm{LOD}$ & $<\mathrm{LOD}$ \\
\hline $\mathrm{CeO}_{2} \mathrm{NP}-\mathrm{C}$ & 128 & $<\mathrm{LOD}$ & $<\mathrm{LOD}$ & $<\mathrm{LOD}$ \\
\hline $\mathrm{CeO}_{2} \mathrm{NP}-\mathrm{E}$ & 128 & $<\mathrm{LOD}$ & $<\mathrm{LOD}$ & $<\mathrm{LOD}$ \\
\hline $\mathrm{CeO}_{2}$ NP-NM212 & 128 & $<\mathrm{LOD}$ & $<\mathrm{LOD}$ & $<\mathrm{LOD}$ \\
\hline
\end{tabular}

References:

${ }^{1}$ V. Frazzini, E. Rockabrand, E. Mocchegiani and S. L. Sensi. Oxidative stress and brain aging: is zinc the link? Biogerontology, 2006, 7, 307-314.

2 M. Li, L. Zhu and D. Lin. Toxicity of ZnO nanoparticles to Escherichia coli: mechanism and the influence of medium components. Environ Sci Technol, 2011, 45, 1977-1983.

${ }^{3}$ R. B. Reed, D. A. Ladner, C. P. Higgins, P. Westerhoff and J. F. Ranville. Solubility of nano-zinc oxide in environmentally and biologically important matrices. Environ Toxicol Chem, 2012, 31, 93-99.

${ }^{4}$ T. Xia, M. Kovochich, M. Liong, L. Madler, B. Gilbert, H. Shi, J. I. Yeh, J. I. Zink and A. E. Nel. Comparison of the mechanism of toxicity of zinc oxide and cerium oxide nanoparticles based on dissolution and oxidative stress properties. ACS Nano, 2008, 2, 2121-2134.

\section{ESI 3: Methods}

\section{Omics Analyses}

\section{S1.1 Extraction of metabolites and lipids}

Deep frozen cells were harvested by adding $400 \mu \mathrm{L} 80 \%$ methanol (pre-cooled on dry ice) into each well. Cells were scraped from each well on dry ice and were transferred into a 1.8 $\mathrm{mL}$ glass vial which was pre-filled with $640 \mu \mathrm{L}$ pre-cooled chloroform and $416 \mu \mathrm{L} \mathrm{H} 2 \mathrm{O} .400 \mu \mathrm{L}$ $80 \%$ methanol (pre-cooled on dry ice) was used to wash each well and the wash solution was transferred into the same glass vial. After adjusting the ratio of methanol:chloroform:water $(\mathrm{v} / \mathrm{v} / \mathrm{v})$ to 1:1:0.9, each glass vial was vortexed for $30 \mathrm{~s}$ twice, at $30 \mathrm{~s}$ intervals. The glass vials were then cooled on dry ice for 10 mins before centrifugation for 10 mins at 3000-g at -9 ${ }^{\circ} \mathrm{C}$, which separated the mixture into two phases (upper polar phase and lower non-polar phase). $300 \mu \mathrm{L}$ aliquots of the polar phase were transferred into clean $1.5 \mathrm{~mL}$ Eppendorf 
tubes and then dried in a speed vac concentrator (Thermo Savant, Holbrook, NY) for $4 \mathrm{~h}$. $300 \mu \mathrm{L}$ aliquots of the non-polar phase were transferred into clean $1.8 \mathrm{~mL}$ glass vials and dried under a stream of nitrogen for 5 mins. All dried samples were then frozen at $-80^{\circ} \mathrm{C}$ until analysis.

\section{S1.2 Direct infusion mass spectrometry (DIMS)}

The DIMS analysis method used was similar to the one reported previously (Southam et al. 2007; Zhang et al. 2016). The dried polar or non-polar extracts were re-suspended in 80 $\mu \mathrm{L}$ 80:20 (v/v) methanol:water (HPLC grade) with $0.25 \%$ formic acid (for positive ion mode analysis of polar extracts) or $80 \mu \mathrm{L} 2: 1$ methanol:chloroform with $5 \mathrm{mM}$ ammonium acetate (for negative ion mode analysis of lipids). After centrifugation at $22000 \mathrm{~g}, 4^{\circ} \mathrm{C}$ for $10 \mathrm{~min}, 10$ $\mu \mathrm{L}$ supernatant of each sample was loaded into each of four well of a 384-well plate and then analysed (in quadruplicate) using direct infusion mass spectrometry ( $Q$ Exactive, Thermo Fisher Scientific, Germany) in positive ion mode (for polar metabolomics) or negative ion mode (for lipidomics), utilising a Triversa nanoelectrospray ion source (Advion Biosciences, Ithaca, NY, USA).

\section{S1.3 Metabolomics Data Processing}

Mass spectra were recorded using the selected ion monitoring (SIM) stitching approach from m/z 50-620 (for polar metabolomics) or from m/z 50-1020 (for lipidomics) and then processed using custom-written Matlab scripts as previously reported (Kirwan et al. 2014; Southam et al. 2016). In brief, only mass spectral peaks with a signal-to-noise ratio exceeding 3.5 were retained. For each sample the combination of three of the four technical replicates that has the smallest relative standard deviation (RSD) was selected, which were aligned with a $2 \mathrm{ppm}$ mass range and filtered into a single peak list (with only those peaks present in $\geq 2$ of the 3 spectra retained). Each filtered peak list (one per sample) was then further aligned with a $2 \mathrm{ppm}$ mass range, and filtered to retain only those peaks that were present in $80 \%$ of all biological samples in the entire dataset (this filtering step was reduced to $50 \%$ for the $\mathrm{Zn}$ and $\mathrm{Ag}$ lipidomics data). Peaks in the blank samples were removed from the sample spectra. The resulting matrices of peak intensities (termed "DIMS dataset") were probabilistic-quotient normalised (PQN) and intensity-drift corrected using a Quality ControlRobust Spline Correction (QC-RSC) algorithm. Finally, the missing values were imputed using the k-nearest neighbours (KNN) algorithm. For multivariate analysis, generalized log (Glog) transformation of the DIMS dataset was also performed.

\section{S1.4 RNA seq gene expression profiling}

Total RNA was extracted from A549 cells using a micro RNeasy Kit (Qiagen, Crawley, UK) according to the manufacturer's protocol. RNA was quantified with a NanoDrop 8000 spectrophotometer (Thermo Scientific, Waltham, MA), and the integrity of RNA was evaluated with a Agilent 2200 Tapestation (Agilent Technologies, Santa Clara, CA). Only RNA with 
integrity numbers (RINs) greater than 7.0 were used for subsequent RNA-seq experiments. All RNA libraries were produced using the Biomek FxP (Beckman Coulter A31842) with Ultra Directional RNA Library Prep Kit (New England Biolab E7420L) and NEBnext Multiplex Oligos for Illumina Dual Index Primers (New England Biolabs E7600S), using provided protocols and 500 ng of total RNA. Constructed libraries were assessed for quality using the Tapestation 2200 (Agilent G2964AA) with High Sensitivity D1000 DNA screentape (5067-5584), and quantified using Kapa Library Quantification Kit (Kapa Biosystems KK4824) on an AriaMx Realtime PCR System (Agilent G8830A). Multiplex library clustering and sequencing was performed upon the HiSeq2500 with HiSeq Rapid Cluster Kit v2 (Illumina GD-402-4002) at $12 \mathrm{pM}$ library concentration with $10 \%$ PhiX Control v3 spiked in (Illumina FC-110-3001). The sequencing run was carried out using HiSeq Rapid SBS Kit v2 (Illumina FC-402-4021).

\section{S1.5 RNA seq Data Processing}

The BCL files were converted to FASTQ format using Illumina bcl2fastq conversion software (v1.8.4). Sequences were then trimmed using Trimmomatic (v0.36). FastQC (v0.11.2) was utilised to assess the data quality, and the $Q C$ results were summarised with MultiQC (v0.8). Five low quality samples were identified and removed accordingly. The remaining FASTQ files were aligned to the GENCODE human transcript sequences (release 25, GRCh38.p7) using Bowtie2 (v2.3.0). The resulting SAM data were converted into BED format using SAMtools (v1.3.1) and bamToBed (v2.19.1). Finally, the RNA read counts were extracted from the BED files with a Python script. To provide gene-level analysis, the RNA reads were collapsed to the counts of their coding genes. The gene annotation information was retrieved from the Ensembl database (release 87).

\section{S1.6 Omics Data Analysis}

Lipidomics and polar metabolomics peak intensity data were visualised in Genespring (v7.3.1; Agilent) and putative annotations were added using MI-Pack (Weber et al. 2011). ANOVAs and t-tests were performed in Genespring using multiple testing corrections (Benjamini and Hochberg 1995) for false discovery rate corrected $p$-values (FDR) of $<0.05$. Transcriptional data was mapped by gene. DESeq2 (Love et al. 2014) and used for differential expression analysis with a $\mathrm{q}<0.05$ cut-off. Genespring was used to generate principal components analysis (PCA) scores plots from all omics datasets. Combined gene and metabolite pathway over-representation analyses were performed with IMPaLA (Kamburov et al. 2011), using gene identifiers and all Human Metabolite Database (HMDB) identifiers (Wishart et al. 2013) for each peak identified as significantly altered as input lists. The background sets were all detected transcripts and all identifiers annotated to detected lipid and polar metabolite peaks, respectively. Comparative pathway analyses were performed with Ingenuity Pathway Analysis (IPA; Qiagen) on combined sets of genes, lipids and polar metabolite identifiers using the mean fold change and FDR for each exposure group versus time-matched controls; analyses used only those identifiers with FDR<0.05. Raw data and experimental details are archived at ArrayExpress for transcriptomics (accession number E-MTAB-5734). 


\section{References}

Benjamini Y, Hochberg Y (1995) Controlling the False Discovery Rate: a Practical and Powerful Approach to Multiple Testing. JRStatistSocB 57(1):289-300

Kamburov A, Cavill R, Ebbels TM, Herwig R, Keun HC (2011) Integrated pathway-level analysis of transcriptomics and metabolomics data with IMPaLA. Bioinformatics 27(20):2917-8 doi:10.1093/ bioinformatics/btr499

Kirwan JA, Weber RJ, Broadhurst DI, Viant MR (2014) Direct infusion mass spectrometry metabolomics dataset: a benchmark for data processing and quality control. Sci Data 1:140012 doi:10.1038/ sdata.2014.12

Love MI, Huber W, Anders S (2014) Moderated estimation of fold change and dispersion for RNA-seq data with DESeq2. Genome Biol 15(12):550 doi:10.1186/s13059-014-0550-8

Southam AD, Weber RJ, Engel J, Jones MR, Viant MR (2016) A complete workflow for high-resolution spectral-stitching nanoelectrospray direct-infusion mass-spectrometry-based metabolomics and lipidomics. Nat Protoc 12(2):310-328 doi:10.1038/nprot.2016.156

Weber RJ, Southam AD, Sommer U, Viant MR (2011) Characterization of isotopic abundance measurements in high resolution FT-ICR and Orbitrap mass spectra for improved confidence of metabolite identification. Anal Chem 83(10):3737-43 doi:10.1021/ac2001803

Wishart DS, Jewison T, Guo AC, et al. (2013) HMDB 3.0--The Human Metabolome Database in 2013. Nucleic Acids Res 41(Database issue):D801-7 doi:10.1093/nar/gks1065

\section{ESI 4: Univariate analyses of omics data}

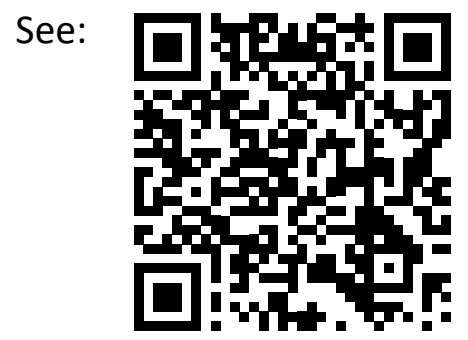

\section{ESI 5: PCA scores plots of omics data}

Principal components analysis (PCA) scores plots of transcriptomic, lipidomic and metabolomic data derived from exposure of A549 cells to silver, zinc and $\mathrm{CeO}_{2}$ nanoparticles, microparticles and/or ions. 
Silver 1 hour

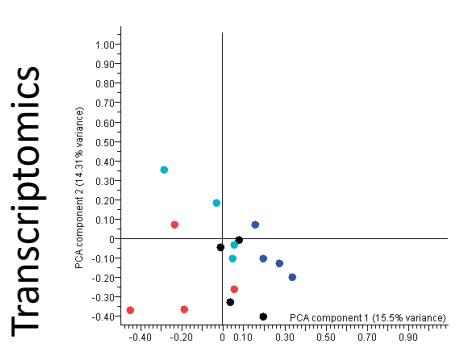

6 hours

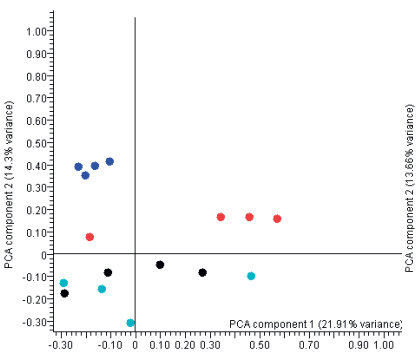

24 hours

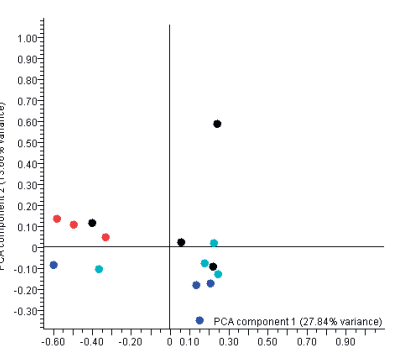

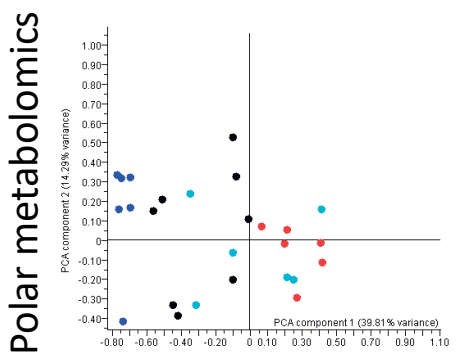
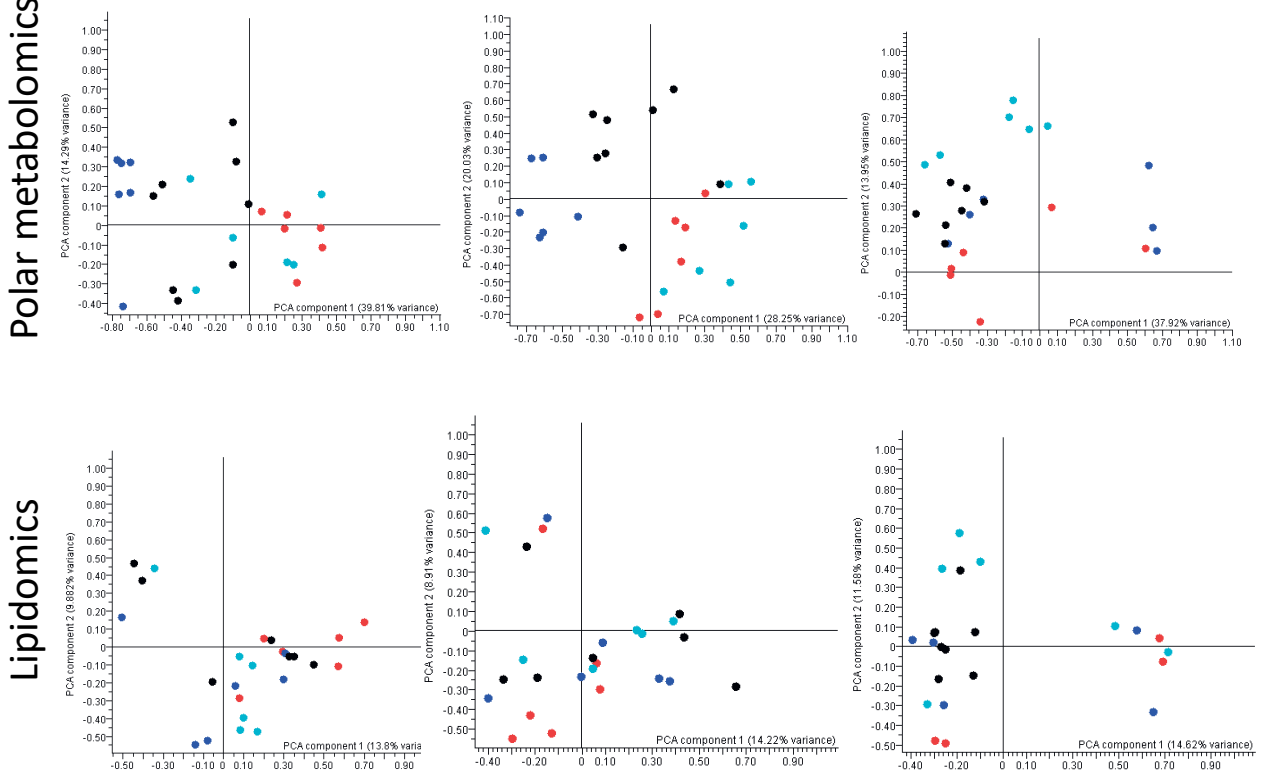

control samples are shown in black, Ag NP-NM300K treated in red,

Ag MP in cyan,

$\mathrm{Ag}^{+}$in blue. 


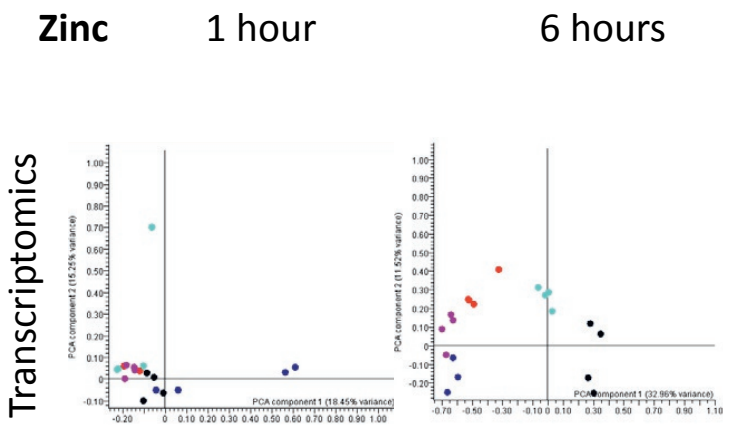

24 hours
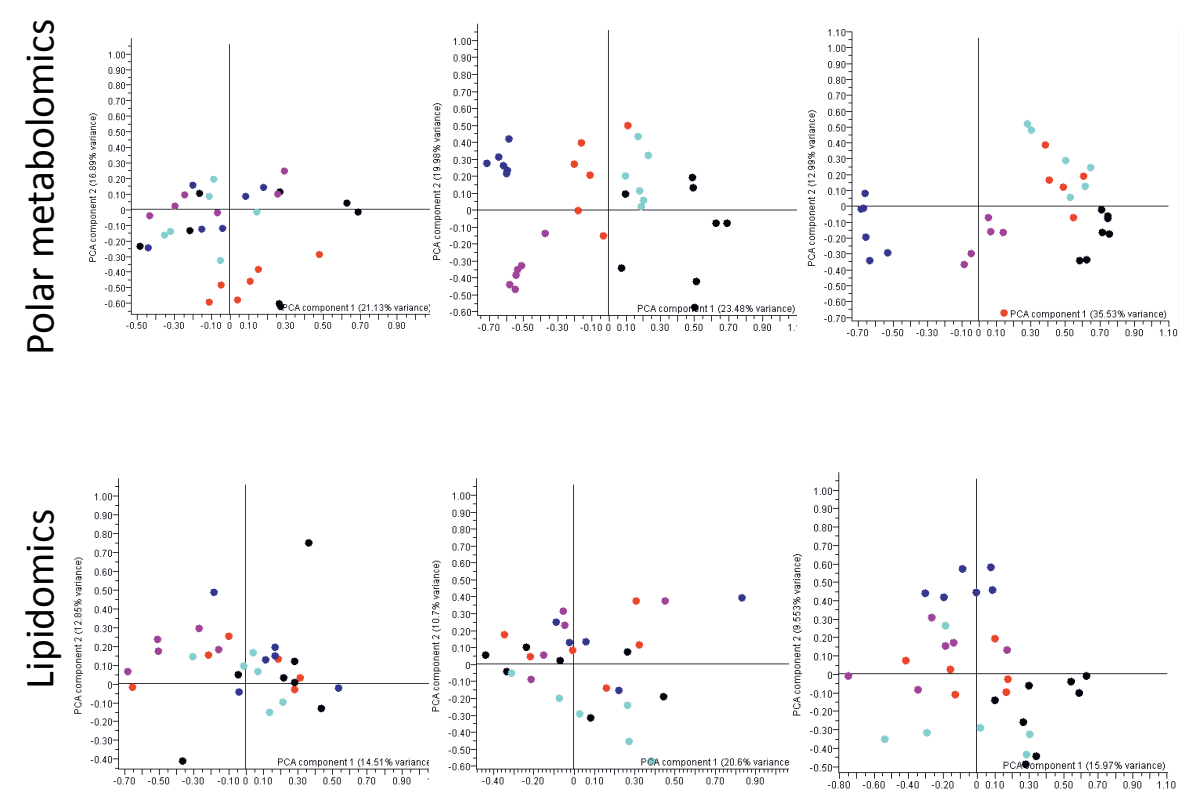

control samples are shown in black,

ZnO NP-NM110 treated in red,

ZnO NP-NM111 in cyan,

$\mathrm{Zn}_{2+}$ in blue

$\mathrm{ZnO} \mathrm{MP}$ in pink. 

$\mathrm{CeO}_{2} 1$ hour
6 hours
24 hours
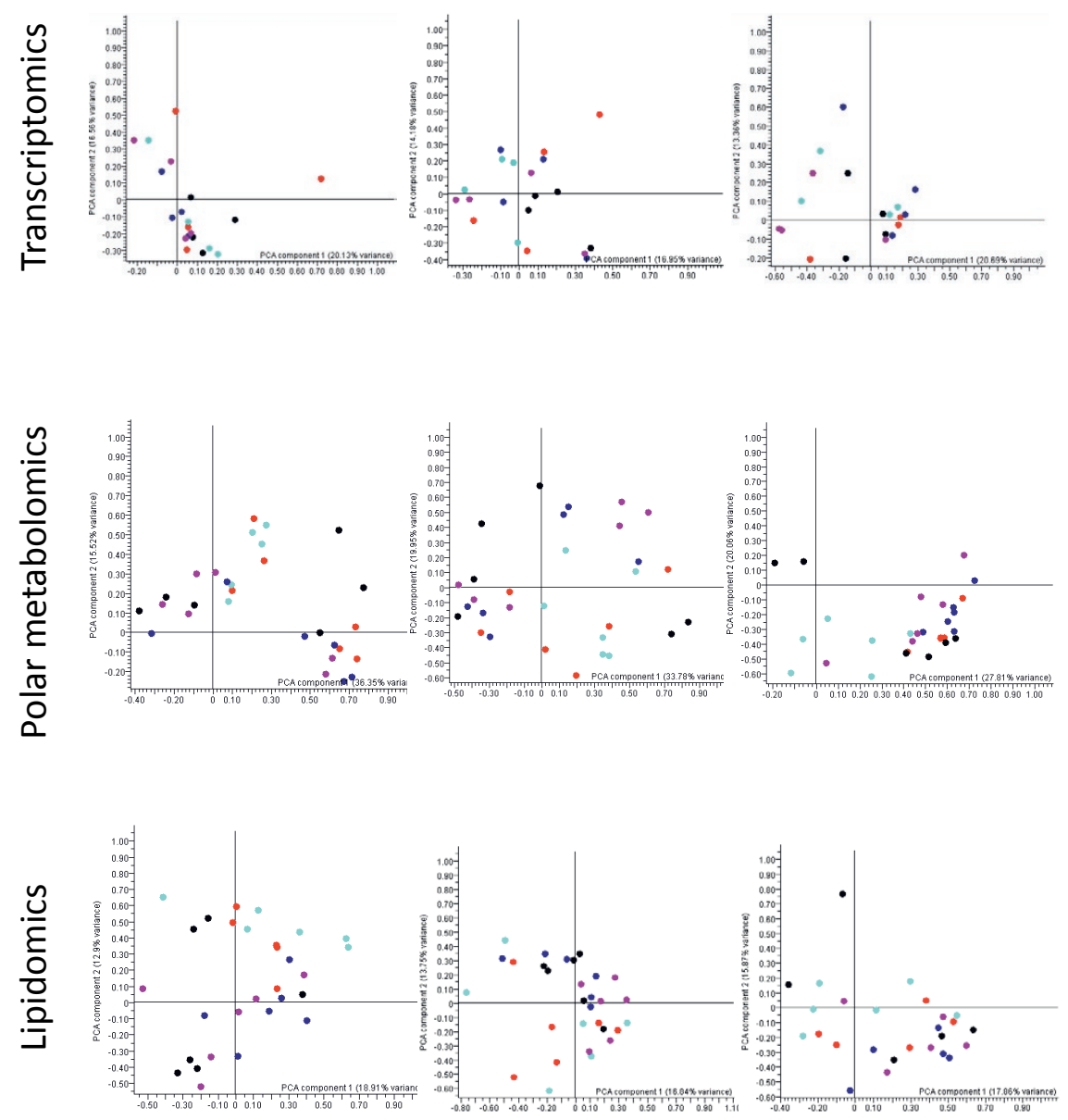

control samples are shown in black, $\mathrm{CeO}_{2}$ NP-A treated in red, $\mathrm{CeO}_{2}$ NP-C in cyan, $\mathrm{CeO}_{2}$ NP-E in blue $\mathrm{CeO}_{2}$ NP-NM212 in pink.

ESI 6: IMPaLA pathway analysis of omics data See:

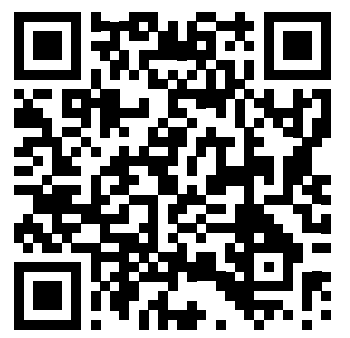

ESI 7: Methods

See:

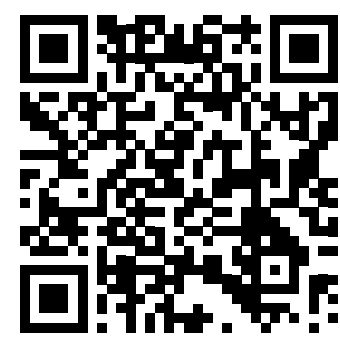




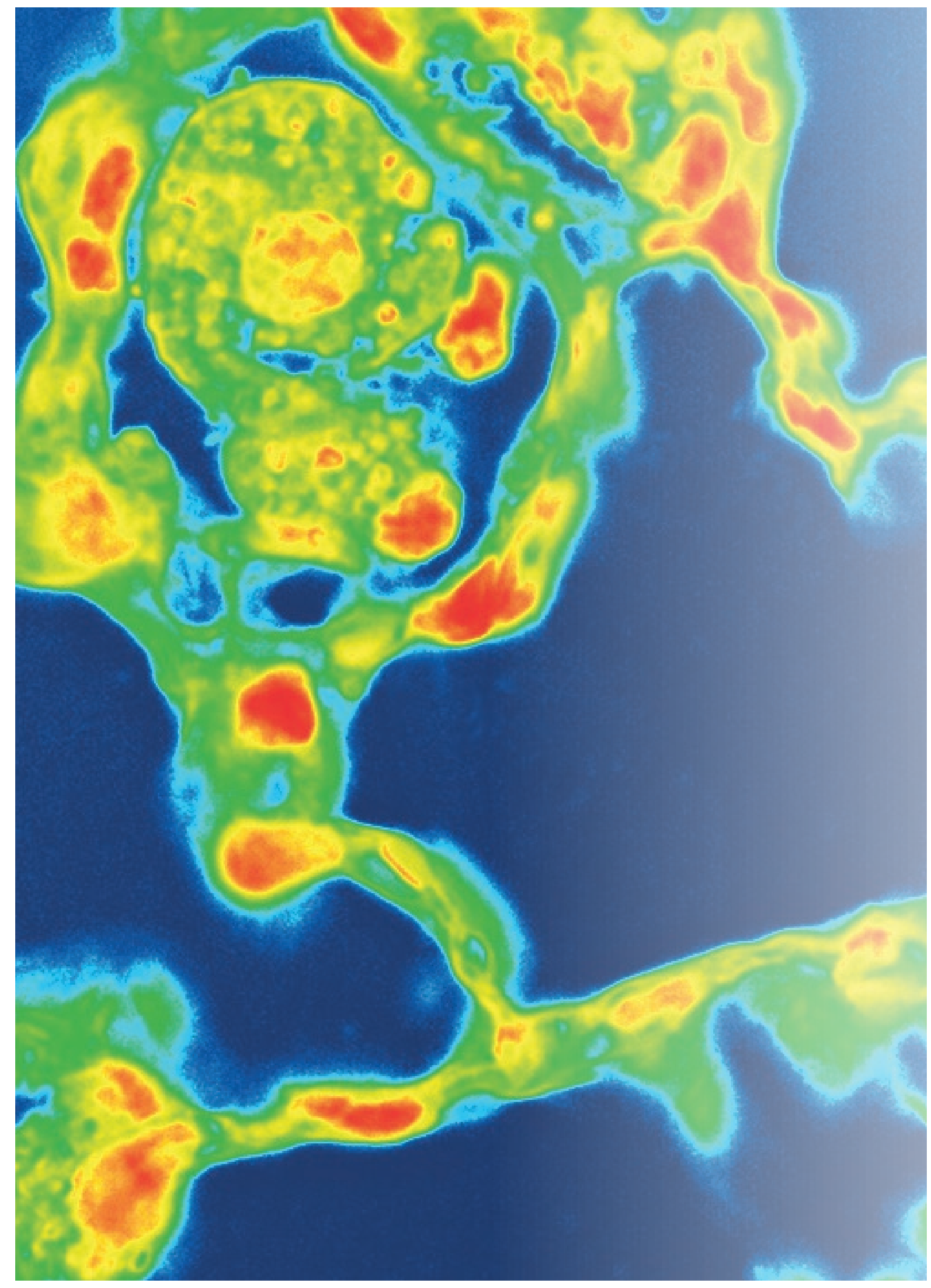




\section{Chapter 5}

\section{Role of chemical composition and}

redox modification of poorly soluble nanomaterials on their ability to enhance allergic airway sensitisation in mice

Susan Dekkers' , James G. Wagner², Rob J. Vandebriel' , Elyse A. Eldridge², Selina V.Y. Tang ${ }^{3}$, Mark R. Miller ${ }^{4}$, Isabella Römer ${ }^{5}$, Wim H. de Jong' Jack R. Harkema ${ }^{2}$ and Flemming R. Cassee ${ }^{1,6}$

Particle and Fibre Toxicology 16: 39 (2019)

1 National Institute for Public Health and the Environment, Bilthoven, the Netherlands,

2 Department of Pathobiology and Diagnostic Investigation, Michigan State University, East Lansing, Ml, United States of America,

3 Promethean Particles Ltd, Nottingham, United Kingdom,

${ }^{4}$ Centre for Cardiovascular Science, University of Edinburgh, Edinburgh, United Kingdom,

5 School of Geography, Earth and Environmental Sciences, University of Birmingham, Birmingham, United Kingdom.

${ }^{6}$ Institute for Risk Assessment Sciences, Utrecht University, Utrecht, the Netherlands 


\begin{abstract}
Background: Engineered nanoparticles (NPs) have been shown to enhance allergic airways disease in mice. However, the influence of the different physicochemical properties of these particles on their adjuvant properties is largely unknown. Here we investigate the effects of chemical composition and redox activity of poorly soluble NPs on their adjuvant potency in a mouse model of airway hypersensitivity.
\end{abstract}

Results: NPs of roughly similar sizes with different chemical composition and redox activity, including $\mathrm{CeO}_{2}, \mathrm{Zr}$-doped $\mathrm{CeO}_{2}, \mathrm{CO}_{3} \mathrm{O}_{4^{\prime}}$ Fe-doped $\mathrm{Co}_{3} \mathrm{O}_{4}$ (using $\mathrm{Fe}_{2} \mathrm{O}_{3}$ or $\mathrm{Fe}_{3} \mathrm{O}_{4}$ ) and $\mathrm{TiO}_{2} \mathrm{NPs}$, all showed adjuvant activity. OVA induced immune responses following intranasal exposure of BALB/c mice to $0.02 \%$ OVA in combination with $200 \mu \mathrm{g}$ NPs during sensitization (on day 1 , 3,6 and 8 ) and $0.5 \%$ OVA only during challenge (day 22, 23 and 24) were more pronounced compared to the same OVA treatment regime without NPs. Changes in OVA-specific IgE and IgG1 plasma levels, differential cell count and cytokines in bronchoalveolar lavage fluid (BALF), and histopathological detection of mucosa cell metaplasia and eosinophil density in the conducting airways were observed. Adjuvant activity of the $\mathrm{CeO}_{2} \mathrm{NPs}$ was primarily mediated via the Th2 response, while that of the $\mathrm{Co}_{3} \mathrm{O}_{4}$ NPs was characterised by no or less marked increases in IgE plasma levels, BALF IL-4 and IL-5 concentrations and percentages of eosinophils in BALF and more pronounced increases in BALF IL-6 concentrations and percentages of lymphocytes in BALF. Co-exposure to $\mathrm{Co}_{3} \mathrm{O}_{4}$ NPs with OVA and subsequent OVA challenge also induced perivascular and peribronchiolar lymphoid cell accumulation and formation of ectopic lymphoid tissue in lungs. Responses to OVA combined with various NPs were not affected by the amount of doping or redox activity of the NPs.

Conclusions: The findings indicate that chemical composition of NPs influences both the relative potency of NPs to exacerbate allergic airway sensitization and the type of immune response. However, no relation between the acellular redox activity and the observed adjuvant activity of the different NPs was found. Further research is needed to pinpoint the precise physiological properties of NPs and biological mechanisms determining adjuvant activity in order to facilitate a safe-by-design approach to NP development. 


\section{Background}

Engineered nanoparticles (NPs) are under increasing development for a wide-range of applications, however, their potential for toxicity still remains poorly understood. Identifying which physicochemical properties of NPs affect the potential toxicological effects will therefore facilitate predictive risk assessment, grouping and safe-by-design of NPs ${ }^{[1-4]}$.

The relative large surface-to-volume ratio and surface reactivity of NPs are, for example, known to increase reactive oxygen species (ROS) generation ${ }^{[5,6]}$. As a consequence, inhalation of NPs may lead to greater levels of cellular oxidative stress and, subsequently inflammation in the respiratory system, than their larger counterparts. In addition, existing inflammation (e.g. from asthma) can also be exacerbated by NP exposure ${ }^{[7,8]}$. Nano-sized silica and titanium dioxide particles have been shown to enhance allergic airways sensitisation in mice $[9,10]$, however, there is limited understanding of the different physicochemical properties of NPs which influence their adjuvant properties. Previous studies on the influence of NPs on allergic airway sensitisation initially focussed on the role of the dendritic cell and T-cell interaction. Increases in the proliferation, maturation and/or differentiation of dendritic cells and T-cells have been observed after in vitro exposure to NPs and after in vivo exposure to NPs during the sensitisation phase ${ }^{[7]}$. Molecular interactions that drive these cellular responses have been suggested to involve increases in reactive oxygen species (ROS) caused by NPs with an oxidative surface chemistry, inflammasome activation, cellular injury and the induction of dendritic cell simulating cytokines or chemokines by epithelial cells (either direct or via oxidative stress) ${ }^{[7]}$.

Nano-sized metal oxides are known to facilitate the formation of ROS by depleting electrons from cellular redox species (cellular components able to release electrons) or by serving as catalysts in ROS production through Fenton reactions or Haber-Weiss cycle reactions ${ }^{[11]}$. Additionally, NPs can stimulate free radical generation from cellular enzymes. Furthermore, biological fluids (e.g. epithelial lining fluid) and cells also contain antioxidants and numerous compensatory mechanisms, thus the relationship between ROS induction and toxicity is complex.

While there are clear associations between ROS generation and the toxicity of nanomaterials ${ }^{[11-13]}$, the specific role of the redox activity of nanomaterials in the generation of ROS is difficult to investigate, since changing the redox activity of nanomaterials usually also changes other properties (such as the chemical composition or size). In a recent study, we applied chemical doping (intentional substitution of one element by another while maintaining the lattice structure and arrangement) of cerium dioxide to specifically investigate the influence of NP redox activity on ROS formation, and associated induction of oxidative stress responses in mice in vivo ${ }^{[14]}$. Different quantities of zirconium $(\mathrm{Zr})$ were incorporated into the crystalline 
structure of the cerium dioxide nanoparticles $\left(\mathrm{CeO}_{2} \mathrm{NPs}\right)$ to increase the antioxidant potential. However, $\mathrm{Zr}$-doping of $\mathrm{CeO}_{2} \mathrm{NPs}$ had limited effect on the inflammatory responses after inhalation in otherwise healthy mice.

Metal oxides with a conduction band energy (Ec) level that overlaps with the cellular redox potential ( -4.12 to $-4.84 \mathrm{eV}$ ) have been shown to have the ability to induce oxygen radicals, oxidative stress, and acute pulmonary inflammation ${ }^{[15]}$. Therefore, $\mathrm{Co}_{3} \mathrm{O}_{4}$, a metal oxide that (unlike $\mathrm{CeO}_{2}$ ) has an Ec level overlapping with the cellular redox potential was doped using $\mathrm{Fe}_{2} \mathrm{O}_{3}$ or $\mathrm{Fe}_{3} \mathrm{O}_{4}$ ( $\mathrm{Fe}$ oxides themselves do not overlap with the cellular redox potential). Here we present an airway exposure study in a mouse model for airway allergy using the following poorly soluble NPs differing in redox potential but with similar sizes: a) $\mathrm{Co}_{3} \mathrm{O}_{4}$ NPs doped with different amounts of $\mathrm{Fe}, \mathrm{b}$ ) $\mathrm{CeO}_{2}$ NPs doped with different amounts of $\mathrm{Zr}, \mathrm{c}$ ) $\mathrm{CeO}_{2} \mathrm{NM} 212 \mathrm{NPs}$ (for comparison to other in vivo studies) and d) $\mathrm{TiO}_{2} \mathrm{NPs}$ (as a positive control $\left.{ }^{[10]}\right)$. We hypothesise that $\mathrm{Co}_{3} \mathrm{O}_{4} \mathrm{NPs}$ will have a greater ability to enhance allergic airway sensitisation compared to ( $\mathrm{Zr}$-doped) $\mathrm{CeO}_{2} \mathrm{NPs}$, but that these effects of $\mathrm{Co}_{3} \mathrm{O}_{4} \mathrm{NPs}$ can be reduced by Fe-doping. The outcome of this work will facilitate the assessment of the potential hazard of NPs to enhance allergic airway sensitisation and inform efforts to group NPs or to apply a safe-by-design approach in their development.

\section{Methods}

\section{Experimental protocol}

Studies were designed to test the enhancement of allergic sensitization by airway coexposure (intranasal instillation) of an experimental allergen (OVA) with a range of NPs that differ in chemical composition (Figure 1). Animals were divided into 10 test groups and 3 control groups, each with 6 mice except OVA-control $(n=8)$ (see Table 1). On days 1, 3, 6 and 8 the mice were sensitized with $0.02 \%$ OVA in combination with $200 \mu \mathrm{g} \mathrm{TiO}{ }_{2}$ NPs (positive controls), $0.02 \%$ OVA in combination with $200 \mu \mathrm{g}$ of one of the other NPs (test groups), $0.02 \%$ OVA in PBS (OVA control) or PBS alone (PBS control). On days 22 and 23 all mice were challenged with $30 \mu$ of $0.5 \%$ OVA in PBS. The animals were sacrificed 24 hours after the last intranasal challenge (day 24).

A dose of $200 \mu \mathrm{g}$ of NPs was chosen based on our results in previous studies (with doseranges between 100 and $400 \mu \mathrm{g}$ ) using silica ${ }^{[9]}$ and titanium dioxide ${ }^{[10]}$. The mouse model was developed with low concentrations of OVA during sensitization $(0.02 \%)$ that produce minimal allergic responses with challenge, but can be dramatically enhanced with cosensitization with an adjuvant such as particulate matter ${ }^{[9]}$.

No experimental groups were included to measure the effects of exposure to NPs without OVA during the sensitisation phase, since previous studies showed that the allergic 
sensitisation response can only be enhanced via co-exposure of the allergen and $\mathrm{SiO}_{2}$ or $\mathrm{TiO}_{2}$ NPs, and not by exposure to $\mathrm{SiO}_{2}$ and $\mathrm{TiO}_{2}$ NP NPs alone ${ }^{[9,10]}$. Although other studies have shown that separate administration of the allergen and NPs may also lead to adjuvant effects ${ }^{[16]}$, in this study we have chosen to administer OVA and NPs in one suspension, in alignment with our previous studies.

Table 1: Overview of the study groups.

\begin{tabular}{lllll}
\hline group & $\mathbf{n}$ & Day $\mathbf{1}, \mathbf{3}, \mathbf{6}$ and $\mathbf{8}$ & Day 22 and 23 & Day 24 \\
\hline 1 & 6 & PBS controls & OVA & necropsy \\
2 & 8 & OVA controls & OVA & necropsy \\
3 & 6 & $\mathrm{TiO}_{2}+$ OVA (NP positive controls) & OVA & necropsy \\
4 & 6 & $\mathrm{CeO}_{2} \mathrm{NM} 212+$ OVA & OVA & necropsy \\
5 & 6 & $\mathrm{Co}_{3} \mathrm{O}_{4}\left(0 \% \mathrm{Fe}_{2} \mathrm{O}_{3}\right)+$ OVA & OVA & necropsy \\
6 & 6 & $\mathrm{Co}_{3} \mathrm{O}_{4}\left(25 \% \mathrm{Fe}_{2} \mathrm{O}_{3}\right)+$ OVA & OVA & necropsy \\
7 & 6 & $\mathrm{Co}_{3} \mathrm{O}_{4}\left(75 \% \mathrm{Fe}_{2} \mathrm{O}_{3}\right)+$ OVA & OVA & necropsy \\
8 & 6 & $\mathrm{Co}_{3} \mathrm{O}_{4}\left(0 \% \mathrm{Fe}_{3} \mathrm{O}_{4}\right)+$ OVA & OVA & necropsy \\
9 & 6 & $\mathrm{Co}_{3} \mathrm{O}_{4}\left(25 \% \mathrm{Fe}_{3} \mathrm{O}_{4}\right)+$ OVA & OVA & necropsy \\
10 & 6 & $\mathrm{Co}_{3} \mathrm{O}_{4}\left(75 \% \mathrm{Fe}_{3} \mathrm{O}_{4}\right)+$ OVA & OVA & necropsy \\
11 & 6 & $\mathrm{CeO}_{2}(0 \% \mathrm{Zr})+$ OVA & OVA & necropsy \\
12 & 6 & $\mathrm{CeO}_{2}(27 \% \mathrm{Zr})+$ OVA & OVA & necropsy \\
13 & 6 & $\mathrm{CeO}_{2}(78 \% \mathrm{Zr})+$ OVA & OVA & necropsy \\
\hline
\end{tabular}

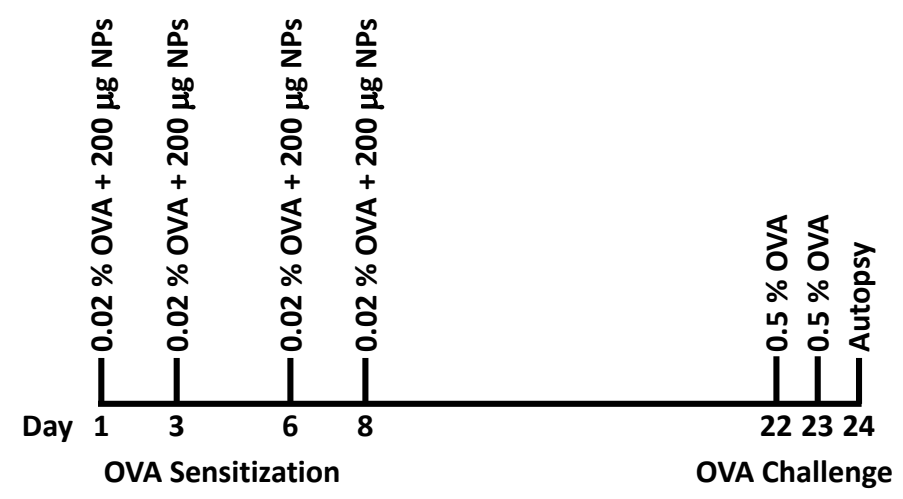

Figure 1: Schematic overview of the study design. 


\section{Nanomaterial production and characterization}

$\mathrm{TiO}_{2}$ NPs (NO-0058-HP) were obtained from lonic Liquids Technologies GmbH, Germany. These uncoated anatase $\mathrm{TiO}_{2}$ NPs were the same NPs as used on our previous in vivo study ${ }^{[10]}$. The OECD representative manufactured nanomaterial $\mathrm{CeO}_{2}(\mathrm{NM} 212)$ was obtained from Umicore, Belgium. The other $\mathrm{CeO}_{2} \mathrm{NPs}, \mathrm{Zr}$-doped $\mathrm{CeO}_{2} \mathrm{NPs}, \mathrm{Co}_{3} \mathrm{O}_{4} \mathrm{NPs}$ and Fe-doped $\mathrm{Co}_{3} \mathrm{O}_{4}$ NPs were produced using supercritical water hydrothermal synthesis ${ }^{[17,18]}$. Briefly, $\mathrm{H}_{2} \mathrm{O}$ was pumped through a pre-heating coil $\left(\sim 400^{\circ} \mathrm{C}\right)$, brought into contact with a concurrently flowing solution of metal salts at room temperature (RT), while maintaining the flow rates, temperature and the pressure constant at 240 bar. The mixture was cooled immediately after the mixing point and passed through a back pressure regulator to decrease the pressure back down to ambient conditions. Solids in the aqueous suspensions were washed by centrifuging and re-dispersing in clean MilliQ water 3 times. The particles were characterised in suspension or, where solid particles were required, the suspensions were oven dried $\left(\sim 100^{\circ} \mathrm{C}\right)$. For the synthesis of the Fe-doped $\mathrm{Co}_{3} \mathrm{O}_{4} \mathrm{NPs}$, different ratios of cobalt and iron salts were used as precursors. Two different iron salts (iron(III) nitrate nonahydrate and ammonium iron(III) citrate) were used, resulting in two different series of NPs, with $\mathrm{Fe}_{2} \mathrm{O}_{3}$ or $\mathrm{Fe}_{3} \mathrm{O}_{4}$ as a precursor, respectively. These different series $\left(\mathrm{Co}_{3} \mathrm{O}_{4}\left(x \% \mathrm{Fe}_{2} \mathrm{O}_{3}\right) \mathrm{NPs}\right.$ and $\mathrm{Co}_{3} \mathrm{O}_{4}\left(\mathrm{x} \% \mathrm{Fe}_{3} \mathrm{O}_{4}\right) \mathrm{NPs}$ ) have different degrees of crystallinity, aggregation, Co/Fe ratio and spatial distributions of the metals in the NPs ${ }^{[18]}$.

The particle size in ultra-pure water was measured with disc centrifuge. The particle size in cell culture medium (RPMI 1640, Gibco) with 25mM Hepes and 2mM L-Glutamine, $1 \%$ Penicilline-Streptomycine (Pen/Strep $10000 \mathrm{U} / \mathrm{mL}$, Glibco) and 10\% heat inactivated Fetal Calf Serum (FCS, Glibco), was measured with dynamic light scattering (DLS). The redox activity was tested with electron paramagnetic resonance (EPR) using a cell free system with a superoxide-selective spin-trap (tempone-H) to detect the generation of superoxide production and the spin trap DMPO ${ }^{[19]}$ in combination with $\mathrm{H}_{2} \mathrm{O}_{2}$ and $\mathrm{CuSO}_{4}$ to analyse the capacity to scavenge hydroxyl free radicals. For the superoxide-selective spin-trap nanoparticle suspensions $(0.56 \mathrm{mg} / \mathrm{mL})$ were prepared and incubated with the spin-trap, Tempone- $\mathrm{H}(1 \mathrm{mM})$ immediately before the initial measurement. Samples were kept at $37^{\circ} \mathrm{C}$ throughout and measurements were taken with an X-band EPR spectrometer (Magnettech MS-200, Berlin, Germany) at $60 \mathrm{~min}$ by drawing $50 \mu \mathrm{L}$ of sample into a capillary tube (Scientific Laboratory Ltd, Coatbridge, UK) and sealing with a plug of soft sealant (Cristaseal, VWR International, UK). For the DMPO spin trap a sample was made of $12.5 \mu \mathrm{L}$ nanoparticle suspension $(1.28 \mathrm{mg} / \mathrm{mL}), 12.5 \mu \mathrm{LCuSO}_{4}(20 \mu \mathrm{M}), 25 \mu \mathrm{L} \mathrm{H}_{2} \mathrm{O}_{2}(0.5 \mathrm{M})$ and $50 \mu \mathrm{L}$ DMPO $(0.05 \mathrm{M})$. This sample was incubated in a shaker water bath at $37^{\circ} \mathrm{C}$ for 15 minutes at 100 rpm, vortexed and taken up in a capillary tube, which was then sealed at the bottom with haematocrit and measured with the ESR Spectroscope (Miniscope MS 400; MT MagnetTech $\mathrm{GmbH}$ ). All NPs were tested for endotoxin contamination using either a Limulus Amoebocyte Lysate Assay (chromogenic kinetic LAL assay) or LC-MS/MS determination ${ }^{[20]}$. 


\section{Animals}

Female BALB/c mice ( 20 grams body weight; 6-8 weeks old) were obtained from Charles River (Portage, MI). Mice were maintained at the Michigan State University (MSU) animal housing facilities at room temperature $\left(21^{\circ} \mathrm{C}-24^{\circ} \mathrm{C}\right)$ and relative humidity of $45-70 \%$, with a $12 \mathrm{~h}$ light/dark cycle. MSU is AAALAC accredited and all animal procedures \& experimental protocols were approved by the MSU Institutional Animal Care and Use Committee.

\section{Intranasal Instillation}

Mice were lightly anesthetized for 1-2 min with 4\% isoflurane in oxygen using an isoflurane vaporizer. Cradling the mouse in an upright position, the thumb was used to support the lower jaw and position the nose of the mouse so it was easily accessible. A volume of $30 \mu \mathrm{L}$ was delivered to the tip of the nares. Once the instillate was fully inhaled, the mouse was placed back in its cage and monitored until conscious with normal respirations.

\section{Necropsy, lavage collection and tissue preparation}

Mice were anesthetized with an intraperitoneal injection of sodium pentobarbital $(60 \mathrm{mg} / \mathrm{kg}$ body weight). A midline laparotomy was performed and approximately $0.5 \mathrm{~mL}$ of blood was drawn from the vena cava and collected in heparinized tubes (BD Microtainer, Franklin Lakes, $\mathrm{NJ}$ ) for separation of plasma. The plasma was stored at $-80^{\circ} \mathrm{C}$ for later biochemical analysis (OVA-specific IgE and IgG1). Animals were exsanguinated and the trachea was exposed and cannulated, and the heart and lungs were excised en bloc. A volume of $0.8 \mathrm{~mL}$ sterile saline was instilled through the tracheal cannula and withdrawn to recover bronchoalveolar lavage fluid (BALF). A second intratracheal saline lavage was performed and the collected BALF was combined with the first sample for analysis.

After the BALF was collected, the left lung lobe was intratracheally fixed with neutral-buffered formalin at a constant pressure $\left(30 \mathrm{~cm} \mathrm{H}_{2} \mathrm{O}\right)$ for $2 \mathrm{~h}$ and then submersed in the same fixative for $>24 \mathrm{~h}$ until further tissue processing for light microscopy. Two sections were excised at the level of the $5^{\text {th }}$ and $11^{\text {th }}$ airway along the main axial airway (G5 and G11), to sample proximal and distal bronchiolar airways, respectively ${ }^{[21]}$. Tissue was embedded in paraffin and 5 - to $6-\mu \mathrm{m}$-thick sections were cut from the anterior surface. Lung sections were stained with hematoxylin and eosin (H\&E) for routine light microscopic examination and with Alcian Blue ( $\mathrm{pH}$ 2.5)/Periodic Acid-Schiff (AB/PAS) for identification of intraepithelial neutral and acidic mucosubstances in pulmonary bronchiolar epithelium. To detect eosinophils, slides were immunostained using a polyclonal rabbit antibody directed against murine eosinophilspecific major basic protein (MBP; 1:500; Mayo Clinic, AZ). Accumulations of B-lymphocytes in lung tissues were detected by immunohistochemical staining with rat anti-CD45R monoclonal (1:600; Becton Dickinson, Franklin Lakes, NJ, catalog \# 550286). 


\section{OVA-specific IgE and IgG1 in plasma}

Plasma was separated from blood and analysed for OVA-specific IgE and IgG1 using an ELISA kit (Cayman, Ann Arbor, MI) according to the manufacturer's instructions. The plasma samples were diluted 8 times for the IgE and 1000 times for the $\lg \mathrm{G} 1$ analysis.

\section{BALF analyses}

The total number of cells in the BALF was determined using a hemocytometer. Cytological slides were prepared by centrifugation at $40 \mathrm{xg}$ for $10 \mathrm{~min}$ at $20^{\circ} \mathrm{C}$ using a Shandon cytospin 3 (Shandon Scientific, PA) and stained with Diff-Quick (Dade Behring, DE). Differential cell counts for neutrophils, eosinophils, macrophages/monocytes, and lymphocytes were assessed from a total of at least 200 cells. The remaining BALF was centrifuged at $240 \mathrm{xg}$ for $15 \mathrm{~min}$ at $4^{\circ} \mathrm{C}$ and the supernatant was collected and stored at $-80^{\circ} \mathrm{C}$ for subsequent measurement of inflammatory cytokines (IFNy, IL-4, IL-5, IL-13, IL-6, IL-13, IL-17 and TNF $\alpha$ ) using a Luminex-kit (Millipore, MA).

\section{Lung morphometry}

Histologic slides with lung tissue sections were scanned and digitized with a slide scanner (VS110, Olympus America, Center Valley, PA), and evaluated using stereological methods with newCAST software (VisioPharm, Hoersholm, Denmark). For quantification of major basic protein (MBP)-positive eosinophils, digitized images of the lung were selected as regions of interest and $40 \%$ of the lung tissue was captured at $400 x$ magnification by systematic random sampling. Percentage of MBP-positive cells in the total lung tissue and in three discrete regions of 1 ) the parenchyma (alveoli and alveolar ducts), 2) perivascular and peribronchial interstitial spaces, and 3 ) other regions (inside blood vessels, bronchiole airspaces and pleura), were estimated using Stepanizer stereology software with a point grid by dividing the number of points hitting areas positive for MPB $\left(a_{(p) \text { positive }}\right)$ by the total number of points falling on all lung tissue (MBP-positive and-negative; $a_{(p) \text { reference tissue }}$ ). For each region, percent density of eosinophils was calculated with the equation:

$$
\text { Eosinophil Density }(\%)=\frac{(\text { No.positive cells }) \times a_{(p) \text { positive cell }}}{(\text { No.reference tissue }) \times a_{(p) \text { reference tissue }}} \times 100
$$

For quantification of CD45R-positive staining cells (the B-lymphocyte density), digitized images of G5 and G11 lung sections were selected as regions of interest and $40 \%$ of the lung tissue was captured at $400 x$ magnification by systematic random sampling. Percentage of CD45R-positive cells in the perivascular and peribronchial interstitial spaces were estimated using Stepanizer stereology software using a point grid by dividing the number of points hitting areas positive for CD45R ( $a_{(p) \text { positive }}$ ) by the total number of points falling on all lung tissue (CD45R-positive and-negative; $\left.a_{(p) \text { reference tissue }}\right)$. For each region, percent density of B-lymphocytes was calculated with the equation: 
$B-$ lymphocyte Density $(\%)=\frac{(\text { No.positive cells }) \times a_{(p) \text { positive cell }}}{(\text { No.reference tissue }) \times a_{(p) \text { reference tissue }}} \times 100$

For quantification of $A B / P A S-p o s i t i v e ~ m u c o s u b s t a n c e s$ in the bronchiolar epithelium, all bronchiolar epithelium lining the main axial airway was selected and captured at $400 x$ magnification. A point intercept grid was placed over the sampled images to estimate the density of mucosubstances per basal lamina. The number of points hitting AB/PAS-positive mucosubstances $\left(P_{m}\right)$ was counted. The density of AB/PAS-positive mucosubstances $\left(\hat{V}_{m}\right)$ was estimated by multiplying the total number of $P_{m}$ by the area/point $(a / p)$ and dividing them by the number of points hitting the reference space $(n)$ as shown in the equation.

$\hat{V}_{m}=\frac{\sum P_{m} \times a / p}{n}$

The surface density of the basal lamina $\left(\hat{S}_{\mathrm{BL}}\right)$ in the selected images was estimated by counting the number of intercepts $(I)$ of the line probe with the basal lamina of the lateral wall divided by the length per point $(I / p)$ and the number of points falling on the reference space $(n)$ as described:

$\hat{S}_{B L}=\frac{2 \times \sum I}{l / p \times n}$

The positive density per basal lamina of the bronchiolar epithelium was then estimated by dividing $\hat{\mathrm{V}}_{\mathrm{m}}$ by $\hat{\mathrm{S}}_{\mathrm{BL}}$.

\section{Statistical analyses}

GraphPad Prism v7.00 (GraphPad Software, San Diego, California, USA) was used to analyse the data. All data are depicted as group means \pm standard deviation (SD). First, outliers were identified with Grubbs' test (alpha $=0.05$ ) and removed. The Shapiro-Wilk test and the Brown-Forsythe test were used to test for normality and equal variances, respectively. Differences between the OVA control group and a) the other control groups (PBS or $\mathrm{TiO}_{2}$ NP exposed), b) the $\mathrm{CeO}_{2}$ NP exposed groups, c) the $\mathrm{Co}_{3} \mathrm{O}_{4}\left(\mathrm{x} \% \mathrm{Fe}_{2} \mathrm{O}_{3}\right)$ NP exposed groups and d) the $\mathrm{Co}_{3} \mathrm{O}_{4}\left(x \% \mathrm{Fe}_{3} \mathrm{O}_{4}\right)$ NPs exposed groups were analysed using a one-way analysis of variance (ANOVA), followed by a Dunnett's post-hoc multiple comparisons test comparing groups exposed to OVA with NPs to groups exposed to OVA alone. For all statistical analyses, a $p$-value of $\leq 0.05$ was considered statistically significant.

If the $p$-value of the Shapiro-Wilk or Brown-Forsythe test was $\leq 0.05$, the data were lognormally transformed and again tested for normality and equal variances. If the $p$-values of the Shapiro-Wilk and Brown-Forsythe tests of the log-normally transformed data were $>0.05$, a one-way ANOVA followed by a Dunnett's post-hoc multiple comparisons test was performed on the log-normally transformed data. If the $p$-values of the Shapiro-Wilk test 
were still $\leq 0.05$, a non-parametric (Kruskal Wallis) ANOVA was performed on the nontransformed data followed by a Dunn's post-hoc multiple comparisons test.

\section{Results}

\section{Nanomaterial characterization}

Fe-doping had small, but not statistically significant, effects on NP size in suspension (Table 2). Other characteristics, such as shape and crystalline structure, did not significantly change with increasing amounts of doping. In Figure 2, the redox activity of the NPs as measured in a cell free system by EPR using two different spin-traps are shown. EPR analysis using tempone- $\mathrm{H}$ demonstrated an increased capacity of $\mathrm{Co}_{3} \mathrm{O}_{4}\left(25 \% \mathrm{Fe}_{3} \mathrm{O}_{4}\right)$ NPs to generate reactive superoxide free radicals compared to $\mathrm{Co}_{3} \mathrm{O}_{4}\left(0\right.$ and $\left.75 \% \mathrm{Fe}_{3} \mathrm{O}_{4}\right)$ NPs. Increasing amounts of Fe-doping using $\mathrm{Fe}_{3} \mathrm{O}_{4}$ led a significant decrease in scavenging capacity of $\mathrm{Co}_{3} \mathrm{O}_{4}$ NPs. $\mathrm{Co}_{3} \mathrm{O}_{4}\left(0,25\right.$ and $\left.75 \% \mathrm{Fe}_{2} \mathrm{O}_{3}\right)$ NPs had a similar ROS generation and scavenging capacity (Figure 2). Endotoxin levels were below the recommended endotoxin limits for preclinical research in animal models ( $5 \mathrm{EU} / \mathrm{kg}$ bw/day or $36 \mathrm{EU} / \mathrm{mL}$ for mice with a body weight of $30 \mathrm{~g}$ and a daily dose of $100 \mu \mathrm{L}$ ) ${ }^{[22]}$.

Table 2: Physicochemical characteristics of the nanoparticles.

\begin{tabular}{|c|c|c|c|}
\hline & $\begin{array}{l}\text { Primary particle size } \\
\text { (Mean } \pm \text { SD) }(\mathrm{nm})\end{array}$ & $\begin{array}{l}\text { Particle diameter in water } \\
\text { (Median) (nm) }\end{array}$ & $\begin{array}{l}\text { Particle diameter in cell culture } \\
\text { medium }+10 \% \text { foetal calf serum } \\
\text { (Mean } \pm \text { SD) (arbitrary units) }\end{array}$ \\
\hline Measured with $\rightarrow$ & STEM & Disc Centrifuge (128 $\mu \mathrm{g} / \mathrm{mL})$ & DLS (128 $\mu \mathrm{g} / \mathrm{mL})$ \\
\hline Anatase $\mathrm{TiO}_{2}$ & $10-15^{\mathrm{a}}$ & $121^{\mathrm{b}}$ & - \\
\hline $\mathrm{CeO}_{2} \mathrm{NM} 212$ & $17.1 \pm 10.9$ & - & $428 \pm 11.7$ \\
\hline $\mathrm{CeO}_{2}(0 \% \mathrm{Zr})$ & $4.7 \pm 1.4$ & 39 & $288 \pm 4.8$ \\
\hline $\mathrm{CeO}_{2}(27 \% \mathrm{Zr})$ & $4.6 \pm 1.4$ & 40 & $176 \pm 4.0$ \\
\hline $\mathrm{CeO}_{2}(78 \% \mathrm{Zr})$ & $4.7 \pm 1.4$ & 41 & $415 \pm 27.6$ \\
\hline $\mathrm{Co}_{3} \mathrm{O}_{4}\left(0 \% \mathrm{Fe}_{2} \mathrm{O}_{3}\right)$ & $17.5 \pm 15.0$ & 42 & $176 \pm 2.9$ \\
\hline $\mathrm{Co}_{3} \mathrm{O}_{4}\left(25 \% \mathrm{Fe}_{2} \mathrm{O}_{3}\right)$ & $10.6 \pm 3.6$ & 62 & $303 \pm 6.7$ \\
\hline $\mathrm{Co}_{3} \mathrm{O}_{4}\left(75 \% \mathrm{Fe}_{2} \mathrm{O}_{3}\right)$ & $8.6 \pm 1.7$ & 39 & $373 \pm 9.9$ \\
\hline $\mathrm{Co}_{3} \mathrm{O}_{4}\left(0 \% \mathrm{Fe}_{3} \mathrm{O}_{4}\right)$ & $18.7 \pm 11.2$ & 44 & $160 \pm 1.3$ \\
\hline $\mathrm{Co}_{3} \mathrm{O}_{4}\left(25 \% \mathrm{Fe}_{3} \mathrm{O}_{4}\right)$ & $13.0 \pm 5.2$ & 61 & $828 \pm 3.8$ \\
\hline $\mathrm{Co}_{3} \mathrm{O}_{4}\left(75 \% \mathrm{Fe}_{3} \mathrm{O}_{4}\right)$ & $10.2 \pm 6.2$ & 69 & $1165 \pm 66.5$ \\
\hline
\end{tabular}

a Information provided by the manufacturer instead of measured with STEM;

b Particles size as measured in our previous study with Nanoparticle Tracking Analysis instead of Disc Centrifuge; $\mathrm{SD}=$ Standard Deviation, STEM = Scanning Transmission Electron Microscopy; DLS = Dynamic Light Scattering, - = no data available. 
a

EPR measurements of the superoxide generation of several ENMs

$\mathrm{CeO}_{2} \quad \mathrm{CO}_{3} \mathrm{O}_{4} \quad \mathrm{CO}_{3} \mathrm{O}_{4}$

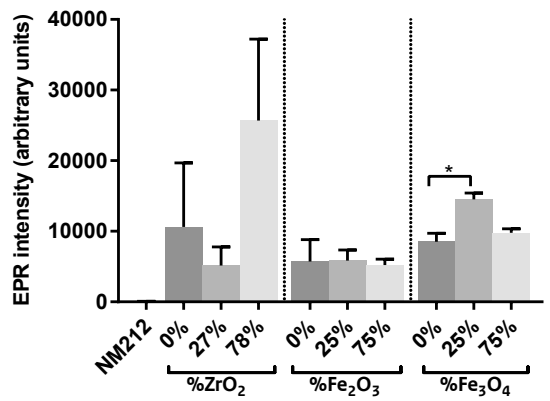

b EPR measurements of the
scavenging capacity of several ENMs

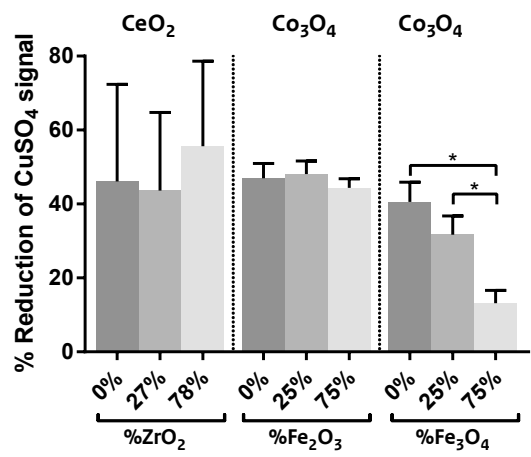

Figure 2: Reactive oxygen species (ROS) generation and scavenging capacity of NPs.

Superoxide generation of NPs measured in a cell free system by electron paramagnetic resonance (EPR) using Tempone-H (a). The EPR signal of the $\mathrm{Co}_{3} \mathrm{O}_{4}\left(25 \% \mathrm{Fe}_{3} \mathrm{O}_{4}\right)$ NPs was statistically significantly higher than the $\mathrm{Co}_{3} \mathrm{O}_{4}(0 \%$ $\left.\mathrm{Fe}_{3} \mathrm{O}_{4}\right)$ NPs $(n=4)$ indicating a larger capacity to generate ROS. Scavenging capacity of several NPs expressed as the

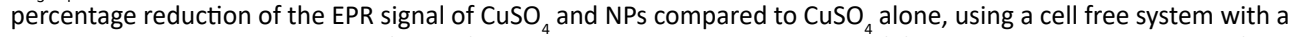
5,5-dimethyl-1-pyrroline $\mathrm{N}$-oxide (DMPO) spin trap in combination with $\mathrm{H}_{2} \mathrm{O}_{2}$ (b). The percentage reduction of the $\mathrm{CuSO}_{4}$ signal by the $\mathrm{Co}_{3} \mathrm{O}_{4}\left(75 \% \mathrm{Fe}_{3} \mathrm{O}_{4}\right) \mathrm{NPs}$ was significantly lower than that ofthe $\mathrm{Co}_{3} \mathrm{O}_{4}\left(0\right.$ and $\left.25 \% \mathrm{Fe}_{3} \mathrm{O}_{4}\right) \mathrm{NPs}(\mathrm{n}=3)$, indicating a lower scavenging capacity of ROS.

\section{OVA-specific IgE and IgG1 in plasma}

OVA-specific IgE and IgG1 antibodies in plasma, indicating an OVA-specific immune response, were measured using an ELISA kit. OVA sensitization (0.02\% OVA) and challenge (0.5\% OVA) caused minimal, non-significant increases in plasma OVA-specific IgE and IgG1 compared to non-sensitized mice (phosphate-buffered saline (PBS) treated controls). Co-sensitization with NPs further increased the plasma OVA-specific IgE or IgG1 concentrations for all NPs. For all NPs, except for $\mathrm{Co}_{3} \mathrm{O}_{4}\left(0\right.$ and $\left.75 \% \mathrm{Fe}_{3} \mathrm{O}_{4}\right)$ NPs, the OVA-specific IgE and/or the IgG1 concentration was statistically significantly increased compared to OVA alone (Figure 3).

\section{Plasma OVA-specific IgE}

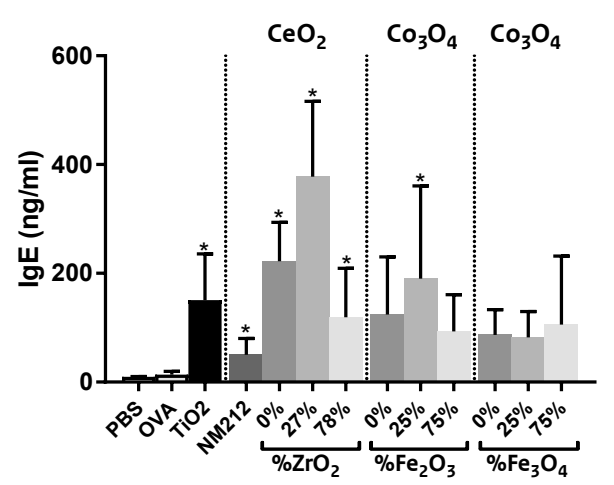

Plasma OVA-specific IgG1

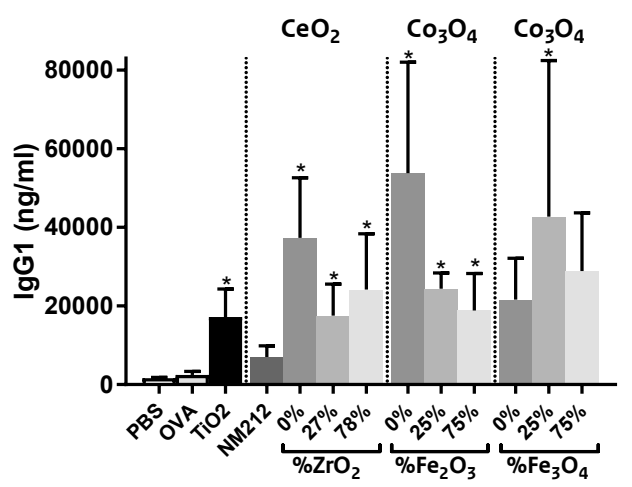

Figure 3: Concentration of OVA-specific IgE and IgG1 in plasma.

Mean $\pm S D, n=6$ except OVA controls where $n=8,{ }^{*}=$ statistically significant different from OVA controls $(p<0.05)$. 


\section{BALF analyses}

\section{BALF total cell count}

The total and differential cell counts were determined using a hemocytometer and analysis of cytospin prepared slides. All mice sensitized with NP plus OVA showed a significant increase in total BALF cells compared to the OVA controls, indicating an increased inflammatory response, except for animals exposed to $\mathrm{Co}_{3} \mathrm{O}_{4}\left(0 \% \mathrm{Fe}_{2} \mathrm{O}_{3}\right) \mathrm{NPs}(\mathrm{p}=0.18$; see Figure 4a). For $\mathrm{CeO}_{2}$ NPs the total cell count increased with increasing amounts of $\mathrm{Zr}$ doping.

\section{Neutrophils}

An increase in neutrophils in the BALF is indicative for a non-allergic, acute inflammatory response. No statistically significant differences between the percentage of neutrophils of the OVA controls and that of OVA plus NP exposed animals were observed, except for a decreased percentage of neutrophils in mice exposed to OVA plus $\mathrm{CeO}_{2}(78 \% \mathrm{Zr}$ ) NPs, $\mathrm{Co}_{3} \mathrm{O}_{4}\left(25\right.$ and $\left.75 \% \mathrm{Fe}_{2} \mathrm{O}_{3}\right)$ NPs and $\mathrm{Co}_{3} \mathrm{O}_{4}\left(75 \% \mathrm{Fe}_{3} \mathrm{O}_{4}\right)$ NPs. No major differences were observed between the different types of NPs (see Figure $4 \mathrm{~b}$ ). The percentage of neutrophils decreased with the amount of doping for $\mathrm{Zr}$-doped $\mathrm{CeO}_{2} \mathrm{NPs}$ and Fe-doped $\mathrm{Co}_{3} \mathrm{O}_{4} \mathrm{NPs}$ (using both $\mathrm{Fe}_{2} \mathrm{O}_{3}$ and $\mathrm{Fe}_{3} \mathrm{O}_{4}$ ).

\section{Eosinophils and lymphocytes}

The animals exposed to OVA plus $\mathrm{CeO}_{2} \mathrm{NPs}$ showed a statistically significant increase in the percentage of eosinophils compared to the OVA controls (Figure 4c), which is a typical feature of allergic asthma. For $\mathrm{CeO}_{2}(27$ and $78 \% \mathrm{Zr}$ ) NPs there was also a statistically significant increase in the percentage of lymphocytes (Figure $4 \mathrm{~d}$ ), indicative for a chronic inflammatory response. Fe-doped $\mathrm{Co}_{3} \mathrm{O}_{4} \mathrm{NPs}$ (using both $\mathrm{Fe}_{2} \mathrm{O}_{3}$ and $\mathrm{Fe}_{3} \mathrm{O}_{4}$ ) caused a statistically significant increase in the percentage of lymphocytes only, and not of eosinophils.

\section{Monocytes}

No major differences in the percentage of monocytes were observed between mice sensitized with NP plus OVA compared to the OVA controls (data not shown). More detailed data, including the absolute differential cell counts, can be found in Additional file 1. 

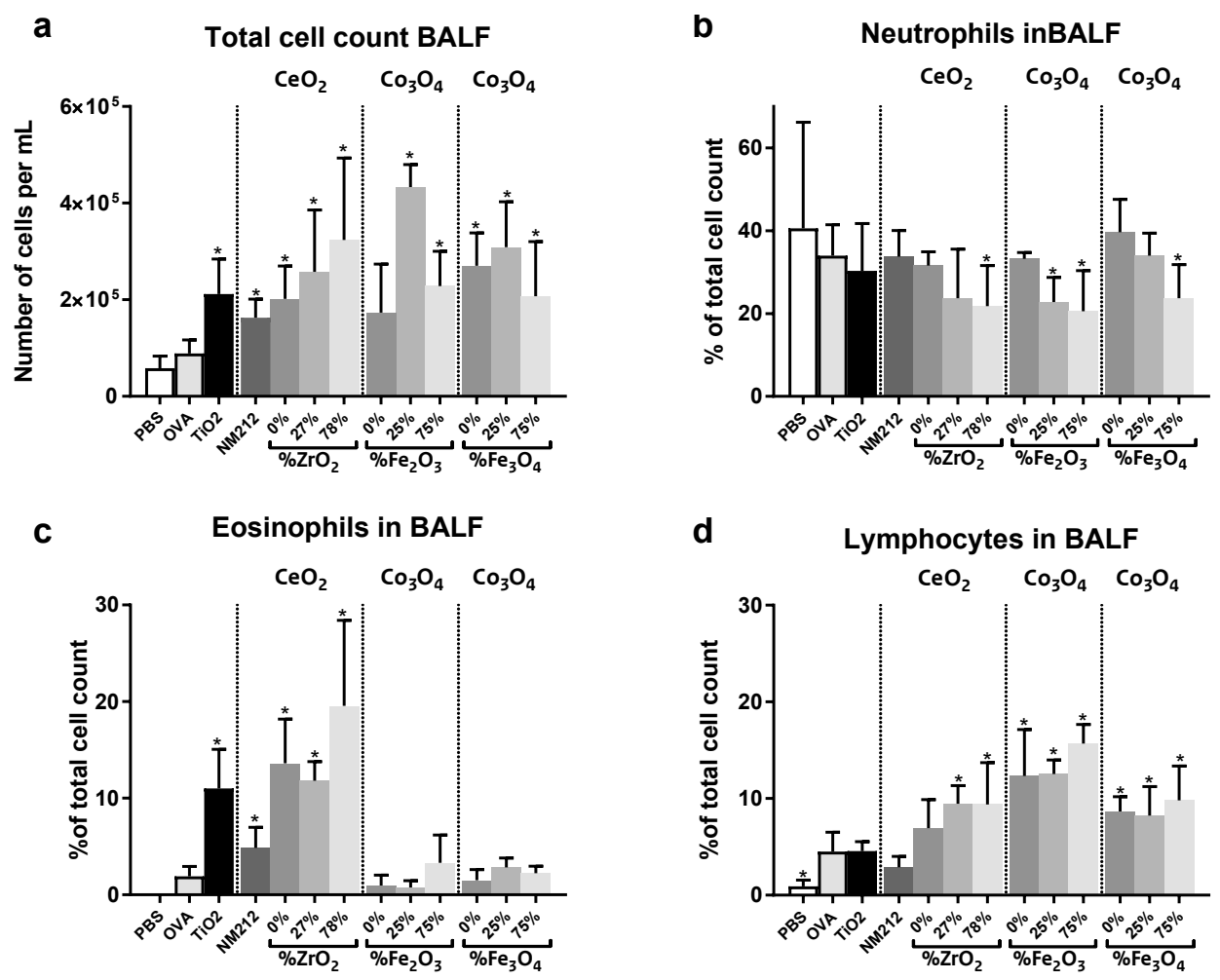

Figure 4: Differential cell counts in bronchoalveolar lavage fluid (BALF).

Total cell count (a) and percentage of neutrophils (b), eosinophils (c) and lymphocytes (d) in the BALF. Mean \pm SD, $n=6$ except OVA where $n=8,{ }^{*}=$ statistically significant different from OVA controls $(p<0.05)$.

\section{BALF cytokines}

The concentrations of several inflammatory cytokines in the BALF were determined in using a Luminex-kit specific for the various cytokines evaluated. Animals exposed to OVA plus $\mathrm{CeO}_{2}$ NPs showed increased concentrations of the Th2 cytokines IL-4 and IL-5 (but not IL-6 or IL-13) in the BALF compared to the OVA controls. Animals exposed to OVA plus most undoped and Fe-doped $\mathrm{Co}_{3} \mathrm{O}_{4}$ NPs showed increased concentrations of IL- 6 compared to the OVA controls. All animals, including the controls (exposed to PBS, OVA and OVA plus $\mathrm{TiO}_{2}$ NPs during sensitization) had detectable concentrations of IL-13 $(20-50 \mathrm{ng} / \mathrm{mL})$ but these were not significantly different from one another (see Figure $5 \mathrm{~d}$ ).

The concentrations of the other cytokines (IFNy, IL-1 $\beta$, IL-17 and TNF $\alpha$ ) measured in BALF were either below the limits of detection or showed no significant changes in the OVA plus NP exposed groups compared to the OVA controls. More detailed data can be found in Additional file 2. 

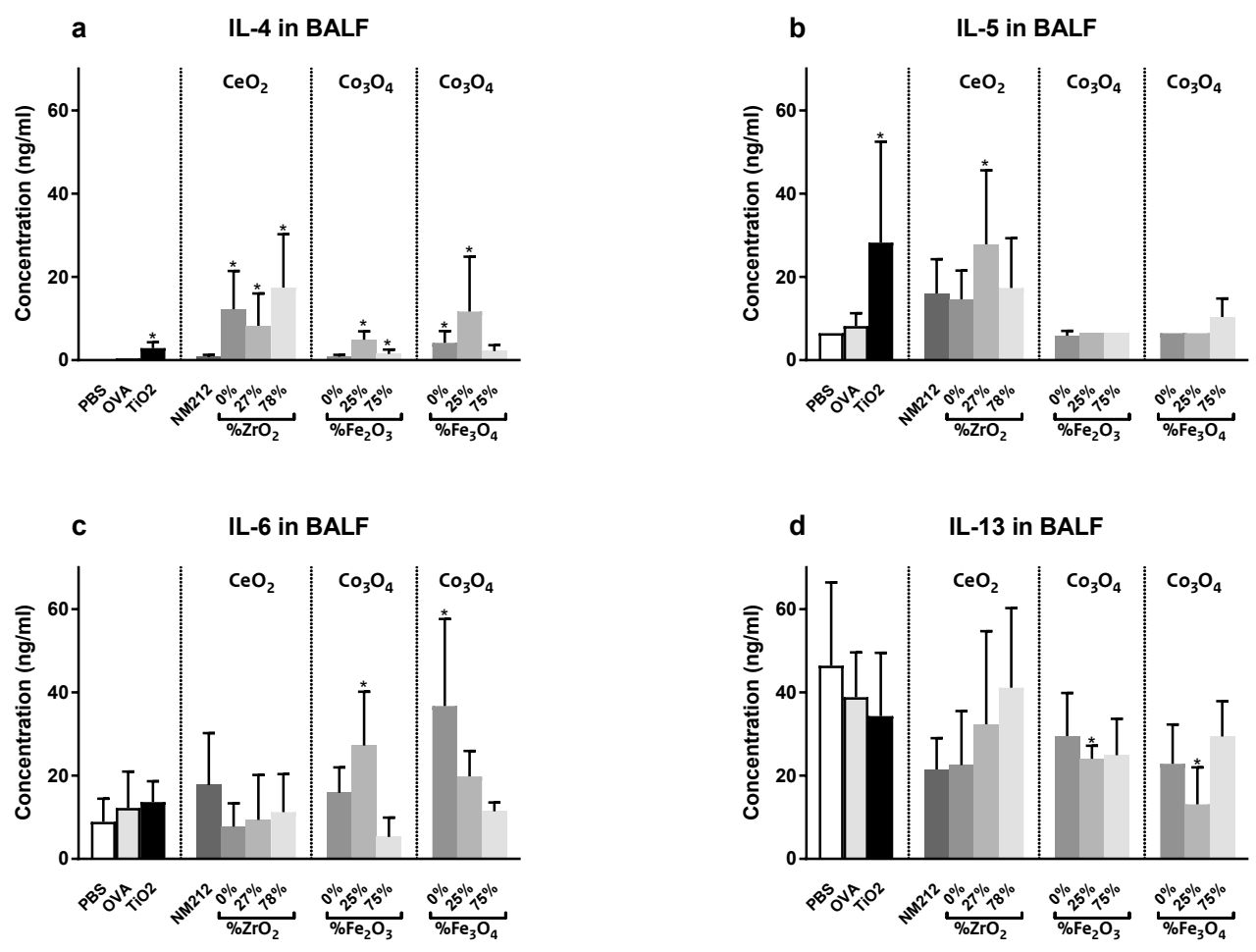

Figure 5: Cytokines in bronchoalveolar lavage fluid (BALF).

Concentration of IL-4 (a), IL-5 (b), IL-6 (c) and IL-13 (d), in the BALF. Mean \pm SD, $n=6$ except OVA where $n=8$, $*=$ statistically significant different from OVA controls $(p<0.05)$.

\section{Lung morphology}

\section{Mucous cell metaplasia.}

For quantification of epithelial mucous, lung tissue sections were stained with Alcian Blue/ Periodic Acid Schiff (AB/PAS), a marker for neutral and acidic mucosubstances. Increased epithelial mucous production is a characteristic feature of allergic airway disease.

Sensitization and challenge with OVA caused a significant accumulation of intraepithelial mucosubstances (mucous cell metaplasia) in the epithelium lining the main proximal axial airways (G5), which was further enhanced by co-administration of OVA with $\mathrm{TiO}_{2} \mathrm{NPS}$ (positive control). All $\mathrm{CeO}_{2}$ NPs enhanced mucosubstance accumulation; this was statistically significant for $\mathrm{CeO}_{2} \mathrm{NM} 212$ and $\mathrm{CeO}_{2}$ with $27 \%$, but not 78\%, $\mathrm{Zr}$ doping (see Figure 6).

By comparison, in the distal axial airways (G11), OVA sensitization and challenge did not affect epithelial accumulated mucosubstances. Co-sensitization with $\mathrm{OVA}_{\text {plus }} \mathrm{TiO}_{2}$ (positive control), $\mathrm{CeO}_{2} \mathrm{NM} 212, \mathrm{CeO}_{2}(0,27$ and $78 \% \mathrm{Zr}), \mathrm{Co}_{3} \mathrm{O}_{4}\left(25 \% \mathrm{Fe}_{2} \mathrm{O}_{3}\right)$ and $\mathrm{Co}_{3} \mathrm{O}_{4}\left(25 \% \mathrm{Fe}_{3} \mathrm{O}_{4}\right) \mathrm{NPs}$ led to a statistically significant increase in mucosubstances compared to the OVA controls. 

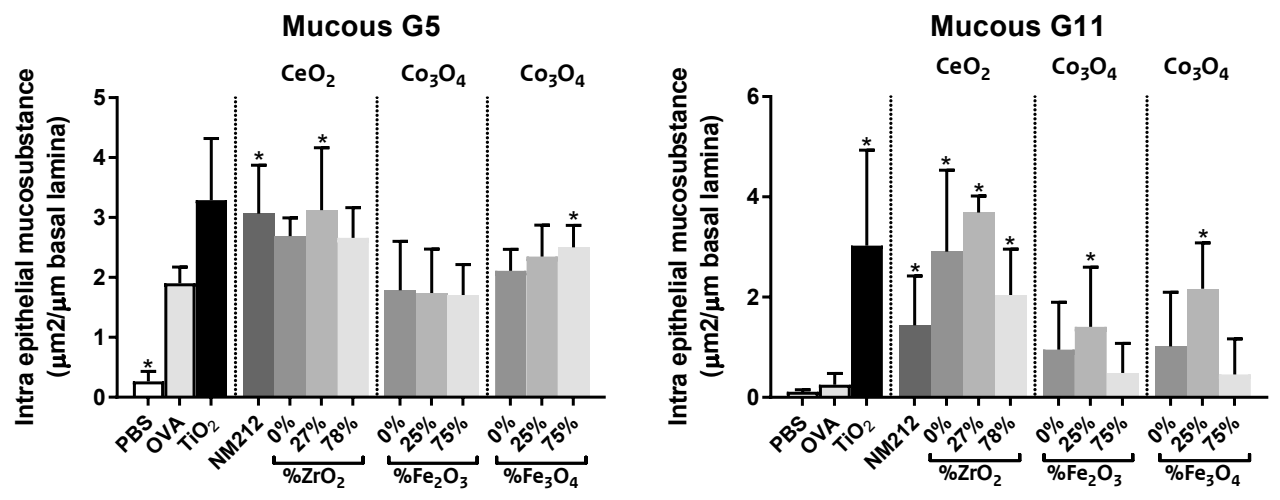

Figure 6: Mucous cell metaplasia.

Quantification of the increase in intraepithelial mucosubstance in the epithelium lining the main proximal axial airway (G5;left) and distal axial airway (G11;right) of the left lung lobe. Mean $\pm S D, n=6$ except OVA where $n=8$, $*=$ statistically significant different from OVA controls $(p<0.05)$.

\section{Eosinophil density}

For quantification of the eosinophil density, a biomarker for an allergic airway response, lung tissue sections were stained for murine major basic protein (MBP), a specific marker for eosinophils.

Parenchymal lung tissue - OVA sensitization and challenge did not increase the overall eosinophil density in the pulmonary parenchyma compared to non-sensitized mice (PBS controls). Co-sensitization of OVA with $\mathrm{TiO}_{2}$ (positive control) and $\mathrm{CeO}_{2} \mathrm{NPs}$ significantly increased eosinophil density compared to OVA alone. This increase was statistically significant in all these groups, except for the OVA plus $\mathrm{CeO}_{2} \mathrm{NM} 212$ NPs group. Co-sensitization of OVA with undoped and doped $\mathrm{Co}_{3} \mathrm{O}_{4}$ NPs had no effect on eosinophil density (Figure 7a).

Perivascular and peribronchial lung tissue - OVA sensitization and challenge did not significantly affect the eosinophil density in the peribronchial and perivascular region of the lung compared to non-sensitized mice (PBS controls). Co-sensitization of OVA with $\mathrm{TiO}_{2}$ (positive control), $\mathrm{CeO}_{2}\left(0,27\right.$ and $78 \% \mathrm{Zr}$ ) and $\mathrm{CO}_{3} \mathrm{O}_{4}\left(25\right.$ and $\left.75 \% \mathrm{Fe}_{3} \mathrm{O}_{4}\right)$ NPs caused significant increases in eosinophil density compared to OVA alone (Figure $7 \mathrm{~b}$ ).

Total lung - OVA sensitization and challenge did not significantly alter the overall pulmonary eosinophil density compared to non-sensitized mice (PBS controls). Co-sensitization of OVA with $\mathrm{TiO}_{2} \mathrm{NPs}$ (positive control), $\mathrm{CeO}_{2}\left(0\right.$ and $78 \% \mathrm{Zr}$ ) and $\mathrm{Co}_{3} \mathrm{O}_{4}\left(25\right.$ and $\left.75 \% \mathrm{Fe}_{3} \mathrm{O}_{4}\right)$ NPs caused significant increases in eosinophil density compared to OVA alone (Figure 7c). 

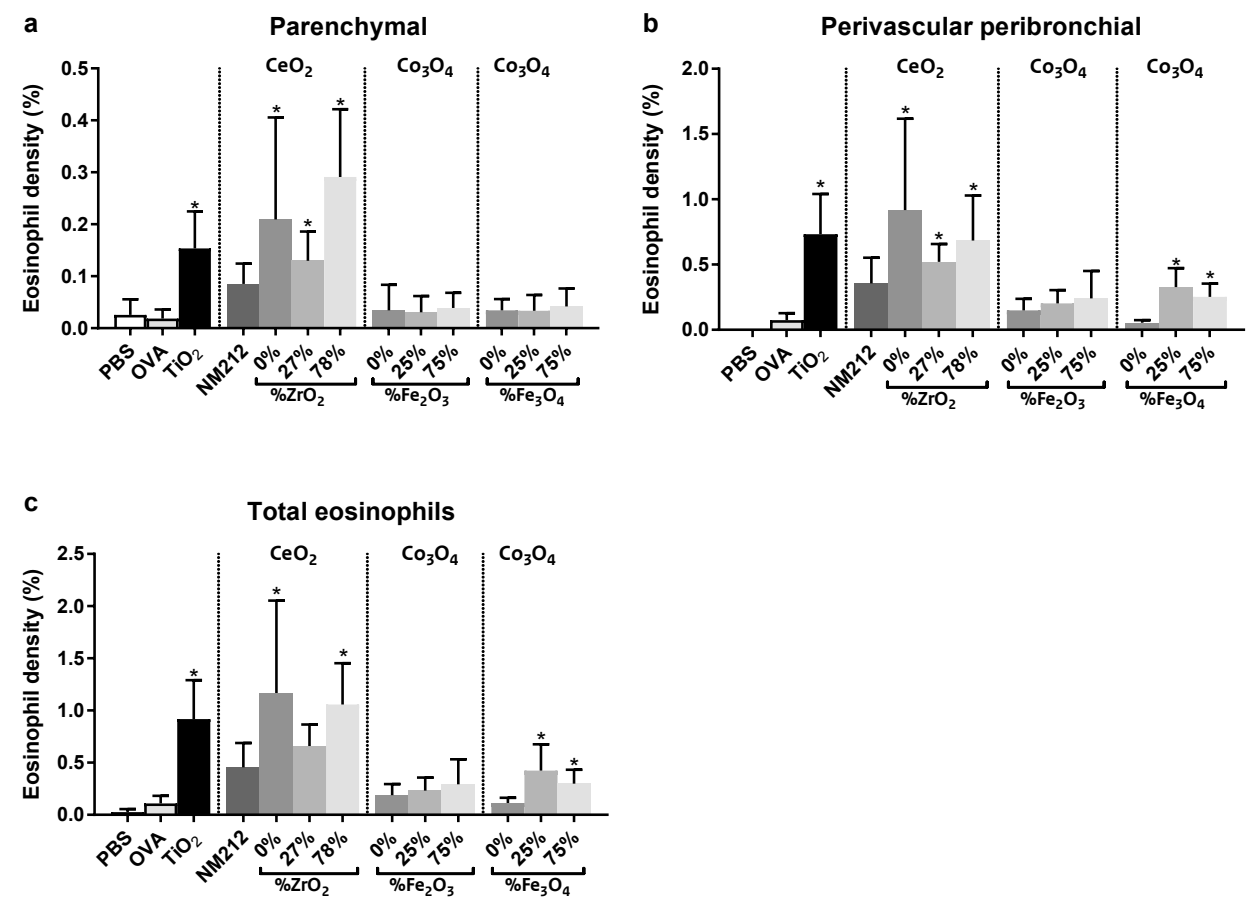

Figure 7: Eosinophil density.

Quantification of the eosinophil density in the parenchymal (a), perivascular and peribronchial (b), and total (c) lung tissue. Mean $\pm S D, n=6$ except OVA where $n=8,{ }^{*}=$ statistically significant different from OVA controls $(p<0.05)$.

\section{B-lymphocytes density}

For quantification of the B-lymphocytes density lung tissue sections were stained for CD45R, a marker for B-lymphocytes.

Co-sensitization with OVA and $\mathrm{Co}_{3} \mathrm{O}_{4}$ NPs induced a severe accumulation of B-lymphocytes (CD45+ cells) in the perivascular/peribronchiolar interstitium, indicative for a chronic inflammatory response. By comparison, accumulation of B-lymphocytes in lungs from OVA plus $\mathrm{CeO}_{2}$ NP-sensitised mice was not as evident (Figure 8). 


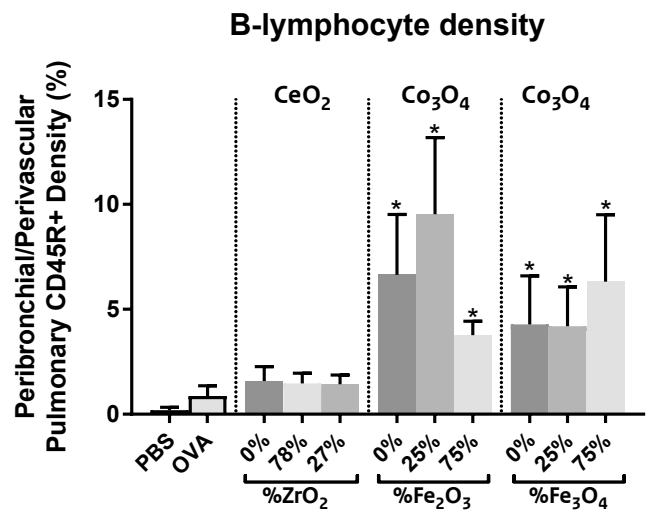

Figure 8: B-lymphocyte density.

Quantification of the B-lymphocyte density in the perivascular and peribronchial lung tissue. Mean $\pm S D, n=6$ except OVA where $n=8, *=$ statistically significant different from OVA controls $(p<0.05)$.

\section{Lung histopathology}

Examples of histopathological findings in the scanned and digitized histologic slides of the lung tissue sections are shown in Figure 9 and 10. No exposure related microscopically observable lung lesions were found in the PBS control group (Figure 9a and b). All mice that were intranasally sensitized and challenged with OVA (OVA controls) had some degree of histopathology in the lung that was characteristic of allergic airway disease. This histopathology consisted of a mixed inflammatory cell influx containing eosinophils, lymphoid cells (lymphocytes and plasma cells) and, to a lesser extent, neutrophils in the interstitial tissue surrounding blood vessels and conducting airways (perivascular and peribronchiolar inflammation) (most conspicuous in G5, see Figure 9c, but also in G11). Associated with these inflammatory lesions, there was mucous cell metaplasia of the airway epithelium (appearance of mucous cells with AB/PAS-stained mucosubstances) (see Figure 9d).

Mice co-exposed to OVA and NPs had a mild to moderate multifocal accumulation of particleladen, hypertrophic macrophages in alveolar air spaces (most conspicuous in centriacinar regions of the lung) and more severe inflammatory or epithelial lung lesions compared to mice exposed to OVA alone. Mice co-exposed to OVA and $\mathrm{TiO}_{2}, \mathrm{CeO}_{2} \mathrm{NM} 212$, or $\mathrm{Zr}$-doped $\mathrm{CeO}_{2}$ NPs had more severe lymphocytic and eosinophilic inflammation as (see Figure 9e) as well as more severe mucous cell metaplasia (most conspicuous in G11, see Figure 9f) compared to the OVA-alone treated mice (see Figure 9c and d). 

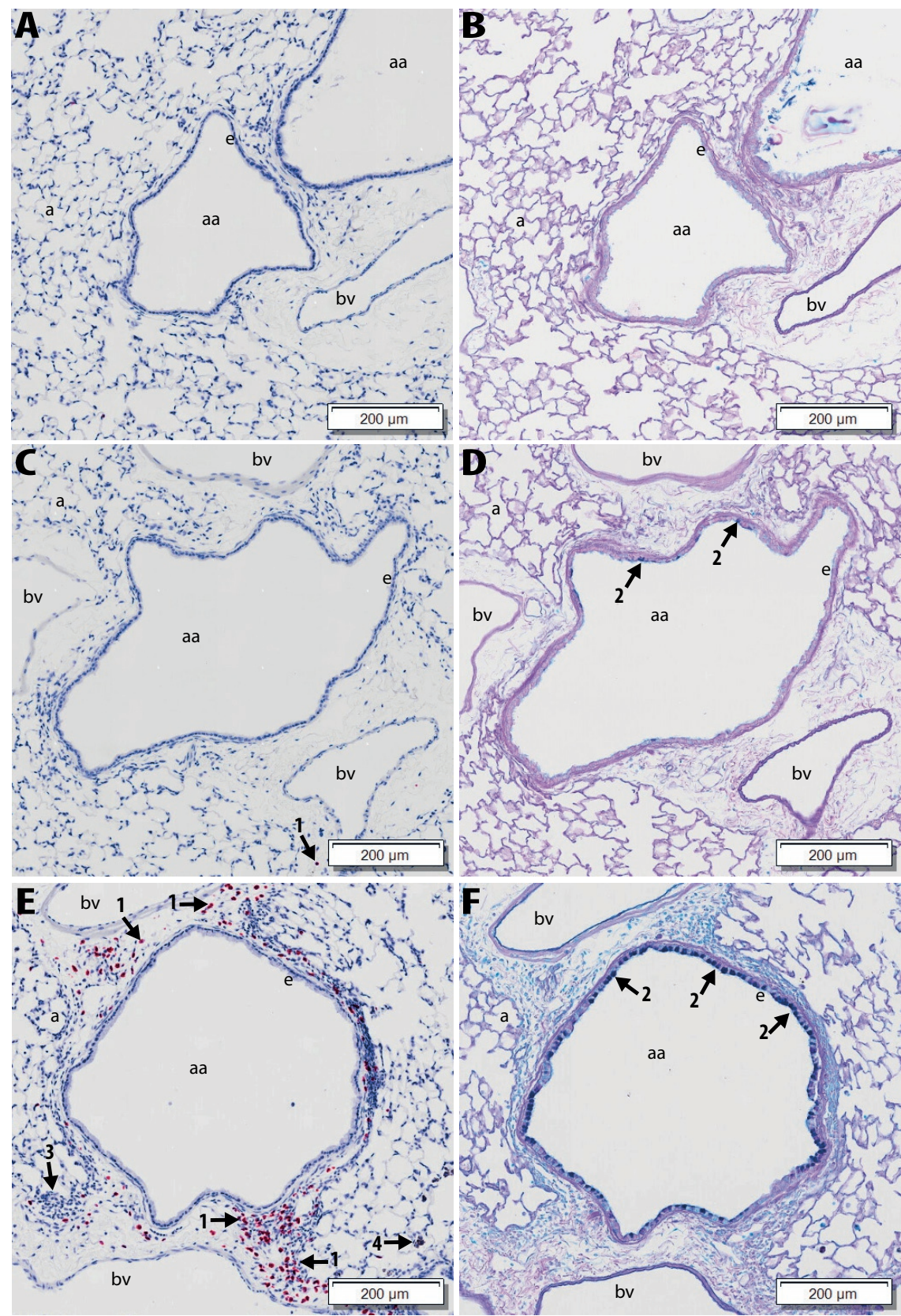

Figure 9: Examples of observed histopathological findings in lungs.

Light photomicrographs of G11, small-diameter, distal axial airway from the lungs of PBS-treated mice (A, B), OVAtreated mice $(C, D)$ and $O V A+T_{i O}$ NP treated mice $(E, F)$. Lung tissue sections for $A, C$ and $E$ were stained for murine major basic protein, a specific marker for eosinophils (red chromogen-stained cells; arrow 1). Tissue sections $B$, $D$ and $F$ were stained with Alcian Blue/Periodic Acid Schiff (AB/PAS) for neutral and acidic mucosubstances (dark magenta stain; arrow 2) in mucous cells of the airway epithelium (e). aa: axial airway lumen; bv: blood vessel; a: alveolus; arrow 3:mononuclear cell infiltrate (mainly lymphoid) ; arrow 4: $\mathrm{TiO}_{2} \mathrm{NP}$-laden alveolar macrophages 
In contrast, OVA and $\mathrm{CO}_{3} \mathrm{O}_{4}$ (with or without Fe-doping) co-exposed mice did not have more severe eosinophilic inflammation or mucous cell metaplasia as compared to OVAalone treated mice. Interestingly, these OVA and $\mathrm{CO}_{3} \mathrm{O}_{4}$ co-exposed mice exhibited more severe perivascular or peribronchiolar lymphoid cell accumulation compared to mice coexposed to the other NPs, organized in ectopic lymphoid-like structures (ELS, see Figure $10 \mathrm{a}, \mathrm{b}$ and $\mathrm{c}$ ). ELS are tertiary lymphoid organs, associated for example with infections and autoimmune diseases, that develop in areas of chronic inflammation and are characterized by the formation of organized B- and T-cell aggregates and germinal centres with follicular dendritic cell networks ${ }^{[23,24]}$. These lung lesions were also observed in mice co-exposed to OVA and $\mathrm{Co}_{3} \mathrm{O}_{4}$ with Fe-doping (using both $\mathrm{Fe}_{2} \mathrm{O}_{3}$ and $\mathrm{Fe}_{3} \mathrm{O}_{4}$, see Figure 10d, e and f).
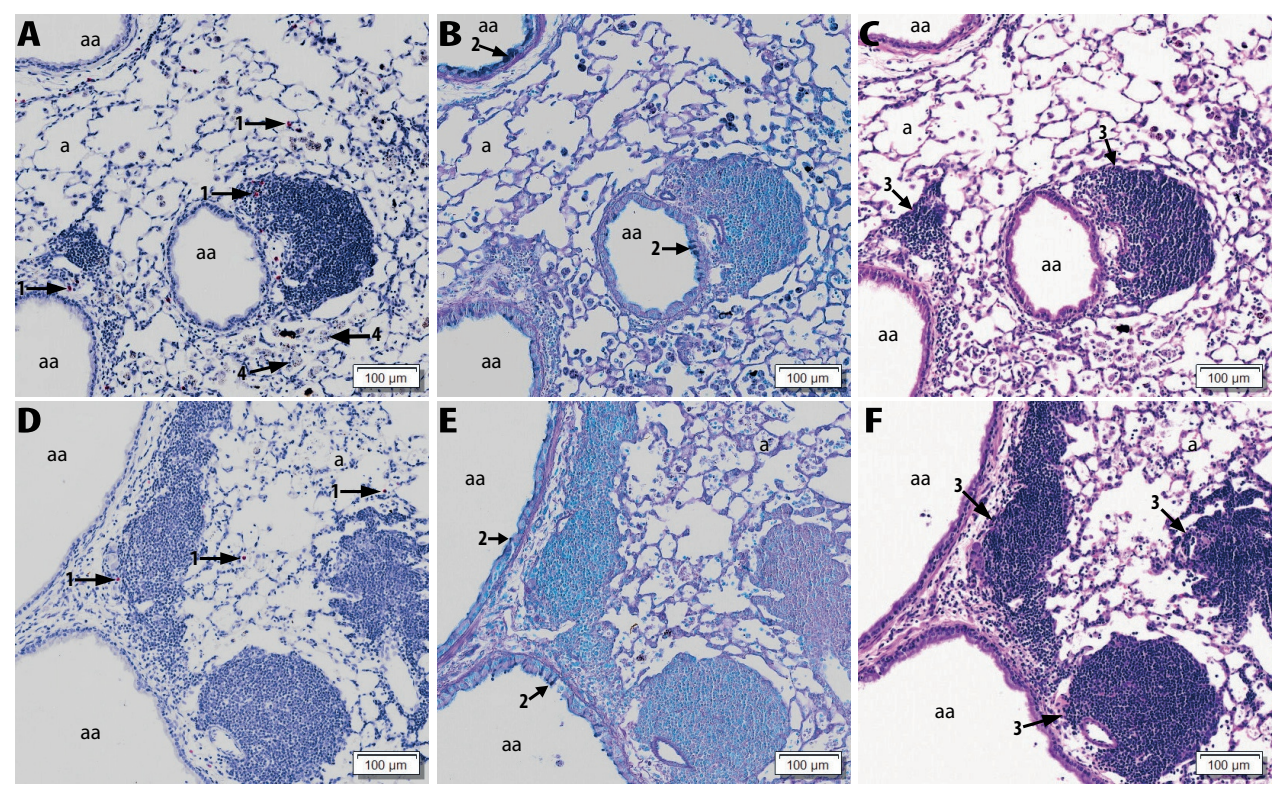

Figure 10: Examples of observed ectopic lymphoid structures in lungs.

Light photomicrographs of $\mathrm{G} 5$, principally large-diameter, proximal axial airway from the lungs of OVA+Co $\mathrm{O}_{4}(0 \%$ $\left.\mathrm{Fe}_{2} \mathrm{O}_{3}\right) \mathrm{NP}$ treated mice $(\mathrm{A}, \mathrm{B}, \mathrm{C})$, and OVA+ $\mathrm{Co}_{3} \mathrm{O}_{4}\left(25 \% \mathrm{Fe}_{2} \mathrm{O}_{3}\right)$ NP-treated mice $(\mathrm{D}, \mathrm{E}, \mathrm{F})$. Lung tissue sections for $\mathrm{A}$ and $D$ were stained for murine major basic protein, a specific marker for eosinophils (red chromogen-stained cells; arrow 1). Tissue sections B and E were stained with Alcian Blue/Periodic Acid Schiff (AB/PAS) for neutral and acidic mucosubstances (dark magenta stain; arrow 2) in mucous cells of the airway epithelium. Tissue sections $\mathrm{C}$ and $\mathrm{F}$ were stained with hematoxylin and eosin (H\&E). arrow 3: ectopic lymphoid structures (ELS), arrow 4: $\mathrm{Co}_{3} \mathrm{O}_{4} \mathrm{NP}-$ laden alveolar macrophages; aa: axial airway lumen; a: alveolus.

\section{Discussion}

All NPs investigated possessed adjuvant activity, leading to changes in OVA-specific IgE and IgG1 plasma levels, differential cell count and cytokines in BALF, and histopathological detection of mucosa cell metaplasia and eosinophil density in the conducting airways. Coexposure of OVA and $\mathrm{Co}_{3} \mathrm{O}_{4} \mathrm{NPs}$ also induced perivascular and peribronchiolar lymphoid cell accumulation and the formation of ELS in lungs. 


\section{Adjuvant activity}

Although the increased OVA-specific IgE and IgG1 plasma levels of all OVA + NP exposed groups compared to OVA controls were not statistically significant for all NPs, they do indicate that NPs have the capacity to enhance allergic sensitisation in mice. This is supported by the increases in eosinophils, lymphocytes, IL-4, IL-5 and IL-6 in BALF after co-exposure of OVA and many NPs compared to the OVA controls. In our previous study, we found that $\mathrm{SiO}_{2}$ and $\mathrm{TiO}_{2} \mathrm{NP}$ exposure alone (without OVA) during the induction phase did not lead to an increase in OVA-specific IgE and IgG1 plasma levels or eosinophils in BALF after OVA challenge ${ }^{[9,10]}$. These findings suggest that the allergic response can only be enhanced via co-exposure of the allergen and NPs, and not by exposure to NPs alone. In the current study, all NPs were only tested in combination with OVA, and the results show that all NPs were able to act as adjuvant, albeit to varying degrees.

\section{The influence of the chemical composition}

The differences in OVA-specific IgE and IgG1 plasma levels of the animals co-sensitized to OVA and the different NPs indicate that the chemical composition of the NPs influences their potency to exacerbate allergic airway sensitization. The relative adjuvant potencies of the different NPs varied with the different biomarkers of effects (Figure 11), indicating that the chemical composition also influences the type of the allergic response.

\begin{tabular}{|c|c|c|c|c|c|c|c|}
\hline \multirow{2}{*}{$\begin{array}{c}\text { Biomarkers } \\
\text { of effect }\end{array}$} & \multirow{2}{*}{$\begin{array}{c}\text { OVA } \\
\text { controls }\end{array}$} & \multicolumn{5}{|c|}{ Chemical composition of NPs } & \multirow[b]{3}{*}{125} \\
\hline & & $\mathrm{TiO}_{2}$ & $\begin{array}{c}\mathrm{CeO}_{2} \\
\text { (NM212) }\end{array}$ & $\begin{array}{c}\mathrm{CeO}_{2} \\
\left(0 \% \mathrm{ZrO}_{2}\right)\end{array}$ & $\begin{array}{c}\mathrm{Co}_{3} \mathrm{O}_{4} \\
\left(0 \% \mathrm{Fe}_{2} \mathrm{O}_{3}\right)\end{array}$ & $\begin{array}{c}\mathrm{Co}_{3} \mathrm{O}_{4} \\
\left(0 \% \mathrm{Fe}_{3} \mathrm{O}_{4}\right)\end{array}$ & \\
\hline $\lg \mathrm{E}$ & & & & & & & \\
\hline $\lg \mathbf{l g} 1$ & & & & & & & 30000 \\
\hline Total cell count BALF & & & & & & & 175000 \\
\hline$\%$ Neutrophils BALF\% & & & & & & & 35 \\
\hline Eosinophils BALF\% & & & & & & & 7 \\
\hline Lymphocytes BALF & & & & & & & 7 \\
\hline IL-4 in BALF & & & & & & & 7 \\
\hline IL-5 in BALF & & & & & & & 15 \\
\hline IL-6 in BALF & & & & & & & 25 \\
\hline IL-13 in BALF & & & & & & & 30 \\
\hline Mucosubstance G5 & & & & & & & 2.5 \\
\hline Mucosubstance G11 & & & & & & & 1.5 \\
\hline B-lymphocyte density & & & $><$ & & & & 4 \\
\hline Eosinophilic density & & & & & & & 0.7 \\
\hline
\end{tabular}

Figure 11: The influence of the chemical composition on the different biomarkers of effect.

The shading indicates the lowest (light grey), intermediate (medium grey) or highest (dark grey) response of an NP (relative to the OVA controls) for each of the biomarkers. The bar on the right side shows for each row the value corresponding with the indicated (medium) grey shading. 


\section{Type of the immune responses}

Animals co-exposed to OVA and undoped $\mathrm{CeO}_{2}$ NPs showed higher OVA-specific IgE plasma levels compared to animals co-exposed to OVA and the other undoped NPs ( $\mathrm{TiO}_{2} \mathrm{NPs}$, $\mathrm{CeO}_{2} \mathrm{NM} 212 \mathrm{NPs}$, and $\mathrm{Co}_{3} \mathrm{O}_{4} \mathrm{NPs}$ ), indicative for a type 1 (immediate or IgE mediated) allergic response. Additionally, indications for a Th2 type immune response were observed, characterised by an increase in the percentage of eosinophils and increased concentrations of the Th2 cytokines IL-4 and IL-5 (but not IL-13) in the BALF. Animals co-exposed to OVA and the OECD representative manufactured nanomaterial $\mathrm{CeO}_{2}$ (NM212), also showed some indications of a Th2 type response, albeit less marked than those observed in animals co-exposed to OVA and $\mathrm{CeO}_{2}(0 \% \mathrm{Zr})$ NPs: plasma OVA-specific IgE levels, percentage of eosinophils in BALF and BALF IL-4 concentrations were much lower in animals co-exposed to OVA and $\mathrm{CeO}_{2} \mathrm{NM} 212$ than in animals co-exposed to OVA and $\mathrm{CeO}_{2}(0 \% \mathrm{Zr}) \mathrm{NPs}$. In contrast, concentrations of IL-5 in BALF were similarly affected by both forms of $\mathrm{CeO}_{2}$. These findings

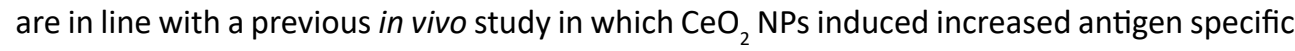
IgE levels, eosinophils, Th2 cytokines and Th2 chemokines when co-exposed with house dust mite ${ }^{[25]}$ and an in vitro study in which $\mathrm{CeO}_{2} \mathrm{NPs}$ exposure of human dendritic cells induced a Th2-dominated cell profile ${ }^{[26]}$.

Co-exposure to OVA and undoped $\mathrm{Co}_{3} \mathrm{O}_{4}$ NPs also showed responses indicative of a type 1 allergic response, albeit, the OVA-specific IgE plasma levels were lower compared to coexposure to OVA and the $\mathrm{CeO}_{2}(0 \% \mathrm{Zr}) \mathrm{NPs}$. Furthermore, the response after co-exposure to OVA and $\mathrm{Co}_{3} \mathrm{O}_{4}$ NPs was characterised by no or less marked increases in in BALF IL-4 and IL-5 concentrations and the percentage of eosinophils in BALF, but a more pronounced increase in the BALF IL- 6 concentration and the percentage of lymphocytes in BALF compared to coexposure to OVA and the $\mathrm{CeO}_{2}(0 \% \mathrm{Zr})$ NPs. This pattern of response is in line with a previous in vivo study in $\mathrm{C} 57 \mathrm{BI} / 6$ mice in which subcutaneous administration of $\mathrm{CO}_{3} \mathrm{O}_{4}$ NPs with OVA induced both Th1 and Th2 type responses ${ }^{[27]}$. Several biomarkers, including the OVA-specific IgG1 plasma levels and IL-6 concentrations in the BALF, were remarkably different in animals co-exposed to OVA and the two different types of undoped $\mathrm{Co}_{3} \mathrm{O}_{4}$ NPs, indicating that not only chemical composition, but also other physicochemical characteristics of the NPs are of influence to the allergic response. Although, the production process of the two undoped $\mathrm{Co}_{3} \mathrm{O}_{4}$ NPs was the same, they differed in the degree of crystallinity, aggregation, $\mathrm{Co} / \mathrm{Fe}$ ratio and spatial distributions of the metals in the NPs ${ }^{[18]}$.

The immune response after co-exposure to $\mathrm{OVA}_{\text {and }} \mathrm{TiO}_{2} \mathrm{NPs}_{2}$ was indicative for a Th2 immune response, characterised by an increase in eosinophils, IL-4 and IL-5 in BALF. Previous studies with $\mathrm{TiO}_{2}$ NPs have found contradictory results regarding effects on immune responses. Schanen et al. ${ }^{[26]}$ found that exposure of dendritic cells to $\mathrm{TiO}_{2} \mathrm{NPs}$ led towards Th1-based responses, while Vandebriel et al. ${ }^{[10]}$ and de Haar et al. ${ }^{[28]}$ observed Th2 type immune responses in mice after co-exposure to $\mathrm{OVA}_{\text {plus }} \mathrm{TiO}_{2} \mathrm{NPs}$. However, the contradictory result 
may have been caused by differences in the physicochemical characteristics (e.g. sizes and/ or crystallinity) of the $\mathrm{TiO}_{2}$ NPs used in the studies by Schanen et al. ${ }^{[26]}$ and de Haar et al. ${ }^{[28]}$, compared to the $\mathrm{TiO}_{2}$ NPs used in our study, which were the same $\mathrm{TiO}_{2} \mathrm{NPs}$ as Vandebriel et $a{ }^{\left[{ }^{[10]}\right.}$. Although this study adds weight to the steering of the immune response towards a Th2 response by $\mathrm{TiO}_{2}$ NP co-exposure with antigens, more studies which measure a wide range of Th2 and Th1/Th17 associated biomarkers are needed to gain more insight in the type of adjuvant activity associated with by $\mathrm{TiO}_{2} \mathrm{NPs}$ exposure.

\section{Lung morphology and ectopic lymphoid-like structures}

Animals co-exposed to OVA and $\mathrm{TiO}_{2}, \mathrm{CeO}_{2} \mathrm{NM} 212$, or $\mathrm{CeO}_{2}(0 \% \mathrm{Zr}) \mathrm{NPs}$ had more severe mucous cell metaplasia (most conspicuous in G11, but also in G5), as well as more severe eosinophilic inflammation, compared to the OVA-alone treated mice. OVA and $\mathrm{CO}_{3} \mathrm{O}_{4}$ co-exposed mice showed more severe perivascular and peribronchiolar lymphoid cell accumulation, organized into ELS. These findings were accompanied by an increase in lymphocytes in BALF. In mice broncho-associated lymphoid tissue (BALT) is usually absent, however, it can be induced (inducible BALT or iBALT) by inflammatory processes and infections ${ }^{[29]}$. In humans, iBALT formation can be observed in the lung in response to various types of infectious and inflammatory states, caused by infectious organisms, diesel exhaust, cigarette smoke silica, and various autoimmune diseases ${ }^{[30,31]}$. In previous studies lymphocyte foci or aggregates have been observed in lungs of rats after intratracheal instillation of $\mathrm{CO}_{3} \mathrm{O}_{4} \mathrm{NPS}^{[32]}$, as well as in lungs of mice after intranasal exposure to OVA ${ }^{[33]}$, crystalline silica particles $(1.5-2.0 \mu \mathrm{m})^{[34]}$ and after inhalation of CdO NPs ${ }^{[35]}$. However, the role of iBALT in the pathophysiology of chronic allergic diseases, such as asthma, is poorly understood ${ }^{[30,31]}$. Studies investigating whether or not iBALT is involved in the development or progression of allergy or asthma showed conflicting results and the pathways that control the development and function of iBALT are poorly understood.

\section{The influence of redox modification by doping}

The differences in immune responses observed between the groups exposed to the same type of NPs, but different amounts of doping (Zr-doping for $\mathrm{CeO}_{2} \mathrm{NPs}$ and Fe-doping for $\mathrm{Co}_{3} \mathrm{O}_{4} \mathrm{NPs}$ ), indicate that redox modification can influence the adjuvant activity of NPs. However, the amount of doping did not always show a clear trend in the response of the different biomarkers of effects. Some biomarkers indicated that the undoped NPs exhibit most adjuvant activity, while other biomarkers indicated that either the NP with the lowest amount of doping or the NPs with the highest amount of doping exhibited most adjuvant activity. Additionally, most biomarkers of effects neither decreased nor increased with greater degrees of doping (see Additional file 3).

This lack of trend between increasing amounts of doping and the biomarkers of effects is also observed in the EPR data, in which only the $\mathrm{Co}_{3} \mathrm{O}_{4}\left(0,25\right.$ and $\left.75 \% \mathrm{Fe}_{3} \mathrm{O}_{4}\right)$ NPs showed 
a decreasing scavenging capacity with increasing amounts of doping (see Figure 2). This decrease in free hydroxyl radical scavenging capacity was unexpected based on the Ec levels of $\mathrm{CO}_{3} \mathrm{O}_{4}$ and $\mathrm{Fe}_{3} \mathrm{O}_{4}$. We expected that doping using $\mathrm{Fe}_{3} \mathrm{O}_{4}$ would increase, rather than decrease the ability of $\mathrm{Co}_{3} \mathrm{O}_{4}$ to scavenge free hydroxyl radicals, because the Ec level of $\mathrm{Co}_{3} \mathrm{O}_{4}$ overlaps with the cellular redox potential, whereas that of $\mathrm{Fe}_{3} \mathrm{O}_{4}$ does not. On the other hand, the superoxide generation did increase with Fe-doping of $\mathrm{Co}_{3} \mathrm{O}_{4} \mathrm{NPs}$ using $25 \% \mathrm{Fe}_{3} \mathrm{O}_{4}$, but this ROS generation was not further increased with increasing amounts of Fe-doping. It is possible that differences in particle size and aggregation counterbalanced any dopingrelated differences in free radical generation or scavenging ${ }^{[11,36]}$, as the particle size in water as measured with disc centrifuge was larger for the $\mathrm{Co}_{3} \mathrm{O}_{4}\left(75 \% \mathrm{Fe}_{3} \mathrm{O}_{4}\right)$ NPs compared to $\mathrm{CO}_{3} \mathrm{O}_{4}\left(25 \% \mathrm{Fe}_{3} \mathrm{O}_{4}\right) \mathrm{NPs}$, although the difference in particle size between these two NPs was relatively small. Nonetheless, we would still have expected more notable differences in free radical generation from the surfaces of the differently doped materials. Physicochemical characterisation of the materials indicates successful incorporation of the doping into the crystal structure of the NP ${ }^{[18]}$, thus at present we can only speculate as to the reason for the limited effect of doping on free radical generation.

Since, the amount of doping or band gap energy of the NPs were not predictive for adjuvant activity or the ability to generate or scavenge specific radical, we also investigated if the ability of the NPs to generate or scavenge specific radicals was predictive for the adjuvant activity.

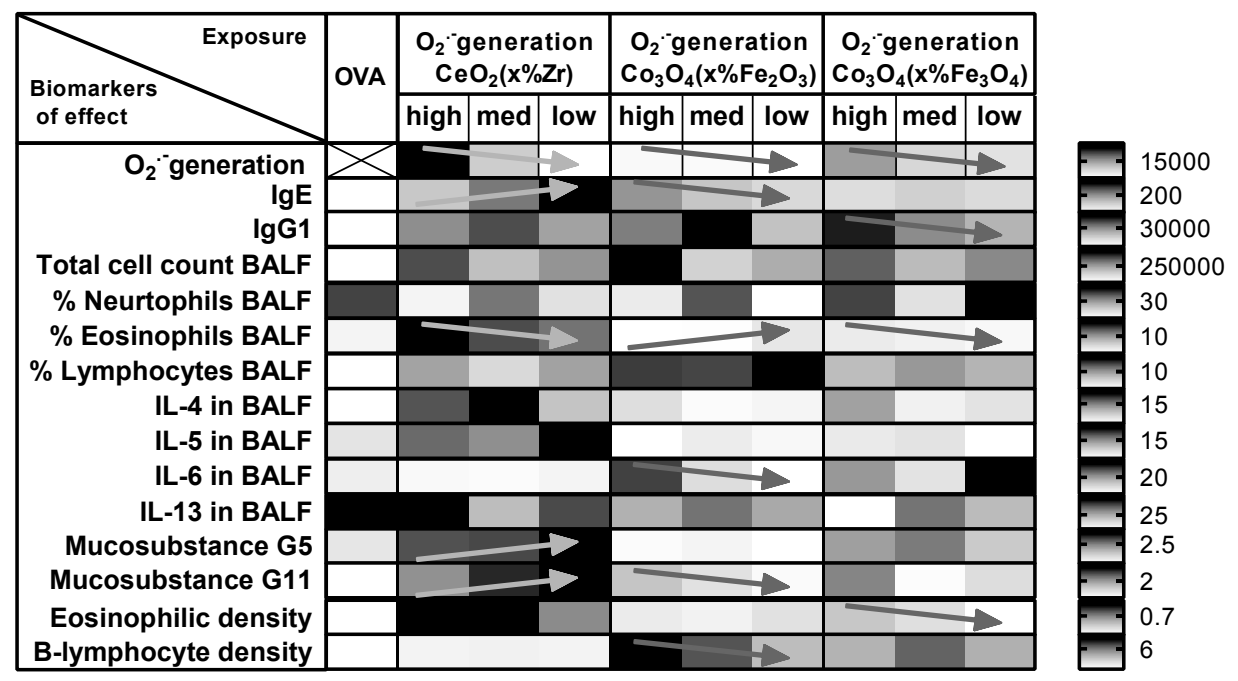

Figure 12: The influence of the ability of NPs to induce superoxide generation as measured in an acellular EPR assay on the different biomarkers of effects.

The shading indicates the lowest (light grey) to highest (dark grey) response of an NP (relative to each other) for each of the biomarkers. The arrows indicate an increase $(\lambda)$ or decrease $(\checkmark)$ with decreasing amount of superoxide generation. The bar on the right side shows for each row the value corresponding with the indicated (medium) grey shading. 
The superoxide generation as measured with the EPR was not predictive for the adjuvant activity. Only a few biomarkers of effects decreased with decreasing amounts of superoxide generation and these biomarkers of effects were different for each of the NP series (see arrows in Figure 12).

The scavenging capacity as measured with the EPR was also not predictive for the adjuvant activity. Only a few biomarkers of effects decreased with increasing amounts of scavenging capacity and these biomarkers of effects were different for each of the NP series (see arrows in Figure 13).

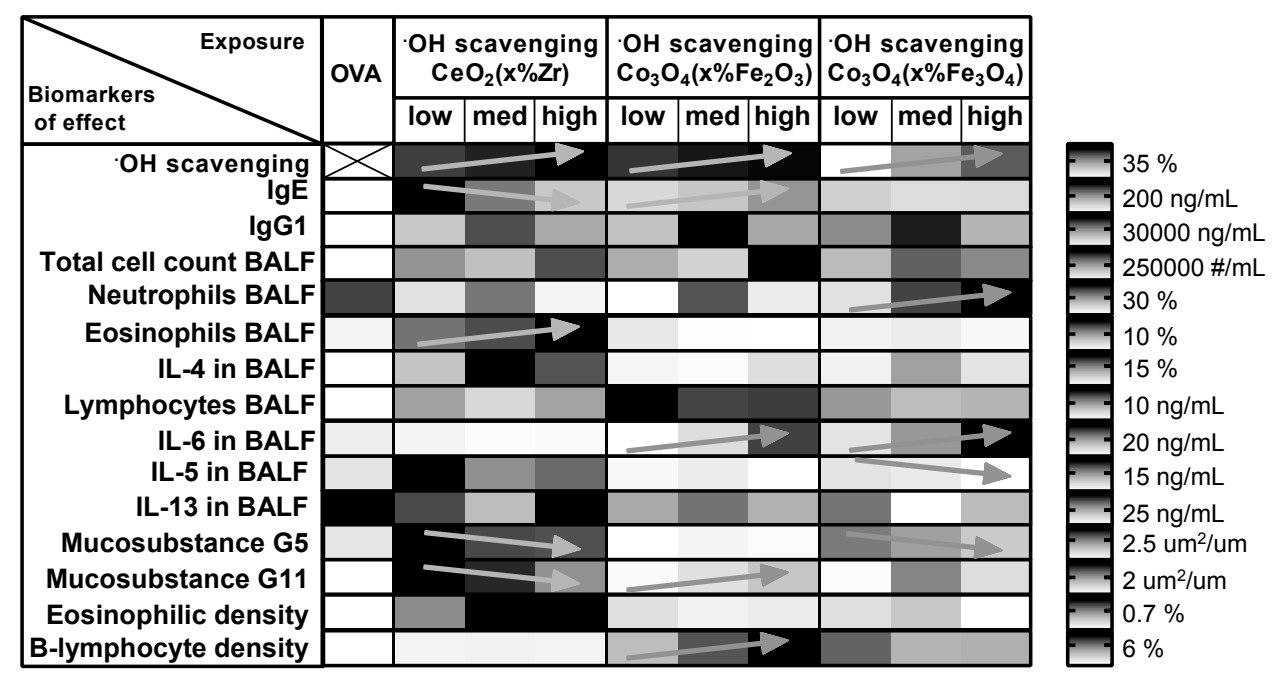

Figure 13: The influence of the scavenging capacity of the NPs as measured in an acellular EPR assay on the different biomarkers of effects.

The shading indicates the lowest (light grey) to highest (dark grey) response of an NP (relative to each other) for each of the biomarkers. The arrows indicate an increase $(\lambda)$ or decrease $(\)$ with increasing scavenging capacity. The bar on the right side shows for each row the value corresponding with the indicated (medium) grey shading.

While EPR is a useful technique to predict the ability of NPs to generate cellular oxidative stress, this acellular approach provides only a facet of the redox reactivity of NPs within cellular compartments. However, due to the number of NPs under investigation, a comprehensive comparison of redox activity in cellular assays was not possible within the current study. Therefore, a possible explanation for the lack of trend between the EPR results and the adjuvant activity is that redox activity of the NPs may change due to interaction with the physiological environment, e.g. by corona formation, aggregation, elimination, redox reactions, etc. Interactions with the physiological environment are known to influence the reactivity, cellular uptake and toxicity of the NPs ${ }^{[37-40]}$. Han et al. ${ }^{[41]}$ found that co-exposure of OVA and polyethylene glycol-conjugated (PEGylated) $\mathrm{SiO}_{2} \mathrm{NPs}$ induced less severe airway inflammation compared to non-coated $\mathrm{SiO}_{2} \mathrm{NPs}$. It was suggested that 
this was due to an increased aggregation of the PEGylated $\mathrm{SiO}_{2} \mathrm{NPs}$ in saline compared to the non-coated $\mathrm{SiO}_{2}$. However, the small differences in the (aggregated) particle diameter of the NPs in water observed in our study (see Table 2) would be very unlikely to significantly influence adjuvant potency. Larger differences in the (aggregated) particle diameter of the NPs were observed in a more physiologically relevant environment (i.e. buffered cell culture medium with proteins from fetal calf serum) (see Table 2). However, no relation between these differences in aggregated size and the adjuvant potencies of the different NPs found. Horie et al. ${ }^{[42]}$ found differences in OVA-specific IgE and IgG1 concentrations in the blood of mice exposed to $\mathrm{ZnO}$ compared to $\mathrm{SiO}_{2}$-coated $\mathrm{ZnO}$ NPs after subsequent exposure to OVA. The investigators suggested that these differences might be due to differences in solubility and continuous $\mathrm{Zn}^{2+}$ release from the NPs. Although the NPs in our study are expected to be relatively insoluble, further evaluation of the possible degradation and ion release under various physiological conditions (e.g. in lysosomal fluid) are needed to rule out this possibility. Further research is needed to better understand the mechanisms of the adjuvant activity of NPs, including the influence of the NP properties and NP interactions with their physiological environment, including allergens (such as OVA), on the adjuvant activity.

\section{Conclusions}

The chemical composition of NPs in this study influenced both their relative potency to exacerbate allergic airway sensitization to ovalbumin (OVA), and the type of the immune response. The adjuvant activity of $\mathrm{CeO}_{2} \mathrm{NPs}$ seems to be primarily mediated via a Th2-type immune response, whereas the adjuvant activity of $\mathrm{Co}_{3} \mathrm{O}_{4} \mathrm{NPs}$ was characterised by no or less marked increases in OVA-specific IgE plasma levels, BALF IL-4 and IL-5 concentrations and percentages of eosinophils in BALF and more pronounced increases in the BALF IL- 6 concentrations and percentages of lymphocytes in BALF. Co-exposure to OVA and $\mathrm{Co}_{3} \mathrm{O}_{4}$ NPs also induced lymphoid cell accumulation and the formation of ectopic lymphoid tissues in the lungs. No relation between the acellular redox potential and the observed adjuvant activity of the different of NPs was found, suggesting that acellular ROS assays may not be suitable to predict adjuvant capacity. These findings highlight the complexity of biological interplay between different NP species and the need for additional data and methods to formulate scenarios that better predict the adjuvant activity of nanomaterial families.

\section{Abbreviations}

NP: Nanoparticle; OVA: ovalbumin; EPR: Electron Paramagnetic Resonance; STEM: Scanning Transmission Electron Microscopy; ROS: Reactive Oxygen Species; Ec: Conduction band energy level; BALF: Bronchoalveolar Lavage Fluid; PBS: Phosphate-Buffered Saline; MBP: Major Basic Protein; ANOVA: analysis of variance; SD: Standard Deviation; RT: Room Temperature; LAL: Limulus Amoebocyte Lysate; DMPO: 5,5-dimethyl-1-pyrroline N-oxide. 


\section{Funding}

The work leading to these results has received funding from the European Union's Seventh Framework Programme for research, technology development and demonstration under grant agreement $n^{\circ} 310451$ (NanoMILE) and the Netherlands Food and Consumer Product Safety Authority (V090016) (NVWA). MRM is supported by a British Heart Foundation (BHF) Special Project Grant (SP/15/8/31575).

\section{Acknowledgements}

The authors would like to thank Ryan Lewandowski and Amy Freeland of MSU for their help during the conduction of the animal experiment and valuable technical assistance, Amy Porter, Kathleen Joseph and William Lienhart of MSU Investigative Histopathology Laboratory for their histotechnology support, Daan Leseman and Eric Gremmer of RIVM for their support in the characterizing the particles and for the IgG and IgE ELISA and cytokine analyses of the BALF, respectively, Ed Lester of Promethean Particles for his contribution to the design and production of the particles, Christina Giannakou of RIVM for the LCMS-MS analysis of the endotoxin levels, and Eva Valsami-Jones of UoB coordinator of the NanoMILE project. 


\section{References}

1. Cho WS, Duffin R, Thielbeer F, Bradley M, Megson IL, Macnee W, Poland CA, Tran CL, Donaldson K: Zeta potential and solubility to toxic ions as mechanisms of lung inflammation caused by metal/ metal oxide nanoparticles. Toxicol Sci 2012, 126:469-477.

2. Dekkers S, Oomen AG, Bleeker EA, Vandebriel RJ, Micheletti C, Cabellos J, Janer G, Fuentes N, Vazquez-Campos S, Borges $\mathrm{T}$, et al: Towards a nanospecific approach for risk assessment. Regul Toxicol Pharmacol 2016, 80:46-59.

3. Teunenbroek TV, Baker J, Dijkzeul A: Towards a more effective and efficient governance and regulation of nanomaterials. Part Fibre Toxicol 2017, 14:54.

4. Zhang H, Ji Z, Xia T, Meng H, Low-Kam C, Liu R, Pokhrel S, Lin S, Wang X, Liao YP, et al: Use of metal oxide nanoparticle band gap to develop a predictive paradigm for oxidative stress and acute pulmonary inflammation. ACS Nano 2012, 6:4349-4368.

5. Miller MR, Shaw CA, Langrish JP: From particles to patients: oxidative stress and the cardiovascular effects of air pollution. Future Cardiol 2012, 8:577-602.

6. Unfried KA, Catrin; Klotz, Lars-Oliver; Von Mikecz, Anna; Grether-Beck, Susanne; Schins, Roel P.F. : Cellular responses to nanoparticles: Target structures and mechanisms. Nanotoxicology 2007, 1:52-71.

7. Meldrum K, Guo C, Marczylo EL, Gant TW, Smith R, Leonard MO: Mechanistic insight into the impact of nanomaterials on asthma and allergic airway disease. Part Fibre Toxicol 2017, 14:45.

8. Pisoschi AM, Pop A: The role of antioxidants in the chemistry of oxidative stress: A review. Eur J Med Chem 2015, 97:55-74.

9. Brandenberger C, Rowley NL, Jackson-Humbles DN, Zhang Q, Bramble LA, Lewandowski RP, Wagner JG, Chen W, Kaplan BL, Kaminski NE, et al: Engineered silica nanoparticles act as adjuvants to enhance allergic airway disease in mice. Part Fibre Toxicol 2013, 10:26.

10. Vandebriel RJ, Vermeulen JP, van Engelen LB, de Jong B, Verhagen LM, de la Fonteyne-Blankestijn $\mathrm{LJ}$, Hoonakker ME, de Jong WH: The crystal structure of titanium dioxide nanoparticles influences immune activity in vitro and in vivo. Part Fibre Toxicol 2018, 15:9.

11. Fu PP, Xia Q, Hwang HM, Ray PC, Yu H: Mechanisms of nanotoxicity: generation of reactive oxygen species. J Food Drug Anal 2014, 22:64-75.

12. Khanna P, Ong C, Bay BH, Baeg GH: Nanotoxicity: An Interplay of Oxidative Stress, Inflammation and Cell Death. Nanomaterials (Basel) 2015, 5:1163-1180.

13. Nel A, Xia T, Madler L, Li N: Toxic potential of materials at the nanolevel. Science 2006, 311:622627.

14. Dekkers S, Miller MR, Schins RPF, Romer I, Russ M, Vandebriel RJ, Lynch I, Belinga-Desaunay MF, Valsami-Jones $\mathrm{E}$, Connell SP, et al: The effect of zirconium doping of cerium dioxide nanoparticles on pulmonary and cardiovascular toxicity and biodistribution in mice after inhalation. Nanotoxicology 2017, 11:794-808. 
15. Zhang H, Pokhrel S, Ji Z, Meng H, Wang X, Lin S, Chang CH, Li L, Li R, Sun B, et al: PdO doping tunes band-gap energy levels as well as oxidative stress responses to a $\mathrm{Co}(3) \mathrm{O}(4) \mathrm{p}$-type semiconductor in cells and the lung. J Am Chem Soc 2014, 136:6406-6420.

16. Kroker M, Sydlik U, Autengruber A, Cavelius C, Weighardt H, Kraegeloh A, Unfried K: Preventing carbon nanoparticle-induced lung inflammation reduces antigen-specific sensitization and subsequent allergic reactions in a mouse model. Part Fibre Toxicol 2015, 12:20.

17. Cabanas A, Darr JA, E. L, Poliakoff M: A continous and clean one-step synthesis of nanoparticulate $\mathrm{Ce}_{1-\mathrm{x}} \mathrm{Zr}_{x} \mathrm{O}_{2}$ solid solutions in near-critical water. Chem Comm 2000, 2000:901-902.

18. Liu J, Römer I, Tang SVY, Valsami-Jones E, Palmer RE: Crystallinity depends on choice of iron salt precursor in the continous hydrothermal synthesis of Fe-Co oxide nanoparticles. RSC Adv 2017, 7:37436-37440.

19. He W, Liu Y, Wamer WG, Yin JJ: Electron spin resonance spectroscopy for the study of nanomaterial-mediated generation of reactive oxygen species. J Food Drug Anal 2014, 22:49-63.

20. Giannakou C, Aimonen K, Bloois LV, Catalan J, Geertsma RE, Gremmer ER, de Jong WH, Keizers $\mathrm{PH}$, Schwillens PL, Vandebriel RJ, Park MV: Sensitive method for endotoxin determination in nanomedicinal product samples. Nanomedicine (Lond) 2019, 14:1231-1246.

21. Harkema JR, Hotchkiss JA: In vivo effects of endotoxin on intraepithelial mucosubstances in rat pulmonary airways. Quantitative histochemistry. Am J Pathol 1992, 141:307-317.

22. Malyala P, Singh M: Endotoxin limits in formulations for preclinical research. J Pharm Sci 2008, 97:2041-2044.

23. Carragher DM, Rangel-Moreno J, Randall TD: Ectopic lymphoid tissues and local immunity. Semin Immunol 2008, 20:26-42.

24. Pitzalis C, Jones GW, Bombardieri M, Jones SA: Ectopic lymphoid-like structures in infection, cancer and autoimmunity. Nat Rev Immunol 2014, 14:447-462.

25. Meldrum K, Robertson SB, Romer I, Marczylo T, Dean LSN, Rogers A, Gant TW, Smith R, Tetley TD, Leonard MO: Cerium dioxide nanoparticles exacerbate house dust mite induced type II airway inflammation. Part Fibre Toxicol 2018, 15:24.

26. Schanen BC, Das S, Reilly CM, Warren WL, Self WT, Seal S, Drake DR, 3rd: Immunomodulation and Thelper $\mathrm{TH}(1) / \mathrm{TH}(2)$ response polarization by $\mathrm{CeO}(2)$ and $\mathrm{TiO}(2)$ nanoparticles. PLoS One 2013, 8:e62816.

27. Cho WS, Dart K, Nowakowska DJ, Zheng X, Donaldson K, Howie SE: Adjuvanticity and toxicity of cobalt oxide nanoparticles as an alternative vaccine adjuvant. Nanomedicine (Lond) 2012, 7:1495-1505.

28. de Haar C, Hassing I, Bol M, Bleumink R, Pieters R: Ultrafine but not fine particulate matter causes airway inflammation and allergic airway sensitization to co-administered antigen in mice. Clin Exp Allergy 2006, 36:1469-1479.

29. Randall TD: Bronchus-associated Iymphoid tissue (BALT) structure and function. Adv Immunol 2010, 107:187-241.

30. Hirahara K, Shinoda K, Endo Y, Ichikawa T, Nakayama T: Maintenance of memory-type pathogenic Th2 cells in the pathophysiology of chronic airway inflammation. Inflamm Regen 2018, 38:10. 
31. Hwang JY, Randall TD, Silva-Sanchez A: Inducible Bronchus-Associated Lymphoid Tissue: Taming Inflammation in the Lung. Front Immunol 2016, 7:258.

32. Cho WS, Duffin R, Bradley M, Megson IL, Macnee W, Howie SE, Donaldson K: NiO and Co304 nanoparticles induce lung DTH-like responses and alveolar lipoproteinosis. Eur Respir J 2012, 39:546-557.

33. Guest IC, Sell S: Bronchial lesions of mouse model of asthma are preceded by immune complex vasculitis and induced bronchial associated lymphoid tissue (iBALT). Lab Invest 2015, 95:886902.

34. Bates MA, Brandenberger C, Langohr I, Kumagai K, Harkema JR, Holian A, Pestka JJ: Silica Triggers Inflammation and Ectopic Lymphoid Neogenesis in the Lungs in Parallel with Accelerated Onset of Systemic Autoimmunity and Glomerulonephritis in the Lupus-Prone NZBWF1 Mouse. PLOS One 2015, 10:e0125481.

35. Blum JL, Rosenblum LK, Grunig G, Beasley MB, Xiong JQ, Zelikoff JT: Short-term inhalation of cadmium oxide nanoparticles alters pulmonary dynamics associated with lung injury, inflammation, and repair in a mouse model. Inhal Toxicol 2014, 26:48-58.

36. Hsieh SF, Bello D, Schmidt DF, Pal AK, Stella A, Isaacs JA, Rogers EJ: Mapping the biological oxidative damage of engineered nanomaterials. Small 2013, 9:1853-1865.

37. Ahsan SM, Rao CM, Ahmad MF: Nanoparticle-Protein Interaction: The Significance and Role of Protein Corona. Adv Exp Med Biol 2018, 1048:175-198.

38. Barbero F, Russo L, Vitali M, Piella J, Salvo I, Borrajo ML, Busquets-Fite M, Grandori R, Bastus NG, Casals E, Puntes V: Formation of the Protein Corona: The Interface between Nanoparticles and the Immune System. Semin Immunol 2017, 34:52-60.

39. Saptarshi SR, Duschl A, Lopata AL: Interaction of nanoparticles with proteins: relation to bioreactivity of the nanoparticle. J Nanobiotechnology 2013, 11:26.

40. Shannahan JH, Brown JM: Engineered nanomaterial exposure and the risk of allergic disease. Curr Opin Allergy Clin Immunol 2014, 14:95-99.

41. Han H, Park YH, Park HJ, Lee K, Um K, Park JW, Lee JH: Toxic and adjuvant effects of silica nanoparticles on ovalbumin-induced allergic airway inflammation in mice. Respir Res 2016, 17:60.

42. Horie M, Stowe M, Tabei M, Kuroda E: Pharyngeal aspiration of metal oxide nanoparticles showed potential of allergy aggravation effect to inhaled ovalbumin. Inhal Toxicol 2015, 27:181190. 


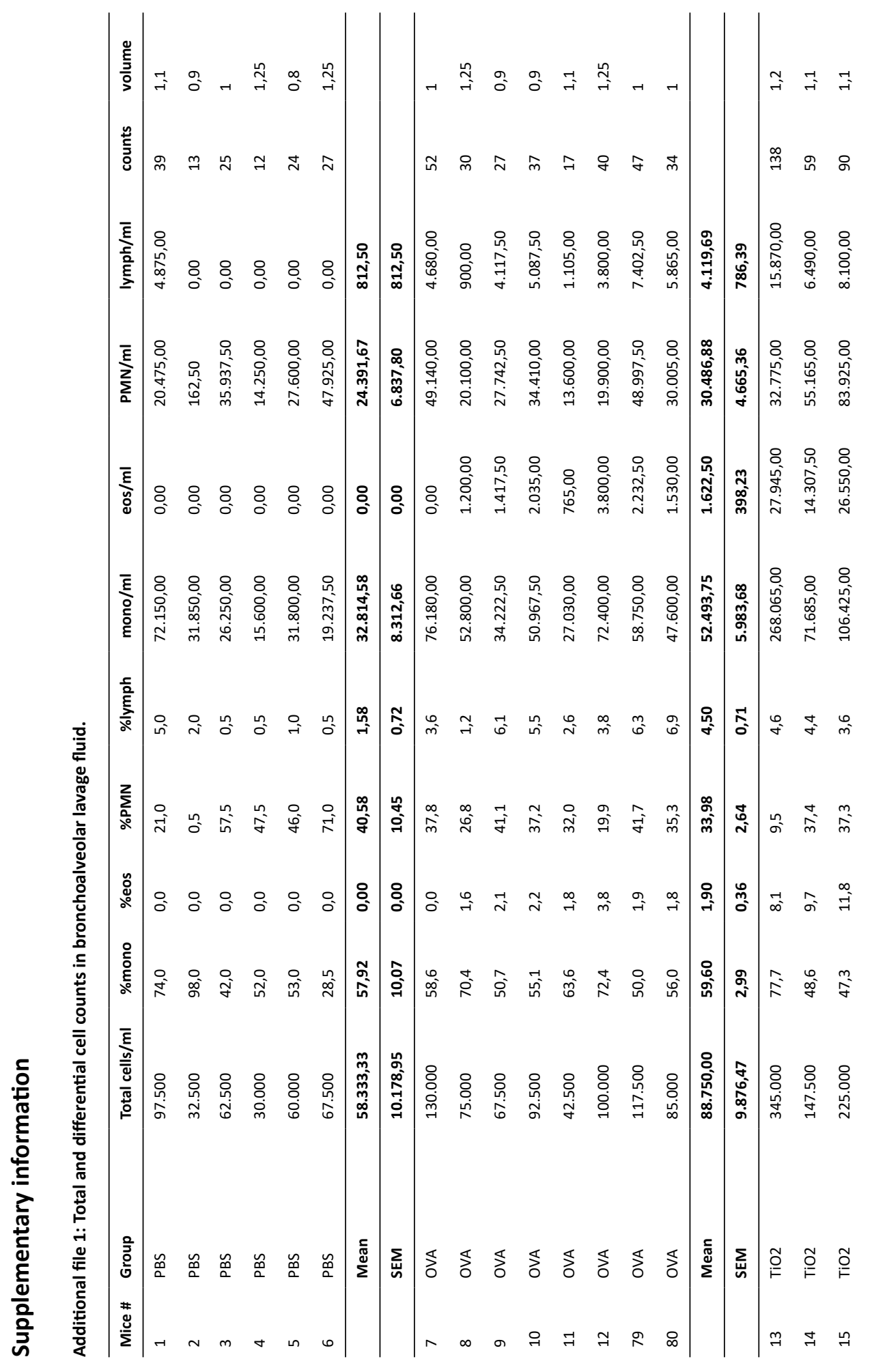




\begin{tabular}{|c|c|c|c|c|c|c|c|c|c|c|c|c|c|c|c|c|c|c|c|c|c|c|c|c|}
\hline$\vec{F}$ & $\underset{\sim}{\stackrel{N}{*}}$ & $\overrightarrow{-}$ & & & & $\vec{\Rightarrow}$ & $\rightarrow$ & $\stackrel{\stackrel{N}{-}}{\rightarrow}$ & $-r$ & & & & $\stackrel{\infty}{0_{0}^{+}}$ & $\stackrel{\infty}{0} \stackrel{0}{0}^{-}$ & $\rightarrow$ & $\stackrel{m}{\rightarrow}$ & $\underset{\sim}{\sim}$ & 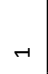 & & & & $\underset{\sim}{\stackrel{\sim}{\sim}}$ & $\stackrel{\stackrel{n}{-}}{\sim}$ & \\
\hline 8 & $\stackrel{\infty}{\curvearrowright}$ & $\infty$ & & & $\stackrel{m}{q}$ & $\stackrel{\text { I }}{ }$ & 8) & g & $\infty$ & 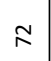 & & & 总 & $\hat{m}$ & $\underset{\exists}{J}$ & $\vec{\infty}$ & $\approx$ & $\ddot{\theta}$ & & & & f & 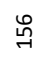 & $\stackrel{\infty}{\sim}$ \\
\hline & 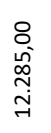 & $\begin{array}{l}\text { : } \\
\text { o. } \\
\text { on }\end{array}$ & 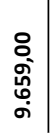 & 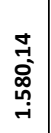 & 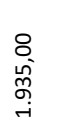 & $\begin{array}{l}\stackrel{8}{0} \\
\stackrel{n}{n} \\
\stackrel{n}{n}\end{array}$ & $\begin{array}{l}8 \\
\dot{5} \\
\stackrel{1}{7} \\
\vec{n}\end{array}$ & $\begin{array}{l}\stackrel{8}{\circ} \\
\text { ò } \\
\text { ஸ் }\end{array}$ & 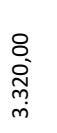 & $\begin{array}{l}8 \\
: \\
\dot{0} \\
0 \\
\dot{+}\end{array}$ & \begin{tabular}{l}
8 \\
\multirow{0}{0}{} \\
$\stackrel{0}{+}$ \\
$\dot{+}$
\end{tabular} & 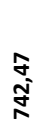 & 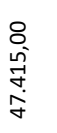 & 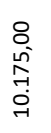 & 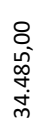 & 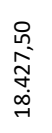 & 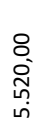 & $\begin{array}{l}8 \\
0 \\
0 \\
\qquad \\
\sigma \\
\sigma\end{array}$ & 依 & 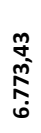 & $\begin{array}{l}8 \\
: \\
0 \\
0 \\
0 \\
0\end{array}$ & 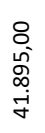 & 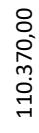 & $\begin{array}{l}8 \\
\stackrel{8}{0} \\
\text { m. } \\
\dot{0}\end{array}$ \\
\hline 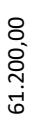 & 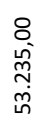 & \begin{tabular}{l} 
on \\
\multirow{1}{*}{} \\
$\underset{i}{0}$
\end{tabular} & 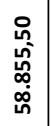 & 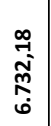 & 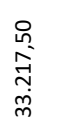 & 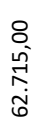 & 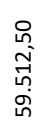 & 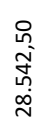 & $\begin{array}{l}8 \\
0 \\
0 \\
10 \\
0 \\
0 \\
\infty\end{array}$ & $\begin{array}{l}8 \\
0 \\
0 \\
\stackrel{0}{+} \\
\stackrel{+}{\wedge}\end{array}$ & $\begin{array}{l}\text { గू. } \\
\text { న్ర్๋ } \\
\text { ஸे }\end{array}$ & $\begin{array}{l}\hat{\alpha} \\
\infty \\
\hat{\infty}^{-} \\
\infty \\
\infty\end{array}$ & $\begin{array}{l}\stackrel{8}{0} \\
\stackrel{.}{0} \\
m \\
\infty \\
\infty\end{array}$ & 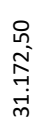 & $\begin{array}{l}8 \\
0 \\
0 \\
\overrightarrow{7} \\
\dot{0}\end{array}$ & 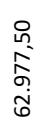 & $\begin{array}{l}8 \\
0 \\
\circ \\
\circ \\
\sigma\end{array}$ & 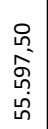 & 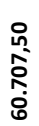 & 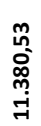 & 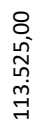 & $\begin{array}{l}8 \\
0 \\
0 \\
6 \\
0 \\
\infty\end{array}$ & 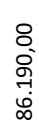 & 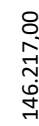 \\
\hline $\begin{array}{l}8 \\
\text { ò } \\
\text { on. } \\
\text { o. }\end{array}$ & 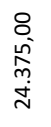 & 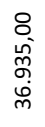 & 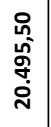 & $\begin{array}{l}\vec{m} \\
\hat{o} \\
\stackrel{\circ}{0} \\
\dot{+}\end{array}$ & 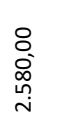 & $\begin{array}{l}8 \\
: \\
0 \\
0 \\
0\end{array}$ & 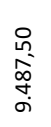 & $\begin{array}{l}8 \\
\circ \\
\text { స్ } \\
\text { r. }\end{array}$ & 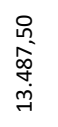 & 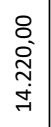 & ֻ. & 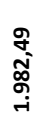 & 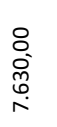 & 足 & 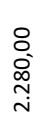 & : & $\begin{array}{l}8 \\
\text { ¿े. } \\
\stackrel{8}{\sigma}\end{array}$ & \begin{tabular}{l}
$\stackrel{0}{n}$ \\
$\underset{\sim}{*}$ \\
\multirow{f}{*}{}
\end{tabular} & 员 & $\begin{array}{l}\text { o. } \\
\stackrel{\text { Dें }}{\text { Tे }}\end{array}$ & $\begin{array}{l}8 \\
\stackrel{8}{\stackrel{N}{7}} \\
\stackrel{7}{N}\end{array}$ & $\begin{array}{l}8 \\
0 \\
\infty \\
\infty \\
\infty \\
i n\end{array}$ & 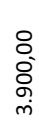 & 8 \\
\hline 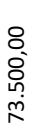 & 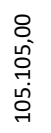 & 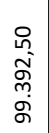 & 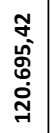 & $\begin{array}{l}\tilde{N} \\
\stackrel{n}{2} \\
\hat{m} \\
\stackrel{m}{m} \\
\end{array}$ & $\begin{array}{l}0 \\
0 \\
\stackrel{0}{0} \\
0 \\
0 \\
0\end{array}$ & $\begin{array}{l}8 \\
0 \\
0 \\
+ \\
0 \\
0 \\
0 \\
0\end{array}$ & 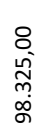 & 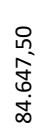 & 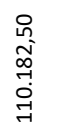 & $\begin{array}{l}8 \\
0 \\
0 \\
0 \\
\dot{0} \\
\infty\end{array}$ & 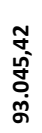 & 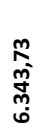 & 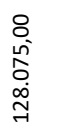 & 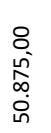 & 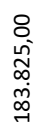 & 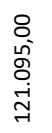 & $\begin{array}{l}8 \\
0 \\
0 \\
0 \\
\dot{y}\end{array}$ & 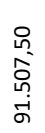 & 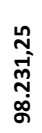 & $\begin{array}{l}\vec{a} \\
\text { s. } \\
\text { กิ } \\
\text { dे }\end{array}$ & 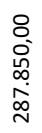 & 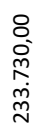 & 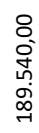 & 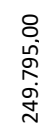 \\
\hline$\hat{m}$ & $\tilde{m}^{-}$ & $\stackrel{\infty}{\underset{\sim}{*}}$ & 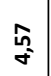 & $\underset{0}{q}$ & $\underset{\rightarrow}{\infty}$ & $\stackrel{\sigma}{m}$ & $\stackrel{\circ}{m}$ & $\stackrel{+}{\leftarrow}$ & $\stackrel{6}{-i}$ & $\underset{i}{0}$ & $\stackrel{\infty}{\stackrel{\infty}{\sim}}$ & $\stackrel{f}{0}$ & $\stackrel{ \pm}{\stackrel{ \pm}{二}}$ & $\stackrel{0}{g}$ & $\underset{\vec{y}}{\vec{y}}$ & $\vec{a}$ & $\underset{\sim}{\stackrel{+}{\sim}}$ & $\overbrace{0}^{\circ}$ & $\underset{\substack{N \\
N}}{ }$ & $\stackrel{\circ}{\stackrel{\leftrightarrow}{-}}$ & 号 & $\underset{\exists}{\Xi}$ & $\stackrel{m}{\infty}$ & $\stackrel{+}{\vec{m}}$ \\
\hline$\stackrel{\infty}{\infty} \stackrel{\infty}{q}^{-}$ & $\stackrel{m}{\sim}$ & 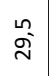 & 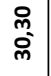 & 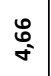 & $\begin{array}{l}\stackrel{\rho}{\rho} \\
\text { ले }\end{array}$ & 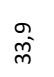 & 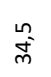 & 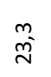 & $\begin{array}{l}\infty \\
\infty_{m}^{\infty} \\
m^{2}\end{array}$ & $\begin{array}{l}\mathfrak{F} \\
F\end{array}$ & 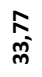 & $\stackrel{n}{n}$ & 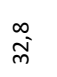 & $\hat{m}^{\bar{m}}$ & 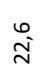 & $\vec{m}$ & $\stackrel{\stackrel{N}{m}}{\tilde{m}^{2}}$ & w్ & 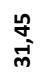 & $\stackrel{\leftrightarrow}{\underset{T}{-}}$ & $\stackrel{\tilde{\gamma}}{\tilde{N}}$ & $\stackrel{+}{\stackrel{N}{N}}$ & $\overrightarrow{\mathbb{N}}$ & 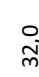 \\
\hline$\tilde{\sigma}$ & 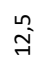 & $\stackrel{\infty}{\stackrel{-}{二}}$ & $\begin{array}{l}\tilde{o} \\
\exists \\
\exists\end{array}$ & 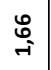 & $\stackrel{\pi}{N}$ & $\stackrel{0}{m_{m}^{-}}$ & $\stackrel{\text { Ln }}{\text { ஸn }^{\prime}}$ & $\stackrel{N}{m}$ & 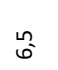 & $\stackrel{\text { ㄱ. }}{=}$ & $\stackrel{\substack{\infty \\
\leftarrow}}{\leftarrow}$ & $\begin{array}{c}\text { of } \\
\text { o }\end{array}$ & $\stackrel{\infty}{i}$ & $\stackrel{m}{o}$ & 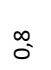 & : & $\underset{-}{0}$ & $\stackrel{m}{o}$ & ลิ & $\stackrel{m}{0}$ & $\stackrel{\leftrightarrow}{\rightarrow}$ & $\underset{\sim}{\sigma}$ & $\stackrel{\rightarrow}{\mathrm{i}}$ & 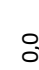 \\
\hline 晏 & ભે & $\stackrel{g}{\approx}$ & $\begin{array}{l}\hat{o} \\
\dot{f}\end{array}$ & 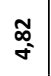 & $\begin{array}{l}\text { a } \\
\dot{0}\end{array}$ & $\begin{array}{l}0 \\
\infty_{n}^{\infty}\end{array}$ & 㝕 & تิ & $\overrightarrow{\tilde{N}}$ & $\stackrel{m}{\infty_{d}^{\infty}}$ & 㔛 & $\underset{m}{\stackrel{9}{m}}$ & $\begin{array}{l}\stackrel{0}{r} \\
\text { r }\end{array}$ & 号 & 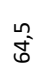 & $\begin{array}{l}\infty \\
\text { Oे }\end{array}$ & $\hat{g}$ & $\stackrel{\overrightarrow{0}}{\infty_{n}^{-}}$ & 离 & สุ & $\begin{array}{l}0 \\
: \\
0\end{array}$ & $\begin{array}{l}0 \\
\tilde{\theta} \\
\tilde{0}\end{array}$ & $\begin{array}{l}0 \\
\stackrel{0}{\sigma}\end{array}$ & $\begin{array}{l}0 \\
\text { [f }\end{array}$ \\
\hline 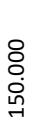 & $\begin{array}{l}\text { ષ } \\
\text { ஸે }\end{array}$ & 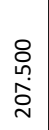 & 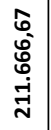 & 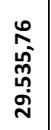 & 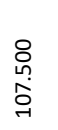 & $\begin{array}{l}\stackrel{8}{0} \\
\stackrel{\infty}{\infty} \\
\stackrel{\infty}{\rightarrow-1}\end{array}$ & 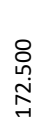 & $\begin{array}{l}\stackrel{8}{0} \\
\stackrel{\text { I }}{7}\end{array}$ & $\begin{array}{l}\stackrel{\circ}{\leftrightarrow} \\
\stackrel{n}{0}\end{array}$ & 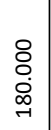 & 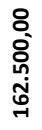 & 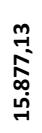 & $\underset{\substack{\stackrel{0}{N} \\
\text { N }}}{ }$ & $\begin{array}{l}\stackrel{8}{0} \\
\text { న్ }\end{array}$ & 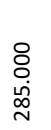 & $\begin{array}{l}\stackrel{8}{0} \\
\text { î̀ } \\
\text { مे }\end{array}$ & $\begin{array}{l}\text { o } \\
\dot{m}\end{array}$ & 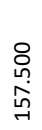 & 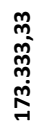 & \begin{tabular}{l}
$\hat{0}$ \\
\multirow{I}{|}{} \\
$\dot{j}$ \\
$\dot{y}$
\end{tabular} & 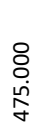 & 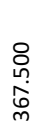 & 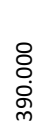 & $\begin{array}{l}\stackrel{8}{\circ} \\
\stackrel{h}{1} \\
\text { f }\end{array}$ \\
\hline 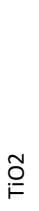 & 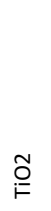 & $\stackrel{\widetilde{O}}{\circ}$ & $\begin{array}{l}\text { एٓ } \\
\stackrel{\tilde{\varpi}}{\Sigma}\end{array}$ & $\sum_{\text {出 }}$ & 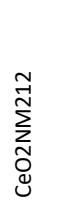 & 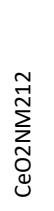 & 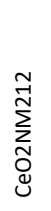 & 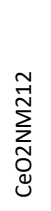 & 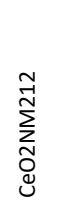 & 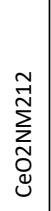 & $\begin{array}{l}\text { 胥 } \\
\sum_{\Sigma}\end{array}$ & $\sum_{\mathrm{u}}$ & 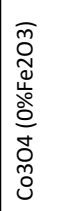 & 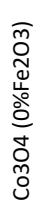 & 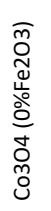 & 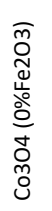 & 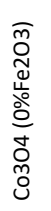 & 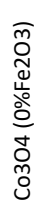 & $\begin{array}{l}\text { 胥 } \\
\sum^{\circ}\end{array}$ & $\sum_{\text {U }}$ & 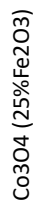 & 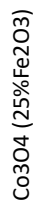 & 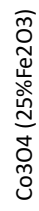 & 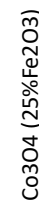 \\
\hline & $r$ & 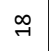 & & & ने & ㅇ & $\vec{\sim}$ & $\approx$ & $\stackrel{m}{\sim}$ & & & & $\stackrel{\sim}{\sim}$ & $\stackrel{\mathscr{N}}{\sim}$ & $\hat{\sim}$ & $\stackrel{\infty}{\sim}$ & 尺ి & & & & $\vec{m}$ & $\tilde{m}$ & $\stackrel{m}{m}$ & I \\
\hline
\end{tabular}




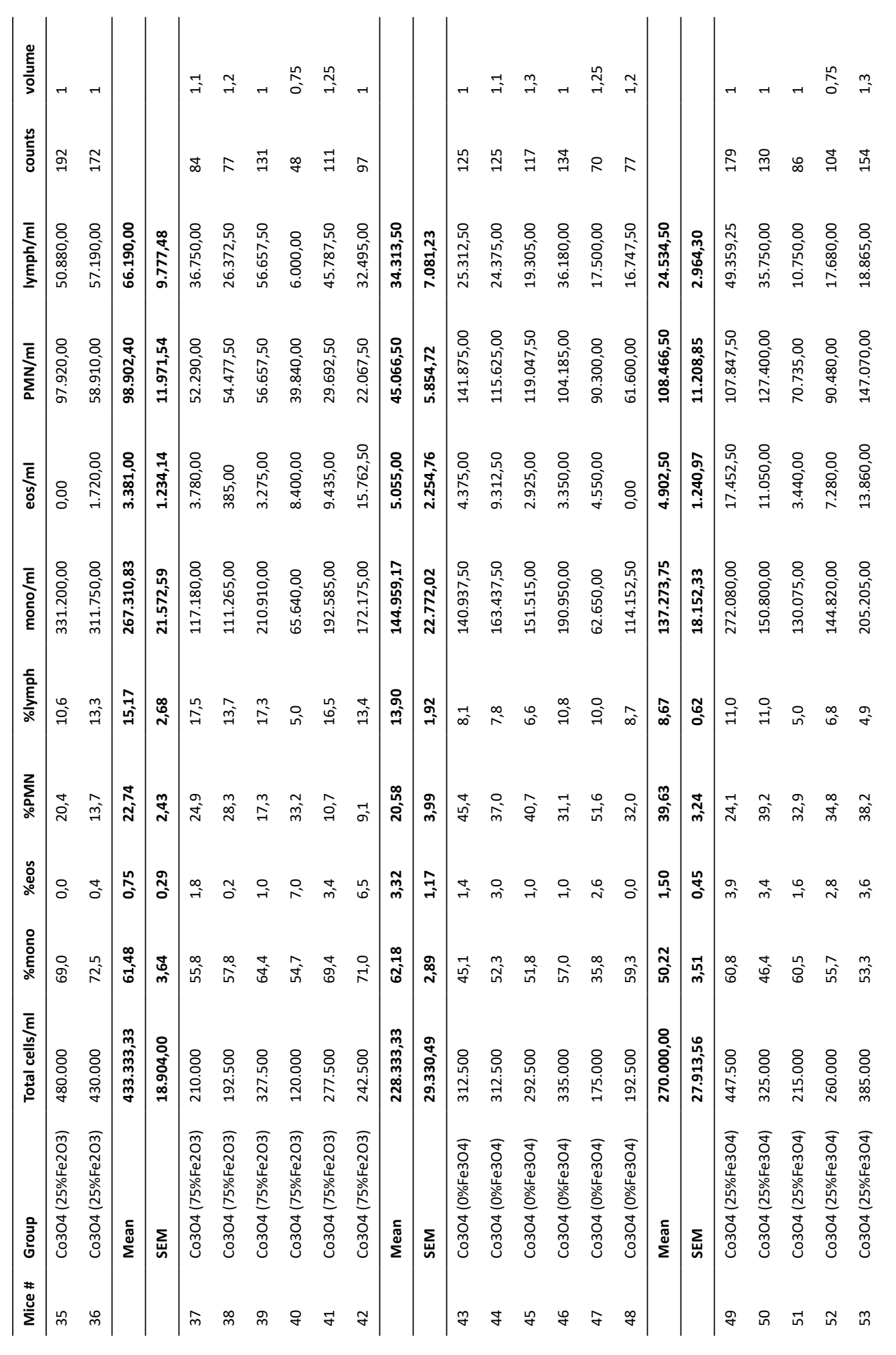




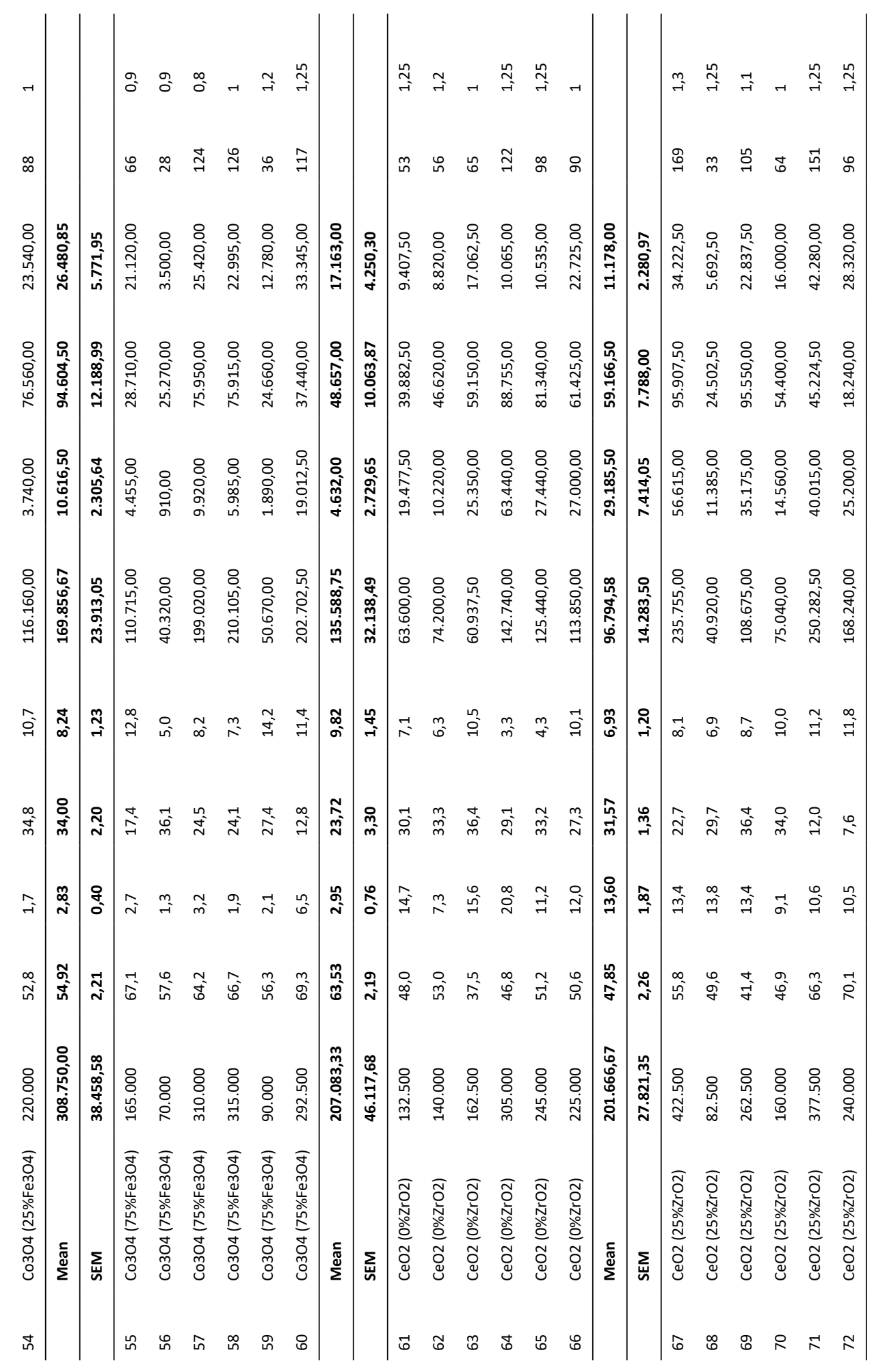




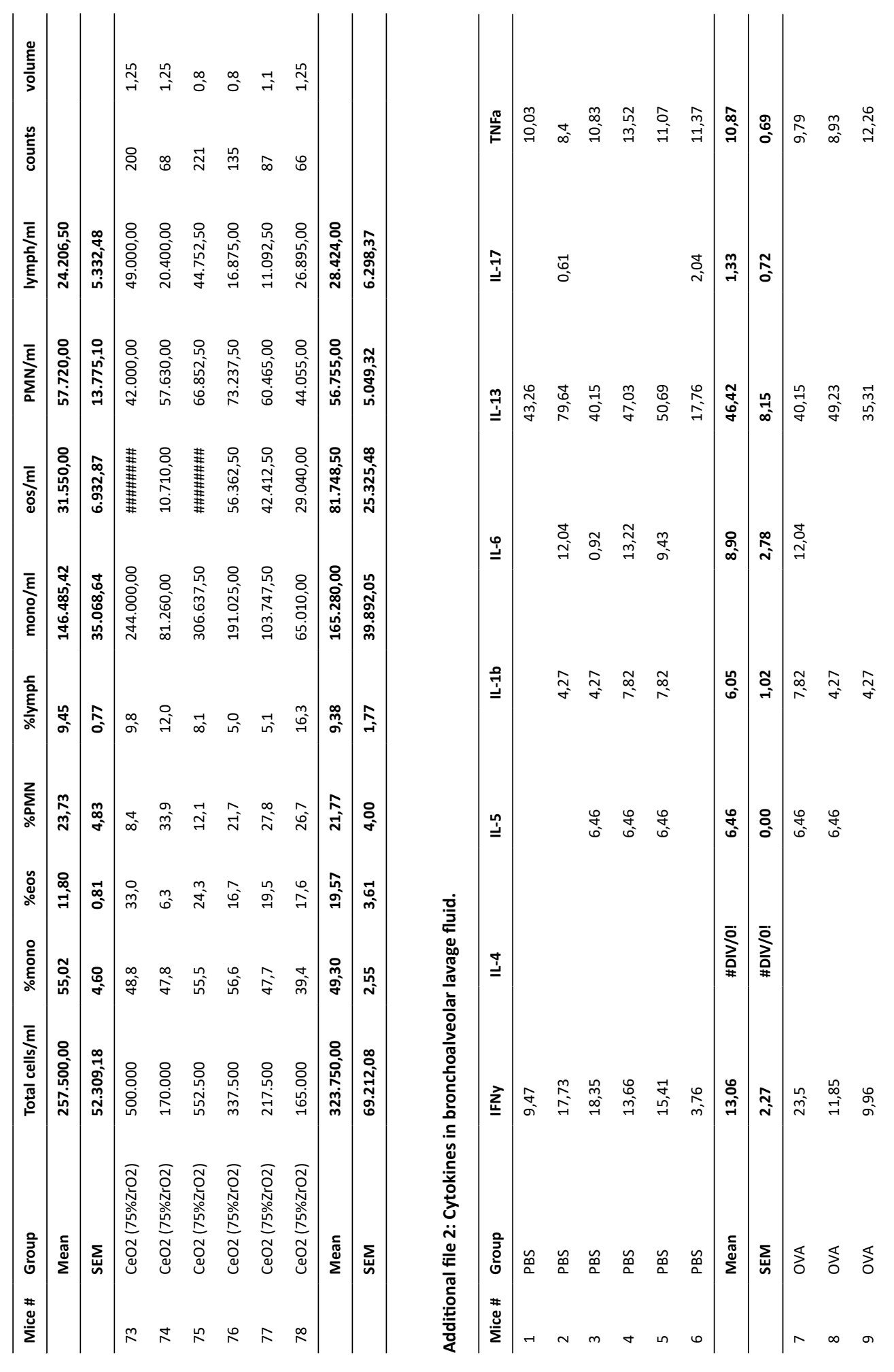




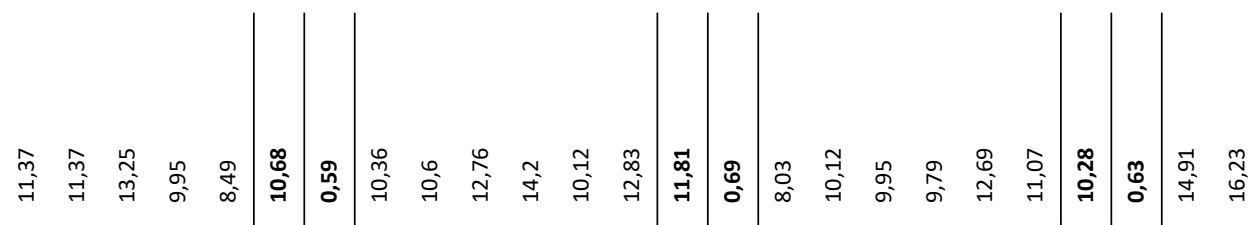

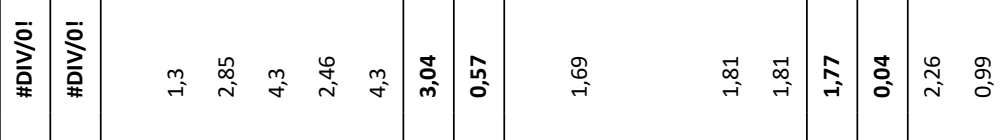

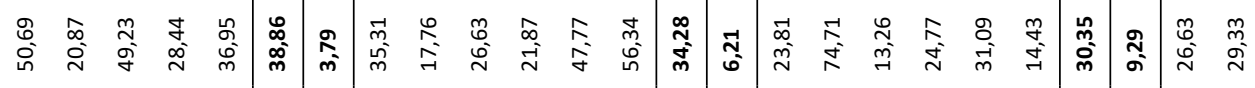

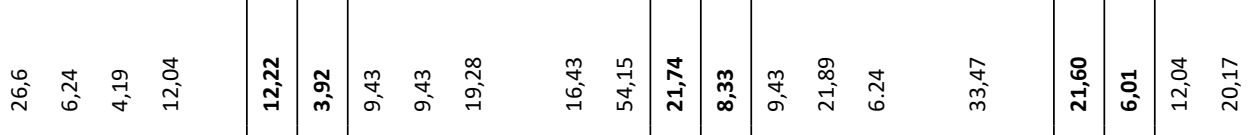

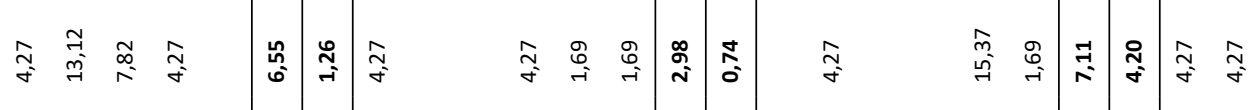

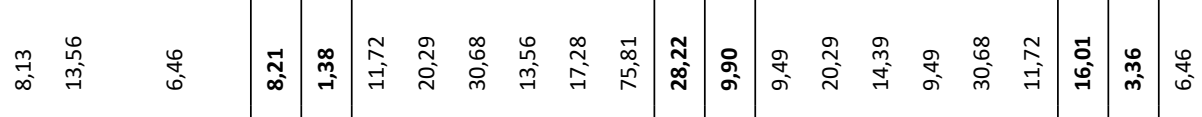

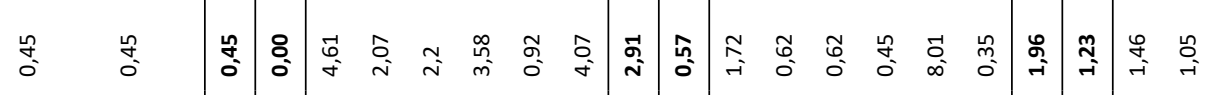

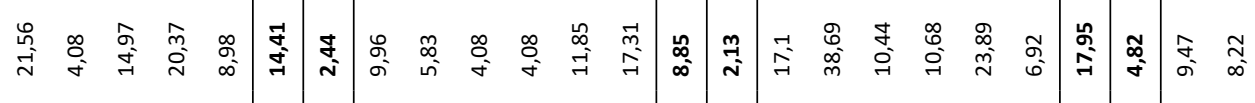

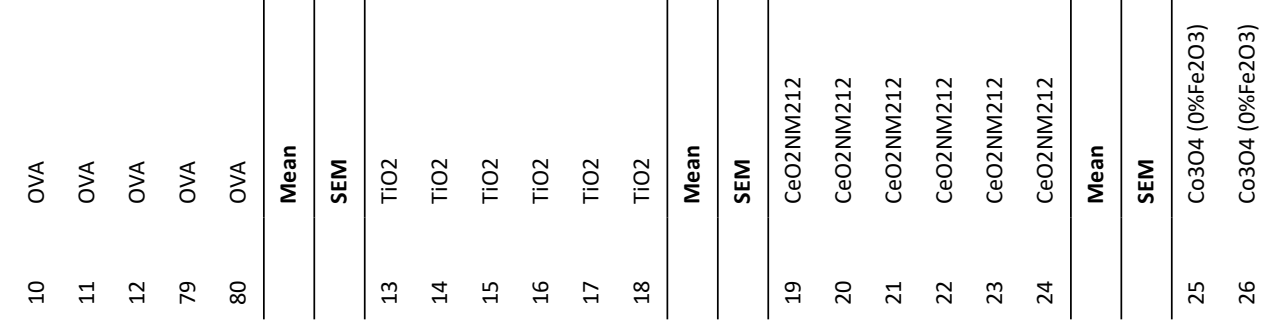




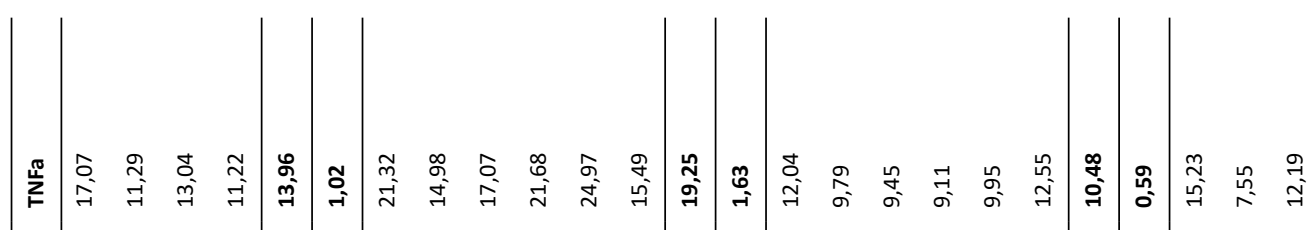

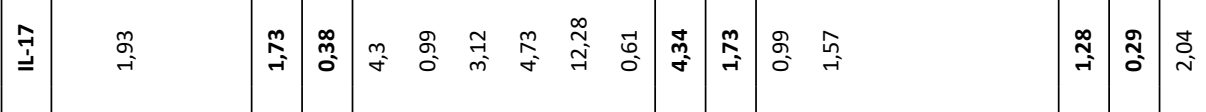

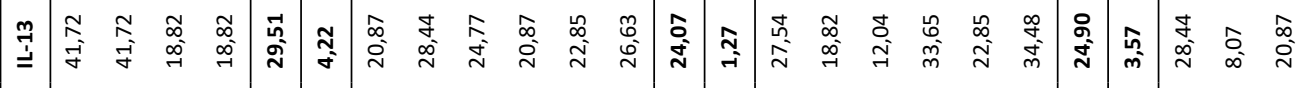

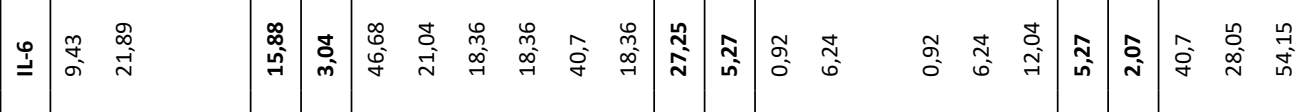

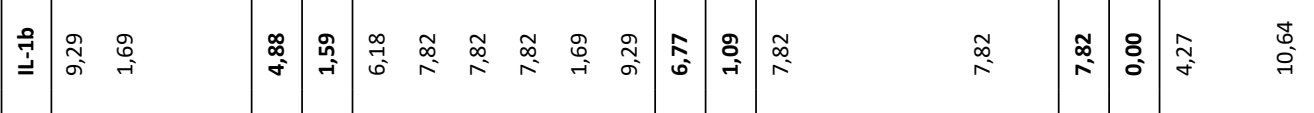

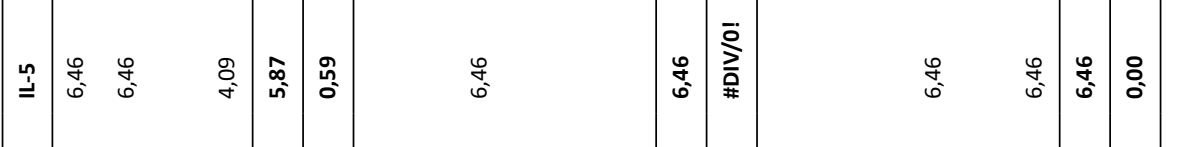

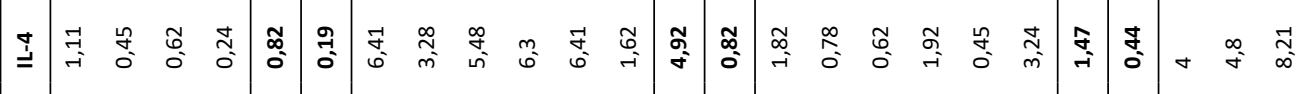

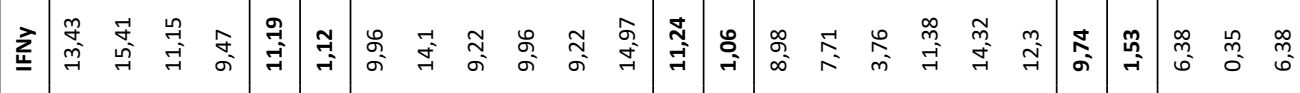

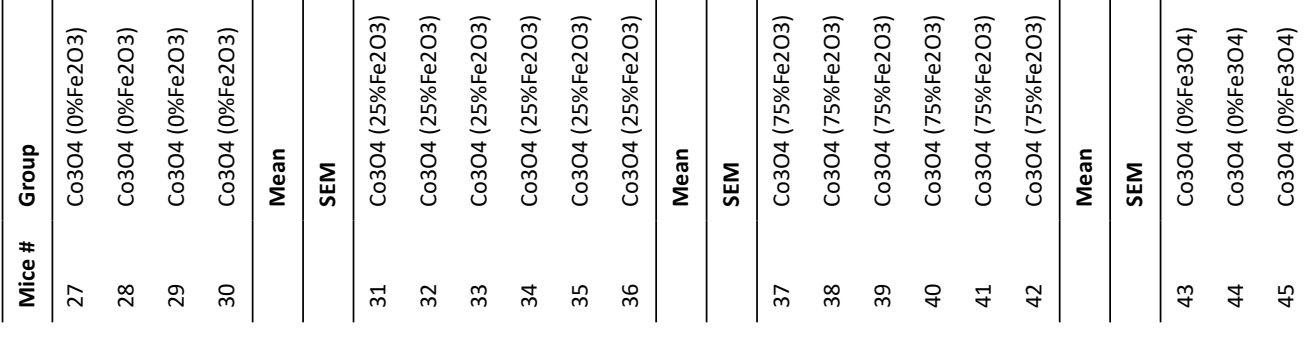




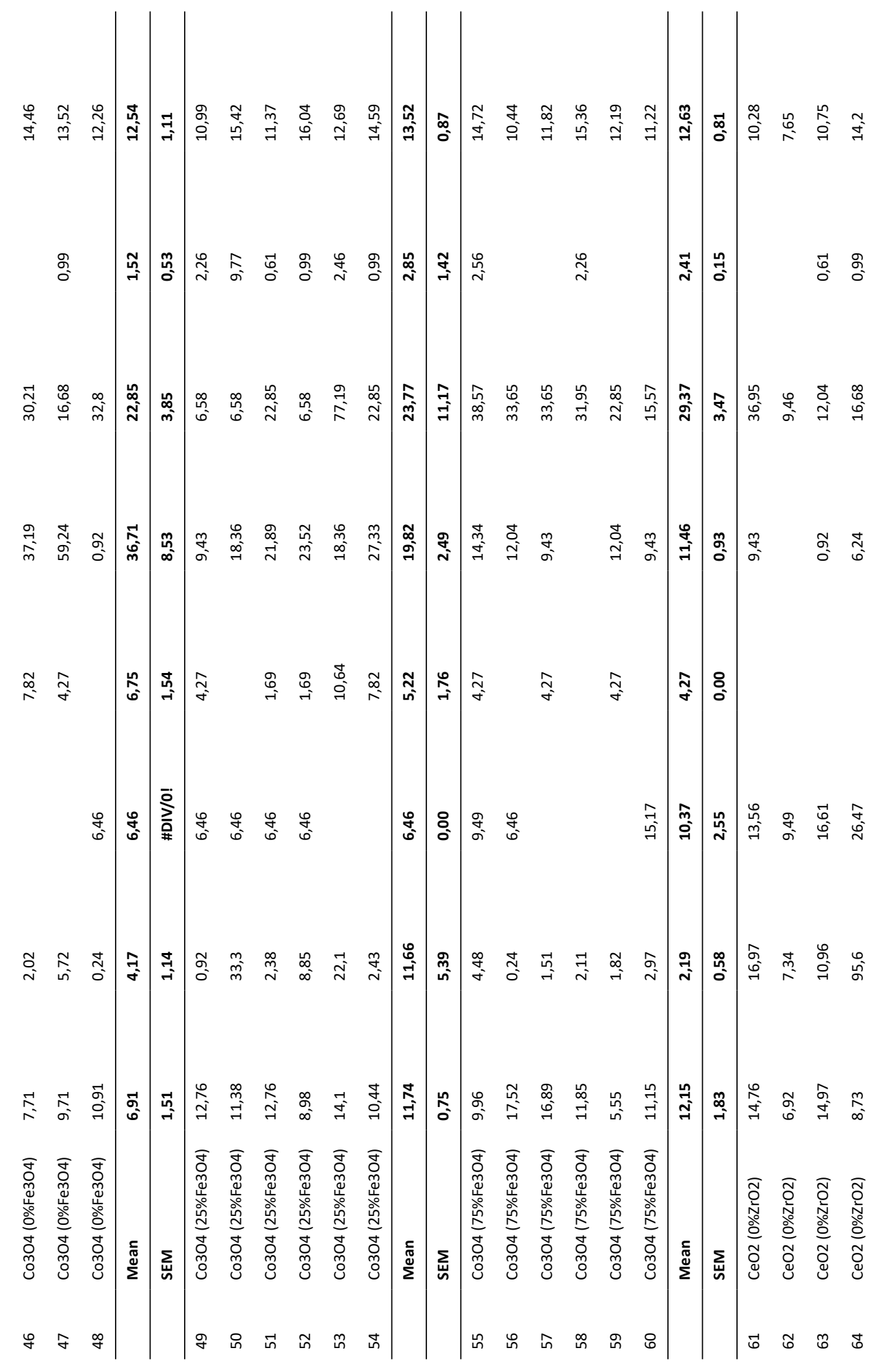




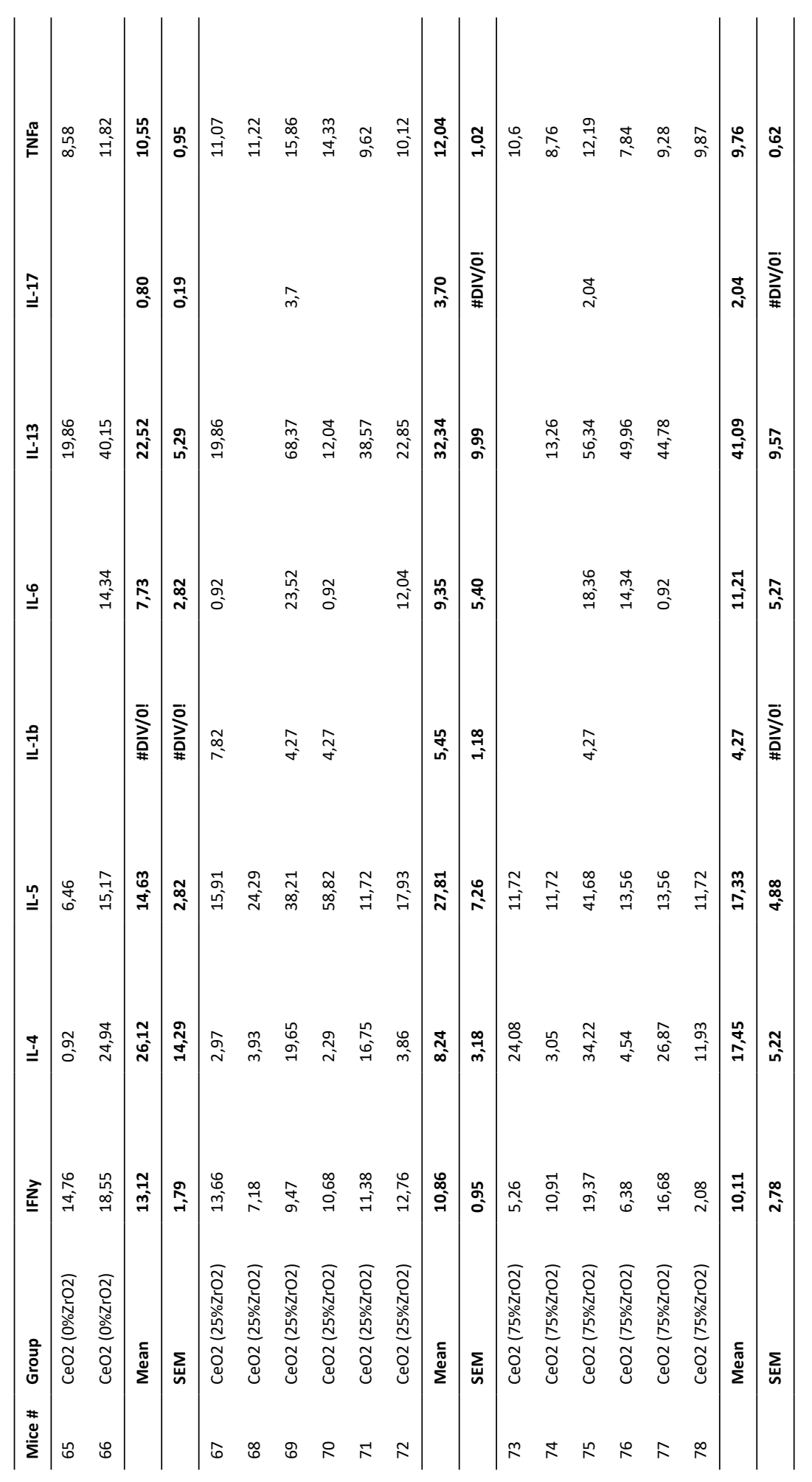




\begin{tabular}{|c|c|c|c|c|c|c|c|c|c|c|c|}
\hline & \multirow[b]{2}{*}{ OVA } & \multicolumn{3}{|c|}{$\mathrm{CeO}_{2}(\mathrm{x} \% \mathrm{Zr})$} & \multicolumn{3}{|c|}{$\mathrm{Co}_{3} \mathrm{O}_{4}\left(\mathrm{x} \% \mathrm{Fe}_{2} \mathrm{O}_{3}\right)$} & \multicolumn{3}{|c|}{$\mathrm{Co}_{3} \mathrm{O}_{4}\left(\mathrm{x} \% \mathrm{Fe}_{2} \mathrm{O}_{3}\right)$} & \multirow[b]{3}{*}{200} \\
\hline & & $0 \%$ & $27 \%$ & $78 \%$ & $0 \%$ & $25 \%$ & $75 \%$ & $0 \%$ & $25 \%$ & $75 \%$ & \\
\hline $\lg E$ & & & & & & & & & & & \\
\hline $\lg \mathbf{l g} 1$ & & & & & & & & & & & 30000 \\
\hline Total cell count BALF & & & & $>$ & & & & & & & 250000 \\
\hline$\%$ Neurtophils BALF & & & & & & & 2 & & & $->$ & 30 \\
\hline$\%$ Eosinophils BALF & & & & & & & & & & & 10 \\
\hline \% Lymphocytes BALF & & & & & & & & & & & 10 \\
\hline IL-4 in BALF & & & & & & & & & & & 15 \\
\hline IL-5 in BALF & & & & & & & & & - & $\Rightarrow$ & 15 \\
\hline IL-6 in BALF & & & & & & & & & & 8 & 20 \\
\hline IL-13 in BALF & & & & & & & & & & & 30 \\
\hline Mucosubstance G5 & & & & & & & $\Delta$ & & & $>$ & 2.5 \\
\hline Mucosubstance G11 & & & & & & & & & & & 2 \\
\hline Oesinophilic density & & & & & & & $P$ & & & & 0.7 \\
\hline B-lymphocyte density & & & & & & & & & & & 6 \\
\hline
\end{tabular}

Figure S1: The influence of the ability of NPs to induce superoxide generation as measured in an acellular EPR assay on the different biomarkers of effects.

The shading indicates the lowest (light grey) to highest (dark grey) response of an NP (relative to each other) for each of the biomarkers. The arrows indicate an increase $(\lambda)$ or decrease $(\checkmark)$ with decreasing amount of superoxide generation. 


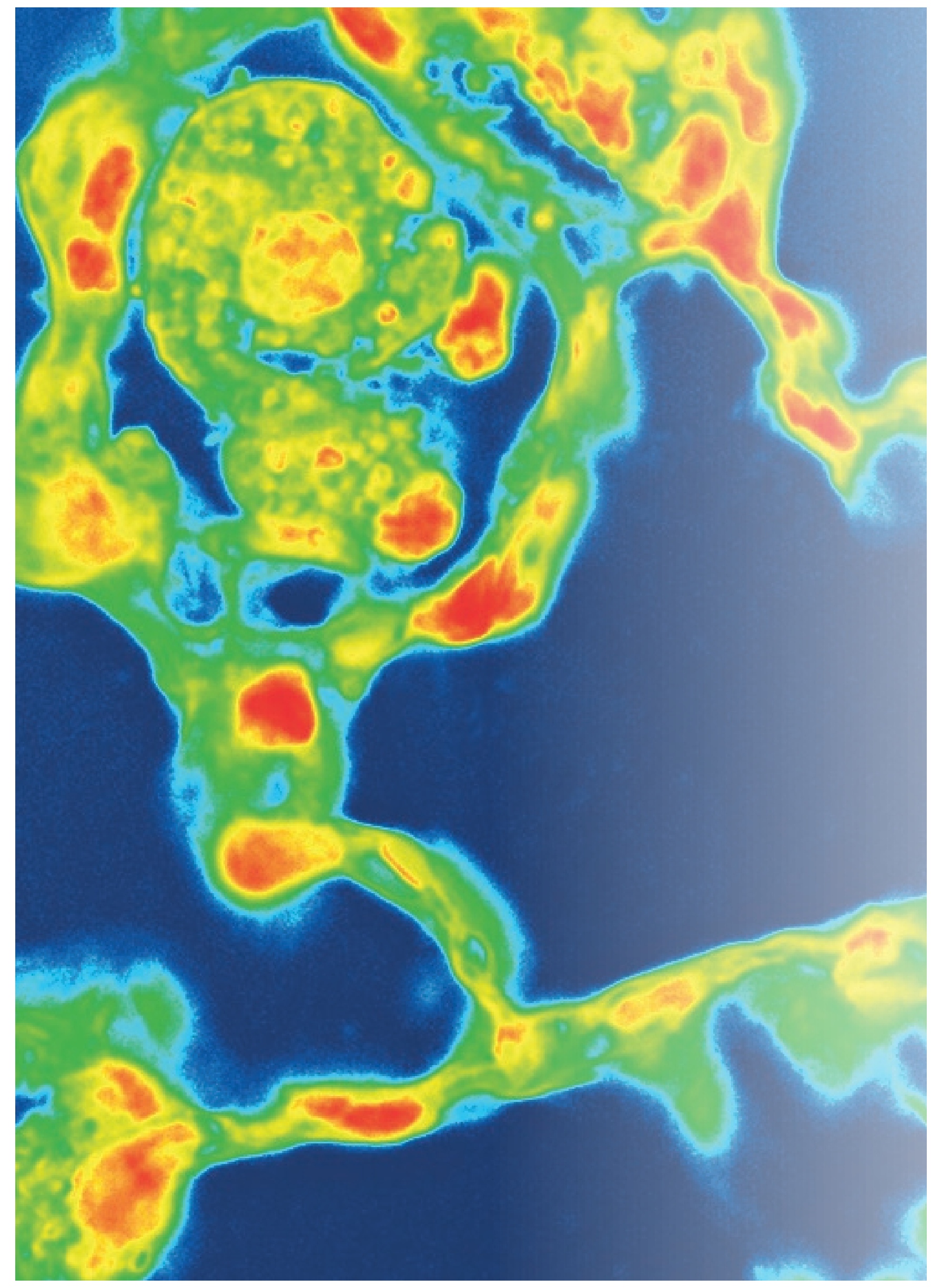




\section{Chapter 6}

\section{The influence of chemical}

composition and redox activity of nanomaterials on in vitro inflammasome activation and dendritic cell maturation

Susan Dekkers' ', Jolanda P. Vermeulen', Jasper G.D. Wigger', Geert van der Horst' ${ }^{1}$, Rory Verhagen', Jia Ning ', Isabella Römer ${ }^{2}$, Wim H. de Jong' ', Flemming R. Cassee ${ }^{1,3}$, Rob J. Vandebriel'

${ }^{1}$ National Institute for Public Health and the Environment (RIVM), Bilthoven, The Netherlands,

2 School of Geography, Earth and Environmental Sciences, University of Birmingham, Birmingham, United Kingdom,

${ }^{3}$ Institute for Risk Assessment Sciences, Utrecht University, The Netherlands 


\begin{abstract}
There is limited understanding on how physicochemical properties of nanoparticles (NPs) may influence their ability to enhance the induction of allergic responses. One of the proposed mechanisms of toxicity is the redox activity of the NPs. However, in our recent in vivo study on adjuvant properties of various NPs no relation was observed between the acellular redox activity of the NPs and the ability to enhance allergic airway sensitisation. Here we study the same $\mathrm{Zr}$-doped $\mathrm{CeO}_{2}$ and Fe-doped $\mathrm{CO}_{3} \mathrm{O}_{4}$ NPs in two different in vitro assays, inflammasome activation in macrophages and maturation of dendritic cells (DCs), to investigate whether the differences between the type of immune responses observed in vivo can be predicted using these in vitro assays. $\mathrm{CeO}_{2} \mathrm{NPs}$ (doped and undoped) but not $\mathrm{CO}_{3} \mathrm{O}_{4} \mathrm{NPs}$ (doped and undoped) induced inflammasome activation. Undoped $\mathrm{CO}_{3} \mathrm{O}_{4}$ NPs increased interleukine-12p40 (IL-12p40) production by DCs, while $\mathrm{CeO}_{2}$ NPs (doped and undoped) did not. Doping effects of $\mathrm{CO}_{3} \mathrm{O}_{4}$ NPs were mixed. The results of these in vitro assays indicate that the type of immune response of the NPs is primarily related to chemical composition and not to size or acellular redox activity, similar to our previous results in the in vivo study. Furthermore, a pronounced increase in IL-12p40 in DCs in vitro seemed to be indicative for lymphoid accumulation in lung tissue in vivo. However, additional studies with NPs with different chemical compositions and in vitro assays covering other mechanisms are needed to more accurately predict the adjuvant activity of NPs in vivo.
\end{abstract}




\section{Introduction}

Metal oxide nanoparticles (NPs) have been shown to be able to act as adjuvants ${ }^{[1-3]}$. In vaccines, this property is further investigated and can be beneficial ${ }^{[1]}$. However, the adjuvant properties of NPs may also have negative health effects, for example in the development of allergic airway diseases like asthma.

There is limited understanding on the physicochemical properties of NPs that influence their adjuvant properties ${ }^{[2]}$. From the use of NPs in vaccines, we know that NPs can induce adjuvant activities by enhancing antigen processing or by inducing and amplifying protective immunity, for example by inflammasome activation ${ }^{[4]}$. Increases in the maturation of dendritic cells (DCs) and proliferation and differentiation of T-cells have been observed after in vitro exposure to NPs and after in vivo co-exposure of an allergen and NPs during the sensitisation phase in mouse models for airway allergy ${ }^{[2]}$. Molecular interactions that drive these cellular responses have been suggested to involve increases in reactive oxygen species (ROS) caused by NPs with an oxidative surface chemistry, inflammasome activation, cellular injury and the induction of DC stimulating cytokines and chemokines by epithelial cells (either direct or via oxidative stress) ${ }^{[2]}$.

Physicochemical properties of metal and metal oxide NPs that have been postulated to drive these molecular interactions are amongst others a large surface area ${ }^{[5,6]}$, reactive surface chemistry (e.g. through Haber-Weiss and Fenton reaction mechanisms) [7], a conduction band energy (Ec) level that overlaps with the cellular redox potential ${ }^{[8]}$ ) and a fibre- or urchin-like shape ${ }^{[9,10]}$.

Results of a recent study on adjuvant properties of various NPs in a mouse model of airway hypersensitivity indicated that chemical composition of NPs influences the type of immune response, but no relation was found between the acellular redox activity of the NPs and their adjuvant properties ${ }^{[11]}$. To investigate the influence of chemical composition and redox activity, $\mathrm{Zr}$-doped $\mathrm{CeO}_{2} \mathrm{NPs}$ and Fe-doped $\mathrm{Co}_{3} \mathrm{O}_{4} \mathrm{NPs}$ were selected, as the ability to induce oxygen radicals was expected to be larger for $\mathrm{Co}_{3} \mathrm{O}_{4}$ compared to $\mathrm{CeO}_{2} \mathrm{NPs}$ based on their Ec levels, while $\mathrm{Zr}$ - and Fe- doping was expected to reduce the redox activity of $\mathrm{CeO}_{2}$ and $\mathrm{Co}_{3} \mathrm{O}_{4}$ NPs, respectively ${ }^{[11]}$. Here we investigate the same NPs in two different in vitro assays, to study whether the differences between the type of immune responses observed after exposure to $\mathrm{CeO}_{2}$ and $\mathrm{Co}_{3} \mathrm{O}_{4}$ NPs in vivo can be predicted using these in vitro assays. These two in vitro assays are inflammasome activation in macrophages and maturation of dendritic cells, as these mechanisms are known to play an important role in NP-enhanced induction of allergic airway responses ${ }^{[2,12]}$. Inflammasome activation has been

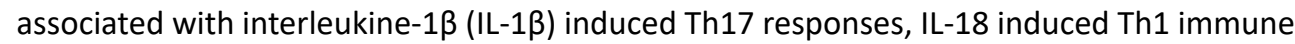
responses, and neutrophilic inflammation in vivo ${ }^{[13]}$. Inflammasome activation, IL-1ß and IL- 
18 have also been associated with Th2 immune responses, but these pathways are less well established ${ }^{[14-18]}$. DC maturation in vitro has been associated with $\mathrm{T}$ - and B-cell proliferation in vivo. Depending on the chemical composition, surface chemistry, shape and dose, NPs may promote DCs' capability to induce an IL-12 induced Th1 response, an IL-4 induced Th2 response and/or an IL- 6 induced Th17 response ${ }^{[12,19]}$. For inflammasome activation, phorbol 12-myristate 13-acetate (PMA) differentiated THP-1 macrophages were used as this well-studied, easy to handle cell type is a robust and reproducible model to investigate inflammasome activation ${ }^{[20]}$. Furthermore, NLRP3- and ASC-deficient THP-1 macrophages are commercially available, enabling a reliable way to control for the involvement of the NLRP3 inflammasome in the responses obtained ${ }^{[21,22]}$. For dendritic cell maturation, differentiated monocytes isolated from human peripheral blood mononuclear cells (PBMCs) were used as this in vitro model is generally accepted to measure effects on DC maturation and has been suggested as a promising in vitro screen predictive for adjuvant activity of NPs in vivo ${ }^{[3]}$.

Our hypothesis is that the influence of chemical composition and doping of NPs on the type of immune response underlying the adjuvant activity of NPs in vivo can be predicted using in vitro inflammasome activation and DC maturation assays.

\section{Materials and Methods}

\subsection{NP production and characterization}

All NPs were produced using supercritical water hydrothermal synthesis ${ }^{[23,24]}$. The production and characterization of the NPs has been described in our previous publication ${ }^{[11]}$. The main characteristics are summarised in Table 1. Zr-doping of the $\mathrm{CeO}_{2}$ NPs did not increase the primary or gravimetric particle size, but did increase the hydrodynamic size in water as measured with Dynamic Light Scattering (DLS). Fe-doping of the $\mathrm{Co}_{3} \mathrm{O}_{4} \mathrm{NPs}$ reduced the primary particle size and increased the gravimetric size for the $\mathrm{Co}_{3} \mathrm{O}_{4} \mathrm{NPs}$, except for the $\mathrm{Co}_{3} \mathrm{O}_{4}\left(75 \% \mathrm{Fe}_{2} \mathrm{O}_{3}\right)$ NPs. Fe-doping with $\mathrm{Fe}_{3} \mathrm{O}_{4}$ as a precursor increased the hydrodynamic particle size of the $\mathrm{Co}_{3} \mathrm{O}_{4}\left(25 \%\right.$ and $\left.75 \% \mathrm{Fe}_{3} \mathrm{O}_{4}\right)$ NPs, however, this increase was larger with $25 \%$, than with $75 \%$ Fe-doping (see Table 1). Electron Paramagnetic Resonance (EPR) analysis using tempone- $\mathrm{H}$ demonstrated an increased capacity of $\mathrm{Co}_{3} \mathrm{O}_{4}\left(25 \% \mathrm{Fe}_{3} \mathrm{O}_{4}\right)$ NPs to generate reactive superoxide free radicals compared to $\mathrm{Co}_{3} \mathrm{O}_{4}\left(0 \%\right.$ and $\left.75 \% \mathrm{Fe}_{3} \mathrm{O}_{4}\right) \mathrm{NPs}$. Increasing amounts of Fe-doping using $\mathrm{Fe}_{3} \mathrm{O}_{4}$ led to a significant decrease in scavenging capacity of $\mathrm{Co}_{3} \mathrm{O}_{4}$ NPs. $\mathrm{Co}_{3} \mathrm{O}_{4}\left(0 \%, 25 \%\right.$ and $\left.75 \% \mathrm{Fe}_{2} \mathrm{O}_{3}\right)$ NPs had a similar ROS generation and scavenging capacity (Table 1$)$. 


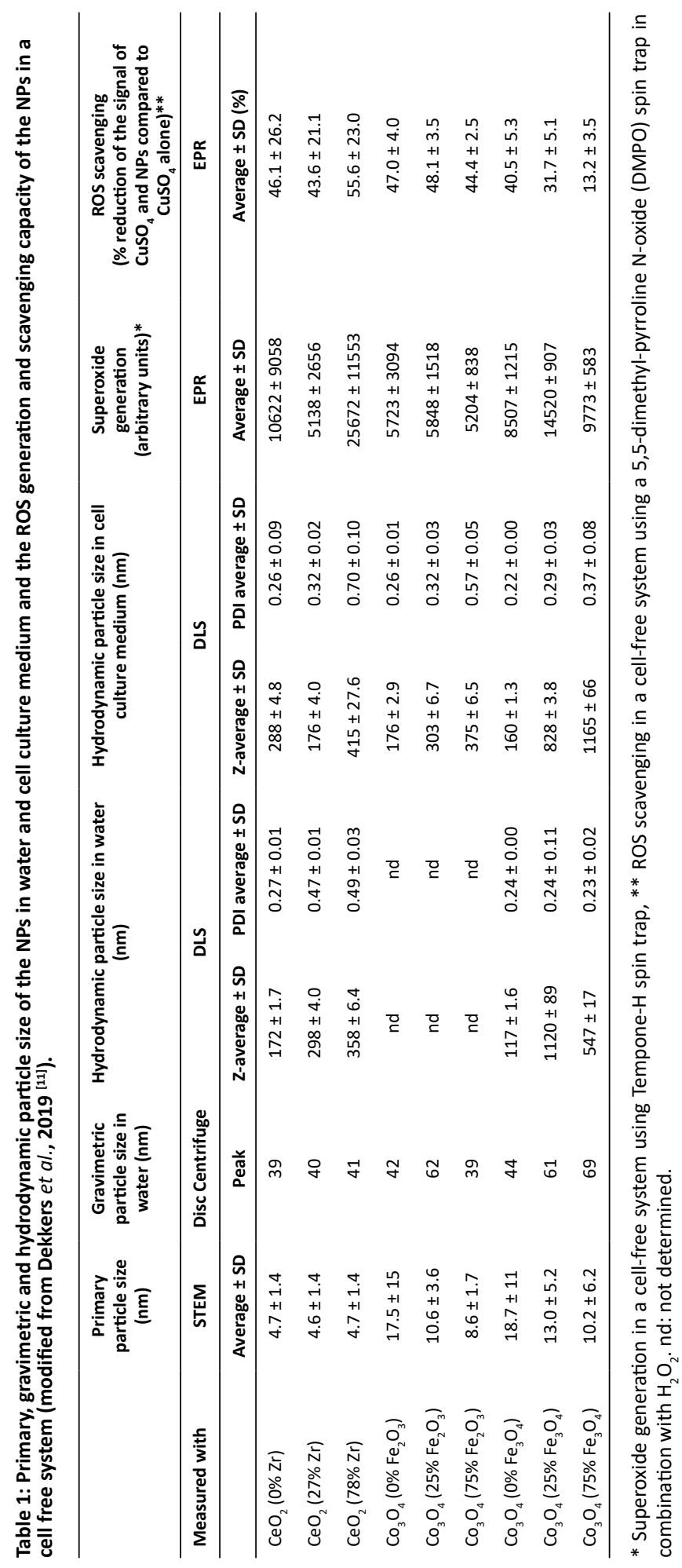


The Scanning Transmission Electron Microscope (STEM) images show differences in the morphology of undoped $\mathrm{CeO}_{2} \mathrm{NPs}$ in water (a) and cell culture medium (b) and between the two different batches of undoped $\mathrm{Co}_{3} \mathrm{O}_{4}$ NPs in water ( $c$ and d) (see Figure 1).
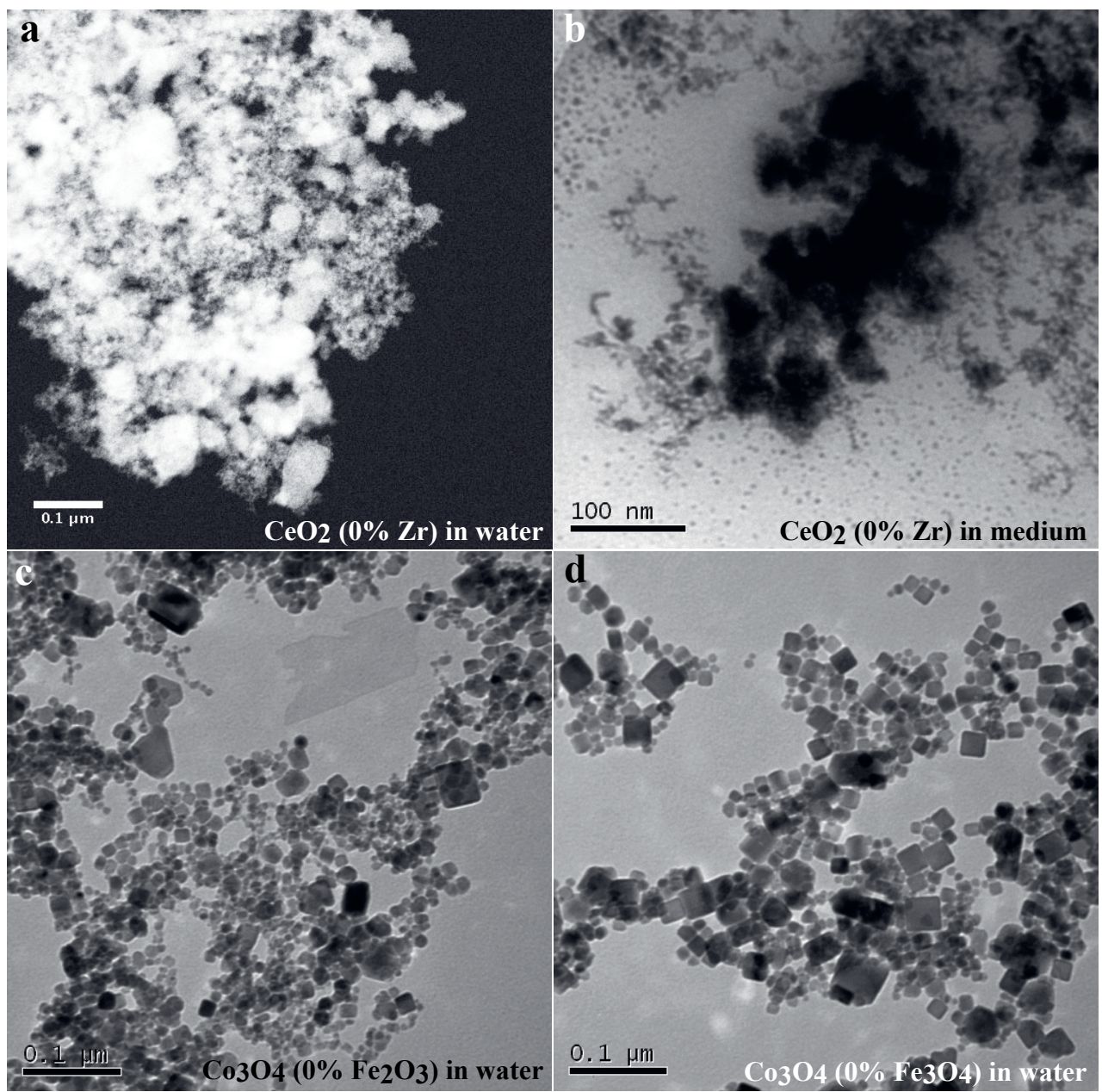

Figure 1: TEM images of undoped $\mathrm{CeO}_{2} \mathrm{NPs}$ in water (a) and undoped $\mathrm{CeO}_{2} \mathrm{NPs}$ in cell culture medium (b) and the two different batches of undoped $\mathrm{Co}_{3} \mathrm{O}_{4}$ NPs in water ( $c$ and d).

All NPs were tested for endotoxin contamination using either a Limulus Amoebocyte Lysate Assay (chromogenic kinetic LAL assay) or Ultra High Performance Liquid Chromatography coupled with Mass Spectrometry (UHPLC-MS/MS) determination ${ }^{[25]}$. Endotoxin levels were below the detection limit of 0.3 Endotoxin Units $/ \mathrm{mL}$ of the UHPLC-MS/MS method for most NPs, except for the $\mathrm{Co}_{3} \mathrm{O}_{4}\left(75 \% \mathrm{Fe}_{3} \mathrm{O}_{4}\right)$ and $\mathrm{Co}_{3} \mathrm{O}_{4}\left(25 \% \mathrm{Fe}_{3} \mathrm{O}_{4}\right)$ NPs for which the endotoxin levels were $0.4 \mathrm{EU} / \mathrm{mL}$ sample (of dispersions with a concentration of $128 \mu \mathrm{g} \mathrm{NPs} / \mathrm{mL}$ ). This was below the recommended endotoxin limits for preclinical research in animal models $(5$ 
$\mathrm{IU} / \mathrm{kg} \mathrm{bw} /$ day or $36 \mathrm{IU} / \mathrm{mL}$ for mice with a body weight of $30 \mathrm{~g}$ and a daily dose of $100 \mu \mathrm{L}$ ) and exposure limits for intravenous administration of medicinal products $(5 \mathrm{IU} / \mathrm{kg} \mathrm{bw} / \mathrm{h})^{[26]}$.

\subsection{In vitro deposition}

In vitro deposition on cells was determined using part 2 and 3 of the protocol described in DeLoid et al., $2017^{[27]}$. The first part of the protocol, the preparation of the NP dispersions was not needed, since all NPs were already produced in dispersion. To re-disperse any potential agglomerates, the NP dispersions were vortexed for 10 seconds and sonicated for 5 minutes in an ultrasonic bath (Branson CPX2800, $40 \mathrm{KHz}, 100 \mathrm{~W}$ ) before dilution in cell culture media to a concentration of $128 \mu \mathrm{g} / \mathrm{mL}$. The determination of the volume weighted size distribution and effective density of the NPs in cell culture medium was determined according to part 2 of the protocol, using DLS and the volumetric centrifugation method. According to part 3 of the protocol, the volume weighted size distribution in cell culture medium, the raw density and the effective density were used to estimate the percentage of the NPs deposited on the bottom of the cell culture well with the distorted grid (DG) model of Harvard University ${ }^{[28]}$. Other experimental conditions entered as input parameters in the DG model were: the use of a 96 wells plate, $200 \mu \mathrm{l}$ cell culture medium per well, a temperature of $37^{\circ} \mathrm{C}$, a viscosity of $0.00074 \mathrm{~Pa} \mathrm{~s}{ }^{[29]}$, a density of $1.008 \mathrm{~g} / \mathrm{cm}^{3}$ of the cell culture medium, a concentration $128 \mu \mathrm{g} \mathrm{NPs} / \mathrm{mL}$ cell culture medium and a duration of 48 $h$ for the experiment.

\subsection{Cell culture and treatment}

Wild type as well as NLRP3- or ASC-deficient THP-1 macrophages were cultured at $37^{\circ} \mathrm{C}$ and $5 \% \mathrm{CO}_{2}$ in RPMI 1640 (Gibco) with 25mM Hepes and $2 \mathrm{mM} \mathrm{L-Glutamine,} 100 \mathrm{U} / \mathrm{mL}$ Penicillin, $100 \mu \mathrm{g} / \mathrm{mL}$ Streptomycin (Pen/Strep; Gibco) and 10\% heat inactivated Foetal Calf Serum (FCS; Gibco), in a T75 flask in $15 \mathrm{~mL}$. The THP-1 cell line was a kind gift from Samantha Kloet at Wageningen University. The NLRP3- and ASC-deficient THP-1 macrophages were obtained from InvivoGen. The THP-1 macrophages were cultured twice a week during which the cell concentration was adjusted to approximately $2 \times 10^{5}$ cells $/ \mathrm{mL}$. Hygromycin B (Hygrogold, InvivoGen) was added to the cell culture medium of the NLRP3- or ASC-deficient THP-1 macrophages as a selective antibiotic at a concentration $200 \mu \mathrm{g} / \mathrm{mL}$.

The THP-1 macrophages $\left(5.0 \times 10^{5}\right.$ cells $\left./ \mathrm{mL}\right)$ were differentiated with $100 \mathrm{ng} / \mathrm{mL}$ phorbol 12-myristate 13-acetate (PMA; Sigma) in $200 \mu$ cell suspension in a 96 -wells cell culture plate (sterile, F-bottom, with lid, CellStar) (Greiner bio-one) in an incubator at $37^{\circ} \mathrm{C}$ with $5 \% \mathrm{CO}_{2}$ in air. After $3 \mathrm{~h}$, the medium of the cells was changed with $100 \mu \mathrm{l}$ fresh medium without PMA and the cells were incubated for 20-24 h before exposure to NPs. After this resting period, $100 \mu \mathrm{l}$ fresh medium with different NP concentrations was added to each of the wells to obtain a range of concentrations from 0 to $128 \mu \mathrm{g} / \mathrm{mL}$ of NPs. Following this, the 
cells were incubated for another $48 \mathrm{~h}$ before measuring the cell viability (with WST-1) and collecting the supernatant for measuring IL-1 $\beta$ levels.

Dendritic cells (DC) were obtained and cultured as previously described ${ }^{[3]}$. Briefly, they were derived from buffy coats obtained from Sanquin (Amsterdam, the Netherlands). Peripheral blood mononuclear cells were isolated from buffy coats by density centrifugation (Lymphoprep; Axis Shield, Oslo, Norway). The cells were washed, harvested, and resuspended in RPMI-1640 (Gibco, Grand Island, NY, USA) supplemented with 2\% heatinactivated human serum (Harlan, Boxmeer, the Netherlands), $100 \mu \mathrm{g} / \mathrm{mL}$ streptomycin, $100 \mathrm{IU} / \mathrm{mL}$ penicillin, and $0.3 \mathrm{mg} / \mathrm{mL}$ L-glutamine. They were seeded in tissue culture flasks (Corning, Amsterdam, the Netherlands) and were incubated for $1 \mathrm{~h}$. After this incubation non-attached cells were removed by rinsing with warm $\left(37^{\circ} \mathrm{C}\right) \mathrm{PBS}$ and medium was added (RPMI-1640 supplemented with 10\% heat-inactivated Foetal Calf Serum ("FCS"; Hyclone; GE Healthcare, Logan, UT, USA), streptomycin, penicillin, L-glutamine, $500 \mathrm{U} / \mathrm{mL}$ GM-CSF and $250 \mathrm{U} / \mathrm{mL}$ IL-4). At day 3, half the volume of the medium was replaced with fresh medium with fresh cytokines. At day 6 , the immature DC were harvested by rinsing with cold PBS and adding cold PBS to the flask, placing the flask in the refrigerator for one hour and tapping the flask to detach the cells. Hundred $\mu \mathrm{L}$ of the freshly prepared cell suspension $\left(6 \times 10^{5}\right.$ cells $/ \mathrm{mL}$ ) was then added to each well of a 96-wells plate and placed in an incubator at $37^{\circ} \mathrm{C}$ in a humidified atmosphere containing $5 \% \mathrm{CO}_{2}$ in air awaiting NP exposure. The DC were exposed by adding $100 \mu \mathrm{l}$ medium with different NP concentrations to each of the wells to obtain a range of concentrations from 0 to $128 \mu \mathrm{g} / \mathrm{mL}$ of NPs. Following this, the cells were incubated for $48 \mathrm{~h}$ before measuring the cell viability (using the CFDA assay) and collecting the supernatant for measuring IL-12p40 levels.

\subsection{Measuring cell viability}

The cell viability of wild type and NLRP3- or ASC-deficient THP-1 macrophages was measured using the WST-1 conversion assay. After 48 hours exposure to NPs, $20 \mu \mathrm{L}$ of WST-1 reagent (Roche Diagnostics $\mathrm{GmbH}$ ) was added to each well and incubated at $37^{\circ} \mathrm{C}$ for 1,2 and $3 \mathrm{~h}$ before measurement. The absorbance $(440 \mathrm{~nm})$ was measured with a Spectramax M2 spectrophotometer.

The cell viability of DCs was measured using the CFDA assay. After $48 \mathrm{~h} 5$-Carboxyfluorescein Diacetate, Acetoxymethyl Ester (5AM-CFDA) (Fisher Scientific) was added to each well (final concentration $4 \mu \mathrm{M}$ ). The DCs were incubated at $37^{\circ} \mathrm{C}$ and fluorescence (excitation $491 \mathrm{~nm}$, emission $530 \mathrm{~nm}$ ) was measured after 1, 2 and $3 \mathrm{~h}$ with a Spectramax M2 spectrophotometer.

Interference of the NPs with the read out systems of the WST-1 and 5AM-CFDA assays was evaluated by including additional wells with only NPs in cell culture medium (without cells) in these assays. Samples were measured in triplicate, and in duplicate for NP interference 
in wells without cells. To correct for any interference, the signal of the wells measuring the NP interference was subtracted from the signal of the wells with NPs and cells. If the interference was larger than $20 \%$ of the signal, the interference was considered too large for a reliable measurement and the data was not used for further analyses. After subtraction of the interference, the signal of the different dose levels was normalised to the signal of the control wells (without NP, but with cells).

\subsection{Detection of cytokines}

Supernatants of exposed cells were collected after centrifuging at $4^{\circ} \mathrm{C}$ for $5 \mathrm{~min}$ at a speed of $1200 \mathrm{RPM}$, and stored at $-80^{\circ} \mathrm{C}$ until analysis. THP-1 cell supernatant, harvested after $48 \mathrm{~h}$ exposure to NPs, was tested for human IL-1 $\beta$ using the Human IL-1 $\beta$ ( 2 nd Gen) Ready-SETGo! ELISA kit (eBioscience) according to the manufacturer's instructions. The samples were diluted 2 to 25 times before loading on the IL-1 $\beta$ ELISA plate.

DC supernatant, harvested after $48 \mathrm{~h}$ exposure to NPs, was tested for human IL-12p40 using the Human IL-12p40 ELISA set (BD OptEIA), according to the manufacturer's instructions. The samples were not diluted.

\subsection{Statistical analysis and dose response modelling}

The cell viability and IL-1 $\beta$ production data of at least three THP-1 experiments were combined, plotted and used for non-linear regression analyses (curve-fitting) for each NP in Graph Pad Prism (version 7.04). For the DC experiments, the cell viability and IL-12p40 production data of each experiment (each individual donor) was plotted and used for nonlinear regression analyses (curve-fitting) for each NP.

For further statistical analysis and dose response modelling with PROAST software ${ }^{\text {[30] }}$ (www.rivm.nl/proast) (version 63.5), the same data was used as for the analysis in Graph Pad Prism. Statistical testing was performed by fitting the best dose response curve and determining the critical effect dose (CED) and the $90 \%$ confidence interval. To determine the CED, a critical effect size (CES) of $20 \%$ for cell viability and $5 \%$ for cytokine levels (IL- $1 \beta$ and IL-12p40 concentrations) was used. PROAST uses the Hill and Exponential models to predict the "best fit" dose response curve by comparing the log likelihood value of each selected model with the full model. The log likelihood value relates to the goodness of fit for the dose response curve: the higher the value, the better the model. A conservative $90 \%$ confidence interval was determined as the range of the lowest $5 \%$ level and highest $95 \%$ level of the $90 \%$ confidence intervals of the best fitted Hill and the best fitted Exponential model. Differences between the CEDs of the different NPs are statistically significantly different if the $95 \%$ upper and lower confidence limits of the CEDs from the NPs do not overlap. 


\section{Results}

\subsection{In vitro deposition}

Zr-doping of the $\mathrm{CeO}_{2} \mathrm{NPs}$ decreased the volume weighted hydrodynamic size in cell culture medium, resulting in a slight decrease in the deposited dose of $\mathrm{CeO}_{2}(27 \% \mathrm{Zr}) \mathrm{NPs}$ after $48 \mathrm{~h}$. A more pronounced influence was observed for both types of Fe-doping. Both types of Fedoping doubled or tripled the volume weighted hydrodynamic size of the $\mathrm{Co}_{3} \mathrm{O}_{4}$ NPs in cell culture medium, resulting in a two- to three-fold increase in the estimated deposited dose after $48 \mathrm{~h}$ (see Table 2).

Table 2: Hydrodynamic particle size and estimated deposition after $48 \mathrm{~h}$ as calculated with the distorted grid model.

\begin{tabular}{|c|c|c|c|c|c|c|c|}
\hline & \multicolumn{3}{|c|}{$\begin{array}{l}\text { Hydrodynamic particle size in cell } \\
\text { culture medium }(\mathrm{nm}) \text { by DLS }\end{array}$} & \multirow{2}{*}{$\begin{array}{c}\text { Raw } \\
\text { density } \\
\left(\mathrm{g} / \mathrm{cm}^{3}\right)\end{array}$} & \multirow{2}{*}{$\begin{array}{c}\text { Effective } \\
\text { density } \\
\left(\mathrm{g} / \mathrm{cm}^{3}\right)\end{array}$} & \multicolumn{2}{|c|}{$\begin{array}{l}\text { Deposited dose } \\
\text { after } 48 \mathrm{~h}^{*}\end{array}$} \\
\hline & $\begin{array}{l}\text { Z-average } \pm \\
\text { SD }(\mathrm{nm})\end{array}$ & $\mathrm{PDI} \pm \mathrm{SD}$ & $\begin{array}{l}\text { Volume-weighted } \\
\text { average } \pm S D(\mathrm{~nm})\end{array}$ & & & (\%) & $\left(\mu \mathrm{g} / \mathrm{cm}^{2}\right)$ \\
\hline $\mathrm{CeO}_{2}(0 \% \mathrm{Zr})$ & $288 \pm 4.8$ & $0.26 \pm 0.09$ & $731 \pm 185$ & 7.215 & 1.601 & 69 & 54 \\
\hline $\mathrm{CeO}_{2}(27 \% \mathrm{Zr})$ & $176 \pm 4.0$ & $0.31 \pm 0.02$ & $484 \pm 82$ & 6.801 & 1.754 & 51 & 39 \\
\hline $\mathrm{CeO}_{2}(78 \% \mathrm{Zr})$ & $415 \pm 27.6$ & $0.70 \pm 0.10$ & $558 \pm 47$ & 6.018 & 2.129 & 68 & 53 \\
\hline $\mathrm{Co}_{3} \mathrm{O}_{4}\left(0 \% \mathrm{Fe}_{2} \mathrm{O}_{3}\right)$ & $176 \pm 2.9$ & $0.26 \pm 0.01$ & $522 \pm 114$ & 6.110 & 1.634 & 51 & 39 \\
\hline $\mathrm{Co}_{3} \mathrm{O}_{4}\left(25 \% \mathrm{Fe}_{2} \mathrm{O}_{3}\right)$ & $303 \pm 6.7$ & $0.32 \pm 0.03$ & $1020 \pm 267$ & 5.893 & 1.739 & 86 & 67 \\
\hline $\mathrm{Co}_{3} \mathrm{O}_{4}\left(75 \% \mathrm{Fe}_{2} \mathrm{O}_{3}\right)$ & $375 \pm 6.5$ & $0.57 \pm 0.05$ & $1316 \pm 106$ & 5.458 & 1.327 & 90 & 70 \\
\hline $\mathrm{Co}_{3} \mathrm{O}_{4}\left(0 \% \mathrm{Fe}_{3} \mathrm{O}_{4}\right)$ & $160 \pm 1.3$ & $0.22 \pm 0.00$ & $381 \pm 44$ & 6.110 & 1.564 & 29 & 22 \\
\hline $\mathrm{Co}_{3} \mathrm{O}_{4}\left(25 \% \mathrm{Fe}_{3} \mathrm{O}_{4}\right)$ & $828 \pm 3.8$ & $0.29 \pm 0.03$ & $1372 \pm 45$ & 5.833 & 1.353 & 94 & 73 \\
\hline $\mathrm{Co}_{3} \mathrm{O}_{4}\left(75 \% \mathrm{Fe}_{3} \mathrm{O}_{4}\right)$ & $1165 \pm 66$ & $0.37 \pm 0.08$ & $1465 \pm 57$ & 5.278 & 1.626 & 99 & 76 \\
\hline
\end{tabular}

* Deposited dose is estimated for the situation in which $200 \mu$ of the highest concentration $(128 \mu \mathrm{g} / \mathrm{mL}$ or $80 \mu \mathrm{g} /$ $\mathrm{cm}^{2}$ ) per well is administered.

\subsection{In vitro cell viability and cytokine production by THP-1 macrophages}

After exposure of THP-1 macrophages to the various $\mathrm{CeO}_{2} \mathrm{NPs}$ (Zr-doped and undoped) a dose-related increase in IL-1 $\beta$ production was already observed at non-cytotoxic dose levels. No increase in IL-1 $\beta$ induction was observed and no dose-response curve could be fitted to the data of any of the $\mathrm{Co}_{3} \mathrm{O}_{4} \mathrm{NPs}$ (Fe-doped or undoped) (Figure 2). Only very limited to no IL-1 $\beta$ production was observed in the NLRP3- or ASC-deficient THP-1 macrophages after exposure to any of the NPs. In addition, no or only very low IL-1 $\beta$ production was seen in NLRP3- and ASC-deficient THP-1 macrophages without NP exposure or with exposure to the positive control $\mathrm{SiO}_{2} \mathrm{NPs}$ (Figure 3). 
a
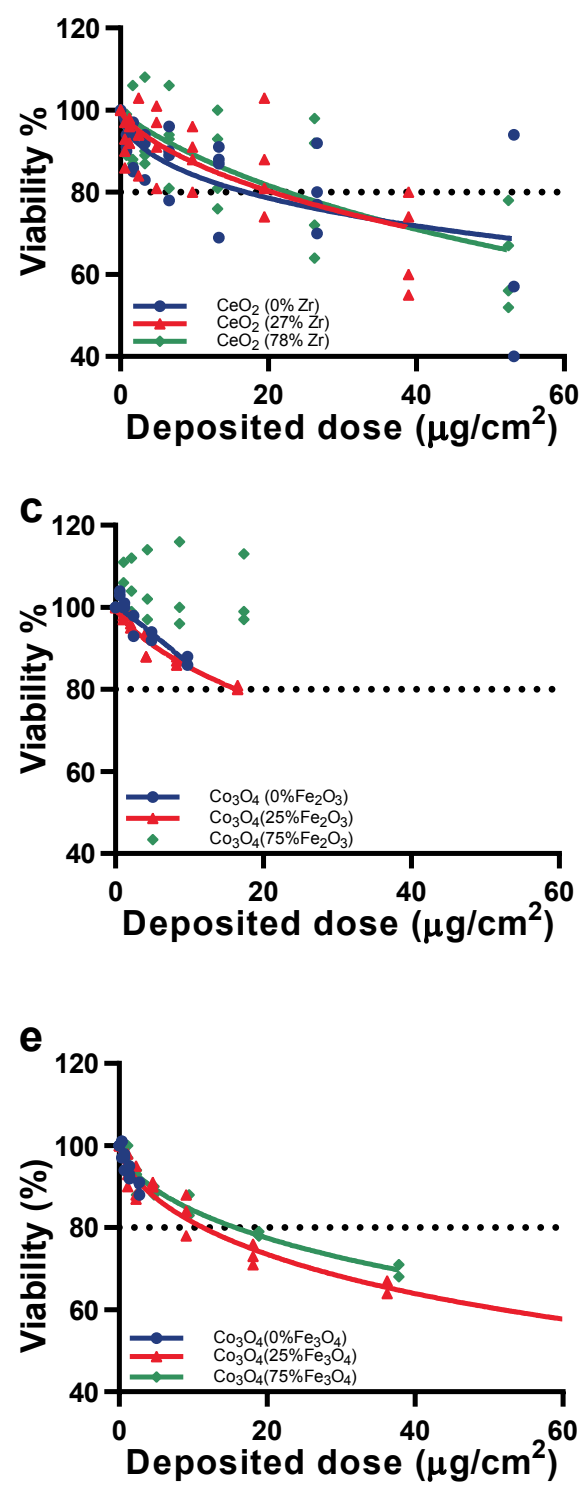

b
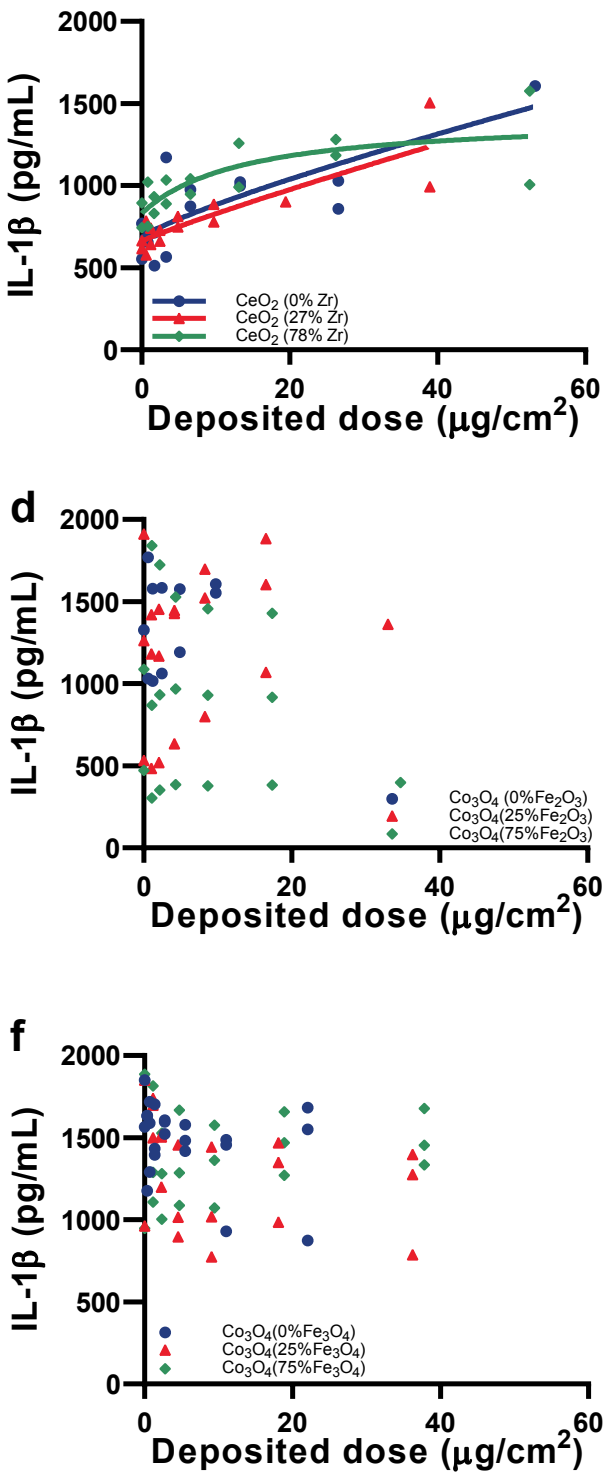

Figure 2: Cell viability and IL-1 $\beta$ production in THP-1 macrophages exposed to $\mathrm{Zr}$-doped $\mathrm{CeO}_{2} \mathrm{NPs}(\mathrm{a}$ and $\mathrm{b}$ ), Fedoped $\mathrm{Co}_{3} \mathrm{O}_{4}$ NPs (using $\mathrm{Fe}_{2} \mathrm{O}_{3}$ ) (c and d) and Fe-doped $\mathrm{Co}_{3} \mathrm{O}_{4} \mathrm{NPs}$ (using $\mathrm{Fe}_{3} \mathrm{O}_{4}$ ) (e and f) for $48 \mathrm{~h}(\mathrm{n}=3$ ). The doseresponse curves show a clear increase in IL-1 $\beta$ production for all $\mathrm{CeO}_{2} \mathrm{NPs}$ already at non-cytotoxic dose levels. No increase in IL-1 $\beta$ production was observed after exposure to any of the Fe-doped $\mathrm{Co}_{3} \mathrm{O}_{4} \mathrm{NPs}$ and no dose-response curves could be fitted to these data. 
NLRP3-def THP-1 cells

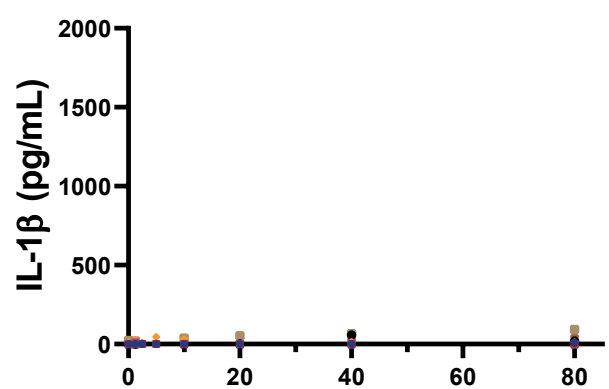

Administered dose $\left(\mu \mathrm{g} / \mathrm{cm}^{2}\right)$
ASC-def THP-1 cells

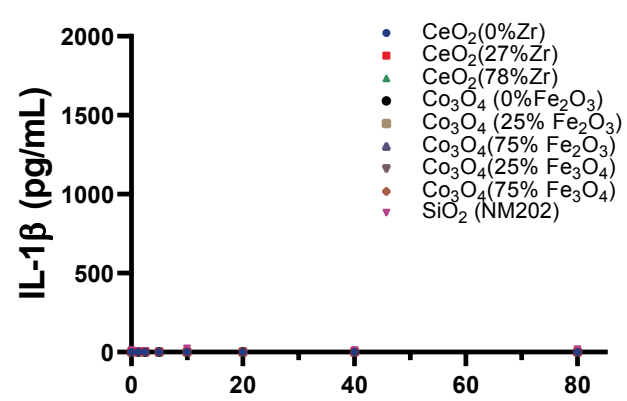

Administered dose $\left(\mu \mathrm{g} / \mathrm{cm}^{2}\right)$

Figure 3: IL-1 $\beta$ production in NLRP3- and ASC-deficient THP-1 macrophages exposed to Zr-doped CeO ${ }_{2}$ NPs, Fe-doped $\mathrm{Co}_{3} \mathrm{O}_{4} \mathrm{NPs}$ (using $\mathrm{Fe}_{2} \mathrm{O}_{3}$ or $\mathrm{Fe}_{3} \mathrm{O}_{4}$ ) and $\mathrm{SiO}_{2} \mathrm{NPs}(\mathrm{NM} 202)$ for $48 \mathrm{~h}(\mathrm{n}=1)$. No or only very low IL-1 $\beta$ concentrations were measured.

Dose response modelling with PROAST confirmed that IL-1 $\beta$ production was already observed at non-cytotoxic dose levels, as the $\mathrm{CED}_{5 \%}$ of IL-1 $\beta$ production after exposure to $\mathrm{CeO}_{2} \mathrm{NPs}\left(\approx 0.01-3.2 \mu \mathrm{g} / \mathrm{cm}^{2}\right)$ was lower than the $\mathrm{CED}_{20 \%}$ of cell viability $\left(\approx 14-28 \mu \mathrm{g} / \mathrm{cm}^{2}\right)$. PROAST analysis could not reliably fit a dose response curve to the IL-1 $\beta$ production data of THP-1 macrophages exposed to $\mathrm{Co}_{3} \mathrm{O}_{4} \mathrm{NPs}$, confirming that there was no increase in IL-1 $\beta$ production for $\mathrm{Co}_{3} \mathrm{O}_{4}\left(0 \%, 25 \%\right.$ and $\left.75 \% \mathrm{Fe}_{2} \mathrm{O}_{3}\right)$ or $\mathrm{Co}_{3} \mathrm{O}_{4}\left(0 \%, 25 \%\right.$ and $\left.75 \% \mathrm{Fe}_{3} \mathrm{O}_{4}\right)$.

\subsection{In vitro cell viability and cytokine production by DCs}

A clear increase in IL-12p40 production was observed after exposure of DCs from all blood donors to $\mathrm{Co}_{3} \mathrm{O}_{4}\left(0 \% \mathrm{Fe}_{2} \mathrm{O}_{3}\right)(\mathrm{n}=3)$ and $\mathrm{Co}_{3} \mathrm{O}_{4}\left(25 \% \mathrm{Fe}_{2} \mathrm{O}_{3}\right)(\mathrm{n}=4)$ already at non-cytotoxic dose levels. DCs from all donors showed a clear to moderate increase in IL-12p40 production after exposure to $\mathrm{Co}_{3} \mathrm{O}_{4}\left(0 \% \mathrm{Fe}_{3} \mathrm{O}_{4}\right)(n=4)$ and $\mathrm{Co}_{3} \mathrm{O}_{4}\left(75 \% \mathrm{Fe}_{3} \mathrm{O}_{4}\right)(n=4)$. There was no increase in IL-12p40 production after exposure of DCs of any of the donors to undoped and Zr-doped $\mathrm{CeO}_{2} \mathrm{NPs}(\mathrm{n}=3), \mathrm{Co}_{3} \mathrm{O}_{4}\left(75 \% \mathrm{Fe}_{2} \mathrm{O}_{3}\right)(n=4)$ and $\mathrm{Co}_{3} \mathrm{O}_{4}\left(25 \% \mathrm{Fe}_{3} \mathrm{O}_{4}\right)(n=3)$. A dose response curve for both cell viability and IL-12p40 production after exposure of DCs from one of the donors to each of the NPs is shown in Figure 4. 
a

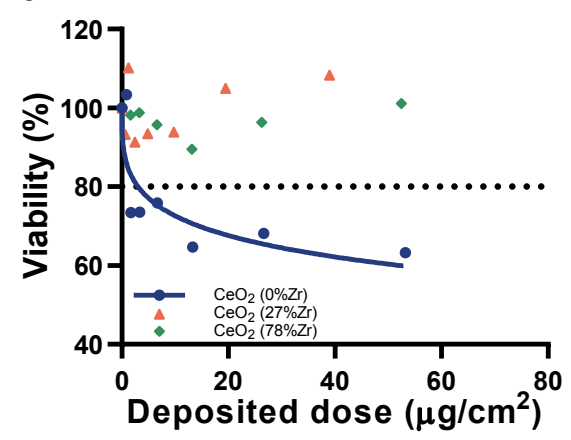

C

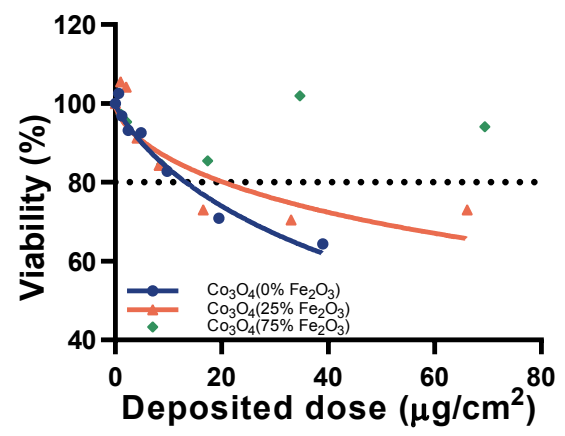

e

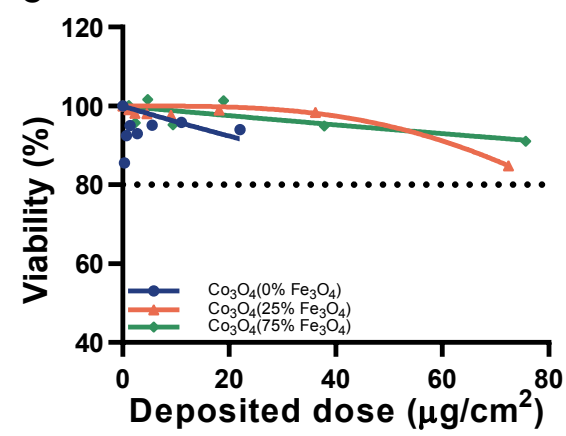

b

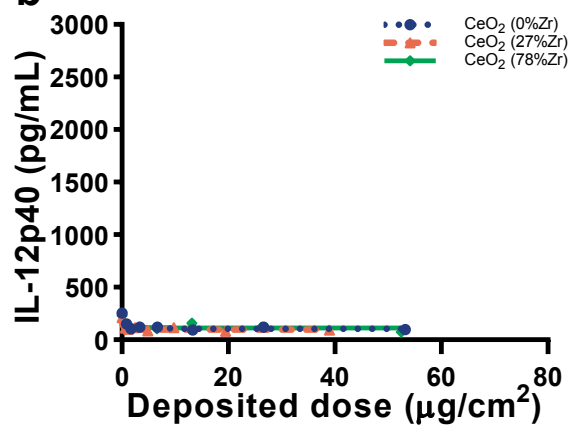

d

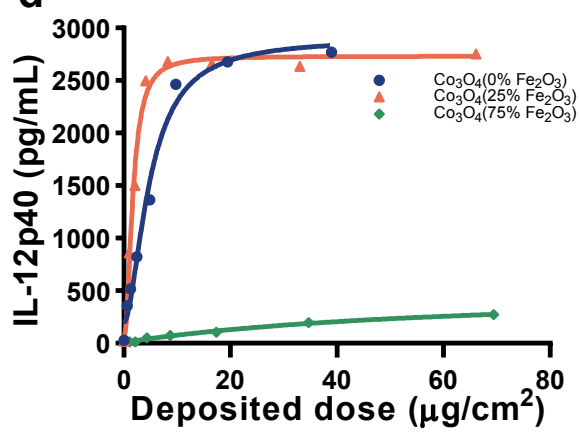

Figure 4: Representative dose-response curves of cell viability and IL-12p40 production in DC from one of the donors exposed to a) $\mathrm{Zr}$-doped $\mathrm{CeO}_{2} \mathrm{NPs}$, b) Fe-doped $\mathrm{Co}_{3} \mathrm{O}_{4} \mathrm{NPs}$ (using $\mathrm{Fe}_{2} \mathrm{O}_{3}$ ) and c) Fe-doped $\mathrm{Co}_{3} \mathrm{O}_{4} \mathrm{NPs}$ (using $\left.\mathrm{Fe}_{3} \mathrm{O}_{4}\right)$ for $48 \mathrm{~h}(\mathrm{n}=1)$. There was no increase in IL-12p40 production after exposure to undoped and $\mathrm{Zr}$-doped $\mathrm{CeO}_{2}$ $\mathrm{NPs}, \mathrm{Co}_{3} \mathrm{O}_{4}\left(75 \% \mathrm{Fe}_{2} \mathrm{O}_{3}\right)$ and $\mathrm{Co}_{3} \mathrm{O}_{4}\left(25 \% \mathrm{Co}_{3} \mathrm{O}_{4}\right)$, a clear increase in IL-12p40 production for $\mathrm{Co}_{3} \mathrm{O}_{4}\left(0 \% \mathrm{Fe}_{2} \mathrm{O}_{3}\right)$ and $\mathrm{Co}_{3} \mathrm{O}_{4}\left(25 \% \mathrm{Fe}_{2} \mathrm{O}_{3}\right)$ and a moderate increase in IL-12p40 production for $\left.\mathrm{Co}_{3} \mathrm{O}_{4}\left(0 \% \mathrm{Fe}_{3} \mathrm{O}_{4}\right)\right)$ and $\left.\mathrm{Co}_{3} \mathrm{O}_{4}\left(75 \% \mathrm{Fe}_{3} \mathrm{O}_{4}\right)\right)$ at non-cytotoxic dose levels. 
PROAST analysis confirmed that the increases in IL-12p40 production were already observed at non-cytotoxic dose levels. For $\mathrm{Co}_{3} \mathrm{O}_{4}\left(0 \% \mathrm{Fe}_{2} \mathrm{O}_{3}\right)$ and $\mathrm{Co}_{3} \mathrm{O}_{4}\left(25 \% \mathrm{Fe}_{2} \mathrm{O}_{3}\right)$ the $\mathrm{CED}_{5 \%}$ of IL$12 \mathrm{p} 40$ production was lower $\left(\approx 0.001-0.03 \mu \mathrm{g} / \mathrm{cm}^{2}\right)$ than the $\mathrm{CED}_{20 \%}$ of cell viability $(\approx 5-15$ $\left.\mu \mathrm{g} / \mathrm{cm}^{2}\right)$. For $\mathrm{Co}_{3} \mathrm{O}_{4}\left(0 \% \mathrm{Fe}_{3} \mathrm{O}_{4}\right)$ and $\mathrm{Co}_{3} \mathrm{O}_{4}\left(75 \% \mathrm{Fe}_{3} \mathrm{O}_{4}\right)$ the $\mathrm{CED}_{5 \%}$ of IL-12p40 production was lower $\left(\approx 0-0.5 \mu \mathrm{g} / \mathrm{cm}^{2}\right)$ than the $\mathrm{CED}_{20 \%}$ of cell viability $\left(\approx 16-\infty \mu \mathrm{g} / \mathrm{cm}^{2}\right)$. No increase in IL$12 \mathrm{p} 40$ production was observed for $\mathrm{Co}_{3} \mathrm{O}_{4}\left(75 \% \mathrm{Fe}_{2} \mathrm{O}_{3}\right), \mathrm{Co}_{3} \mathrm{O}_{4}\left(25 \% \mathrm{Fe}_{3} \mathrm{O}_{4}\right)$ and the undoped and $\mathrm{Zr}$-doped $\mathrm{CeO}_{2}$ NPs.

\section{Discussion}

\subsection{NP characterization}

No consistent increase or decrease in primary or gravimetric particle size was observed due to the $\mathrm{Zr}$-doping of the $\mathrm{CeO}_{2}$ or Fe-doping of the $\mathrm{Co}_{3} \mathrm{O}_{4}$ NPs. However, with a few exceptions, doping of the $\mathrm{CeO}_{2}$ and $\mathrm{CO}_{3} \mathrm{O}_{4}$ NPs did increase the hydrodynamic particle size in water and in cell culture medium (Table 1). Both the DLS and STEM analyses indicate that the agglomerated particle size of the same NPs is generally larger in cell culture medium compared to water (Table 1 and Figure 1). However, the polydispersity index of some of the DLS measurements in cell culture medium was larger than 0.5 , indicating a large variability in the diameter of the particles within the dispersion (Table 2). In addition, the two different batches of the non-doped $\mathrm{Co}_{3} \mathrm{O}_{4}$ NPs showed differences in the primary and hydrodynamic particle size and shape (Table 1, Figure 1 and ${ }^{[24]}$ ). These observed variations and differences in the primary, gravimetric and hydrodynamic particle size and shape of the NPs may affect their ability to generate ROS, their deposition in the in vitro assays and subsequent results in the in vitro assays (cytotoxicity and cytokine induction).

Based on our data (Table 1), the influence of doping or particle size on the ability of the NPs to either generate or scavenge ROS is limited. Most NPs showed neither a decrease nor an increase in their ability to either generate or scavenge ROS with increasing amounts of doping or with increasing hydrodynamic particle diameter in water. Only Fe-doping using $\mathrm{Fe}_{3} \mathrm{O}_{4}$ led to a significant decrease in the scavenging capacity of $\mathrm{Co}_{3} \mathrm{O}_{4}$ NPs with increasing amounts of doping and increasing hydrodynamic size in cell culture medium. This is in accordance with the ROS generating capacity of $\mathrm{Fe}_{3} \mathrm{O}_{4}$ NPs in bacterial cells observed by Al-Shabib et al. ${ }^{[31]}$. In our previous paper studying the same NPs in vivo, we proposed that differences in particle size and aggregation may counterbalance any doping-related differences in free radical generation or scavenging ${ }^{[11]}$. However, to demonstrate this, more data on the ability to generate or scavenge ROS of the same NPs with varying particle and aggregated sizes are needed. 


\subsection{In vitro deposition}

The amount of doping influenced the estimated deposition of the NPs in the in vitro assays for the Fe-doped $\mathrm{CO}_{3} \mathrm{O}_{4}$, but not the $\mathrm{Zr}$-doped $\mathrm{CeO}_{2}$ NPs (Table 2). Since the estimated deposition in the in vitro assays is mainly determined by the hydrodynamic particle size in cell culture medium, an increase in the hydrodynamic particle size resulted in an increase in the estimated deposition in the in vitro assays for all NPs, especially when expressed as volume weighted averages (Table 2). To take the differences in deposition into account, the dose response curves were plotted using the deposited (i.e. biological effective) dose instead of the administered dose. The use of the deposited dose led to slightly different dose-response curves compared to the use of the administered concentration of the NPs in the cell culture medium (data not shown). In our study we preferred the use of the deposited dose, since the dose that reaches the cells and not the concentration of NPs in the cell culture medium drives the biological effect ${ }^{[28]}$.

\subsection{Inflammasome activation}

The dose response curves of IL-1 $\beta$ production of THP-1 macrophages exposed to $0 \%, 27 \%$ and $78 \% \mathrm{Zr}$ doped $\mathrm{CeO}_{2} \mathrm{NPs}$, indicated that inflammasome activation occurred at lower dose levels after exposure to $\mathrm{CeO}_{2}(78 \% \mathrm{Zr})$ as compared to $\mathrm{CeO}_{2}(0 \% \mathrm{Zr})$ or $\mathrm{CeO}_{2}(27 \%$ $\mathrm{Zr}$ ). A possible explanation for this difference might be the different capacity of the NPs to generate ROS, which is larger for $\mathrm{CeO}_{2}\left(78 \% \mathrm{Zr}\right.$ ) compared to $\mathrm{CeO}_{2}(0 \% \mathrm{Zr})$ or $\mathrm{CeO}_{2}(27 \% \mathrm{Zr})$ (Table 1). This concurs with the study by Zhou et al. which showed that ROS is an important mediator of inflammasome activation ${ }^{[32]}$. This is however not supported by their difference in scavenging capacity which also seemed higher for $\mathrm{CeO}_{2}\left(78 \% \mathrm{Zr}\right.$ ) compared to $\mathrm{CeO}_{2}(0 \%$ $\mathrm{Zr}$ ) or $\mathrm{CeO}_{2}(27 \% \mathrm{Zr}$ ) (Table 1). This may suggest that ROS generation is more important for inflammasome activation than scavenging capacity. No increase in IL-1 $\beta$ production was observed after exposure to any doped or undoped $\mathrm{Co}_{3} \mathrm{O}_{4} \mathrm{NPs}$, indicating that exposure of THP-1 macrophages to any of the $\mathrm{Co}_{3} \mathrm{O}_{4}$ NPs does not result in inflammasome activation. No increase in IL-1 $\beta$ production was observed in the ASC- and NLRP3-deficient cell lines after exposure to $\mathrm{CeO}_{2}(0 \%, 27 \%$ and $78 \% \mathrm{Zr}$ ) (Figure 3) indicating that the NLRP3 inflammasome is responsible for a large part of the IL-1 $\beta$ production. The relatively high IL-1 $\beta$ production observed in non-exposed wild type THP-1 macrophages in the absence of NPs exposure was unexpected, however comparable IL-1 $\beta$ background levels were observed in a study by a different group, also using THP-1 macrophages ${ }^{[33]}$. Differences in background levels of IL-1 $\beta$ between different studies can be due to differences in cell stimulation, cell numbers, incubation times, passage number of the cells, etc. The observed variability in background levels of IL-1 $\beta$ within this study are probably due to differences in passage number of the cells. No major differences in the cell viability of THP-1 macrophages exposed to doped and undoped $\mathrm{CeO}_{2}$ and $\mathrm{Co}_{3} \mathrm{O}_{4}$ NPs were observed (Figure 2). 
In contrast to our in vitro experiments in THP-1 macrophages, most other in vitro studies showed no inflammasome activation after exposure of macrophages to $\mathrm{CeO}_{2} \mathrm{NPs}^{[9,34-36]}$. Only one other in vitro study with THP-1 macrophages found increased IL-1 $\beta$ production after $\mathrm{CeO}_{2} \mathrm{NP}$ exposure ${ }^{[37]}$. Several other studies have demonstrated $\mathrm{CeO}_{2} \mathrm{NP}$ to be the only rare earth oxide which does not induce IL-1 $\beta$ in vitro ${ }^{[9,10]}$. The authors of these studies suggest that this might be due to the reduced dissolution of $\mathrm{CeO}_{2} \mathrm{NPs}$ under acidifying conditions such as in lysosomes, which prevents the release of rare earth ions that bind to phosphates leading to the formation of urchin-shaped structures, causing lysosomal membrane damage and subsequent NLRP3 inflammasome activation ${ }^{[9,10]}$. Although no urchin-shaped structures were observed after exposure to $\mathrm{CeO}_{2} \mathrm{NP}$ in vitro, in vivo intravenous $\mathrm{CeO}_{2} \mathrm{NP}$ exposure has been shown to produce Ce-phosphate nanoneedles in the spleen, while intratracheal $\mathrm{CeCl}_{3}$ exposure led to the formation of Ce-phosphate rod-shaped nanoneedles and spider-like structures in the lung ${ }^{[38,39]}$. Differences in the experimental conditions between the different in vitro assays may have led to different dissolution rates of the $\mathrm{CeO}_{2} \mathrm{NPs}$ and subsequent $\mathrm{IL}-1 \beta$ production.

In vivo experiments investigating inflammasome activation after $\mathrm{CeO}_{2} \mathrm{NPs}$ exposure also show contradicting results. Sager et al. ${ }^{[40]}$ found increased IL-1 $\beta$ levels in BALF of mice after pharyngeal aspiration of $\mathrm{CeO}_{2} \mathrm{NPs}$, while Lin et al. ${ }^{[9]}$ did not. These differences might be explained by differences in particle characteristics, dissolution rate, dispersion state and dispersion medium. Sager et al. ${ }^{[40]}$ showed that the pre-exposure dispersion status of $\mathrm{CeO}_{2}$ NPs is a factor that contributes to the post-exposure pulmonary response (cytotoxicity and IL-1 $\beta$ induction in BALF) after pharyngeal aspiration in mice. Another possible explanation is a difference in the (anti-)oxidant properties of the different $\mathrm{CeO}_{2} \mathrm{NPs} \mathrm{CeO}_{2} \mathrm{NPs}$ can act as both a scavenger and an inducer of ROS, depending on its immediate environment. Generation of intracellular ROS is one of the most studied pathways by which NPs can induce NLRP3 inflammasome activation ${ }^{[3,6,10]}$. Intracellular ROS may trigger inflammasome activation via lysosomal damage, cytochrome $C$ production or thioredoxin oxidation ${ }^{[6]}$. Since scavenging or oxidative properties of $\mathrm{CeO}_{2} \mathrm{NPs}$ are dependent on the $\mathrm{pH}$ of the immediate environment, differences in the experimental set up (e.g. use of different exposure medium, $\mathrm{pH}$, etc.) could explain the differences in the ability of $\mathrm{CeO}_{2}$ to induce inflammasome activation. However, also in this respect results from different studies are not consistent. Asati et al. ${ }^{[41]}$ found that while an acidic environment promoted the oxidative properties, a physiological environment promoted the anti-oxidant properties, whereas Sager et al. ${ }^{[00]}$ found the opposite relationship. In the ESR studies by Sager et al., $\mathrm{CeO}_{2} \mathrm{NPs}$ were shown to scavenge radicals in an acidic environment $\left(\mathrm{pH}^{\sim} 4.5\right)$ but not in a physiological environment ( $\mathrm{pH}$ 7.4). This was in line with their findings that $\mathrm{CeO}_{2} \mathrm{NPs}$ activated the NLRP3 inflammasome via lysosomal damage and cathepsin $\mathrm{B}$ release to a similar extent as NiO NPs did, but induced less IL-1 3 and IL-18 compared to $\mathrm{NiO} N P s$. In contrast to $\mathrm{CeO}_{2} \mathrm{NPs}, \mathrm{NiO}$ NPs showed scavenging properties in a physiological, but not in an acidic environment ${ }^{[40]}$. 
Our results are in line with those of Cho et al. ${ }^{[37]}$ and Sager et al. ${ }^{[40]}$ and suggest that the $\mathrm{CeO}_{2}$ NPs that induced most ROS species in water, also induced a higher amount of IL- $1 \beta$ via inflammasome activation in THP-1 macrophages

For $\mathrm{Co}_{3} \mathrm{O}_{4} \mathrm{NPs}$, the results of our in vitro experiments in THP-1 macrophages are in agreement with other in vitro studies that also showed no inflammasome activation in vitro ${ }^{[9,42]}$. To our knowledge, no in vivo studies investigating inflammasome activation with $\mathrm{Co}_{3} \mathrm{O}_{4} \mathrm{NPs}$ have been reported.

\subsection{DC maturation}

The dose response curves of cell viability of DC indicated that undoped $\mathrm{CeO}_{2} \mathrm{NPs}$ were more toxic compared to $\mathrm{Zr}$-doped $\mathrm{CeO}_{2} \mathrm{NPs}$. Undoped $\mathrm{Co}_{3} \mathrm{O}_{4} \mathrm{NPs}$ were more toxic compared to Fe-doped $\mathrm{Co}_{3} \mathrm{O}_{4}$, irrespective of the type of doping $\mathrm{Fe}_{2} \mathrm{O}_{3}$ or $\mathrm{Fe}_{3} \mathrm{O}_{4}$ ), or the percentage of doping. The dose response curves of IL-12p40 production indicated that DC maturation occurred after exposure to $\mathrm{Co}_{3} \mathrm{O}_{4}\left(0 \% \mathrm{Fe}_{2} \mathrm{O}_{3}\right), \mathrm{Co}_{3} \mathrm{O}_{4}\left(25 \% \mathrm{Fe}_{2} \mathrm{O}_{3}\right), \mathrm{Co}_{3} \mathrm{O}_{4}\left(0 \% \mathrm{Fe}_{3} \mathrm{O}_{4}\right)$ and $\mathrm{Co}_{3} \mathrm{O}_{4}$ $\left(75 \% \mathrm{Fe}_{3} \mathrm{O}_{4}\right)$, but not after exposure to $\mathrm{Co}_{3} \mathrm{O}_{4}\left(75 \% \mathrm{Fe}_{2} \mathrm{O}_{3}\right), \mathrm{Co}_{3} \mathrm{O}_{4}\left(25 \% \mathrm{Fe}_{3} \mathrm{O}_{4}\right)$ or any of the doped or undoped $\mathrm{CeO}_{2}$ NPs.

An increased capacity to generate ROS or a decreased capacity to scavenge ROS is expected to enhance the ability of the NPs to induce cytotoxicity and to generate IL-12p40 in DCs. However, the observed differences in the dose response curves cannot be explained by the different capacity of the NPs to generate or scavenge ROS in water, except maybe for the dose response curves of cell viability after exposure to $\mathrm{Co}_{3} \mathrm{O}_{4}\left(0 \%, 25 \%\right.$, and $\left.75 \% \mathrm{Fe}_{3} \mathrm{O}_{4}\right)$, which show a decrease in their capacity to scavenge ROS with increasing amounts of doping. A possible explanation why the results of this in vitro assay cannot be explained by the ability of the NPs to generate or scavenge ROS, might be that this ability to generate or scavenge ROS was measured in water and may be different under the conditions of the in vitro assay (in cell culture medium and in cells).

For $\mathrm{CeO}_{2} \mathrm{NPs}$, the results of our in vitro experiments on $\mathrm{DC}$ maturation are in agreement with other in vitro studies that also showed an absence of $\mathrm{DC}$ maturation in vitro ${ }^{[43,44]}$. For $\mathrm{Co}_{3} \mathrm{O}_{4^{\prime}}$ one other in vitro study investigated DC maturation and in that study no DC maturation was observed ${ }^{[43]}$. To our knowledge, no in vivo studies investigating $\mathrm{DC}$ maturation by $\mathrm{CeO}_{2}$ or $\mathrm{Co}_{3} \mathrm{O}_{4}$ NPs were reported.

\subsection{Comparison of in vitro and in vivo results}

To relate the dose levels from our in vitro studies to the dose levels from our in vivo study, we estimated the deposited dose in the pulmonary region of the lung in our in vivo study to be approximately $0.44 \mathrm{\mu g} / \mathrm{cm}^{2}$. For this estimate, we assumed that the entire intranasally administered dose (of $200 \mathrm{\mu g} / \mathrm{animal}$ ) deposited in the pulmonary region of the lung, which 
was assumed approximately $450 \mathrm{~cm}^{2}$ for mice weighing approximately $20 \mathrm{~g}$. This dose is far below the estimated deposited doses of the highest dose levels in our in vitro studies (between 22 and $76 \mu \mathrm{g} / \mathrm{cm}^{2}$, Table 2), but in the same range of the lowest dose levels (between 0.34 and $1.18 \mu \mathrm{g} / \mathrm{cm}^{2}$ ).

From both the in vitro and in vivo activities of the tested NPs (Table 3), it is apparent that the influence of doping on the immune responses is very limited, whereas the main chemical components $\left(\mathrm{CeO}_{2}\right.$ with $\mathrm{Zr}$ or $\mathrm{Co}_{3} \mathrm{O}_{4}$ with $\left.\mathrm{Fe}\right)$ does influence the type of immune responses in vitro and in vivo. To determine whether the observed immune responses in vivo can be predicted using the results of the in vitro assays is, however, not straightforward for both $\mathrm{CeO}_{2}$ or $\mathrm{Co}_{3} \mathrm{O}_{4} \mathrm{NPs}$ based on the data from the current in vitro study.

Table 3: Comparison of in vitro and in vivo data.

\begin{tabular}{|c|c|c|c|c|c|c|c|c|c|}
\hline & \multicolumn{2}{|c|}{ In vitro data } & \multicolumn{7}{|c|}{ In vivo data ${ }^{1}$} \\
\hline & 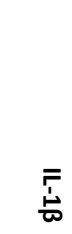 & 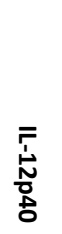 & 䆚 & 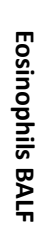 & 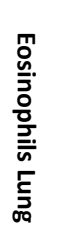 & 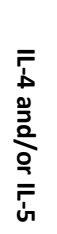 & 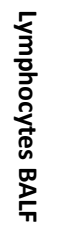 & 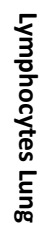 & $\begin{array}{l}\frac{m}{5} \\
\frac{5}{5} \\
\frac{5}{5}\end{array}$ \\
\hline $\mathrm{CeO}_{2}(0 \% \mathrm{Zr})$ & + & - & + & + & + & + & $+/-$ & - & - \\
\hline $\mathrm{CeO}_{2}(27 \% \mathrm{Zr})$ & + & - & + & + & + & + & + & - & - \\
\hline $\mathrm{CeO}_{2}(78 \% \mathrm{Zr})$ & + & - & $+/-$ & + & + & + & + & - & - \\
\hline $\mathrm{Co}_{3} \mathrm{O}_{4}\left(0 \% \mathrm{Fe}_{2} \mathrm{O}_{3}\right)$ & - & + & $+/-$ & - & $+/-$ & - & + & + & + \\
\hline $\mathrm{Co}_{3} \mathrm{O}_{4}\left(25 \% \mathrm{Fe}_{2} \mathrm{O}_{3}\right)$ & - & + & + & - & $+/-$ & $+/-$ & + & + & + \\
\hline $\mathrm{Co}_{3} \mathrm{O}_{4}\left(75 \% \mathrm{Fe}_{2} \mathrm{O}_{3}\right)$ & - & - & $+/-$ & - & $+/-$ & $+/-$ & + & + & + \\
\hline $\mathrm{Co}_{3} \mathrm{O}_{4}\left(0 \% \mathrm{Fe}_{3} \mathrm{O}_{4}\right)$ & - & $+/-$ & $+/-$ & - & - & $+/-$ & + & + & + \\
\hline $\mathrm{Co}_{3} \mathrm{O}_{4}\left(25 \% \mathrm{Fe}_{3} \mathrm{O}_{4}\right)$ & - & - & $+/-$ & - & $+/-$ & $+/-$ & + & + & + \\
\hline $\mathrm{Co}_{3} \mathrm{O}_{4}\left(75 \% \mathrm{Fe}_{3} \mathrm{O}_{4}\right)$ & - & $+/-$ & $+/-$ & - & $+/-$ & $+/-$ & + & + & + \\
\hline
\end{tabular}

${ }^{1}$ Source: Dekkers et al., $2019^{[11]}$. +: clear increase, +/-: moderate increase, -: no increase in the biomarker of effect.

Our in vivo study indicates that the adjuvant properties of $\mathrm{CeO}_{2} \mathrm{NPs}$ are primarily associated with a Th2 type immune response (IL-4, IL-5, IgE and eosinophils in BALF and lung) (Table 3, ${ }^{[11]}$ ). Linking this in vivo response to inflammasome activation by these NPs is not straightforward (Table 3). Inflammasome activation is directly involved in a Th17 type immune response via IL-1 $\beta$ induction and also has a function in Th1 cell development via IL-18 induction. Th17 responses induce the recruitment of neutrophils and macrophages to tissues, while Th1 responses enhance the killing efficacy of macrophages and the proliferation of cytotoxic $T$ cells ${ }^{[13]}$. These Th17 and Th1 responses, do not match with the observed Th2 type responses observed in our in vivo study. However, data from aluminium adjuvants indicate that NLRP3 
inflammasome activation may also enhance Th2 cell responses ${ }^{[15]}$. IL-1 $\beta$ can enhance humoral immunity, while IL-18 has been shown to increase IgE antibody production ${ }^{[15]}$. Although inflammasome induced Th2 cell responses are not as well established as the Th17 and Th1 responses, they would better match with the immune responses observed in vivo (Table 3). The fact that $\mathrm{CeO}_{2}$ NPs exposure of DC did not increase IL-12p40 production (Table 3) may be indicative for a lack of Th1 response, since IL-12p40 is associated with a Th1 type immune response that is related to cellular inflammation ${ }^{[12,19]}$. Taken together, the Th2 type immune responses observed in vivo cannot be readily predicted using the inflammasome activation and DC maturation assays.

The adjuvant activity of the $\mathrm{Co}_{3} \mathrm{O}_{4}$ NPs in our in vivo study was characterised by a less pronounced Th2 type immune response and an increase in lymphocytes in BALF and the development of ectopic lymphoid-like structures (ELS) in the lung tissue. ELS are tertiary lymphoid organs, characterized by well-organized T- and B-cell areas enmeshed with dendritic and stromal cells, usually localized within the perivascular or interstitial areas of the lung ${ }^{[45]}$. ELS formation is associated with lymphoid tissue inducer cells, and the induction of lymphotoxin- $\alpha$, CXCL13, CCL19, and CCL21. However, these mediators are not mandatory for its formation ${ }^{[46]}$. Dendritic cells, however, have been identified as pivotal players required to form and sustain the presence of inducible bronchus-associated lymphoid tissue ${ }^{[46]}$.The observed increase in IL-12p40 production in DC exposed to $\mathrm{Co}_{3} \mathrm{O}_{4} \mathrm{NPs}$ are therefore in line with these observed immune responses in vivo. IL-12 induction triggers the cellular immune system via the differentiation of naïve T cells into Th1 and cytotoxic T lymphocytes. In response to IL-12 signalling, Th1 cells induce IFN- $\gamma$, IL-2, lymphotoxin $\alpha$, and TNF- $\beta$ resulting in the recruitment of macrophages, neutrophils, NK cells, cytotoxic T cells and B cells ${ }^{[47]}$. Although no increase in IFN- $\gamma$ concentrations was found in the BALF, the increase in IL-12p40 production by DC is consistent with the increased numbers of lymphocytes in BALF and lung tissue and the formation of ELS observed in our in vivo study. In our in vitro assay with DCs only IL-12p40 was measured as an indicator of DC maturation. Measuring other biomarkers in the DC assay, including surface markers and other cytokines, may provide further information to link the in vitro to the in vivo results. In a study using the same DC assay, IL-12p40 production was found to have a rather similar potential as CD83 and CD86 expression to discriminate between two crystal structures of $\mathrm{TiO}_{2} \mathrm{NPs}^{[3]}$. The lack of inflammasome activation by $\mathrm{Co}_{3} \mathrm{O}_{4}$ NPs exposure are not in line with the induction of ELS. IL-1 $\beta$ is a known inducer of a Th17 immune response, including IL-17 production, which is known to induce ELS ${ }^{[48]}$. Taken together, although the increase in IL-12p40 may be indicative for the ELS observed in vivo, the observed immune responses in vivo cannot be readily predicted using the inflammasome activation and DC maturation assays. 


\section{Conclusions}

The results from our in vitro studies and previously performed in vivo study indicate that the type of immune response of NPs in in vitro or in vivo models is not related to the percentage of doping, the size, or the acellular redox activity. The chemical composition seems to be more important in determining the type of response. $\mathrm{CeO}_{2} \mathrm{NPs}$ (doped and undoped) but not $\mathrm{Co}_{3} \mathrm{O}_{4} \mathrm{NPs}$ (doped and undoped) induced inflammasome activation. The results from our in vitro studies may indicated some specific immune responses in vivo, such as the potential lymphoid accumulation, which is associated with a pronounced increase in IL-12p40 in DC in vitro. However, more studies with NPs with different chemical compositions are needed to investigate the predictivity of the in vitro DC maturation assay for lymphoid accumulation in vivo. Next to the inflammasome activation and DC maturation assay, additional in vitro assays covering other immune mechanisms that can be influenced by NP exposure are needed to more accurately predict the different types of immune responses underlying the adjuvant activity of NPs in vivo.

\section{Acknowledgement}

The authors would like to thank Daan Leseman of RIVM for his technical support.

\section{Funding}

This work was supported by the Seventh Framework Programme for research, technology development and demonstration of the European Union under Grant Agreement Number 310451 (NanoMILE) and the Netherlands Food and Consumer Product Safety Authority (NVWA, project number V090016). 


\section{References}

1. Boraschi, D.; Swartzwelter, B.J.; Italiani, P. Interaction of engineered nanomaterials with the immune system: Health-related safety and possible benefits. Current Opinion in Toxicology 2018, 10, 74-83, doi:https://doi.org/10.1016/j.cotox.2018.02.002.

2. Meldrum, K.; Guo, C.; Marczylo, E.L.; Gant, T.W.; Smith, R.; Leonard, M.O. Mechanistic insight into the impact of nanomaterials on asthma and allergic airway disease. Part Fibre Toxicol 2017, 14, 45, doi:10.1186/s12989-017-0228-y.

3. Vandebriel, R.J.; Vermeulen, J.P.; van Engelen, L.B.; de Jong, B.; Verhagen, L.M.; de la FonteyneBlankestijn, L.J.; Hoonakker, M.E.; de Jong, W.H. The crystal structure of titanium dioxide nanoparticles influences immune activity in vitro and in vivo. Part Fibre Toxicol 2018, 15, 9, doi:10.1186/s12989-018-0245-5.

4. Boraschi, D.; Italiani, P. From Antigen Delivery System to Adjuvanticy: The Board Application of Nanoparticles in Vaccinology. Vaccines (Basel) 2015, 3, 930-939, doi:10.3390/vaccines3040930.

5. Bakand, S.; Hayes, A. Toxicological Considerations, Toxicity Assessment, and Risk Management of Inhaled Nanoparticles. Int J Mol Sci 2016, 17, doi:10.3390/ijms17060929.

6. Sun, B.; Wang, X.; Ji, Z.; Li, R.; Xia, T. NLRP3 inflammasome activation induced by engineered nanomaterials. Small 2013, 9, 1595-1607, doi:10.1002/smll.201201962.

7. Dayem, A.A.; Kim, B.; Gurunathan, S.; Choi, H.Y.; Yang, G.; Saha, S.K.; Han, D.; Han, J.; Kim, K.; Kim, J.H., et al. Biologically synthesized silver nanoparticles induce neuronal differentiation of SH-SY5Y cells via modulation of reactive oxygen species, phosphatases, and kinase signaling pathways. Biotechnol J 2014, 9, 934-943, doi:10.1002/biot.201300555.

8. Zhang, H.; Ji, Z.; Xia, T.; Meng, H.; Low-Kam, C.; Liu, R.; Pokhrel, S.; Lin, S.; Wang, X.; Liao, Y.P., et al. Use of metal oxide nanoparticle band gap to develop a predictive paradigm for oxidative stress and acute pulmonary inflammation. ACS Nano 2012, 6, 4349-4368, doi:10.1021/nn3010087.

9. Li, R.; Ji, Z.; Chang, C.H.; Dunphy, D.R.; Cai, X.; Meng, H.; Zhang, H.; Sun, B.; Wang, X.; Dong, J., et al. Surface interactions with compartmentalized cellular phosphates explain rare earth oxide nanoparticle hazard and provide opportunities for safer design. ACS Nano 2014, 8, 1771-1783, doi:10.1021/nn406166n.

10. Wang, X.; Sun, B.; Liu, S.; Xia, T. Structure Activity Relationships of Engineered Nanomaterials in inducing NLRP3 Inflammasome Activation and Chronic Lung Fibrosis. Nanolmpact 2017, 6, 99108, doi:10.1016/j.impact.2016.08.002.

11. Dekkers, S.; Wagner, J.G.; Vandebriel, R.J.; Eldridge, E.A.; Tang, S.V.Y.; Miller, M.R.; Römer, I.; de Jong, W.H.; Harkema, J.R.; Cassee, F.R. Role of chemical composition and redox modification of poorly soluble nanomaterials on their ability to enhance allergic airway sensitisation in mice. Particle and Fibre Toxicology 2019, 16, 39, doi:10.1186/s12989-019-0320-6.

12. Ihrie, M.D.; Bonner, J.C. The Toxicology of Engineered Nanomaterials in Asthma. Curr Environ Health Rep 2018, 5, 100-109, doi:10.1007/s40572-018-0181-4.

13. Lee, T.H.; Song, H.J.; Park, C.S. Role of inflammasome activation in development and exacerbation of asthma. Asia Pac Allergy 2014, 4, 187-196, doi:10.5415/apallergy.2014.4.4.187. 
14. Besnard, A.G.; Guillou, N.; Tschopp, J.; Erard, F.; Couillin, I.; Iwakura, Y.; Quesniaux, V.; Ryffel, B.; Togbe, D. NLRP3 inflammasome is required in murine asthma in the absence of aluminum adjuvant. Allergy 2011, 66, 1047-1057, doi:10.1111/j.1398-9995.2011.02586.x.

15. Chen, M.; Wang, H.; Chen, W.; Meng, G. Regulation of adaptive immunity by the NLRP3 inflammasome. Int Immunopharmacol 2011, 11, 549-554, doi:10.1016/j.intimp.2010.11.025.

16. Evavold, C.L.; Kagan, J.C. How Inflammasomes Inform Adaptive Immunity. J Mol Biol 2018, 430, 217-237, doi:10.1016/j.jmb.2017.09.019.

17. Nakanishi, K. Unique Action of Interleukin-18 on T Cells and Other Immune Cells. Front Immunol 2018, 9, 763, doi:10.3389/fimmu.2018.00763.

18. Xu, D.; Trajkovic, V.; Hunter, D.; Leung, B.P.;Schulz, K.; Gracie, J.A.; McInnes, I.B.; Liew, F.Y. IL-18 induces the differentiation of Th1 or Th2 cells depending upon cytokine milieu and genetic background. Eur J Immunol 2000, 30, 3147-3156, doi:10.1002/1521-4141(200011)30:11<3147::AIDIMMU3147>3.0.CO;2-J.

19. Jia, J.; Zhang, Y.; Xin, Y.; Jiang, C.; Yan, B.; Zhai, S. Interactions Between Nanoparticles and Dendritic Cells: From the Perspective of Cancer Immunotherapy. Front Oncol 2018, 8, 404, doi:10.3389/ fonc.2018.00404.

20. Xia, T.; Hamilton, R.F.; Bonner, J.C.; Crandall, E.D.; Elder, A.; Fazlollahi, F.; Girtsman, T.A.; Kim, K.; Mitra, S.; Ntim, S.A., et al. Interlaboratory evaluation of in vitro cytotoxicity and inflammatory responses to engineered nanomaterials: the NIEHS Nano GO Consortium. Environ Health Perspect 2013, 121, 683-690, doi:10.1289/ehp.1306561.

21. Chanput, W.; Mes, J.J.; Wichers, H.J. THP-1 cell line: an in vitro cell model for immune modulation approach. Int Immunopharmacol 2014, 23, 37-45, doi:10.1016/j.intimp.2014.08.002.

22. Hamilton, R.F., Jr.; Xiang, C.; Li, M.; Ka, I.; Yang, F.; Ma, D.; Porter, D.W.; Wu, N.; Holian, A. Purification and sidewall functionalization of multiwalled carbon nanotubes and resulting bioactivity in two macrophage models. Inhal Toxicol 2013, 25, 199-210, doi:10.3109/08958378.2013.775197.

23. Cabanas, A.; Darr, J.A.; E., L.; Poliakoff, M. A continous and clean one-step synthesis of nanoparticulate $\mathrm{Ce}_{1-\mathrm{x}} \mathrm{Zr}_{x} \mathrm{O}_{2}$ solid solutions in near-critical water. Chem Comm 2000, 2000, 901-902.

24. Liu, J.; Römer, I.; Tang, S.V.Y.; Valsami-Jones, E.; Palmer, R.E. Crystallinity depends on choice of iron salt precursor in the continous hydrothermal synthesis of Fe-Co oxide nanoparticles. RSC Adv 2017, 7, 37436-37440, doi:DOI: 10.1039/c7ra06647c.

25. Giannakou, C.; Aimonen, K.; Bloois, L.V.; Catalan, J.; Geertsma, R.E.; Gremmer, E.R.; de Jong, W.H.; Keizers, P.H.; Schwillens, P.L.; Vandebriel, R.J., et al. Sensitive method for endotoxin determination in nanomedicinal product samples. Nanomedicine (Lond) 2019, 14, 1231-1246, doi:10.2217/ nnm-2018-0339.

26. Malyala, P.; Singh, M. Endotoxin limits in formulations for preclinical research. J Pharm Sci 2008, 97, 2041-2044, doi:10.1002/jps.21152.

27. DeLoid, G.M.; Cohen, J.M.; Pyrgiotakis, G.; Demokritou, P. Preparation, characterization, and in vitro dosimetry of dispersed, engineered nanomaterials. Nat Protoc 2017, 12, 355-371, doi:10.1038/nprot.2016.172. 
28. DeLoid, G.M.; Cohen, J.M.; Pyrgiotakis, G.; Pirela, S.V.; Pal, A.; Liu, J.; Srebric, J.; Demokritou, P. Advanced computational modeling for in vitro nanomaterial dosimetry. Part Fibre Toxicol 2015, 12, 32, doi:10.1186/s12989-015-0109-1.

29. Cohen, J.M.; Teeguarden, J.G.; Demokritou, P. An integrated approach for the in vitro dosimetry of engineered nanomaterials. Part Fibre Toxicol 2014, 11, 20, doi:10.1186/1743-8977-11-20.

30. Slob, W. Dose-response modeling of continuous endpoints. Toxicol Sci 2002, 66, 298-312, doi:10.1093/toxsci/66.2.298.

31. Al-Shabib, N.A.; Husain, F.M.; Ahmed, F.; Khan, R.A.; Khan, M.S.; Ansari, F.A.; Alam, M.Z.; Ahmed, M.A.; Khan, M.S.; Baig, M.H., et al. Low Temperature Synthesis of Superparamagnetic Iron Oxide (Fe304) Nanoparticles and Their ROS Mediated Inhibition of Biofilm Formed by Food-Associated Bacteria. Front Microbiol 2018, 9, 2567, doi:10.3389/fmicb.2018.02567.

32. Zhou, R.; Tardivel, A.; Thorens, B.; Choi, I.; Tschopp, J. Thioredoxin-interacting protein links oxidative stress to inflammasome activation. Nat Immunol 2010, 11, 136-140, doi:10.1038/ ni.1831.

33. Hamilton, R.F., Jr.; Wu, Z.; Mitra, S.; Shaw, P.K.; Holian, A. Effect of MWCNT size, carboxylation, and purification on in vitro and in vivo toxicity, inflammation and lung pathology. Part Fibre Toxicol 2013, 10, 57, doi:10.1186/1743-8977-10-57.

34. Ji, Z.; Wang, X.; Zhang, H.; Lin, S.; Meng, H.; Sun, B.; George, S.; Xia, T.; Nel, A.E.; Zink, J.I. Designed synthesis of $\mathrm{CeO} 2$ nanorods and nanowires for studying toxicological effects of high aspect ratio nanomaterials. ACS Nano 2012, 6, 5366-5380, doi:10.1021/nn3012114.

35. Mizutani, N.; Nabe, T.; Yoshino, S. Exposure to multiwalled carbon nanotubes and allergen promotes early- and late-phase increases in airway resistance in mice. Biol Pharm Bull 2012, 35, 2133-2140.

36. Vandebriel, R.J.; Dekkers, S.; de Jong, W.H.; Cassee, F.R. An Update on NLRP3 Inflammasome Activation by Engineered Nanomaterials. Current Bionanotechnology 2016, 2, 40-46.

37. Cho, W.S.; Duffin, R.; Bradley, M.; Megson, I.L.; Macnee, W.; Lee, J.K.; Jeong, J.; Donaldson, K. Predictive value of in vitro assays depends on the mechanism of toxicity of metal oxide nanoparticles. Part Fibre Toxicol 2013, 10, doi:10.1186/1743-8977-10-55.

38. Graham, U.M.; Wang, C.; Fernback, J.; Dozier, A.K.; Drummy, L.; Mahalingam, K.; Molina, R.M.; Konduru, N.V.; Birch, E.; Brain, J.D. In vivo formation of Ce-phosphate Nanoparticles following Intratracheal Instillation of $\mathrm{CeCl} 3$ : Subcellular sites, Nanostructures, Precipitation Mechanisms and Nanoparticle 3D-Alignment. Microsc. Microanal. 2017, 23, 1342-1343, doi:doi:10.1017/ S1431927617007371.

39. Graham, U.M.; Yokel, R.A.; Dozier, A.K.; Drummy, L.; Mahalingam, K.; Tseng, M.T.; Birch, E.; Fernback, J. Analytical High-resolution Electron Microscopy Reveals Organ-specific Nanoceria Bioprocessing. Toxicol Pathol 2018, 46, 47-61, doi:10.1177/0192623317737254.

40. Sager, T.M.; Wolfarth, M.; Leonard, S.S.; Morris, A.M.; Porter, D.W.; Castranova, V.; Holian, A. Role of engineered metal oxide nanoparticle agglomeration in reactive oxygen species generation and cathepsin B release in NLRP3 inflammasome activation and pulmonary toxicity. Inhal Toxicol 2016, 28, 686-697, doi:10.1080/08958378.2016.1257664. 
41. Asati, A.; Santra, S.; Kaittanis, C.; Nath, S.; Perez, J.M. Oxidase-like activity of polymer-coated cerium oxide nanoparticles. Angew Chem Int Ed Engl 2009, 48, 2308-2312, doi:10.1002/ anie.200805279.

42. Mirshafiee, V.; Sun, B.; Chang, C.H.; Liao, Y.P.; Jiang, W.; Jiang, J.; Liu, X.; Wang, X.; Xia, T.; Nel, A.E. Toxicological Profiling of Metal Oxide Nanoparticles in Liver Context Reveals Pyroptosis in Kupffer Cells and Macrophages versus Apoptosis in Hepatocytes. ACS Nano 2018, 12, 3836-3852, doi:10.1021/acsnano.8b01086.

43. Dankers, A.C.A.; Kuper, C.F.; Boumeester, A.J.; Fabriek, B.O.; Kooter, I.M.; Grollers-Mulderij, M.; Tromp, P.; Nelissen, I.; Zondervan-Van Den Beuken, E.K.; Vandebriel, R.J. A practical approach to assess inhalation toxicity of metal oxide nanoparticles in vitro. J Appl Toxicol 2018, 38, 160-171, doi:10.1002/jat.3518.

44. Schanen, B.C.; Das, S.; Reilly, C.M.; Warren, W.L.; Self, W.T.; Seal, S.; Drake, D.R., 3rd. Immunomodulation and $\mathrm{T}$ helper $\mathrm{TH}(1) / \mathrm{TH}(2)$ response polarization by $\mathrm{CeO}(2)$ and $\mathrm{TiO}(2)$ nanoparticles. PLoS One 2013, 8, e62816, doi:10.1371/journal.pone.0062816.

45. Marin, N.D.; Dunlap, M.D.; Kaushal, D.; Khader, S.A. Friend or Foe: The Protective and Pathological Roles of Inducible Bronchus-Associated Lymphoid Tissue in Pulmonary Diseases. J Immunol 2019, 202, 2519-2526, doi:10.4049/jimmunol.1801135.

46. Foo, S.Y.; Phipps, S. Regulation of inducible BALT formation and contribution to immunity and pathology. Mucosal Immunol 2010, 3, 537-544, doi:10.1038/mi.2010.52.

47. Kumar, S.; Jeong, Y.; Ashraf, M.U.; Bae, Y.S. Dendritic Cell-Mediated Th2 Immunity and Immune Disorders. Int J Mol Sci 2019, 20, doi:10.3390/ijms20092159.

48. Jones, G.W.; Hill, D.G.; Jones, S.A. Understanding Immune Cells in Tertiary Lymphoid Organ Development: It Is All Starting to Come Together. Front Immunol 2016, 7, 401, doi:10.3389/ fimmu.2016.00401. 


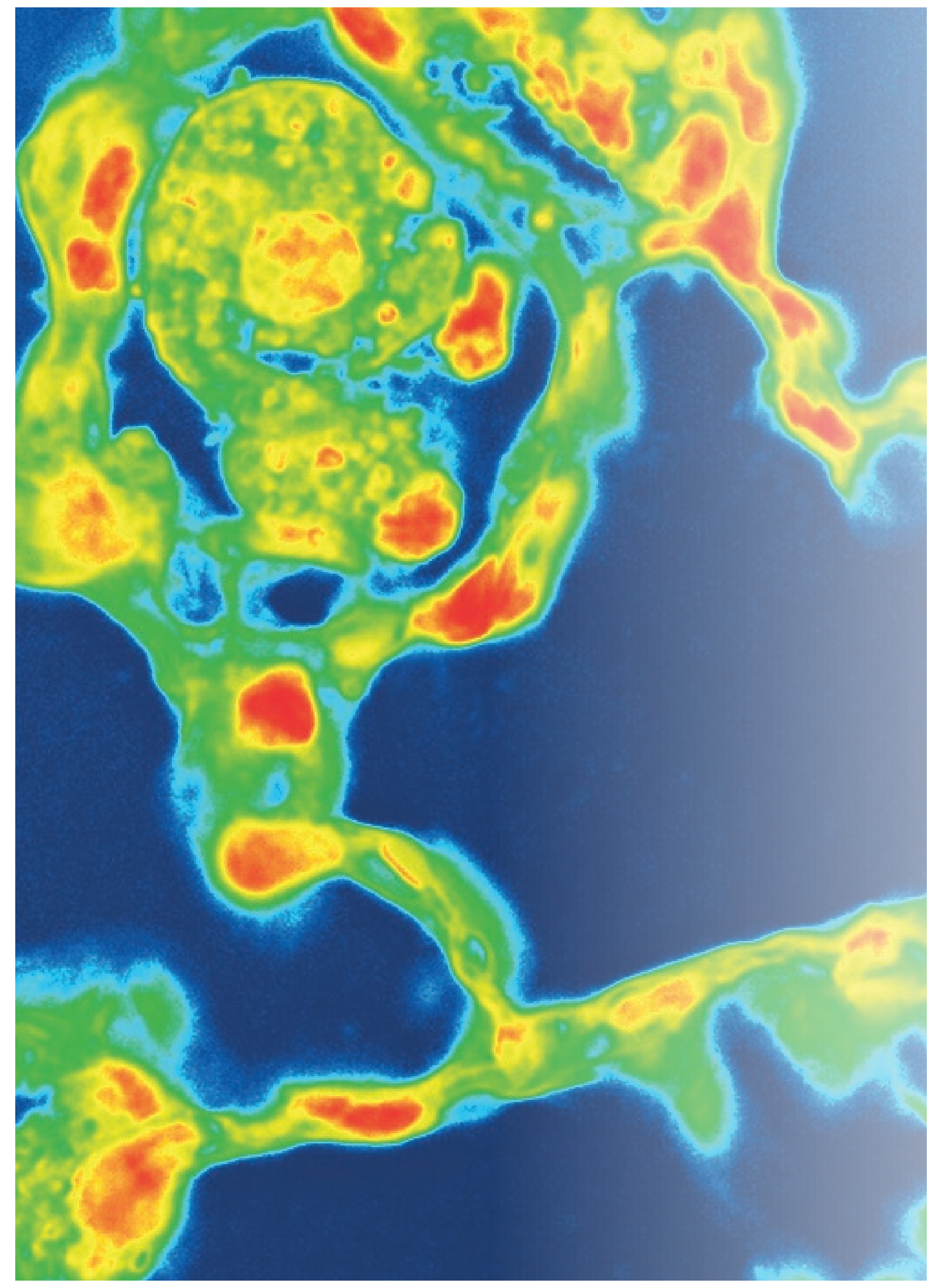




\section{Chapter 7}

General Discussion 


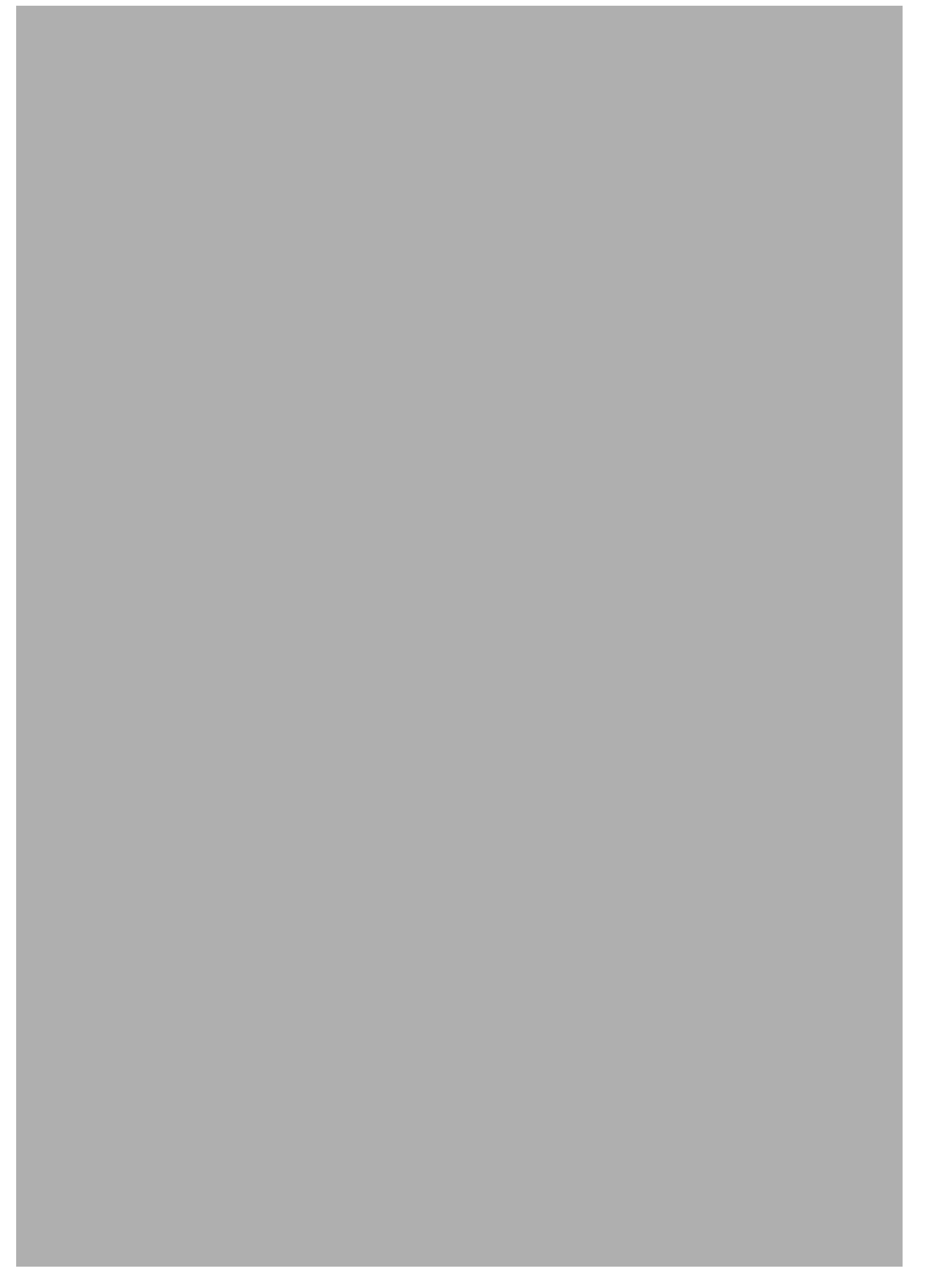




\section{Introduction}

The research described in this thesis investigated the influence of several physicochemical properties of nanoparticles (NPs) on their biodistribution, molecular pathways and toxicity after inhalation. Investigating the influence of physicochemical properties of NPs on their potential to cause toxicological effects is more complicated then it seems, because the physicochemical properties of NPs may change due to interactions of the NPs with their surrounding environment within a toxicity assay. In this chapter, the key findings, overall conclusions, future perspectives and implications of this research are discussed.

\section{Inhalation toxicity in vivo}

After four weeks of exposure to undoped and $\mathrm{Zr}$-doped $\mathrm{CeO}_{2} \mathrm{NPs}$ via inhalation, no major toxicological effects were observed in mice, apart from minimal inflammatory histopathology in the lung (chapter 2). This was unexpected, since previous inhalation studies in rats and mice showed more pronounced effects after exposure to $\mathrm{CeO}_{2} \mathrm{NPs}$ (Aalapati et al., 2014; Arts et al., 2016; Gosens et al., 2014; Keller et al., 2014). From a toxicological point of view, this was a bit disappointing, since it is more difficult to determine the influence of redox activity on the toxicity if only minimal effects are observed. From the perspective of risk assessment, this was an interesting observation that was important to publish, knowing that studies with clear toxicological effects are generally more represented in literature than studies with negative results (Mlinaric et al., 2017). A detailed comparison of data on the undoped $\mathrm{CeO}_{2} \mathrm{NP}$ from our study with data from other inhalation studies with various $\mathrm{CeO}_{2}$ NPs showed that the difference in the pulmonary responses to $\mathrm{CeO}_{2} \mathrm{NPs}$ can be explained by differences in the tested species and size, but also on other physicochemical characteristics of the particles (chapter 3 ). No relation between the redox activity of the $\mathrm{CeO}_{2} \mathrm{NPs}$, as measured using the acellular Ferric Reduction Ability of Serum (FRAS) assay and the observed toxicological responses was found. However, since the various $\mathrm{CeO}_{2} \mathrm{NPs}_{\text {in }}$ the analyzed studies differed in more than one physicochemical characteristic, it was difficult to determine the influence of each individual characteristic on the observed toxicity.

Within our inhalation study, we used a structural series of $\mathrm{CeO}_{2} \mathrm{NPs}$ that only differed with respect to the amount of $\mathrm{Zr}$-doping. Besides redox activity, the $\mathrm{Zr}$-doping also changed the chemical composition, but most of the other physicochemical characteristics, such as primary particles size, aggregation and agglomeration were similar. The redox modification via Zrdoping had limited effects on the observed pulmonary toxicity after subchronic inhalation of $\mathrm{CeO}_{2}$ NPs. However, because only minimal toxicological pulmonary effects were observed after exposure of to the undoped $\mathrm{CeO}_{2} \mathrm{NPs}$, most of the hypothesized ameliorative effects resulting from the $\mathrm{ZrO}_{2}$-doping may not have been visible in this study. Another factor that makes investigating the influence of redox activity difficult, is that the redox activity of NPs is not only dependent on physicochemical properties such as chemical composition, size, 
surface area and surface chemistry, but also on the environmental conditions which the NPs encounter in the in vivo study, including changes in $\mathrm{pH}$ and the presence of proteins and salts. There are many different assays to measure the redox activity or the subsequent chemical and biological responses. A combination of different assays and environmental conditions is needed to investigate the influence of the redox activity of a NP on the outcome of a toxicological assay. In preparation of our four week inhalation study, the redox activity was measured with Electron Paramagnetic Resonance (EPR) under several acellular conditions. Additional information on the redox activity of the NPs under the environmental conditions relevant for the in vivo inhalation study, e.g. in lung lining, lysosomal fluid, or cellular conditions, may have provided more insight why $\mathrm{Zr}$-doping of $\mathrm{CeO}_{2} \mathrm{NPs}$ did not influence the toxicological responses observed in the inhalation study. Maybe $\mathrm{Zr}$-doping of $\mathrm{CeO}_{2}$ NPs does not lead to differences in the ability of these NPs to generate or reduce reactive oxygen species (ROS) under the environmental conditions of the in vivo inhalation study, because of modification or transformation of the NPs. Maybe other mechanisms of toxicity are more important in the inhalation toxicity than the hypothesized redox activity, ROS generation, oxidative stress and subsequent inflammation.

\section{Molecular mechanisms of in vitro toxicity}

Further investigation into the molecular pathways of the undoped and $\mathrm{Zr}$-doped $\mathrm{CeO}_{2} \mathrm{NPs}$ was performed within our in vitro multi-omics study (chapter 4). Surprisingly, exposure to the same $\mathrm{Zr}$-doped $\mathrm{CeO}_{2} \mathrm{NPs}$ showed remarkably less cytotoxicity compared to the previous in vitro study in the same cell type (A549) (chapter 2). Furthermore, only minor molecular responses were observed, which indicated that the influence of redox activity on the assumed oxidative stress mechanistic pathway of $\mathrm{CeO}_{2} \mathrm{NP}$ toxicity was very limited. Similar to the in vivo inhalation study, additional information on the redox activity of the NPs under the environmental conditions relevant for the in vitro assay, e.g. in cell culture medium, lysosomal fluid, or cellular conditions, may have provided more insight why Zr-doping of $\mathrm{CeO}_{2}$ NPs did not influence the toxicological responses observed in this in vitro study.

In contrast to these somewhat discouraging results for the undoped and $\mathrm{Zr}$-doped $\mathrm{CeO}_{2}$ NPs within our in vitro multi-omics study, the results for the Ag and ZnO NPs confirmed our hypothesis that dissolution and the release of $\mathrm{Ag}$ and $\mathrm{Zn}$ ions are the main drivers of the in vitro toxicity $\mathrm{Ag}$ and $\mathrm{ZnO}$ NPs. The majority of molecular responses of A549 cells to the $\mathrm{Ag}$ and $\mathrm{ZnO}$ NPs, were similar to their responses to $\mathrm{Ag}$ and $\mathrm{Zn}$ ions, respectively, confirming previous findings that the main toxicological pathways of $\mathrm{Ag}$ and $\mathrm{ZnO}$ NPs were mediated by dissolved metal ions rather than by the physical aspects of the NPs (De Matteis et al., 2015; Liu et al., 2016). This study shows the advantage of designing a toxicity assay in such a way that the influence of time dependent effects and environmental conditions can be taken into account. Including ionic controls in the study design, made it possible to compare the molecular responses of the NPs plus their released ions to those of the ions alone. 
Measuring the molecular responses over time, made it possible to follow the development and reversibility or persistence of the observed molecular responses. Dissolution testing in cell culture medium provided additional information on the influence of relevant environmental conditions on the dissolution of the Ag and $\mathrm{ZnO}$ NPs.

\section{Adjuvant activity in vivo}

The ability of NPs to generate ROS has not only been related to inflammatory pulmonary responses, but also to cellular responses associated with the ability of NPs to influence allergic airways sensitization (Meldrum et al., 2017). As Zr-doping of $\mathrm{CeO}_{2}$ NPs had limited effect on the inflammatory responses after inhalation, additional NPs were included in our in vivo study investigating the influence of redox activity and chemical composition on the adjuvant activity on NPs. Fe-doped $\mathrm{Co}_{3} \mathrm{O}_{4}$ NPs were included as they were expected to have a greater ability to induce oxygen radicals, oxidative stress, pulmonary inflammation and adjuvant activity. Similar to the results from the four week inhalation study and the in vitro assays in A549 cells, again no relation between the acellular redox activity of the undoped and $\mathrm{Zr}$-doped $\mathrm{CeO}_{2} \mathrm{NPs}$, and the observed toxicological responses were found. Also for the undoped and Fe-doped $\mathrm{Co}_{3} \mathrm{O}_{4}$ NPs the influence of the redox activity on the toxicological responses was very limited.

However, the adjuvant potency of the different NPs varied with the different immune responses and indicated that different immunological pathways are involved in the adjuvant activity of undoped and $\mathrm{Zr}$-doped $\mathrm{CeO}_{2} \mathrm{NPs}$ compared to undoped and Fe-doped $\mathrm{Co}_{3} \mathrm{O}_{4}$ NPs. These differences in immune responses suggest that chemical composition has more influence on the type of allergic immune responses than the redox activity of NPs. The most interesting observations were the lymphoid cell accumulation and formation of ectopic lymphoid tissues in the lungs after co-exposure to OVA and undoped or Fe-doped $\mathrm{Co}_{3} \mathrm{O}_{4}$ NPs. Although, broncho-associated lymphoid tissue has been associated with chronic allergic diseases, such as asthma, the pathways that control the development and function of ectopic or broncho-associated lymphoid tissues are poorly understood (Hirahara et al., 2018; Hwang et al., 2016). More knowledge on the underlying mechanistic pathways, their interrelationships and control mechanisms are needed to better understand the influence of various physicochemical properties on the adjuvant activity of NPs.

\section{Inflammasome activation and dendritic cell maturation in vitro}

To gain more insight into the different immunological responses involved in the adjuvant activity of undoped and $\mathrm{Zr}$-doped $\mathrm{CeO}_{2} \mathrm{NPs}$ compared to undoped and Fe-doped $\mathrm{Co}_{3} \mathrm{O}_{4} \mathrm{NPs}$, the same NPs were tested in two in vitro assays as model for interaction of NPs and cells of the immune system. These in vitro assays investigate two mechanisms known to play an important role in NP-enhanced induction of allergic airway responses (Ihrie and Bonner, 2018; Meldrum et al., 2017), inflammasome activation and dendritic cell maturation. Similar 
to the in vivo study on adjuvant activity, the outcomes of the in vitro studies indicated that inflammasome activation and dendritic cell maturation is primarily related to the chemical composition and not to the size or acellular redox activity of undoped and $\mathrm{Zr}$ doped $\mathrm{CeO}_{2}$ NPs or undoped and Fe-doped $\mathrm{Co}_{3} \mathrm{O}_{4}$ NPs. Undoped and $\mathrm{Zr}$-doped $\mathrm{CeO}_{2}$ NPs induced inflammasome activation, while several undoped and Fe-doped $\mathrm{Co}_{3} \mathrm{O}_{4} \mathrm{NPs}$ induced dendritic cell maturation. The ranking of the various NPs with respect to their in vitro IL-12p40 response was similar to the ranking of their in vivo B-cell density in the lungs, indicating that the results of the DC maturation assay may be indicative for the potential of NPs to induce in vivo lymphoid accumulation in lungs. However, more studies measuring not only IL-12p40, but also other cytokines, chemokines and surface makers after exposure to a larger variety of NPs are needed to further investigate the mechanisms of action and to verify the predictive value of this assay for lymphoid accumulation in the lungs. Moreover, the type of immune responses observed in vivo cannot be readily predicted using in vitro assays on DC maturation and inflammasome activation alone, since these assays only cover two of the key events in the AOP network on respiratory allergic responses.

\section{Overall conclusions}

Redox modification through Zr-doping of $\mathrm{CeO}_{2} \mathrm{NPs}$ and Fe-doping of $\mathrm{Co}_{3} \mathrm{O}_{4} \mathrm{NPs}$ did not decreased the in vitro and in vivo toxicity and adjuvant activity of these NPs. The influence of the redox activity of these NPs on the investigated biological responses was very limited and did not follow a consequent trend of either an increase or a decrease with increasing redox activity. Comparison of the results of our inhalation study with other inhalation studies suggested that species, size and other physicochemical properties of the NPs are more important determinants of the inflammatory responses observed after inhalation of $\mathrm{CeO}_{2}$ NPs than the redox activity of the NPs. The results of our in vitro multi-omics study indicated that chemical composition and dissolution were more important drivers of the molecular responses observed in $\mathrm{A} 549$ cells than redox activity. After exposure to $\mathrm{CeO}_{2}$ NPs, only minor molecular responses were observed, while exposure to Ag and ZnO NPs resulted in more pronounced molecular responses that were mediated by dissolved metal ions rather than by the physical aspects of the NPs. The results of the in vivo study on the adjuvant activity and the in vitro studies on inflammasome activation and DC maturation indicated that chemical composition has more influence on the type of immune responses than the redox activity of NPs. Although the role of redox activity in the toxicity of NPs cannot be ruled out, its influence on the various biological responses investigated in this thesis was limited and not very clear, as this property strongly depends on the environmental conditions of the toxicological assays, which is difficult to measure, simulate or predict. When investigating the influence of the physicochemical properties of NPs on the biological response, it is important to assess how these properties may change over time depending on their surrounding environment. Although full characterization of the NPs throughout 
the whole assay may not always be possible, it is always important to take the potential influence of the experimental conditions on the NP properties and subsequent outcomes of the assay into account.

\section{Future perspectives and implications}

Working with nanomaterials within toxicological assays is more complicated then it seems. To investigate the influence of physicochemical properties of NPs on their potential to cause toxicological effects structural series of NPs that only differed with respect to one physicochemical property are needed. This is challenging because changing one property usually influences at least one, but often several other properties. This makes it difficult to separate the effect of one property from that of the other properties. Therefore it is very important to measure how changes in the physicochemical property of interest may also change other physicochemical properties. In addition, physicochemical properties of NPs may change over time due to interaction of the NPs with their surrounding environment. Consequently, knowledge on changes within physicochemical properties of the NPs under the environmental conditions relevant for the toxicological assay is needed to evaluate the influence of the different physicochemical properties on the outcome of the toxicological assay. Furthermore, variabilities in the toxicity assays used to investigate the potential toxicological effects of NPs often hampers comparisons between adverse effects of NPs with different characteristics across different studies. Therefore, more systematic research and harmonization or standardization of the various in vitro and in vivo assays to evaluate the potential toxicity of NPs is needed.

The research described in this thesis highlights the importance of not only the intrinsic physicochemical properties of NPs, but also the changes in these properties due to changes in their surrounding environment, the (extrinsic) physicochemical properties. Although, many researchers have stressed the importance of good physicochemical characterization of NMs within nanotoxicological research, a lot of scientific papers only include the physicochemical characteristics of the NMs as manufactured, but lack detailed analysis of the physicochemical properties under the changing environmental conditions of the toxicological assay (Gao and Lowry, 2018). Transformations, such as dissolution, changes in surface chemistry (including protein coating of NPs in the biological environment i.e. corona formation), aggregation and agglomeration are of great influence on the deposition, uptake, distribution and potential toxicity of the NPs. Although it is not always possible to measure these transformations and changes in physicochemical properties throughout the whole toxicity assay, it is important to know which physicochemical properties may change throughout each assay and how this may influence the outcome of the assay. Within many nanotoxicological research projects access to the right expertise and technical equipment for NM characterization is obtained via collaboration with material and analytical scientists. 
It is recommended to fully utilize this collaboration to enable the characterization of the NMs under the environmental conditions relevant for the toxicological assays of interest.

Insight into which conditions change which physicochemical properties of NPs and how this influences their biodistribution, mechanistic pathways and toxicity will support further development of the various approaches to assess and predict the toxicity of NPs. This will facilitate the selection or verification of the physicochemical properties, functional assays and toxicity assays included in the various risk assessment approaches, Integrated Approaches to Testing and Assessment (IATAs), risk assessment tools, grouping, read across approaches and predictive models. Furthermore it will help to determine the experimental conditions and the experimental design of the analytical, functional and toxicity assays.

Another challenge when investigating the influence of physicochemical properties on the biological responses of NPs is that there is usually more than one mechanistic pathway by which NPs can cause a specific biological response. Moreover, the body generally also has ways to maintain or repair the homeostasis, leading to a complex situation of connected AOP network. Therefore, it is important to know how the various mechanisms of toxicity by which NPs may cause a specific biological response are connected to each other. Immunological responses are, for example, regulated via various mechanistic pathways, which are often connected to each other. It is therefore difficult if not impossible to predict all potential adverse effects in vivo based on in vitro assays. Currently, there are several Adverse Outcome Pathways (AOPs) developed and under development. Omics studies can help to identify the various mechanisms of toxicity of different NMs under different circumstances within these AOPs. Depending on the chemical composition, other NP properties, route of exposure, expected changes or transformations of the NPs and knowledge on the mechanisms by which this NPs may cause adverse effects, a different set of acellular (functional) assays and cellular in vitro assays is needed to predict the toxicity in vivo. However, it cannot be expected that such a set of functional assays and in vitro assays will result in a perfect prediction of the in vivo toxicity, since this is currently not even possible for non-nanomaterials.

The research in this thesis provides more insight into some of the environmental conditions that influence some of the physicochemical properties and how this may influence various biological responses after exposure to NPs in the lung. The challenge for future research is to fill the remaining knowledge gaps on how the various intrinsic properties, extrinsic properties and mechanistic pathways are connected to each other. 


\section{References}

Aalapati S, Ganapathy S, Manapuram S, Anumolu G and Prakya BM (2014) Toxicity and bio-accumulation of inhaled cerium oxide nanoparticles in CD1 mice. Nanotoxicology 8:786-798.

Arts JH, Irfan MA, Keene AM, Kreiling R, Lyon D, Maier M, Michel K, Neubauer N, Petry T, Sauer UG, Warheit D, Wiench K, Wohlleben W and Landsiedel R (2016) Case studies putting the decisionmaking framework for the grouping and testing of nanomaterials (DF4nanoGrouping) into practice. Regul Toxicol Pharmacol 76:234-261.

De Matteis V, Malvindi MA, Galeone A, Brunetti V, De Luca E, Kote S, Kshirsagar P, Sabella S, Bardi G and Pompa PP (2015) Negligible particle-specific toxicity mechanism of silver nanoparticles: the role of $\mathrm{Ag}+$ ion release in the cytosol. Nanomedicine 11:731-739.

Gao X and Lowry GV (2018) Progress towards standardized and validated characterizations for measuring physicochemical properties of manufactured nanomaterials relevant to nano health and safety risks. Nanolmpact 9:14-30.

Gosens I, Mathijssen LE, Bokkers BG, Muijser H and Cassee FR (2014) Comparative hazard identification of nano- and micro-sized cerium oxide particles based on 28-day inhalation studies in rats. Nanotoxicology 8:643-653.

Hirahara K, Shinoda K, Endo Y, Ichikawa T and Nakayama T (2018) Maintenance of memory-type pathogenic Th2 cells in the pathophysiology of chronic airway inflammation. Inflamm Regen 38:10.

Hwang JY, Randall TD and Silva-Sanchez A (2016) Inducible Bronchus-Associated Lymphoid Tissue: Taming Inflammation in the Lung. Front Immunol 7:258.

Ihrie MD and Bonner JC (2018) The Toxicology of Engineered Nanomaterials in Asthma. Curr Environ Health Rep 5:100-109.

Keller J, Wohlleben W, Ma-Hock L, Strauss V, Gröters S, Küttler K, Wiench K, Herden C, Oberdörster G, Ravenzwaay B and Landsiedel R (2014) Time course of lung retention and toxicity of inhaled particles: short-term exposure to nano-Ceria. Arch Toxicol 88.

Liu J, Feng X, Wei L, Chen L, Song B and Shao L (2016) The toxicology of ion-shedding zinc oxide nanoparticles. Crit Rev Toxicol 46:348-384.

Meldrum K, Guo C, Marczylo EL, Gant TW, Smith R and Leonard MO (2017) Mechanistic insight into the impact of nanomaterials on asthma and allergic airway disease. Part Fibre Toxicol 14:45.

Mlinaric A, Horvat M and Supak Smolcic V (2017) Dealing with the positive publication bias: Why you should really publish your negative results. Biochem Med (Zagreb) 27:030201. 


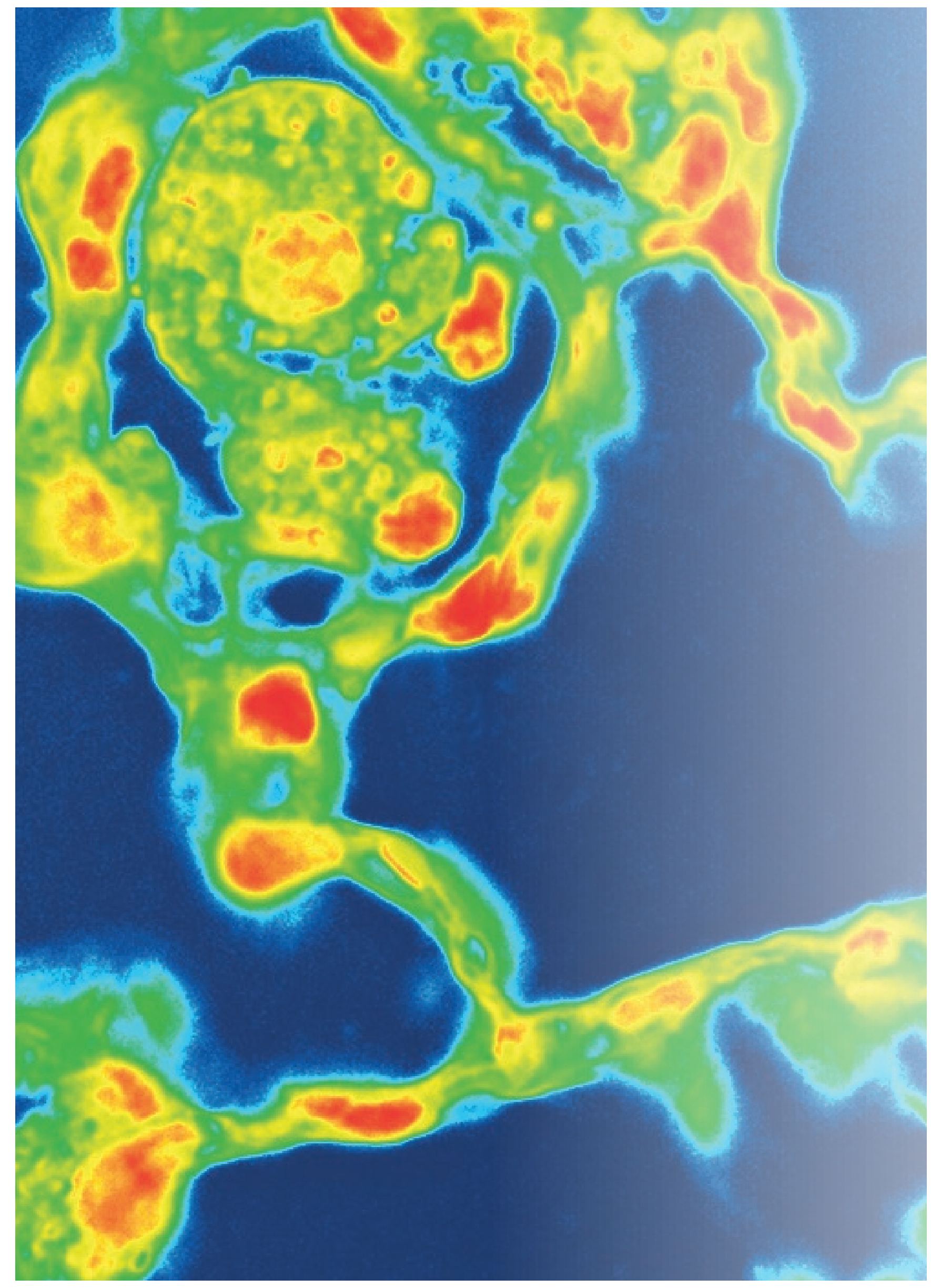


Summary

Samenvatting

Curriculum vitea

List of publications

Dankwoord 


\section{Summary}

Nanotechnology makes it possible to develop products with amazing features. By changing the various properties of nanomaterials it is possible to develop materials with remarkable functionalities. However, it is often not known how changing the properties may affect the ability of nanomaterials to cause adverse human health effects. More knowledge in the influence of the various physicochemical properties on the potential human health effects will allow the development of nanomaterials with amazing features without introducing increased human health risks.

The main aim of the research described in this thesis is to investigate how the physicochemical properties of nanomaterials influence assumed pathways of toxicity after inhalation. This was done using a carefully selected range of in vivo and in vitro toxicity assays in combination with a carefully selected set nanoparticles (NPs) with specific properties that were synthesized within the European NanoMILE project. This research aim and approach can be translated into the following research objectives:

1. Assess the influence of size and redox activity of undoped and zirconium(Zr)-doped cerium dioxide $\left(\mathrm{CeO}_{2}\right)$ NPs on their deposition, biodistribution and pulmonary (and cardiovascular) effects in mice and rats following inhalation (chapter 2 and 3 ) .

2. Investigate the influence of dissolution and redox activity of silver (Ag), zinc oxide ( $\mathrm{ZnO}$ ) and undoped and $\mathrm{Zr}$-doped $\mathrm{CeO}_{2}$ NPs on the molecular mechanisms by which these NPs induce toxicity in A549 cells in vitro (chapter 4).

3. Evaluate the influence of chemical composition and redox activity of undoped and Zrdoped $\mathrm{CeO}_{2}$ and undoped and iron(Fe)-doped cobalt oxide $\left(\mathrm{Co}_{3} \mathrm{O}_{4}\right)$ NPs on their adjuvant properties in vivo (chapter 5 ).

4. Study the influence of chemical composition and redox activity of undoped and Zrdoped $\mathrm{CeO}_{2}$ and undoped and Fe-doped $\mathrm{Co}_{3} \mathrm{O}_{4}$ NPs on in vitro inflammasome activation and dendritic cell maturation (chapter 6$)$.

The central hypothesis was that redox modification (changing the tendency to acquire or lose electrons) through $\mathrm{Zr}$-doping of $\mathrm{CeO}_{2}$ and Fe-doping of $\mathrm{CO}_{3} \mathrm{O}_{4} \mathrm{NPs}$ would lead to a decrease in the ability of the NPs to generate Reactive Oxygen Species (ROS), oxidative stress and subsequent in vitro and in vivo toxicity and in vivo adjuvant activity. Furthermore, it is expected that the pathways of toxicity of Ag and ZnO NPs are related to their dissolution and ability to release metal ions.

\section{In vivo biodistribution and toxicity after inhalation}

The influence of redox activity of $\mathrm{CeO}_{2} \mathrm{NPs}$ on the biodistribution, pulmonary and cardiovascular effects after inhalation was investigated by exposure of mice to $\mathrm{CeO}_{2} \mathrm{NPs}$ 
with varying amounts of $\mathrm{Zr}$-doping ( $0 \%, 27 \%$ or $78 \% \mathrm{Zr}$ ) (chapter 2$)$. Healthy mice (C57BL/6J), mice prone to cardiovascular disease ( $\mathrm{ApoE}^{-/}$, western-diet fed) and a mouse model of neurological disease (5xFAD) were exposed via nose-only inhalation over a four-week period ( $4 \mathrm{mg} / \mathrm{m}^{3}$ for $3 \mathrm{~h} /$ day, 5 days/week). Effects were assessed four weeks post-exposure.

In all three mouse models exposure to $4 \mathrm{mg} \mathrm{CeO}_{2} \mathrm{NP} / \mathrm{m}^{3}$ had no major toxicological effects apart from some modest inflammatory responses in the lung, which were not related to the amount of $\mathrm{Zr}$-doping. In $\mathrm{ApoE}^{-/}$mice $\mathrm{CeO}_{2} \mathrm{NP}$ exposure did not change the size of atherosclerotic plaques in the brachiocephalic artery compared to non-exposed animals, but there was a trend towards increased inflammatory cell content in the plaques in relation to the $\mathrm{Zr}$ content of the $\mathrm{CeO}_{2} \mathrm{NPs}$. These findings indicate that redox modification of $\mathrm{CeO}_{2}$ NPs via Zr-doping has limited effects on the pulmonary and cardiovascular toxicity after subchronic exposure. However, because only minimal effects were observed after exposure to the $\mathrm{CeO}_{2}$ NPs without $\mathrm{Zr}$-doping, the hypothesized ameliorative effects resulting from the Zr-doping may not have been visible in this study.

Indeed in comparison to other inhalation studies with $\mathrm{CeO}_{2} \mathrm{NPs}$, the observed toxicological effects in our study were relatively mild. A detailed analysis of the data from several inhalation studies with different exposure durations, species and $\mathrm{CeO}_{2}$ nanoforms, indicated that the differences in the observed toxicity between these studies can be explained by rats being generally more susceptible to the effects of exposure to particles compared to mice and by differences in kinetics of the NPs (chapter 3). However, differences in species and kinetics could not fully explain the differences in observed toxicity in the analysed studies, indicating that pulmonary responses do not only depend on the species and NP properties that influence the kinetics, such as particle size, but also on other physicochemical characteristics of the NPs.

\section{Molecular mechanisms of in vitro toxicity}

The influence of redox modification of $\mathrm{CeO}_{2} \mathrm{NPs}$ via $\mathrm{Zr}$-doping was further explored by investigating the molecular pathways by which undoped and $\mathrm{Zr}$-doped $\mathrm{CeO}_{2} \mathrm{NPs}$ induce in vitro toxicity in a multi-omics study (chapter 4). Next to the influence of redox activity of $\mathrm{Zr}$-doped $\mathrm{CeO}_{2} \mathrm{NPs}$, also the influence of the dissolution or ability to release metal ions on the molecular pathways of in vitro toxicity of $\mathrm{Ag}$ and $\mathrm{ZnO}$ NPs was investigated in this study. Human lung epithelial cells ( $\mathrm{A} 549$ ) were exposed to various $\mathrm{Ag}, \mathrm{ZnO}$, and $\mathrm{CeO}_{2} \mathrm{NPs}, \mathrm{Ag}$ and $\mathrm{ZnO}$ micro-sized particles (MPs), $\mathrm{Ag}$ ions $\left(\mathrm{Ag}^{+}\right)$and zinc ions $\left(\mathrm{Zn}^{2+}\right)$ over a $24 \mathrm{~h}$ time course. Molecular responses at exposure levels that caused $\sim 20 \%$ cytotoxicity were characterised by direct infusion mass spectrometry lipidomics and polar metabolomics and by RNAseq transcriptomics. 
Exposure to undoped and $\mathrm{Zr}$-doped $\mathrm{CeO}_{2}$ NPs showed remarkably less cytotoxicity compared to the previous in vitro study using the same NPs in the same cell type (A549) (chapter 2). Furthermore, $\mathrm{CeO}_{2} \mathrm{NPs}$ elicited few molecular changes, showing slight evidence of oxidative stress for only one of the four $\mathrm{CeO}_{2}$ NPs tested, indicating that the influence of redox activity on the assumed oxidative stress mechanistic pathway of $\mathrm{CeO}_{2} \mathrm{NP}$ toxicity was very limited.

All $\mathrm{Ag}, \mathrm{Zn}$ and $\mathrm{ZnO}$ exposures resulted in significant metabolic and transcriptional responses. The majority of these molecular changes were common to both ionic and NP exposures and characteristic of metal ion exposure, confirming that the modes of action of these NPs are largely mediated by dissolved metal ions rather than by the physical aspects of the NPs. The molecular changes were time dependent, which can be explained by differences in uptake rates and dissolution kinetics resulting in different intracellular concentrations or different intracellular compartmentalization of the metal ions. The time dependency of the molecular changes may also be explained by adaptive changes, such as induction of metallothioneins that ameliorate cellular damage by sequestering the metal ions.

\section{Adjuvant activity in vivo}

The influence of redox activity on the adjuvant activity of NPs was investigated in a mouse model of airway sensitization (chapter 5). As Zr-doping of $\mathrm{CeO}_{2} \mathrm{NPs}$ had limited effect on the inflammatory responses after inhalation, additional NPs were included in this in vivo study. Fe-doped $\mathrm{Co}_{3} \mathrm{O}_{4}$ NPs were included as they were expected to have a greater ability to induce oxygen radicals, oxidative stress, pulmonary inflammation and adjuvant activity. Co-exposure of BALB/c mice to ovalbumin (OVA) and all tested NPs resulted in enhanced immune responses to OVA. The responses to co-exposure of OVA and the various NPs were not affected by the amount of doping or the level of acellular redox activity of the NPs, but the type of immune response was affected chemical composition of the NPs. The cytokine profiles showed that co-exposure to OVA and undoped or $\mathrm{Zr}$-doped $\mathrm{CeO}_{2} \mathrm{NPs}$ primarily induced Th2 type responses, while co-exposure to OVA and undoped or Fe-doped $\mathrm{Co}_{3} \mathrm{O}_{4}$ NPS induced no or less marked increases in IgE plasma levels, BALF IL-4 and IL-5 concentrations and percentages of eosinophils in BALF, but more pronounced increases in BALF IL-6 concentrations and percentages of lymphocytes in BALF. In addition, co-exposure to OVA and $\mathrm{Co}_{3} \mathrm{O}_{4} \mathrm{NPs}$, but not $\mathrm{CeO}_{2} \mathrm{NPs}$, induced lymphoid cell accumulation and ectopic lymphoid structures (ELS) in the lungs, which are normally absent in mice, but can be induced by inflammatory processes and infections.

\section{In vitro inflammasome activation and dendritic cell maturation}

In vitro exposure of THP-1 macrophages to various NPs indicated that undoped and Zrdoped $\mathrm{CeO}_{2}$ NPs induced inflammasome activation, while undoped and Fe-doped $\mathrm{Co}_{3} \mathrm{O}_{4} \mathrm{NPS}$ did not (chapter 6 ). Inflammasomes are protein complexes that can be formed and activated in macrophages in reaction to various stimuli, such as bacteria, viruses, asbestos or NPs. 
Inflammasome activation leads to the production of pro-inflammatory cytokines, including as IL-1 $\beta$.

Undoped $\mathrm{Co}_{3} \mathrm{O}_{4}$ NPs increased interleukine-12p40 (IL-12p40) production by dendritic cells, indicative for dendritic cell maturation, while undoped and $\mathrm{Zr}$-doped $\mathrm{CeO}_{2} \mathrm{NPs}$ did not. Dendritic cell maturation is the process by which dendritic cells are further evolved or developed to enable antigen presentation and activation of T-cells. Doping effects of $\mathrm{Co}_{3} \mathrm{O}_{4}$ NPs on the IL-12p40 production were mixed.

Similar to the results of our in vivo study (chapter 5), the results of these in vitro assays indicate that the type of immune response of the NPs is primarily related to the chemical composition and not to the particle size or acellular redox activity of the NPs. Furthermore, a pronounced increase in IL-12p40 in dendritic cells in vitro seemed to be indicative for lymphoid accumulation in lung tissue in vivo. However, additional studies with NPs with different chemical compositions and in vitro assays covering other mechanisms are needed to more accurately predict the adjuvant activity of NPs in vivo.

\section{Conclusions and future perspectives}

Redox modification through Zr-doping of $\mathrm{CeO}_{2} \mathrm{NPs}$ and Fe-doping of $\mathrm{CO}_{3} \mathrm{O}_{4} \mathrm{NPs}$ did not decrease the in vitro and in vivo toxicity and adjuvant activity of these NPs. The influence of the redox activity of these NPs on the investigated biological responses was very limited and did not follow a consequent trend of either an increase or a decrease with increasing redox activity. However, investigating the influence of redox activity on biological responses is complicated, as changing the redox activity of NPs usually also changes other properties, such as chemical composition. This makes it difficult to separate the effect of redox activity from that of the other NP properties. Furthermore, the redox activity of NPs may change over time due to interaction of the NPs with their surrounding environment. The environmental conditions, including $\mathrm{pH}$ and the presence of proteins and salt, are different in the various in vitro and in vivo toxicity assays and may change over time throughout the assay. Therefore, a combination of different assays and environmental conditions is needed to investigate the influence of the redox activity of a NP on the outcome of a toxicological assay. The choice of these assays and environmental conditions should be based on the chemical composition of the NP under investigation and the specific environmental conditions the NP is expected to encounter in the toxicity assay.

Although the role of redox activity in the toxicity of NPs cannot be ruled out, the results of the in vitro and in vivo assays described in the this thesis indicate that other physicochemical properties of the tested NPs, including particle size, dissolution and chemical composition, are more important determinants of the investigated biological responses. Also for particle size, dissolution and chemical composition it is important to assess how they may change 
over time depending on their surrounding environment. The results of our in vitro multiomic study demonstrate the advantage of designing a toxicity assay in such a way that the influence of environmental conditions over time can be taken into account. By including ionic controls in the study design, measuring the molecular responses over time, and performing dissolution testing in cell culture medium, it was possible to investigate the influence of relevant environmental conditions on the dissolution of the Ag and ZnO NPs and the subsequent development and reversibility or persistence of the observed molecular responses. This shows that knowledge on changes within physicochemical properties of the NPs under the environmental conditions relevant for the toxicological assay is needed to evaluate the influence of the different physicochemical properties on the outcome of the toxicological assay. Consequently, variabilities in environmental conditions within the various toxicity assays used to investigate the potential toxicological effects of NPs often hampers comparisons between adverse effects of NPs with different characteristics across different studies. Hence, more systematic research and harmonization or standardization of the various in vitro and in vivo assays to evaluate the potential toxicity of NPs is needed.

Another challenge when investigating the influence of physicochemical properties on the biological responses of NPs is that there is usually more than one mechanistic pathway by which NPs can cause a specific biological response. This was demonstrated by the results of the in vivo study on the adjuvant activity and the in vitro studies on inflammasome activation and dendritic cell maturation. The adjuvant potency of the different NPs in vivo varied with the different immune responses and indicated that different immunological pathways are involved in the adjuvant activity of undoped and $\mathrm{Zr}$-doped $\mathrm{CeO}_{2} \mathrm{NPs}$ compared to undoped and Fe-doped $\mathrm{Co}_{3} \mathrm{O}_{4}$ NPs. Lymphoid cell accumulation and formation of ectopic lymphoid tissues in the lungs only occurred after co-exposure to OVA and undoped or Fedoped $\mathrm{Co}_{3} \mathrm{O}_{4} \mathrm{NPs}$. The outcomes of the in vitro studies indicated that undoped and $\mathrm{Zr}$-doped $\mathrm{CeO}_{2} \mathrm{NPs}$ induced inflammasome activation, while several undoped and Fe-doped $\mathrm{Co}_{3} \mathrm{O}_{4}$ NPs induced dendritic cell maturation. The ranking of the various NPs with respect to their in vitro IL-12p40 response was similar to the ranking of their in vivo B-cell density in the lungs, indicating that the results of the dendritic cell maturation assay may be indicative for the potential of NPs to induce in vivo ectopic lymphoid tissues in lungs. However, the type of immune responses observed in vivo cannot be readily predicted using in vitro assays on dendritic cell maturation and inflammasome activation alone, since these assays only cover two of the key events in the AOP network on respiratory allergic responses.

The results of the research presented in this thesis provides more insight how some physicochemical properties and environmental conditions may influence various biological responses after exposure to NPs in the lung. The challenge for future research is to fill the remaining knowledge gaps on how the various physicochemical properties, environmental conditions and underlying mechanistic pathways are connected to each other. 


\section{Samenvatting}

Nanotechnologie maakt het mogelijk om producten te ontwikkelen met uitzonderlijke kwaliteiten. Door de verschillende eigenschappen van nanomaterialen (NMs) te veranderen is het mogelijk om materialen te ontwikkelen met buitengewoon aantrekkelijke functionaliteiten. $\mathrm{Er}$ is nog veel onduidelijk over hoe deze veranderingen onze gezondheid mogelijk nadelig kunnen beïnvloeden. Meer kennis over de invloed van de verschillende fysisch-chemische eigenschappen op de mogelijke gezondheidseffecten zal het mogelijk maken om aantrekkelijke NMs te ontwikkelen zonder de risico's voor de gezondheid te vergroten.

Het belangrijkste doel van het onderzoek dat in dit proefschrift wordt beschreven is om te onderzoeken hoe de fysisch-chemische eigenschappen van NMs van invloed kunnen zijn op de veronderstelde toxicologische werkingsmechanismen van NMs na inhalatie. Hiervoor werd een zorgvuldig geselecteerde reeks in vivo en in vitro toxiciteitstesten gebruikt in combinatie met een zorgvuldig geselecteerde set van binnen het Europese NanoMILEproject gesynthetiseerd NMs met specifieke eigenschappen. Het doel en de aanpak van het onderzoek kunnen worden vertaald in de volgende onderzoeksdoelstellingen:

1. Het bepalen van de invloed van deeltjesgrootte en redoxactiviteit van ongedopeerde en zirkonium(Zr)gedopeerde ceriumdioxide $\left(\mathrm{CeO}_{2}\right) \mathrm{NMs}$ op de long depositie, biodistributie en pulmonaire (en cardiovasculaire) effecten in muizen en ratten na inhalatie (hoofdstuk 2 en 3).

2. Het onderzoeken van de invloed van oplosbaarheid en redoxactiviteit van zilver (Ag), zinkoxide $(\mathrm{ZnO})$ en ongedopeerde en $\mathrm{Zr}$-gedopeerde $\mathrm{CeO}_{2} \mathrm{NMs}$ op de moleculaire mechanismen waarmee deze NMs in vitro toxiciteit induceren in A549-cellen (hoofdstuk 4).

3. Het evalueren van de invloed van de chemische samenstelling en redoxactiviteit van ongedopeerde en $\mathrm{Zr}$-gedopeerde $\mathrm{CeO}_{2}$ en ongedopeerde en ijzer(Fe)-gedopeerde kobaltoxide $\left(\mathrm{Co}_{3} \mathrm{O}_{4}\right) \mathrm{NMs}$ op de adjuverende werking in vivo (hoofdstuk 5).

4. Het bestuderen van de invloed van de chemische samenstelling en redoxactiviteit van ongedopeerde en $\mathrm{Zr}$-gedopeerde $\mathrm{CeO}_{2}$ en ongedopeerde en Fe-gedopeerde $\mathrm{Co}_{3} \mathrm{O}_{4} \mathrm{NMs}$ op in vitro inflammasoom activatie en dendritische cel maturatie (hoofdstuk 6).

De centrale hypothese was dat redoxmodificatie (het veranderen van de mate waarin de NMs kunnen oxideren of reduceren) door $\mathrm{Zr}$-dopering van $\mathrm{CeO}_{2}$ en Fe-dopering van $\mathrm{Co}_{3} \mathrm{O}_{4} \mathrm{NMs}$ zou leiden tot een afname van het vermogen van de NMs om reactieve zuurstofradicalen, oxidatieve stress en daaropvolgende in vitro en in vivo toxiciteit en in vivo adjuvantie te veroorzaken. Daarnaast wordt verwacht dat de toxicologische werkingsmechanismen van 
Ag en ZnO NMs gerelateerd zijn aan hun oplosbaarheid en het vermogen om metaalionen af te geven.

\section{In vivo biodistributie en toxiciteit na inademing}

De invloed van redoxactiviteit van $\mathrm{CeO}_{2} \mathrm{NMs}$ op de biodistributie, pulmonaire en

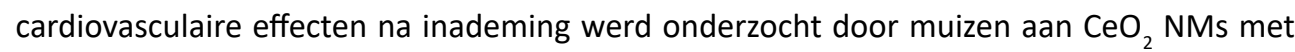
wisselende hoeveelheden $\mathrm{Zr}$-dopering $(0 \%, 27 \%$ of $78 \% \mathrm{Zr}$ ) bloot te stellen (hoofdstuk 2). Gezonde muizen ( $\mathrm{C} 57 \mathrm{BL} / 6)$, muizen die vatbaar zijn voor hart- en vaatziekten (ApoE ${ }^{-1}$ , westerse voeding) en een muismodel van neurologische aandoeningen (5xFAD) werden gedurende een periode van vier weken blootgesteld via "nose only" inhalatie $\left(4 \mathrm{mg} / \mathrm{m}^{3}\right.$ gedurende 3 uur/dag, 5 dagen/week). De effecten werden vier weken na blootstelling bepaald.

In alle drie de muismodellen veroorzaakte blootstelling aan $4 \mathrm{mg} \mathrm{CeO} \mathrm{NMs}_{2} / \mathrm{m}^{3}$ alleen minimale toxicologische effecten, waaronder enkele geringe ontstekingsreacties in de longen die niet gerelateerd waren aan de hoeveelheid $\mathrm{Zr}$-dopering. In $\mathrm{ApoE}^{-/-}$muizen veranderde $\mathrm{CeO}_{2} \mathrm{NM}$-blootstelling de grootte van atherosclerotische plaques ten opzichte van niet blootgestelde dieren niet, maar was er wel een trend zichtbaar waarbij een verhoogd $\mathrm{Zr}$-gehalte van de $\mathrm{CeO}_{2} \mathrm{NMs}$ tot een verhoogd gehalte aan ontstekingscellen in de plaques in de truncus branchiocephalicus leidde. Deze bevindingen geven aan dat redoxmodificatie van $\mathrm{CeO}_{2} \mathrm{NMs}$ via $\mathrm{Zr}$-dopering weinig invloed heeft op de pulmonaire en cardiovasculaire toxiciteit na subchronische blootstelling. Omdat echter slechts minimale effecten werden waargenomen na blootstelling aan $4 \mathrm{mg} / \mathrm{m}^{3} \mathrm{CeO}_{2} \mathrm{NMs}$ zonder $\mathrm{Zr}$-dopering, was de veronderstelde vermindering van de toxiciteit als gevolg van de $\mathrm{Zr}$-dopering mogelijk niet zichtbaar in deze studie.

In vergelijking met andere inhalatiestudies met $\mathrm{CeO}_{2} \mathrm{NMs}$ waren de waargenomen toxicologische effecten in onze studie inderdaad relatief mild. Een gedetailleerde analyse van de gegevens van verschillende inhalatiestudies met verschillende blootstellingsduur, diersoorten en typen $\mathrm{CeO}_{2} \mathrm{NMs}$, gaf aan dat de verschillen in de waargenomen toxiciteit tussen deze studies verklaard kunnen worden doordat ratten over het algemeen gevoeliger zijn voor de effecten van blootstelling aan deeltjes dan muizen en door verschillen in kinetiek van de NMs (hoofdstuk 3). Verschillen in diersoort en kinetiek konden de verschillen in waargenomen toxiciteit in de geanalyseerde onderzoeken echter niet volledig verklaren, wat erop wijst dat pulmonaire reacties niet alleen afhangen van de diersoort en NMeigenschappen die de kinetiek beïnvloeden, zoals de deeltjesgrootte, maar ook van andere fysisch-chemische eigenschappen van de NMs. 


\section{Moleculaire mechanismen van in vitro toxiciteit}

De invloed van redoxmodificatie van $\mathrm{CeO}_{2} \mathrm{NMs}$ via $\mathrm{Zr}$-dopering werd verder onderzocht door de moleculaire routes te onderzoeken waarmee ongedopeerde en Zr-gedopeerde $\mathrm{CeO}_{2} \mathrm{NMs}$ in vitro toxiciteit induceren in een multi-omics studie (hoofdstuk 4). Naast de invloed van redoxactiviteit van ongedopeerde en $\mathrm{Zr}$-gedopeerde $\mathrm{CeO}_{2} \mathrm{NMs}$, werd in deze studie ook de invloed van de oplosbaarheid of het vermogen om metaalionen af te geven op de moleculaire mechanismen van in vitro toxiciteit van $\mathrm{Ag}$ en $\mathrm{ZnO}$ NMs onderzocht. Humane longepitheel cellen (A549) werden gedurende 24 uur blootgesteld aan verschillende $\mathrm{Ag}$, $\mathrm{ZnO}$ en $\mathrm{CeO}_{2} \mathrm{NMs}$, Ag en $\mathrm{ZnO}$ microdeeltjes, $\mathrm{Ag}$ ionen $\left(\mathrm{Ag}^{+}\right)$en zink ionen $\left(\mathrm{Zn}^{2+}\right)$. Moleculaire responses werden gekarakteriseerd bij blootstellingsniveaus die $\sim 20 \%$ cytotoxiciteit veroorzaakten. Lipidomics en polaire metabolomics analyses werden uitgevoerd met behulp van directe infusie massaspectrometrie (DIMS) en transcriptomics analyses met behulp van RNA sequencing.

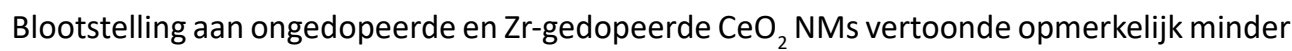
cytotoxiciteit in vergelijking met de vorige in vitro studie met dezelfde NMs in hetzelfde celtype (A549) (hoofdstuk 2). Bovendien veroorzaakten $\mathrm{CeO}_{2} \mathrm{NMs}$ weinig moleculaire veranderingen, welke slechts een gering bewijs vormde voor het veroorzaken van oxidatieve stress voor slechts één van de vier geteste $\mathrm{CeO}_{2} \mathrm{NMs}$. Deze resultaten geven aan dat de invloed van redoxactiviteit op het veronderstelde toxicologische werkingsmechanisme van oxidatieve stress van $\mathrm{CeO}_{2} \mathrm{NMs}$ zeer beperkt was.

Alle blootstellingen aan $\mathrm{Ag}$, Zn en $\mathrm{ZnO}$ deeltjes en ionen resulteerden in significante metabole en transcriptionele responses. De meerderheid van deze moleculaire veranderingen waren kenmerkend voor blootstelling aan metaalionen en werden zowel na blootstelling aan ionen als aan NMs gevonden wat bevestigt dat de werkingsmechanismen van deze NMs grotendeels worden bepaald door opgeloste metaalionen in plaats van door de fysieke aspecten van de NMs. De moleculaire veranderingen waren tijdsafhankelijk, wat verklaard kan worden door verschillen in de opname- en oplossingssnelheden, waardoor verschillen in de concentraties van de metaalionen in de cel en de verschillende compartimenten van de cel ontstaan. De tijdsafhankelijkheid van de moleculaire veranderingen kan ook worden verklaard door adaptieve veranderingen, zoals inductie van metallothioneïnen die cellulaire schade verminderen door de metaalionen te binden.

\section{Adjuverende werking in vivo}

De invloed van redoxactiviteit op de adjuverende werking van NMs werd onderzocht in een muismodel voor allergische astma (hoofdstuk 5). Aangezien $\mathrm{Zr}$-dopering van $\mathrm{CeO}_{2} \mathrm{NMs}$ een beperkt effect had op de ontstekingsreacties na inhalatie, werden additionele NMs in deze in vivo studie opgenomen. Hiervoor werden Fe-gedopeerde $\mathrm{Co}_{3} \mathrm{O}_{4} \mathrm{NMs}$ geselecteerd omdat verwacht werd dat deze NMs een groter vermogen zouden hebben om zuurstofradicalen, 
oxidatieve stress, ontstekingsreacties en adjuverende activiteit te induceren. Gelijktijdige blootstelling van BALB/c-muizen aan ovalbumine (OVA) en alle geteste NMs resulteerde in verhoogde immuunresponses als reactie op blootstelling aan OVA. De responses op blootstelling aan OVA in combinatie met verschillende NMs werden niet beïnvloed door de hoeveelheid dopering of de acellulaire redoxactiviteit van de NMs. Wel werd het type immuunrespons beïnvloed door de chemische samenstelling van de NMs. De cytokineprofielen toonden aan dat gelijktijdige blootstelling aan OVA en ongedopeerde of Zr-gedopeerde $\mathrm{CeO}_{2} \mathrm{NMs}$ voornamelijk Th2-type responses induceerde. Gelijktijdige blootstelling aan OVA en ongedopeerde of Fe-gedopeerde $\mathrm{Co}_{3} \mathrm{O}_{4} \mathrm{NMs}$ induceerde geen of minder duidelijke verhogingen van IgE-plasmaspiegels, BALF IL-4- en IL-5-concentraties en percentages eosinofielen in BALF, maar wel een meer uitgesproken stijging in de BALF IL-6concentratie en het percentage lymfocyten in BALF. Daarnaast induceerde co-blootstelling aan OVA en $\mathrm{Co}_{3} \mathrm{O}_{4} \mathrm{NMs}$ in tegenstelling tot $\mathrm{CeO}_{2} \mathrm{NMs}$, ook accumulatie van lymfoïde cellen en ectopische lymfoïde structuren (ELS) in de longen, welke onder normale omstandigheden afwezig zijn bij muizen, maar geïnduceerd kunnen worden door ontstekingsprocessen en infecties.

\section{In vitro inflammasoom activatie en dendritische cel maturatie}

De resultaten van de in vitro testen waarin THP-1-macrofagen werden blootgesteld aan verschillende $\mathrm{NMs}$ gaven aan dat ongedopeerde en $\mathrm{Zr}$-gedopeerde $\mathrm{CeO}_{2} \mathrm{NMs} \mathrm{inflammasoom}$ activatie induceerden, terwijl ongedopeerde en Fe-gedopeerde $\mathrm{Co}_{3} \mathrm{O}_{4} \mathrm{NMs}$ dat niet deden (hoofdstuk 6). Een inflammasoom is een complex van eiwitten dat in macrofagen gevormd en geactiveerd kan worden in reactie op verschillende stimuli, zoals bacteriën, virussen, asbest of NMs. De activatie van een inflammasoom leidt tot de productie van pro-inflammatoire cytokines, waaronder IL-1 $\beta$.

Niet-gedopeerde $\mathrm{Co}_{3} \mathrm{O}_{4}-\mathrm{NMs}$ verhoogden de productie van interleukine-12p40 (IL$12 \mathrm{p} 40$ ) door dendritische cellen, wat indicatief is voor dendritische cel maturatie, terwijl ongedopeerde en $\mathrm{Zr}$-gedopeerde $\mathrm{CeO}_{2}-\mathrm{NMs}$ dat niet deden. Maturatie van dendritische cellen is de rijping van dendritische cellen, waardoor antigen presentatie en activatie van T-cellen plaats kan vinden. Het effect van Fe-dopering van $\mathrm{Co}_{3} \mathrm{O}_{4}-\mathrm{NMs}$ op de IL-12p40productie was niet eenduidig.

Vergelijkbaar met de resultaten van onze in vivo studie (hoofdstuk 5), geven de resultaten van deze in vitro testen aan dat het type immuunrespons van de NMs primair gerelateerd is aan de chemische samenstelling en niet aan de deeltjesgrootte of de acellulaire redoxactiviteit van de NMs. Daarnaast leek een uitgesproken toename van IL-12p40 in dendritische cellen in vitro indicatief te zijn voor in vivo accumulatie van lymfoïde cellen in de longen. Er zijn echter aanvullende studies met NMs van verschillende chemische samenstellingen en in 
vitro testen die andere mechanismen omvatten nodig om de adjuverende werking van NMs in vivo nauwkeuriger te kunnen voorspellen.

\section{Conclusies en toekomstperspectieven}

Redoxmodificatie van $\mathrm{CeO}_{2} \mathrm{NMs}$ door $\mathrm{Zr}$-dopering en van $\mathrm{Co}_{3} \mathrm{O}_{4} \mathrm{NMs}$ door Fe-dopering verminderde de in vitro en in vivo toxiciteit en adjuverende werking deze NMs niet. De invloed van de redoxactiviteit van deze NMs op de onderzochte biologische processen was zeer beperkt en volgde geen consequente trend door toe- of af te nemen naarmate de redoxactiviteit toe nam. Het onderzoeken van de invloed van redoxactiviteit op biologische processen is echter gecompliceerd, aangezien het veranderen van de redoxactiviteit van NMs meestal ook andere eigenschappen verandert, zoals de chemische samenstelling. Dit maakt het moeilijk om het effect van redoxactiviteit te scheiden van dat van de andere NMeigenschappen. Bovendien kan de redoxactiviteit van NMs in de loop van de tijd veranderen als gevolg van de interactie van de NMs met hun omgeving. De omgevingscondities, zoals de $\mathrm{pH}$ en de aanwezigheid van eiwitten en zouten, zijn verschillend in de verschillende in vitro en in vivo toxiciteitstesten en kunnen in het verloop van een test veranderen. Daarom is een combinatie van verschillende testen en omgevingsomstandigheden nodig om de invloed van de redoxactiviteit van een $\mathrm{NM}$ op de uitkomst van een toxiciteitstest te onderzoeken. De keuze van deze testen en omgevingscondities moet gebaseerd zijn op de chemische samenstelling van het onderzochte NM en de specifieke omgevingscondities die het NM naar verwachting zal tegenkomen in de toxiciteitstesten.

Hoewel niet kan worden uitgesloten dat redoxactiviteit een rol speelt in de toxiciteit van NMs, geven de resultaten van de in vitro en in vivo testen die in dit proefschrift worden beschreven aan dat andere eigenschappen van de geteste NMs, waaronder deeltjesgrootte, oplosbaarheid en chemische samenstelling, belangrijkere determinanten van de onderzochte biologische processen zijn. Ook voor deeltjesgrootte, oplosbaarheid en chemische samenstelling is het belangrijk om te beoordelen hoe deze eigenschappen in de loop van de tijd kunnen veranderen, afhankelijk van hun omgeving. De resultaten van de in vitro multi-omic studie (hoofdstuk 4) tonen het nut aan van een studieopzet waarbij rekening is gehouden met de invloed van omgevingsfactoren in de tijd. Door ionen controles mee te nemen, de moleculaire responses in de tijd te meten en oplosbaarheidstesten uit te voeren in celkweekmedium, was het mogelijk om de invloed van relevante omgevingscondities op de oplosbaarheid van de $\mathrm{Ag}$ en $\mathrm{ZnO}$ NMs en de daaropvolgende ontwikkeling en reversibiliteit van de waargenomen moleculaire responses te onderzoeken. Dit voorbeeld laat zien dat kennis over veranderingen in eigenschappen van de NMs onder voor de toxiciteitstest relevant omgevingscondities nodig is om de invloed van de verschillende fysisch-chemische eigenschappen op de uitkomst van de toxiciteitstest te evalueren. Nu worden vergelijkingen van de nadelige effecten van NMs met verschillende eigenschappen tussen verschillende onderzoeken vaak worden belemmerd door verschillen in de omgevingscondities die in de 
verschillende toxiciteitstesten worden gebruikt. Daarom is meer systematisch onderzoek en harmonisatie of standaardisatie van de verschillende in vitro en in vivo testen nodig voor de evaluatie van de potentiële toxiciteit van NMs.

Een andere uitdaging bij het onderzoeken van de invloed van fysisch-chemische eigenschappen van NMs op de biologische reacties is dat er meestal meer dan één toxicologisch werkingsmechanisme is waarmee NMs een specifieke biologische reactie kunnen veroorzaken. Dit werd gedemonstreerd door de resultaten van de in vivo studie naar de adjuverende werking en de in vitro studies naar inflammasoom activatie en dendritische cel maturatie. De adjuverende activiteit van de verschillende NMs in vivo varieerde voor de verschillende immuunresponses wat aan geeft dat verschillende immunologische processen betrokken zijn bij de adjuverende werking van ongedopeerde en $\mathrm{Zr}$-gedopeerde $\mathrm{CeO}_{2} \mathrm{NMs}$ in vergelijking met ongedopeerde en Fe-gedopeerde $\mathrm{Co}_{3} \mathrm{O}_{4} \mathrm{NMs}$. Accumulatie van lymfoïde cellen en vorming van ectopisch lymfoïde weefsels in de longen trad alleen op na gelijktijdige blootstelling aan OVA en ongedopeerde of Fe-gedopeerde $\mathrm{Co}_{3} \mathrm{O}_{4} \mathrm{NMs}$. De resultaten van de in vitro studies gaven aan dat ongedopeerde en Zr-gedopeerde $\mathrm{CeO}_{2} \mathrm{NMs}$ inflammasoom activatie induceerden, terwijl verschillende ongedopeerde en Fe-gedopeerde $\mathrm{CO}_{3} \mathrm{O}_{4} \mathrm{NMs}$ dendritische cel maturatie induceerden. De ranking van de verschillende NMs met betrekking tot hun in vitro IL-12p40-respons was vergelijkbaar met de ranking van hun in vivo B-celdichtheid in de longen, wat aangeeft dat de resultaten van de dendritische cel maturatie test indicatief kunnen zijn voor de potentie van NMs om in vivo ectopisch lymfoïde weefsels in de longen te induceren. Het type immuunrespons dat in vivo wordt waargenomen kan echter niet gemakkelijk worden voorspeld met behulp van alleen in vitro testen voor inflammasoom activatie en dendritische cel maturatie, aangezien deze testen slechts twee belangrijke werkingsmechanismen in het AOP-netwerk voor respiratoire allergische reacties omvatten.

De uitkomsten van het onderzoek die in dit proefschrift worden beschreven, geven meer inzicht in hoe een aantal fysisch-chemische eigenschappen en omgevingsfactoren verschillende biologische reacties van NMs kunnen beïnvloeden. De uitdaging voor toekomstig onderzoek is om de resterende kennishiaten over hoe de verschillende fysisch-chemische eigenschappen en omgevingscondities elkaar en de onderliggende werkingsmechanismen kunnen beïnvloeden op te vullen. 


\section{Curriculum vitea}

Susan Dekkers was born on May $5^{\text {th }} 1975$ in Utrecht. After graduating from her secondary school (VWO), she attended a one year exchange programme at a High School in the USA. In 1994 she started studying Health Sciences at the Maastricht University and received her BSc degree in 1995, followed by her MSc degree in Environmental Health Sciences in 2000. Before starting her PhD research, Susan worked for several years as a toxicological risk assessor first at TNO and later at RIVM. Within her job at RIVM she was given the opportunity to perform her PhD research. In January 2021, Susan returned to TNO to work on the development and application of innovative and in silico methods in the toxicological risk assessment.

\section{List of publications}

Gimeno-Benito I, Guisti A, Dekkers S, Haase A, Janer G. A review to support the derivation of a worst-case dermal penetration value for nanoparticles. Regul Toxicol Pharmacol. 2021 Feb;119:104836. doi: 10.1016/j.yrtph.2020.104836.

Dekkers S, Wijnhoven SWP, Braakhuis HM, Soeteman-Hernandez LG, Sips AJAM, Tavernaro I, Kraegeloh A, Noorlander CW. Safe-by-Design part I: Proposal for nanospecific human health safety aspects needed along the innovation process. Nanolmpact. 2020; 18: 100227. doi. org/10.1016/j.impact.2020.100227.

Wahle T, Sofranko A, Dekkers S, Miller MR, Heusinkveld HJ, Albrecht C, Cassee FR, Schins RPF. Evaluation of neurological effects of cerium dioxide nanoparticles doped with different amounts of zirconium following inhalation exposure in mouse models of Alzheimer's and vascular disease. Neurochem Int. 2020 Sep;138:104755. doi:10.1016/j.neuint.2020.104755.

Stone V, Stefania Gottardo S, Bleeker EAJ, Braakhuis H, Dekkers S, Fernandes T, Haase A, Hunt $N$, Hristozov D, Jantunen $P$, Jeliazkova $N$, Johnston $H$, Lamon L, Murphy $F$, Rasmussen $K$, Rauscher $H$, Sánchez Jiménez A, Svendsen C, Spurgeon D, Vázquez-Campos S, Wohlleben W, Oomen AG. A framework for grouping and read-across of nanomaterials- supporting innovation and risk assessment. Nano Today. 2020; 35: 100941. doi.org/10.1016/j. nantod.2020.100941.

Nymark P, Bakker M, Dekkers S, Franken R, Fransman W, García-Bilbao A, Greco D, Gulumian M, Hadrup N, Halappanavar S, Hongisto V, Hougaard KS, Jensen KA, Kohonen P, Koivisto AJ, Dal Maso M, Oosterwijk T, Poikkimäki M, Rodriguez-Llopis I, Stierum R, Sørli JB, Grafström R. Toward Rigorous Materials Production: New Approach Methodologies Have Extensive Potential to Improve Current Safety Assessment Practices. Small. 2020 Feb;16(6):e1904749. doi: 10.1002/smll.201904749. 
Soeteman-Hernández LG, Blab GA, Carattino A, Dekker F, Dekkers S, van der Linden M, van Silfhout $A$, Noorlander CW. Challenges of implementing nano-specific safety and safe-by-design principles in academia. Nanolmpact. 2020; 19, 100243. doi.org/10.1016/j. impact.2020.100243.

Dekkers S, Wagner JG, Vandebriel RJ, Eldridge EA, Tang SVY, Miller MR, Römer I, de Jong WH, Harkema JR, Cassee FR. Role of chemical composition and redox modification of poorly soluble nanomaterials on their ability to enhance allergic airway sensitisation in mice. Part Fibre Toxicol. 2019 Oct 28;16(1):39. doi: 10.1186/s12989-019-0320-6.

Soeteman-Hernandez LG, Apostolova MD, Bekker C, Dekkers S, Grafström RC, Groenewold M, Handzhiyski Y, Herbeck-Engel P, Hoehener K, Karagkiozaki V, Kelly S, Kraegeloh A, Logothetidis S, Micheletti C, Nymark P, Oomen AG, Oosterwijk T, Rodríguez-Lopis I, Sabella S, Sanchez Jiménez A, Sips AJAM, Suarez- Merino B, Tavernaro I, van Engelen J, Wijnhoven SWP, Noorlander CW. Safe Innovation Approach: Towards an agile system for dealing with innovations. Materials Today Communications. 2019; 20:100548. doi: 10.1016/j. mtcomm.2019.100548.

Oomen AG, Steinhäuser KG, Bleeker EAJ, van Broekhuizen F, Sips A, Dekkers S, Wijnhoven SWP, Sayre PG. Risk assessment frameworks for nanomaterials: Scope, link to regulations, applicability, and outline for future directions in view of needed increase in efficiency. Nanolmpact. 2018; 9: 1-13. doi.org/10.1016/j.impact.2017.09.001.

Dekkers S, Ma-Hock L, Lynch I, Russ M, Miller MR, Schins RPF, Keller J, Römer I, Küttler K, Strauss V, De Jong WH, Landsiedel R, Cassee FR, Differences in the Toxicity of Cerium Dioxide Nanomaterials after Inhalation can be explained by Lung Deposition. Animal Species and Nanoforms. Inhalation Toxicology, 2018; 30:7-8, 273-286, doi: 10.1080/08958378.2018.1516834.

Yang Y, Xu L, Dekkers S, Zhang LG, Cassee FR, Zuo YY. Aggregation State of Metal-Based Nanomaterials at the Pulmonary Surfactant Film Determines Biophysical Inhibition. Environ Sci Technol. 2018 Aug 7;52(15):8920-8929. doi: 10.1021/acs.est.8b02976.

Dekkers S, Williams TD, Zhang J, Zhou J, Vandebriel RJ, De La Fonteyne LJJ, Gremmer ER, He S, Guggenheim EJ, Lynch I, Cassee FR, De Jong WH, Viant MR. Multi-omics approaches confirm metal ions mediate the main toxicological pathways of metal-bearing nanoparticles in lung epithelial A549 cells. Environ. Sci.: Nano, 2018; 5, 1506. doi: 10.1039/c8en00071a.

Dekkers S, Miller MR, Schins RPF, Römer I, Russ M, Vandebriel, RJ, Lynch I, Belinga-Desaunay, M, Valsami-Jones E, Connell SP, Smith IP, Duffin R, Boere JAF, Heusinkveld HJ, Albrecht C, de Jong WH, Cassee FR. The effect of zirconium doping of cerium dioxide nanoparticles 
on pulmonary and cardiovascular toxicity and biodistribution in mice after inhalation. Nanotoxicology. 2017 Aug;11(6):794-808. doi: 10.1080/17435390.2017.1357214.

Dekkers S, Oomen AG, Bleeker EA, Vandebriel RJ, Micheletti C, Cabellos J, Janer G, Fuentes $N$, Vázquez-Campos S, Borges T, Silva MJ, Prina-Mello A, Movia D, Nesslany F, Ribeiro AR, Leite PE, Groenewold M, Cassee FR, Sips AJ, Dijkzeul A, van Teunenbroek T, Wijnhoven SW. Towards a nanospecific approach for risk assessment. Regul Toxicol Pharmacol. 2016 May 30; 80:46-59. doi: 10.1016/j.yrtph.2016.05.037.

Vandebriel RJ, Dekkers S, de Jong WH, Cassee FR. An Update on NLRP3 Inflammasome Activation by Engineered Nanomaterials. Current Bionanotechnology, 2016; 2(1): 40-46. doi $10.2174 / 2213529402666160601122127$.

van Kesteren PC, Cubadda F, Bouwmeester H, van Eijkeren JC, Dekkers S, de Jong WH, Oomen AG. Novel insights into the risk assessment of the nanomaterial synthetic amorphous silica, additive E551, in food. Nanotoxicology. 2015 May; 9(4):442-52. doi: 10.3109/17435390.2014.940408.

Dekkers S, Bouwmeester H, Bos P, Peters R, Rietveld A, Oomen A. Knowledge gaps in risk assessment of nanosilica in food: evaluation of the dissolution and toxicity of different forms of silica. Nanotoxicology. 2013 Jun; 7(4):367-77. doi: 10.3109/17435390.2012.662250.

Dekkers S, Krystek P, Peters RJ, Lankveld DP, Bokkers BG, van Hoeven-Arentzen PH, Bouwmeester $\mathrm{H}$, Oomen AG. Presence and risks of nanosilica in food products. Nanotoxicology. 2011 Sep;5(3):393-405. doi: 10.3109/17435390.2010.519836.

Jeurissen SMF, Seyhan F, Kandhai MC, Dekkers S, Booij CJH, Bos PMJ, van der Fels-Klerx HJ. An indicator based 'traffic light' model to pro-actively assess the occurrence of mycotoxins in tree nuts. World Mycotoxin J. 2011; 494:405-12. doi.org/10.3920/WMJ2010.1278

van der Fels-Klerx HJ, Dekkers S, Kandhai MC, Jeurissen SMF, Booij CJH, de Heer C. Indicators for early identification of re-emerging mycotoxins. NJAS Wag J Life Sci 2010; 57(2):133-9. doi.org/10.1016/j.njas.2010.02.003

Wijnhoven SWP, Herberts C, Hagens WI, Oomen A, Heugens E, Roszek B, Bisschops J, Peijnenburg W, Gosens I, Van de Meent D, Dekkers S, De Heer C, Sips AJAM, De Jong W, Van Zijverden M, Geertsma R. Nano-silver, a review of available data and knowledge gaps in human and environmental risk assessment. Nanotoxicology, 2009 June; 3(2), 1-30 (2009). doi.org/10.1080/17435390902725914 
Miraglia M, Marvin HJ, Kleter GA, Battilani P, Brera C, Coni E, Cubadda F, Croci L, De Santis B, Dekkers S, Filippi L, Hutjes RW, Noordam MY, Pisante M, Piva G, Prandini A, Toti L, van den Born GJ, Vespermann A. Climate change and food safety: an emerging issue with special focus on Europe. Food Chem Toxicol. 2009 May;47(5):1009-21. doi: 10.1016/j.fct.2009.02.005.

Bouwmeester H, Dekkers S, Noordam MY, Hagens WI, Bulder AS, de Heer C, ten Voorde SE, Wijnhoven SW, Marvin HJ, Sips AJ. Review of health safety aspects of nanotechnologies in food production. Regul Toxicol Pharmacol. 2009 Feb; 53(1):52-62. doi: 10.1016/j. yrtph.2008.10.008.

Marvin HJ, Kleter GA, Prandini A, Dekkers S, Bolton DJ. Early identification systems for emerging foodborne hazards. Food Chem Toxicol. 2009 May;47(5):915-26. doi: 10.1016/j. fct.2007.12.021.

Freidig AP, Dekkers S, Verwei M, Zvinavashe E, Bessems JG, van de Sandt JJ. Development of a QSAR for worst case estimates of acute toxicity of chemically reactive compounds. Toxicol Lett. 2007 May 15; 170(3):214-22. doi: 10.1016/j.toxlet.2007.03.008.

Dekkers S, Telman J, Rennen MA, Appel MJ, de Heer C. Within-animal variation as an indication of the minimal magnitude of the critical effect size for continuous toxicological parameters applicable in the benchmark dose approach. Risk Anal. 2006 Aug; 26(4):867-80. doi: 10.1111/j.1539-6924.2006.00784.x.

Goldbohm RA, Tielemans EL, Heederik D, Rubingh CM, Dekkers S, Willems MI, Dinant Kroese E. Risk estimation for carcinogens based on epidemiological data: a structured approach, illustrated by an example on chromium. Regul Toxicol Pharmacol. 2006 Apr; 44(3):294-310. doi: 10.1016/j.yrtph.2006.01.007.

Dekkers S, de Heer C, Rennen MA. Critical effect sizes in toxicological risk assessment: a comprehensive and critical evaluation. Environ Toxicol Pharmacol. 2001 Jun;10(1-2):33-52. doi:10.1016/s1382-6689(01)00068-0. 


\section{Dankwoord}

Midden in de Corona lock-down kijk ik terug en realiseer ik me dat ik het heel erg getroffen heb met zoveel behulpzame, begripvolle en lieve begeleiders, collega's, vrienden en familieleden. Juist nu ik bijna alleen nog maar online contact met anderen heb, besef ik dat ik mijn waardering voor iedereen die aan de totstandkoming van dit proefschrift heeft bijgedragen veel te weinig heb laten blijken. Gelukkig heb ik vaak horen zeggen dat het dankwoord het meest gelezen hoofdstuk van menig proefschrift is, dus zie ik het als een kans om iedereen die mij bij deze klus geholpen en gesteund hebben hier alsnog (of nogmaals) te bedanken.

Allereerst wil ik natuurlijk mijn promotoren en co-promoteren bedanken. Flemming, bedankt voor de kans die jij mij gegeven hebt om te kunnen promoveren. Ik weet nog dat jij in de bus op weg naar een werkbezoek van de nanowerkgroep aan MESA+ vroeg of iemand misschien een geschikte kandidaat voor een PhD binnen het NANOMILE project wist. Toen ik eruit flapte dat mij dat zelf wel interessant leek, vroeg je gelijk of ik dat serieus meende en heb je me vervolgens geholpen om dit binnen mijn aanstelling bij het RIVM voor elkaar te krijgen. Bedankt ook voor je vertrouwen, steun, advies en kritische blik, maar vooral ook voor je onuitputtelijke optimisme en de vele introducties bij collega's binnen jouw netwerk. Jouw netwerk bleek voor mij een haast onuitputtelijke bron aan mogelijke samenwerkingen met veel verschillende instituten zowel binnen als buiten het NANOMILE consortium te zijn. Van het Nederlandse PamGene tot aan de Michigan State University. Helaas hebben niet al deze initiatieven ook tot wetenschappelijke publicaties geleid, maar dat lag vooral aan tijdgebrek vanuit mijn kant. Bert, bedankt voor je kritische en motiverende opmerkingen over de voortgang van mijn promotieonderzoek. Ondanks dat je inhoudelijk wat meer afstand tot dit onderzoek had, wist jij me in onze voortgangsgesprekken wel te motiveren om niet alleen aandacht en tijd aan de uitvoering, maar ook aan het schrijfwerk te besteden. Helaas, was deze motivatie alleen niet genoeg en bleek het in de praktijk toch lastig om het schrijven prioriteit te geven, waardoor dit langer duurde dan eigenlijk de bedoeling was. Rob en Wim bedankt voor jullie begeleiding. Omdat ik regelmatig drie tegenstrijdige adviezen van jullie en Flemming kreeg, heb ik heel veel van jullie geleerd en veel aan jullie kennis en expertise gehad. Daarnaast heb ik ook nog veel plezier met jullie beleefd tijdens de verschillende consortium meetings en andere internationale bijeenkomsten.

Daarnaast wil ik mijn collega's bij DMG bedanken. Daan, John en Paul bedankt voor het meedenken en uitvoeren van alle praktische, technische, analytische en logistieke uitdagingen in mijn eerste en gelijk ongekend ingewikkelde inhalatie studie met heel veel verschillende experimentele groepen, samples, tijdschema's, epjes en buizen. Jullie hebben me meerdere keren op de kast gekregen, maar daar zat ik graag om het overzicht te kunnen behouden. Harm, Ilse en Miriam, fijn dat ik jullie altijd even lastig kon vallen met een vraag 
of voor de nodige afleiding tijdens de gezellige koffiepauzes of het saaie stickeren (wat een stuk sneller gaat onder begeleiding van motiverende Spaanse ska). Ilse en Harm, wat fijn dat jullie mijn paranimfen willen zijn. Elise, Arjen, Angelique, Ingrid en Kelly bedankt voor de gezelligheid, het aanhoren van mijn frustraties en de toren-C-momentjes op vrijdag. Elmer, Geert, Rory, Jason, Jia en Jasper bedankt voor jullie inzet en bijdrage aan het onderzoek tijdens jullie stage. Dank ook aan de collega's van Intravacc voor hun bijdrage aan de uitvoering van de in vivo studies. Verder wil ik ook een aantal collega's bij GZB bedanken. Jolanda, Liset en Eric bedankt voor jullie hulp bij het in vitro werk en met name Jolanda voor je hulp bij het begeleiden van de stagiaires. Liset, Bhawani, Piet, Eric, Jolanda en Christina bedankt voor de analyse van de samples uit de in vivo studies en de endotoxine bepalingen. Bedankt ook Cees, Theo, Elske en Jan van VSP voor de mogelijkheid die jullie me hebben gegeven om dit onderzoek binnen mijn RIVM aanstelling te kunnen uitvoeren. En natuurlijk ook alle collega's van de nanowerkgroep en VSP voor hun interesse en lef om ook lang na de afronding van het NanoMILE project nog te durven vragen hoe het met mijn promotieonderzoek ging.

Many thanks (in English) to all international colleagues that contributed to this research within and outside the NanoMILE project. Mark, Roel (although you are not really an international colleague), Catrin, Tina and Julia for the great collaboration and for making optimal use of all samples from my first inhalation study. Isabella, Marie-France, Emily, Iseult and Eva for your advice, the distribution and the characterization of the NPs. Mike and Selina for your advice and the synthesis of the NPs. Lan, Jana and Robert for performing and sharing the data from previous and newly performed $\mathrm{CeO}_{2}$ inhalation studies. Tim, Jinkang, Albert, Shan and Mark for the omics analysis and for making it possible to include multiomics approaches within my thesis. Jim, Jack and colleagues for the great collaboration, for conducting the in vivo study on the adjuvant activity and for the warm welcome when I visited Michigan State University during the snowy cold weather. Leigh-Ann thank you for the great times we had together and for the opportunity to share the ups and downs in our research. And also thanks to all nano colleagues from other projects, conferences and meetings who showed their interest. Although I sometimes warned you not to ask again, I do appreciate your interest in the progress of my $\mathrm{PhD}$ work.

Naast alle lieve collega's op het werk, waren er natuurlijk ook vele vrienden en familieleden die mij gesteund hebben. Soms door mij wat vaker te moeten missen "omdat ik weer zo nodig aan mijn boekje moest werken" en soms juist door mij daar even van weg te trekken voor de nodige ontspanning of sportieve inspanning. Voor al deze mensen en voor iedereen die ik nog niet genoemd heb, bedankt voor jullie steun, begrip en geduld.

Myra en Eldin, bedankt voor de tijd die jullie mij hebben gegeven. Volgens mij bedank ik jullie nog het meeste door hier nu straks ook echt letterlijk de laatste punt achter te zetten. 
Want ja, als dit boekje eindelijk af is, heb ik inderdaad meer tijd voor jullie en zal ik me in de weekenden niet meer hele dagen terugtrekken achter mijn computer.

Mark, de belangrijkste heb ik inderdaad tot het laatste bewaard. Zonder jou (en mijn twee ogen) was dit me niet gelukt. Bedankt voor je steun, geduld, vertrouwen, opbeurde woorden en vooral ook voor de ruimte die je me gegeven hebt om dit te kunnen doen. Maar het meeste nog bedankt voor wie jij bent en dat je er ook voor mij bent. 

